\title{
Biophysical and Crystallographic Characterization of Spliceosomal DExD/H-box ATPases
}

\author{
Dissertation \\ for the award of the degree \\ "Doctor rerum naturalium” \\ of the Georg-August-Universität Göttingen
}

\begin{abstract}
within the doctoral program
"Biomolecules: Structure - Function - Dynamics"

of the Georg-August-University School of Science (GAUSS)
\end{abstract}

submitted by

\section{Florian Hamann}

from Detmold

Göttingen, 2019 

Members of the Thesis Advisory Committee

\section{Prof. Dr. Ralf Ficner (Reviewer 1)}

Georg-August-University Göttingen

Institute for Microbiology and Genetics

Department for Molecular Structural Biology

Prof. Dr. Reinhard Lührmann (Reviewer 2)

Max-Planck-Institute for Biophysical Chemistry

Department of Cellular Biochemistry

\section{Prof. Dr. Holger Stark}

Max-Planck-Institute for Biophysical Chemistry

Department of Structural Dynamics

Members of the Examination Board

Dr. Sarah Adio

Georg-August-University Göttingen

Institute for Microbiology and Genetics

Department for Molecular Structural Biology

\section{Prof. Dr. Kai Tittmann}

Georg-August-University Göttingen

Department of Molecular Enzymology

\section{Prof. Dr. Markus Bohnsack}

University Medical Center Göttingen

Department of Molecular Biology 

Hereby I declare that I prepared the doctoral thesis in hand titled "Biophysical and Crystallographic Characterization of Spliceosomal DExD/H-box ATPases" independently and with no sources and aids other than quoted. The thesis has not been submitted elsewhere.

Florian Hamann, June 2019 



\section{Contents}

Chapter 1: Introduction.....................................................................................................................1

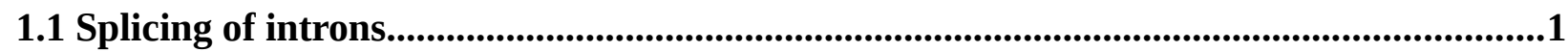

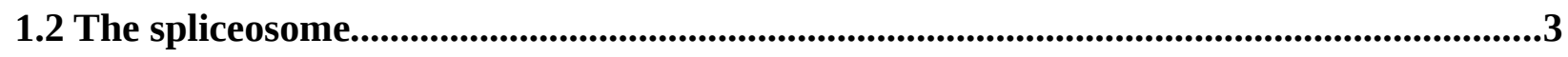

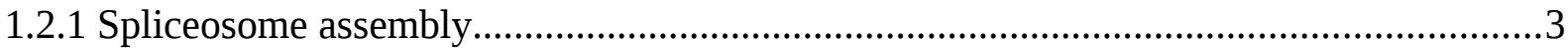

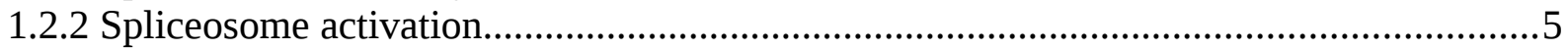

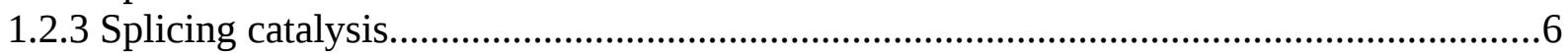

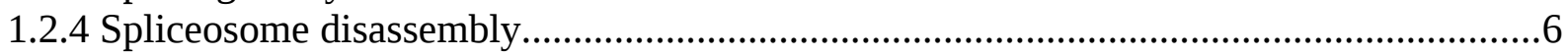

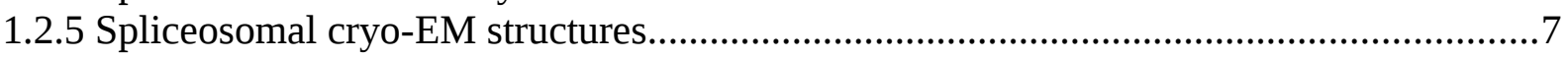

1.3 Key players of the spliceosome...........................................................................................8

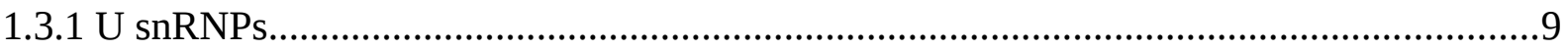

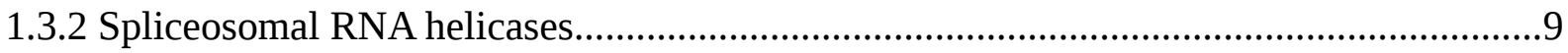

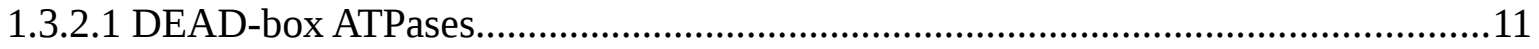

1.3.2.2 Ski2-like helicase Brr2....................................................................................... 12

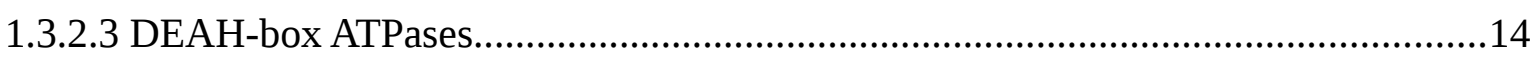

1.4 DEAH-box ATPases examined in this thesis...........................................................16

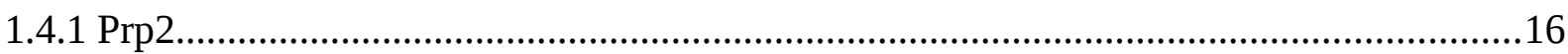

1.4.1.1 Interplay of Prp2 with its G-patch protein Spp2 ................................................17

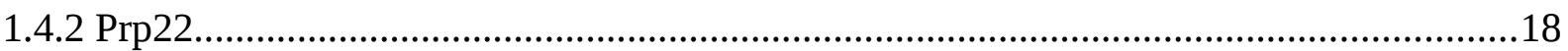

1.5 Scope - Insights into the translocation mechanism of DEAH-box ATPases.....................20

Chapter 2: Crystal structure of the spliceosomal DEAH-box ATPase Prp2.................21

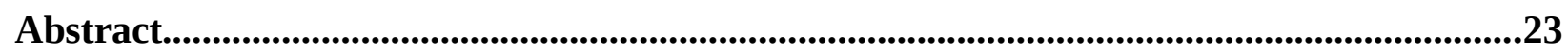

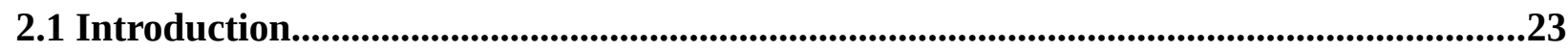

2.2 Materials and methods..................................................................................................24

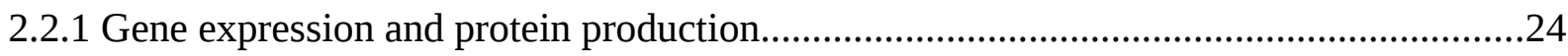

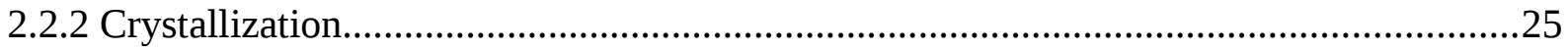

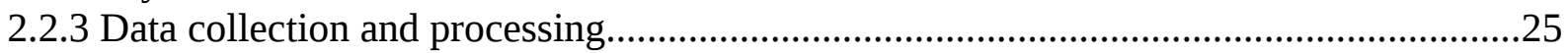

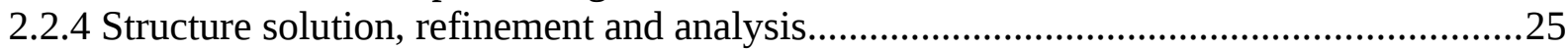

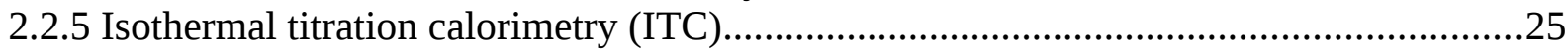

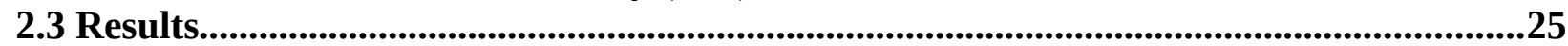

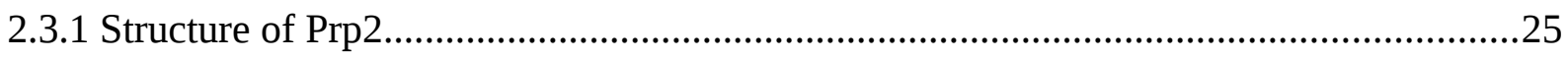

2.3.2 Structural flexibility of the C-terminal domains.......................................................26

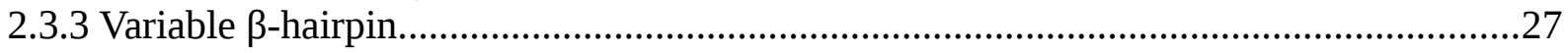

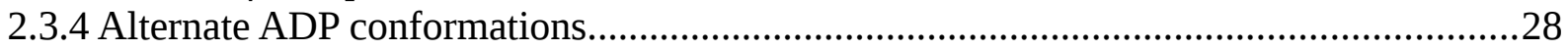

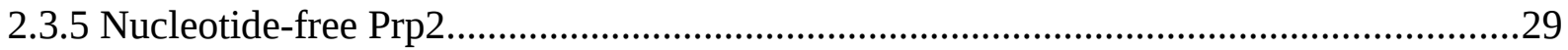

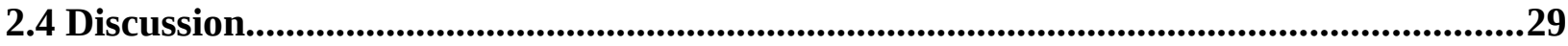

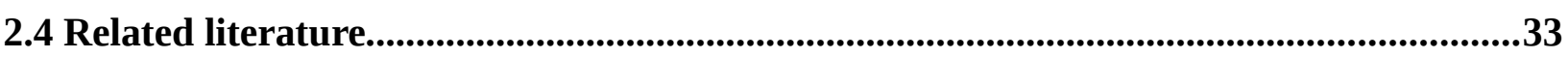

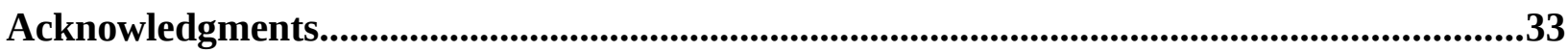

Funding information.......................................................................................................................................33

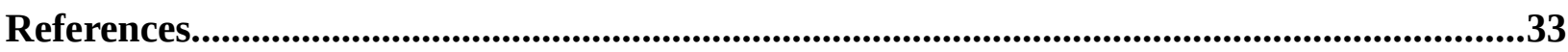

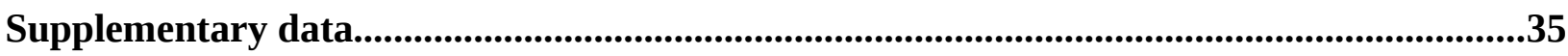


Chapter 3: Structural analysis of the interaction of Spp2 G-patch with Prp2...............44

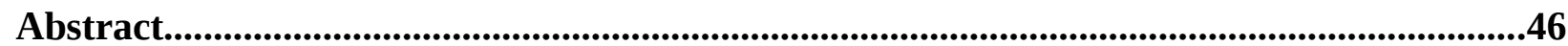

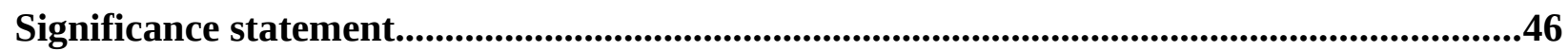

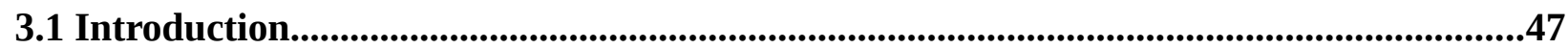

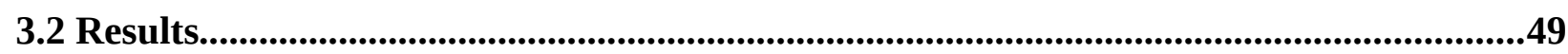

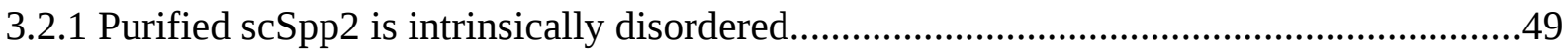

3.2.2 Crystal structure of the ctPrp2-ctSpp2 ${ }_{\text {G-patch }}$ complex......................................................49

3.2.3 Cross-linking data confirm Spp2 binding site in solution...........................................51

3.2.4 The G-patch of Spp2 populates transient helical conformations in solution...................52

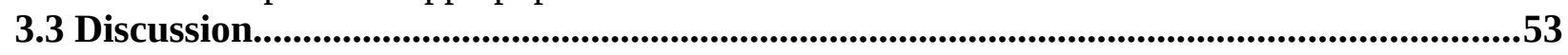

3.4 Materials and methods..............................................................................................57

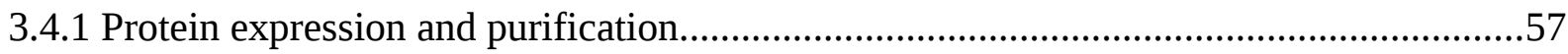

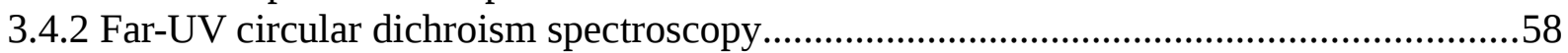

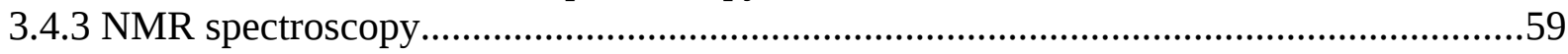

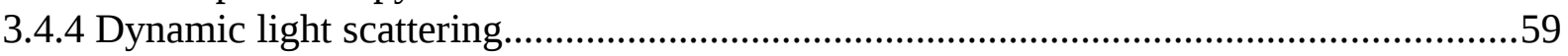

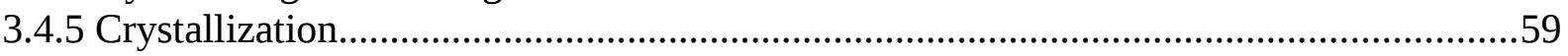

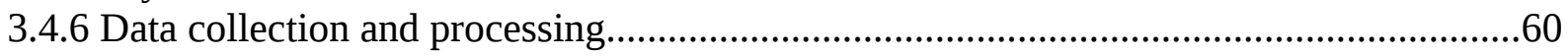

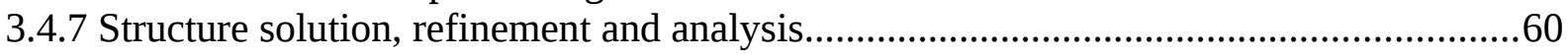

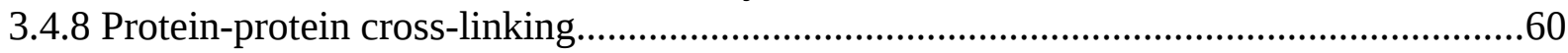

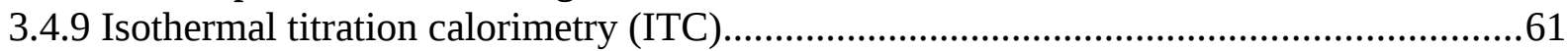

Acknowledgments..................................................................................................................................61

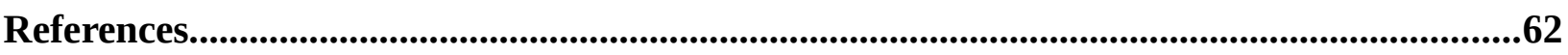

Supplementary Information................................................................................................72

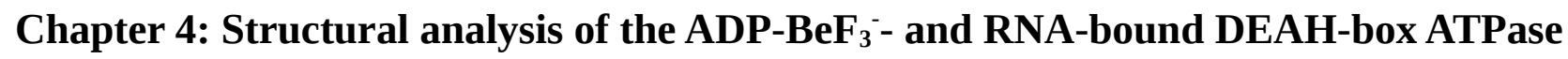
Prp2..............................................................................................................84

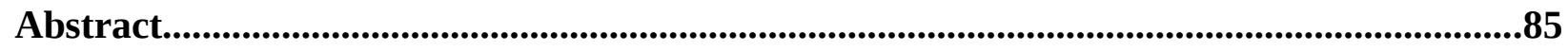

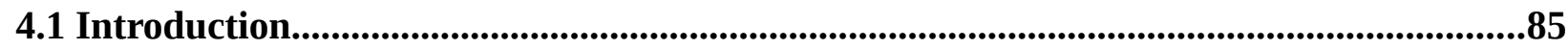

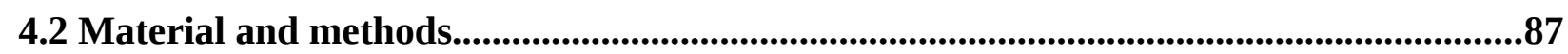

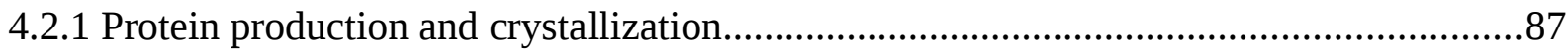

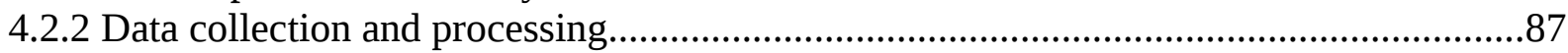

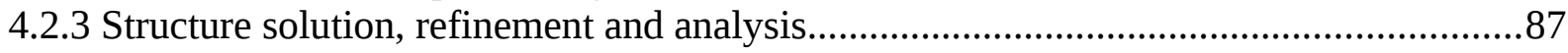

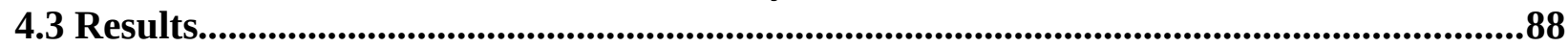

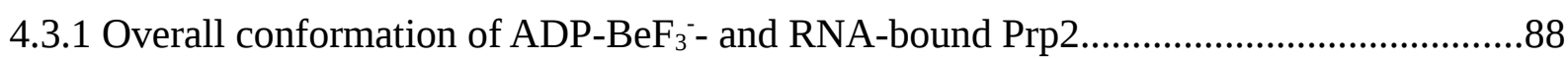

4.3.2 Putative exit channel for phosphate after ATP hydrolysis...........................................89

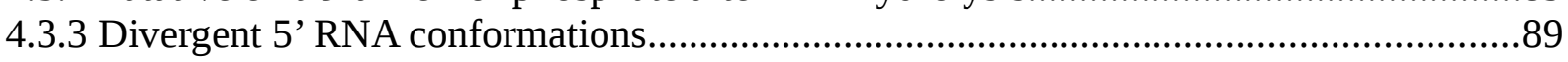

4.3.4 Loop in C-terminal domain threads 5' RNA region through tunnel in the ATP-bound

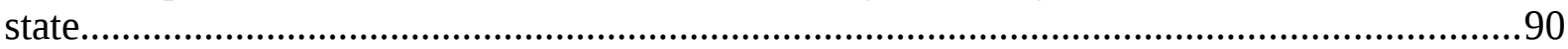

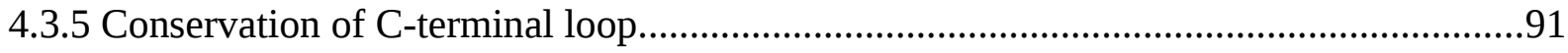

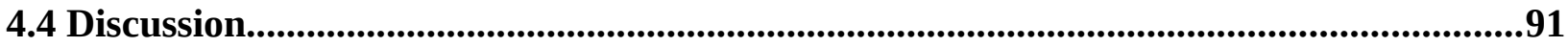

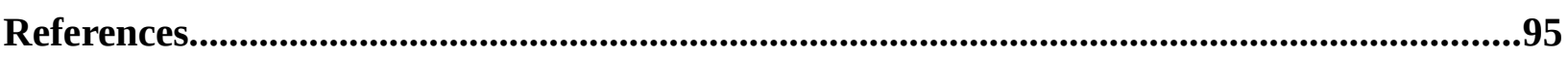

Supplementary information........................................................................................................................105

Chapter 5: Structural basis for RNA translocation by DEAH-box ATPases...............107

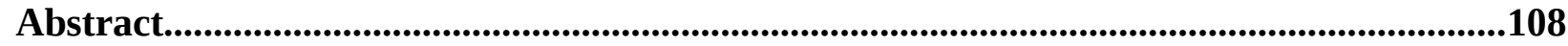

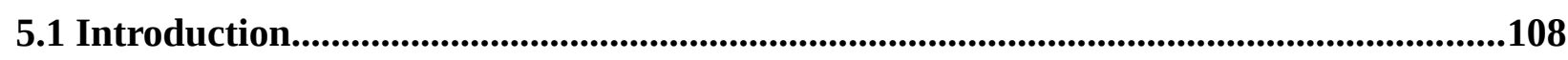




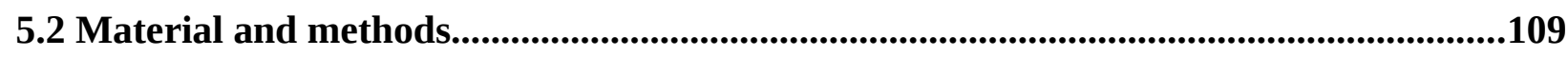

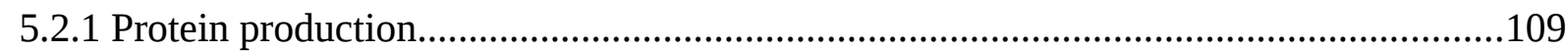

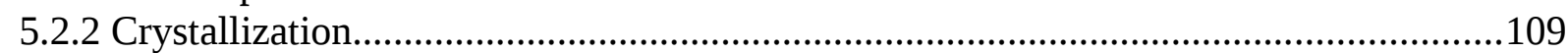

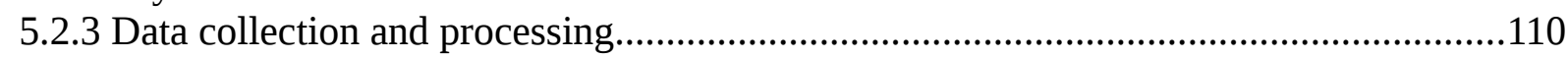

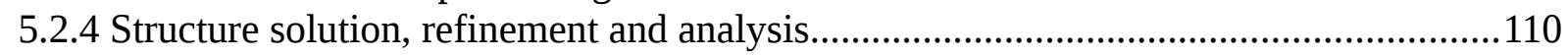

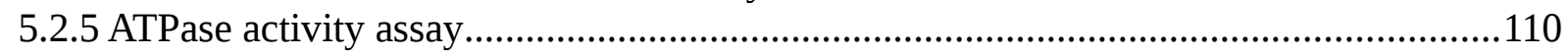

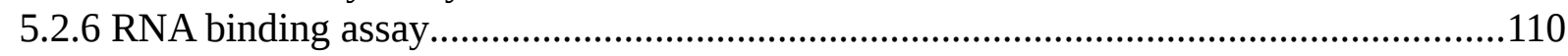

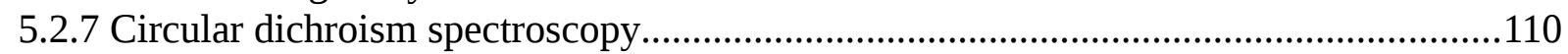

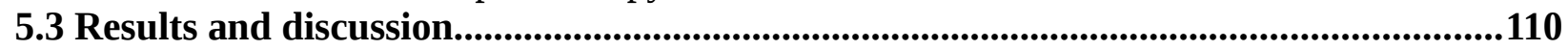

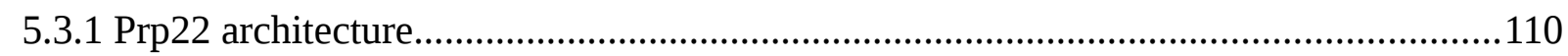

5.3.2 RNA binding of Prp22...............................................................................................111

5.3.3 Intrinsic mobility of RecA2 domain enables open conformation of helicase core........114

5.3.4 DEAH-box ATPases translocate at a step-size of one RNA nucleotide per hydrolyzed

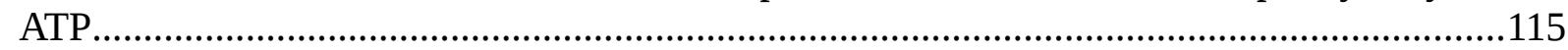

5.3.5 Helicase core rearrangements are driven by a serine in motif V that senses catalytic state

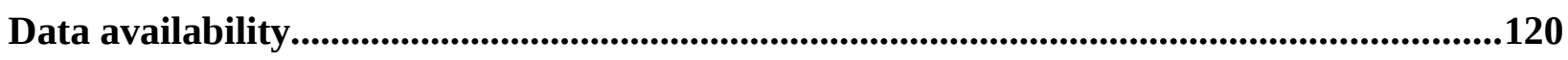

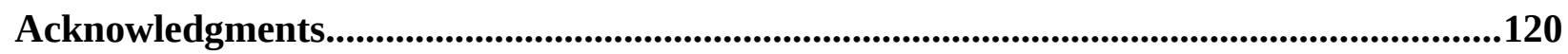

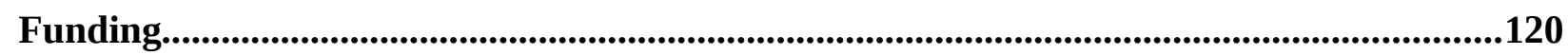

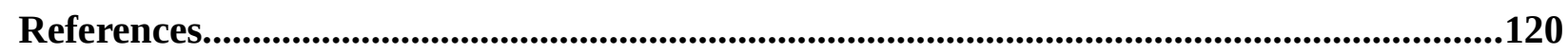

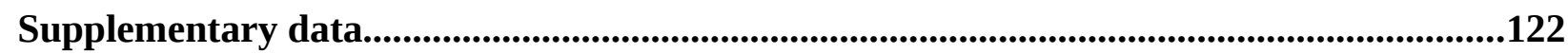

Chapter 6: Discussion and Outlook........................................................................138

6.1 Conservation of translocation in SF1 and SF2 members..............................................138

6.2 Conservation of catalytic state sensing and RecA2 positioning.....................................141

6.3 DEAH-box ATPase domain dynamics - revisited.......................................................144

6.4 Translocation of DEAH-box ATPases in the spliceosome...........................................147

6.5 Insights into the Prp43-Ntr1 interaction in the spliceosome...........................................150

Chapter 7: Abstract..............................................................................................................................153

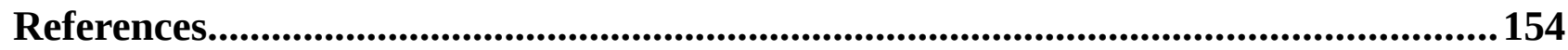

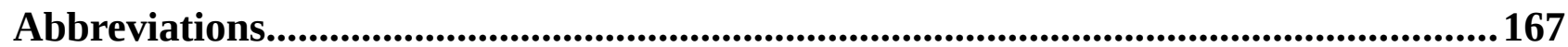

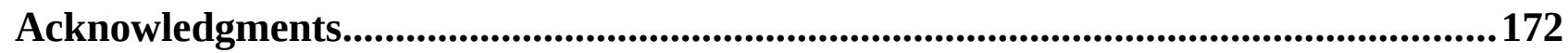

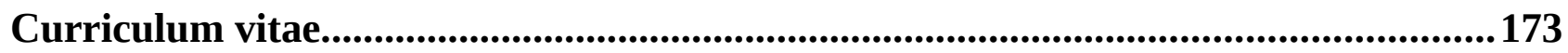




\section{Chapter 1: Introduction}

The survival of living organisms depends on the maintenance of homeostasis and the response to countless external stimuli. Undoubtedly, proteins play a vital role in all of these processes due to their great functional versatility and they are responsible for cellular roles like catalysis, transport and scaffolding as well as serving as storage molecules. To ensure all these functions, the cell requires the constant production of these macromolecules. Therefore, the information for their synthesis is stored in the genes and accessible when needed.

It has been postulated that the production of proteins follows a strict directional transfer of information and has been denominated the central dogma of molecular biology (Crick, 1970). Here, the genes encoded in the DNA (deoxyribonucleic acid) serve as a library for the proteins to be synthesized that are first transcribed into RNA (ribonucleic acid) and then finally translated into a polypeptide chain. While there are special cases as reverse transcription and RNA replication they display exceptions mainly known from viruses (Hu and Hughes, 2012; Lohmann, 2013). Basically, the central dogma of molecular biology implies that one gene ultimately results in one protein product and that all proteins are already completely predefined by the sequence of the bases on the DNA level. But cells have found ways to circumvent this limitation and expand their genetic information. While mechanisms like proteolytic cleavage and post-translational modification impact structure and function of proteins, these modifications occur only after protein synthesis and do not contradict Crick's central dogma. In contrast, a process called splicing already interferes in the transfer of the genetic information between transcription and translation by altering the RNA and thereby expanding the possible outcome of protein products.

\subsection{Splicing of introns}

Once the gene-encoding DNA is transcribed into pre-mRNA (precursor messenger RNA) in the nucleus it has to undergo various post-transcriptional modifications before it can be exported as a mature mRNA out of the nucleus for translation. On the one hand, a $\mathrm{m}^{7} \mathrm{G}$ (7-methylguanosine) cap is coupled to the 5' end of the pre-mRNA and a poly(A)-tail is added to the 3' end, which mainly contribute to the stability of the mRNA and/or serve as signal for the nuclear export (Drummond et al., 1985). On the other hand, the pre-mRNA does not only contain coding sequences called exons, but also non-coding introns, that need to be removed prior to translation. The excision of introns is termed splicing and there are two different types of splicing. While it is possible that only all present introns are cleaved for the final product, the mechanism of alternative splicing can also result in the excision of exons. Mechanisms like 5'/3' splice site selection or 


\section{Chapter 1: Introduction}

cassette exon skipping recognize flanking intronic regions that influence the cleavage of a specific exon or an exon can be treated as an intron regardless of the presence of surrounding introns (Nilsen and Graveley, 2010). This dramatically increases the possible outcome of one transcript and expands the genetic information stored in the genes.

Introns can be divided into four classes: tRNA (transfer RNA), group I, group II and spliceosomal introns. The removal of tRNA introns underlies different mechanisms in all domains of life. tRNA introns of archea and eukaryotes require a mechanism involving an endonuclease, a ligase and a phosphotransferase for their excision, whereas bacterial tRNA introns belong to a group of self-splicing introns (Abelson et al., 1998). Group I and II introns share the common property to be self-splicing introns that do not require any additional energy for their cleavage (Kruger et al., 1982). While both groups perform the excision of the intron via two subsequent transesterification reactions following an $\mathrm{S}_{\mathrm{N}} 2$ nucleophilic substitution mechanism, they show significant differences. Group I introns require a guanosine co-factor that attacks the phosphodiester bond of the 5'SS (5' splice site) with its 3'OH (3' hydroxy group) leading to the first cleavage reaction and the attachment of the co-factor to the 5' end of the intron (Fig. 1a) (Cech, 1990). The 3'OH of the cleaved 5' exon is now able to perform a nucleophilic attack on the phosphodiester bond of the 3'SS (3' splice site) resulting in the ligation of both exons and a removed linear intron. In contrast to group I introns, group II introns do not require an additional co-factor. In this group of introns a conserved adenosine

\section{a}
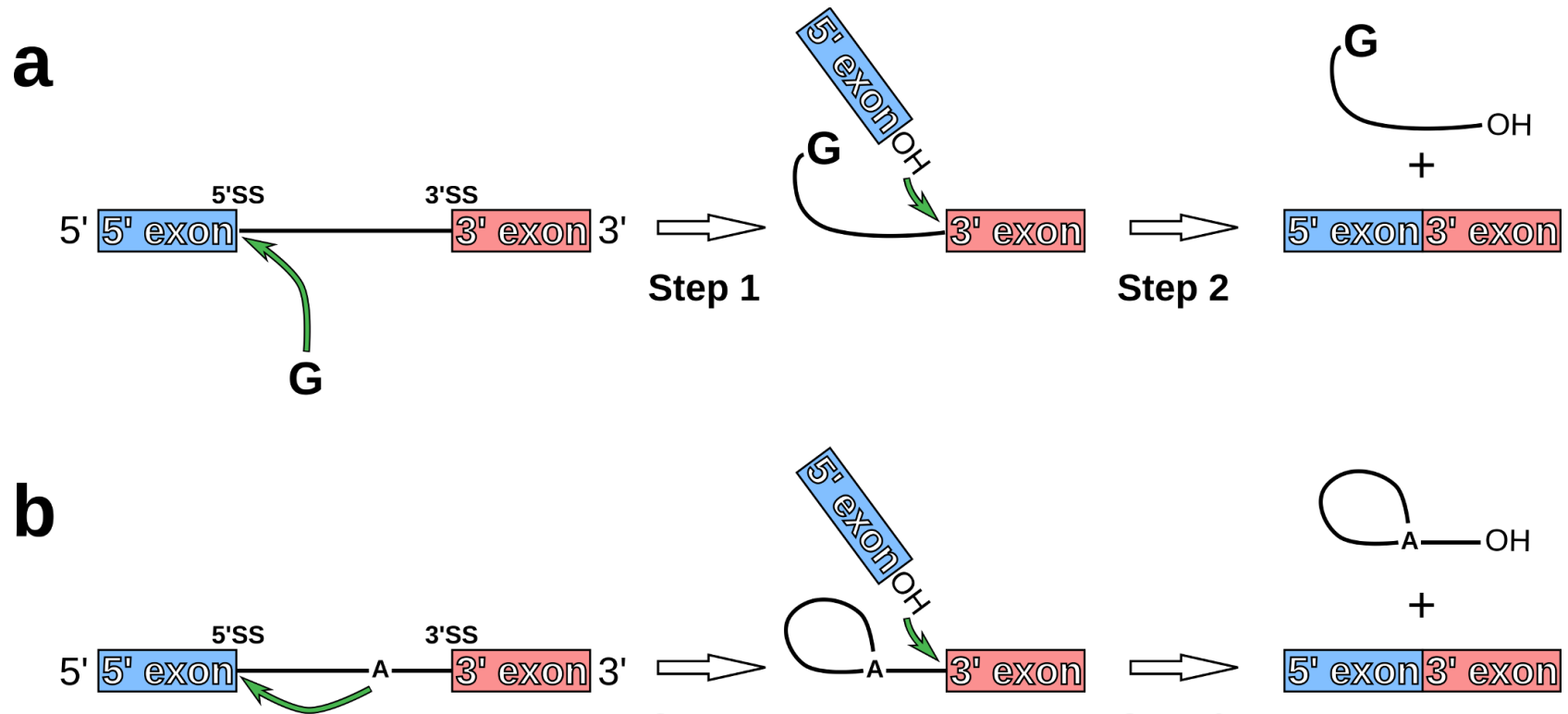

\section{Step 1}

Step 2

Figure 1: Splicing of group I and II introns. Group I and II introns are composed of a 5'SS and a 3'SS and are sandwiched between two exons. In both groups the excision of the introns are performed via two transesterification steps. (a) Group I introns require a guanosine co-factor for the first nucleophilic attack on the 5'SS. After the second nucleophilic attack performed by the 3'OH of the 5' exon on the 3'SS, the exons are ligated and a cleaved linear intron is obtained. (b) Group II intron do not need a co-factor and instead the first reaction is performed by a conserved adenosine of the intron. The second nucleophilic attack also performed by the 3'SS of the 5' exon on the 3'SS leads to ligation of the exons and excision of the intron-lariat. Nucleophilic attacks are highlighted as green arrows. This figure was adopted from Will and Lührmann (2011). 


\section{Chapter 1: Introduction}

in the intron sequence takes over the role of the co-factor and facilitates the first transesterification reaction via a nucleophilic attack with its 2'OH (Fig. 1b). The second transesterification reaction is performed by an attack of the 3'OH group at the 3' end of the 5' exon with the phosphodiester bond of the 3'SS. This yields two ligated exons and an excised the so-called intron-lariat (Peebles et al., 1986).

The vast majority of introns however are removed with the help of the spliceosome, a highly dynamic molecular machine with the purpose of aiding the removal of introns in eukaryotes (Will and Lührmann, 2011). Spliceosomal introns consist as well of a 5'SS, 3'SS and a conserved adenosine as part of the branchsite, which is located 18 to 40 nucleotides upstream of the 3'SS. Although the basic removal mechanism of spliceosomal introns is highly similar to the one from group II introns resulting in the ligation of two exons and the formation of an intron-lariat (Fig. 1b), spliceosomal introns lack the ability of self-splicing and are strictly dependent on the involvement of the spliceosome.

\subsection{The spliceosome}

The spliceosome is a multimegadalton complex composed of RNA elements as part of the U-snRNPs (uridine-rich small nuclear ribonucleic particles) and a large number of protein components, that are involved in an intricate and highly dynamic interplay with the final purpose of mediating the intron excision out of pre-mRNAs (Wahl et al., 2009). The complex has no predefined active site and thus has to be built up until reaching a state competent of performing the two transesterification steps. This implicates constant conformational and compositional rearrangements of the spliceosome. Almost none of the spliceosomal components is present throughout all stages of a splicing cycle and every recruitment or displacement dictates the next step in the splicing process. Since the spliceosome is a single-turnover molecular machine, for each intron to be spliced the complex has to be assembled first and only upon activation it is able to perform the catalytic steps (Fig. 2). Finally, the complex is completely disassembled and every single component is available for another round of splicing.

\subsubsection{Spliceosome assembly}

The binding of the U1 snRNP to the 5'SS initiates the spliceosomal assembly on the intronic region of the pre-mRNA. Here, the 5' end of the U1 snRNA localizes the 5'SS via base-pairing (Séraphin et al., 1988; Siliciano and Guthrie, 1988; Zhuang and Weiner, 1986). Together with the recognition of the branch-site by the two proteins Msl5 (Mus Synthetic-lethal 5) and Mud2 (Mutant U1 Die 2), the complex E is formed, which does not require the consumption of ATP (adenosine triphosphate) for its assembly (Abovich and 


\section{Chapter 1: Introduction}

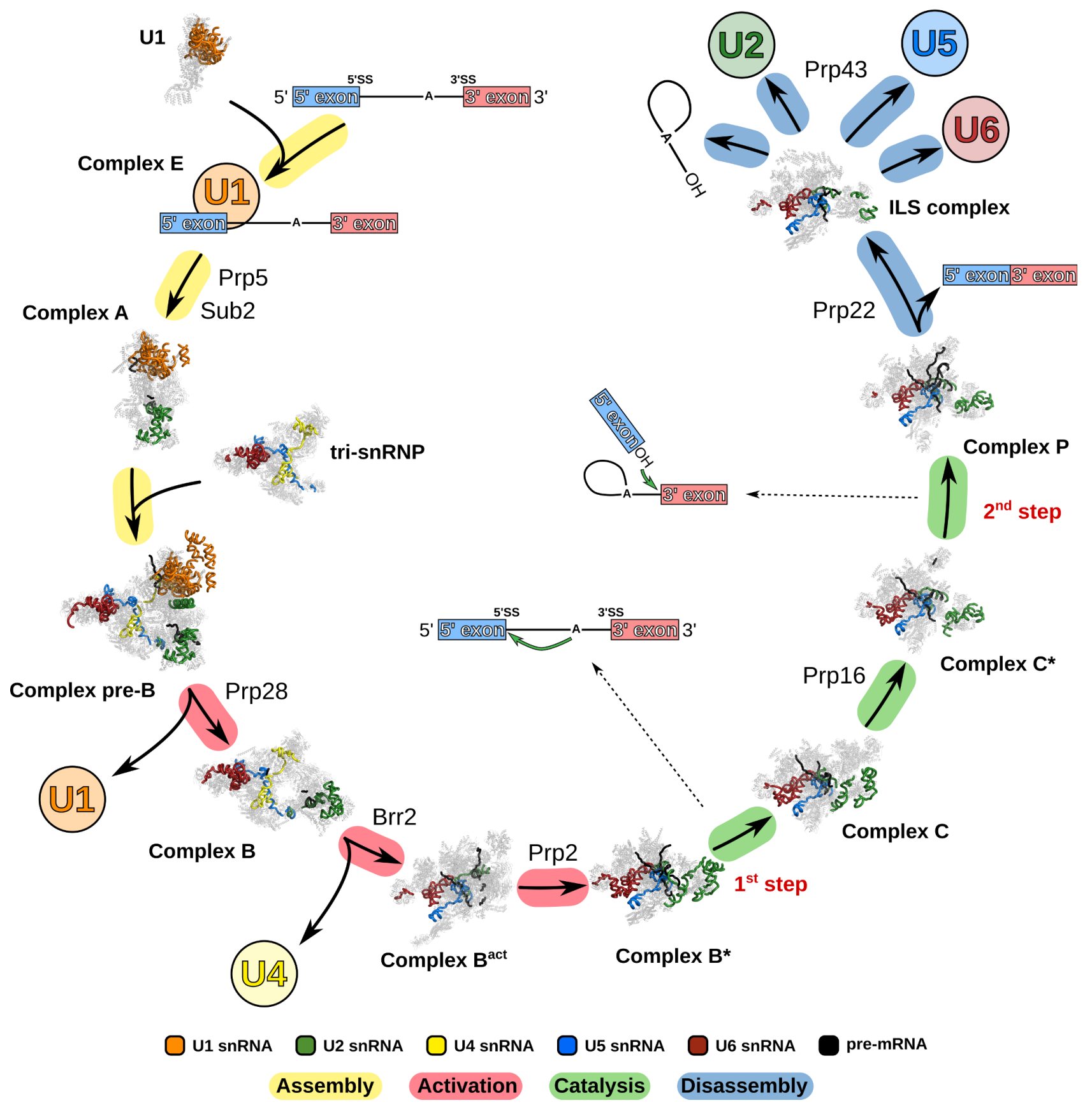

Figure 2: The splicing cycle. The spliceosome is a highly dynamic complex that is composed of RNA (U-snRNAs) and protein components. It is assembled in a sequential manner until a catalytically active complex is obtained that is able to perform the two transesterification steps. Once the intron is cleaved the spliceosomal complex is completely disassembled and all components are ready for a new round of splicing. Spliceosomal structures from S. cerevisiae of each splicing step, if available, are shown as cartoon models. The U-snRNAs are highlighted in different colors, whereas the protein components are displayed as gray semi-transparent cartoons. Complexes not structurally characterized are shown in a schematic representation. The eight conserved ATP-dependent RNA helicases crucial for the transition of several spliceosomal complexes are labeled at the corresponding step. This figure was inspired by Galej (2018). The following PDB depositions were used for the shown complexes: U1 - 5uz5; complex A - 6g90; tri-snRNP - 5gan; complex pre-B - 5zwn - 5zwm; complex B - 5nrl; complex Bact - 5gm6; complex B* - 6j6g; complex C 5lj5; complex C* - 5mq0; complex P - 6bk8; ILS complex - 5y88. 


\section{Chapter 1: Introduction}

Rosbash, 1997; Berglund et al., 1997). The subsequent formation of complex A represents the first ATPdependent step and is achieved by the involvement of two DEAD-box helicases, Prp5 (pre-mRNAprocessing factor 5) and Sub2 (Suppressor of BRR1 protein 2). While Sub2 has been suggested to mediate the displacement of the Msl5:Mud2 dimer thereby making the branch-site available for base-pairing with the newly recruited U2 snRNP, Prp5 probably remodels the U2 snRNA to influence its binding to the branch-site (Kistler and Guthrie, 2001; Xu and Query, 2007). The recruitment of the U4/U6.U5 tri-snRNP to the complex A leads to the formation of the complex pre-B, which is the only spliceosomal complex that contains all five U snRNPs (Bai et al., 2018; Boesler et al., 2016). The tri-snRNP is only loosely bound to this new complex and its conformation remains similar to the structure of its free particle (Nguyen et al., 2016). It is in the following activation steps that these components get more integrated, ultimately leading to the formation of an active site competent of performing the catalytic reactions.

\subsubsection{Spliceosome activation}

While the complex pre-B contains almost all necessary components known to be important for catalysis, the active site has first to be formed which implies major rearrangements in terms of structure and composition. The generation of complex B is achieved by the ATP-dependent action of the DEAD-box helicase Prp28, which promotes the exchange of the U1 snRNP for the U6 snRNP at the 5'SS (Chen et al., 2001; Staley and Guthrie, 1999). This eventually leads to the release of the U1 snRNP. The unwinding of the U4/U6 duplex mediated by the Ski2-like (Superkiller protein 2-like) helicase Brr2 (Bad response to refrigeration 2) ensures the transition to the activated complex Bact (Laggerbauer et al., 1998; Raghunathan and Guthrie, 1998a). The disruption of this duplex does not only allow the U6 snRNA to form the ISL (internal stem-loop) and basepairing with the U2 snRNA, but also triggers the release of the U4 snRNP (Madhani and Guthrie, 1992). During this event numerous other splicing factors are displaced (Snu66, Prp6, LSm and Dib1), but also many others are recruited (NTC, NTR and RES complexes; Cwc24 and Cwc27) (Fabrizio et al., 2009). The DEAH-box ATPase Prp2 finally succeeds to catalytically activate the spliceosome, leading to the formation of the catalytically activated B* complex (Kim and Lin, 1996). The involvement of Prp2 results in the displacement of SF3a (splicing factors 3a) and SF3b (splicing factors 3b) complexes together with Cwc24 (Complex with CEF1 protein 24) and Cwc27, which has been suggested to increase the accessibility of the branch-site adenosine in preparation for the first nucleophilic attack (Lardelli et al., 2010; Teigelkamp et al., 1994; Warkocki et al., 2009). To fulfill its task, Prp2 is strictly dependent on the interaction with the G-patch (glycine-rich patch) protein Spp2 (Suppressor of PRP protein 2) (Roy et al., 1995; Warkocki et al., 2015). 


\section{Chapter 1: Introduction}

\subsubsection{Splicing catalysis}

The involvement of Prp2 also creates a high affinity binding site for Yju2 and Cwc25, both of which play a critical role in the positioning of the U2, U5 and U6 snRNAs as well as the pre-mRNA in the B* complex (Chiu et al., 2009; Liu et al., 2007). The obtained arrangement of the RNA components facilitates the first transesterification reaction, also called branching, obtaining complex C with a cleaved 5' exon and an intronlariat formation still attached to the 3' exon (Galej et al., 2016; Krishnan et al., 2013). In order for the second transesterification step to happen, the spliceosome still needs to undergo important remodeling, which is mediated by the DEAH-box ATPase Prp16 (Konarska et al., 2006; Ohrt et al., 2013; Schwer and Guthrie, 1991, 1992; Smith et al., 2008). Prp16 is located at the 3'SS region and it has been proposed that its ability to translocate applies pulling forces on the pre-mRNA that for example contribute to the fidelity of the branchsite recognition and the release of splicing factors Cwc25 and Yju2 (Burgess and Guthrie, 1993; Galej et al., 2016; Semlow et al., 2016; Tseng et al., 2011). The involvement of Prp16 guarantees the formation of complex $C^{*}$, which is now able to perform the second transesterification step. In this complex the active site is now accessible due to a repositioning of the Prp8 RNaseH-like domain aided by the involvement of Prp18 and Slu7 (Synthetic letahl with U2 snRNA protein 7) (Bertram et al., 2017a; Fica et al., 2017; James et al., 2002; Ohrt et al., 2013; Yan et al., 2017). The second transesterification reaction results in complex P, where both exons are found ligated (Bai et al., 2017; Liu et al., 2017; Wilkinson et al., 2017).

\subsubsection{Spliceosome disassembly}

Once the spliceosome has achieved its final goal, the excision of the intron, the complete disassembly of the complex can be initiated. Similar to Prp2 and Prp16, it has been suggested that Prp22 acts as a translocase that induces conformational rearrangements from a distance by pulling on the mRNA downstream from the splice junction (Aronova et al., 2007; Company et al., 1991; Fica et al., 2017; Mayas et al., 2006; Schwer, 2008; Semlow et al., 2016). This mechanism is thought to disrupt base-pairings of the mRNA with the U5 snRNA and thereby facilitate the release of the mature mRNA. With the release of the mRNA, the ILS (intron-lariat spliceosome) complex is obtained (Wan et al., 2017). The DEAH-box ATPase Prp43 is responsible the final disassembly of the remaining U2, U5 and U6 snRNPs as well as of the intron-lariat (Arenas and Abelson, 1997; Martin et al., 2002). For its function it relies on the interaction with the G-patch protein Ntr1 (Nineteen complex-related protein 1) as it is not only involved in the catalytic stimulation of Prp43 but also in its regulation (Fourmann et al., 2016; Tauchert et al., 2017; Tsai et al., 2007). After accomplished disassembly, spliceosomal proteins as well as snRNPs are recycled and made available for new rounds of splicing (Raghunathan and Guthrie, 1998b). 


\section{Chapter 1: Introduction}

\subsubsection{Spliceosomal cryo-EM structures}

The described dynamics of the spliceosome have been studied extensively using genetic and biochemical approaches over the last decades, but it is the impressive technical development of cryo-EM in recent years that has boosted the structural knowledge of the spliceosome at an unprecedented pace. The determination of the tri-snRNP and ILS complex in 2015 initiated the structural elucidation of spliceosomal complexes and up to date all major steps are structurally characterized with the exception of complex E (Fig. 2, Table 1). The information gained by these structures have been used to answer longstanding questions that previous studies have not been able to definitively tackle. For instance, it could be demonstrated that spliceosomal introns and group II self-splicing introns share a common ancestor and that the catalysis for intron cleavage is RNAbased (Jacquier, 1990). The structural similarity of the active site in an early determined ILS complex compared with the structure of a post-catalytic group II intron provided the evidence that the spliceosome activity is based on ribozyme activity (Robart et al., 2014; Yan et al., 2015). Further on, it could be proven on a structural manner that two catalytic metal ions are involved in the two transesterification steps (Fica $e t$ al., 2013, 2017; Steitz and Steitz, 1993; Yan et al., 2016). These two reactions are performed by a single active site that requires extensive conformational remodeling not only to be formed, but also to guarantee the subsequent execution of the two transesterification steps. These structural changes of the spliceosome could only be deduced by previous genetic and biochemical approaches, but can be more precisely envisioned with the help of the repertory of different structurally characterized spliceosomal stages.

Table 1: Published cryo-EM structures of spliceosomal complexes.

\begin{tabular}{|c|c|c|c|c|c|}
\hline $\begin{array}{l}\text { Spliceosomal } \\
\text { complex }\end{array}$ & Species & PDBid(s) & $\begin{array}{c}\text { Overall } \\
\text { resolution }(\AA)\end{array}$ & References & RNA helicase in complex \\
\hline U1 snRNP & S. cerevisiae & 5uz5 & 3.7 & Li et al., 2017 & - \\
\hline \multirow[t]{4}{*}{ tri-snRNP } & S. cerevisiae & $\begin{array}{l}\text { 5gan, 5gap, } \\
\text { 5gao, 5gam }\end{array}$ & 5.9 / 3.7 & $\begin{array}{l}\text { Nguyen et al., 2015, } \\
2016\end{array}$ & Brr2 \\
\hline & & 3jcm & 3.8 & Wan et al., 2016a & Brr2 \\
\hline & H. sapiens & 3jcr & 7.0 & Agafonov et al., 2016 & Brr2, Prp28 \\
\hline & & 6qw6 & 2.9 & Charenton et al., 2019 & Brr2, Prp28 \\
\hline A & S. cerevisiae & 6g90 & 4.0 & Plaschka et al., 2018 & - \\
\hline \multirow[t]{3}{*}{ pre-B } & S. cerevisiae & 5zwm, 5zwn & $3.4-4.6$ & Bai et al., 2018 & Brr2, Prp28 \\
\hline & H. sapiens & 6ah0 & 5.7 & Zhan et al., 2018a & Brr2, Prp28 \\
\hline & & $6 q x 9$ & 3.3 & Charenton et al., 2019 & Brr2, Prp28 \\
\hline \multirow[t]{4}{*}{ B } & S. cerevisiae & $5 n r l$ & $3.6-17.2$ & Plaschka et al., 2017 & Brr2 \\
\hline & & 5zwo & 3.9 & Bai et al., 2018 & Brr2 \\
\hline & H. sapiens & $509 z$ & 4.5 & Bertram et al., 2017b & Brr2 \\
\hline & & 6ahd & 3.8 & Zhan et al., 2018a & Brr2 \\
\hline $\mathrm{B}^{\text {act }}$ & S. cerevisiae & $5 g m 6$ & 3.5 & Yan et al., 2016 & Brr2, Prp2 \\
\hline
\end{tabular}




\section{Chapter 1: Introduction}

\begin{tabular}{|c|c|c|c|c|c|}
\hline & & 5lqw & 5.8 & Rauhut et al., 2016 & Brr2, Prp2 \\
\hline & H. sapiens & $6 \mathrm{ff} 4,6 \mathrm{ff} 7$ & 3.4 & Haselbach et al., 2018 & Brr2, Prp2 \\
\hline & & $\begin{array}{l}5 z 56,5 z 57 \\
5 z 58\end{array}$ & $4.9-6.5$ & Zhang et al., 2018 & Brr2, Prp2 \\
\hline $\mathrm{B}^{*}$ & S. cerevisiae & $\begin{array}{l}6 j 6 g, 6 j 6 h, \\
6 j 6 n, 6 j 6 q\end{array}$ & $2.9-3.8$ & Wan et al., 2019 & - \\
\hline \multirow[t]{3}{*}{$\mathrm{C}$} & S. cerevisiae & $5 g m k$ & 3.4 & Wan et al., 2016b & - \\
\hline & & 5lj3, 5lj5 & 3.8 & Galej et al., 2016 & Brr2, Prp16 \\
\hline & H. sapiens & $5 y z g$ & 4.1 & Zhan et al., 2018b & $\begin{array}{l}\text { Brr2, Prp16, eIF4A-III, } \\
\text { Aquarius }\end{array}$ \\
\hline \multirow[t]{4}{*}{$\mathrm{C}^{*}$} & S. cerevisiae & 5wsg & 4.0 & Yan et al., 2017 & Prp16 / Prp22 \\
\hline & & 5mps, 5mq0 & 3.9 & Fica et al., 2017 & Prp22 \\
\hline & H. sapiens & $5 \mathrm{mqf}$ & 5.9 & Bertram et al., 2017a & Prp22, eIF4A-III, Aquarius \\
\hline & & $5 x j c$ & 3.6 & Zhang et al., 2017 & $\begin{array}{l}\text { Brr2, Prp22, eIF4A-III, } \\
\text { Aquarius }\end{array}$ \\
\hline \multirow[t]{5}{*}{$\mathrm{P}$} & S. cerevisiae & $5 y l z$ & 3.6 & Bai et al., 2017 & Prp22 \\
\hline & & 6bk8 & 3.3 & Liu et al., 2017 & Prp22 \\
\hline & & $6 e x n$ & 3.7 & Wilkinson et al., 2017 & Prp22 \\
\hline & H. sapiens & 6idz & 3.0 & Zhang et al., 2019 & $\begin{array}{l}\text { Brr2, Prp22, eIF4A-III, } \\
\text { Aquarius, }\end{array}$ \\
\hline & & $6 q d v$ & 3.3 & Fica et al., 2019 & $\begin{array}{c}\text { Brr2, Prp22, eIF4A-III, } \\
\text { Aquarius }\end{array}$ \\
\hline \multirow[t]{3}{*}{ ILS } & S. cerevisiae & $5 y 88$ & 3.5 & Wan et al., 2017 & Prp43 \\
\hline & S. pombe & 3jb9 & 3.6 & $\begin{array}{l}\text { Yan et al., 2015) } \\
\text { Hang et al., } 2015\end{array}$ & - \\
\hline & H. sapiens & 6id0, 6id1 & 2.9 & Zhang et al., 2019 & Prp43, Aquarius \\
\hline
\end{tabular}

\subsection{Key players of the spliceosome}

The spliceosome is a highly dynamic machinery and almost none of its components is present throughout the whole splicing cycle. While the vast majority of its constituents are recruited and leave the complex at different stages, key players of the spliceosome can be divided into two groups: U snRNPs and DExH/D-box ATPases. 


\section{Chapter 1: Introduction}

\subsubsection{U snRNPs}

Five U snRNPs (U1, U2, U4, U5 and U6) build the core of the spliceosome and are recruited to the spliceosome in a sequential manner. They are composed of $U$ snRNAs, Sm (Small nuclear ribonucleoprotein) proteins (B/B' D1, D2, D3, E, F and G) and other proteins specific for each U snRNP (Shaw et al., 2008).

In the course of their maturation cycle U1, U2, U4 and U5 snRNAs are transcribed by the RNA polymerase II and are exported to the cytoplasm where the Sm proteins are assembled in a heteroheptameric ring-like structure around the highly conserved Sm binding motif (Plessel et al., 1997). Subsequently, the $\mathrm{m}^{7} \mathrm{G}$-cap of the $U$ snRNP is hypermethylated to a $m_{3} G$ (2,2,7-trimethyl-guanosine) cap, which serves as the signal for reimport into the nucleus (Mattaj, 1986; Narayanan et al., 2004). In the nucleus additional proteins specific for each $U$ snRNP are bound and in the cajal bodies specific nucleotides of the RNA components are modified by methylation or pseudo-uridylation (Darzacq et al., 2002; Jády et al., 2003).

In contrast to the aforementioned U snRNAs, the U6 snRNA is transcribed by the RNA polymerase III and is not exported out of the nucleus (Paule and White, 2000). Instead of a $\mathrm{m}^{7} \mathrm{G}$ cap, U6 snRNA obtains a $\mathrm{Y}^{-}$ monomethyl phosphate cap after transcription, which targets it to the nucleolus, where it undergoes 2'-Omethylation and pseudo-uridylation (Lange and Gerbi, 2000; Singh and Reddy, 1989). Once it leaves the nucleolus the Sm proteins are assembled and the U6 snRNP is targeted to the cajal bodies. Here the U4/U6.U5 tri-snRNP is assembled, which is then competent for the recruitment to the spliceosome.

Being the core of the spliceosome, the U snRNPs assume important roles like 5'SS and branch-site recognition in order to initiate the assembly, which then in turn serve as a docking platform for the binding of the tri-snRNP. Via countless base-pairing interactions with each other but also with the pre-mRNA, they play a vital role in positioning all RNA components of the spliceosome with the final purpose of ensuring both catalytic steps. Despite their central role, not all of them remain constantly present. The dynamic nature of the spliceosome leads to numerous displacements and recruitments of either $U$ snRNPs or other protein factors and these rearrangements need to be tightly timed to follow the sequential order of a splicing cycle. The right orchestration of these events is mainly given by the action of so-called DExH/D-box helicases.

\subsubsection{Spliceosomal RNA helicases}

All RNA helicases, also called DExH/D-box helicases, involved in the spliceosome belong to the helicase superfamily 2 (SF2), which itself belong to one of the six (SF1-6) RNA- and DNA-dependent superfamilies (Leitão et al., 2015). Generally, SF1 and SF2 members are monomeric acting helicases with two RecA-like domains in close juxtaposition and SF3-SF6 helicases assemble in a hexameric toroidal fashion built of 


\section{Chapter 1: Introduction}

either six RecA-like or $\mathrm{AAA}^{+}$(ATPase associated with diverse cellular activities) domains (Iyer et al., 2004). The SF2 members acting in the splicing cycle belong to the subfamily of the DEAD-box, Ski2-like and DEAH-box helicases.

Although these proteins are termed helicases, which means that they unwind double-stranded nucleic acid strands, not all of them fulfill the requirements to be named as such. These proteins act in virtually all RNP metabolism pathways and assume diverse functions like translocation, RNP remodeling, RNA anchoring, stabilizing folding intermediates and even annealing of nucleic acid strands (Bleichert and Baserga, 2007; Schwer, 2001). They all have in common that they act in the context of nucleic acids often under the consumption of NTP and most of them are part of large macromolecular machines like the spliceosome.

DEAD-box, Ski2-like and DEAH-box helicases share a conserved architecture with two juxtaposed RecAlike domains, also called the helicase core. These RecA-like domains harbor at least eight conserved sequence motifs that are crucial for the function of these proteins (Fig. 3a). Motifs I, II, V and VI are

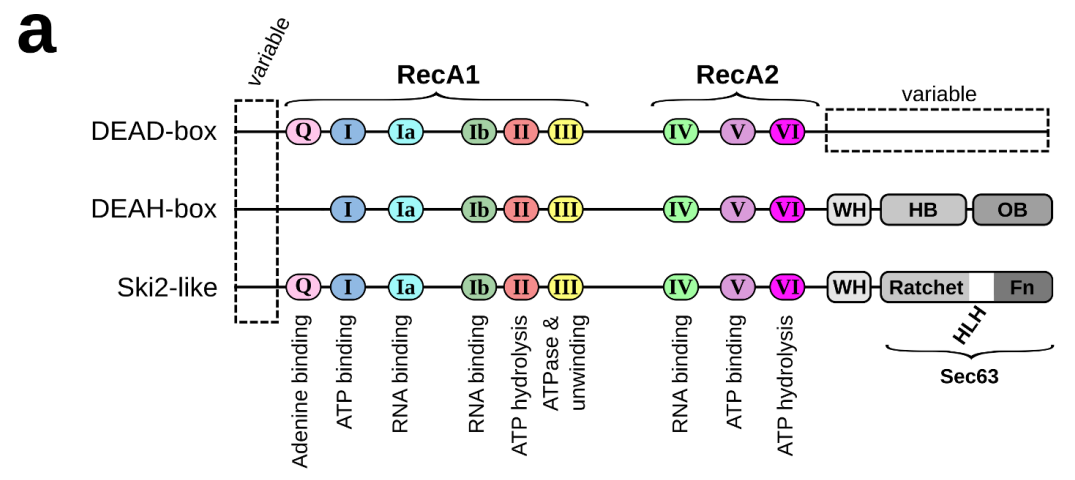

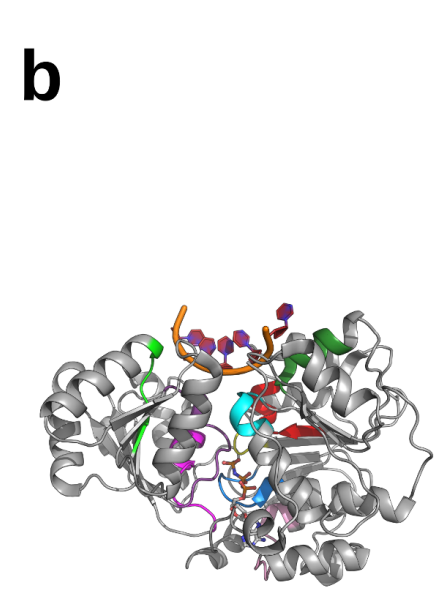

DEAD-box

Vasa - PDBid: $2 \mathrm{db} 3$

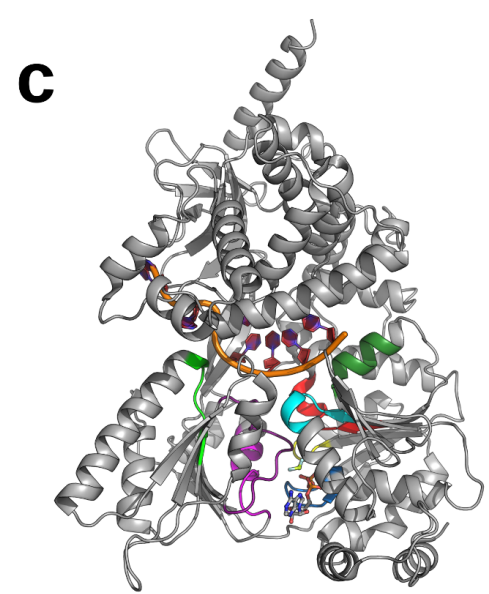

DEAH-box

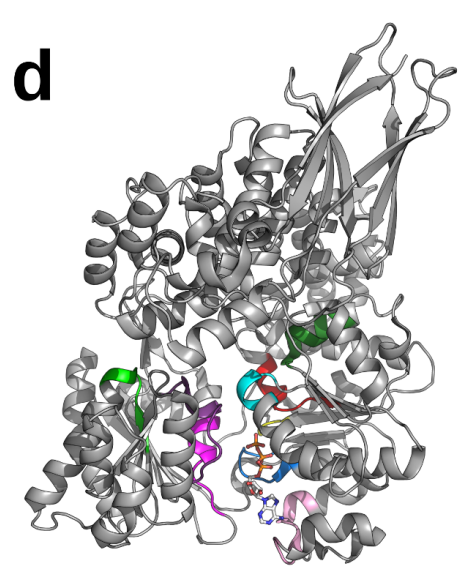

Ski2-like

Figure 3: Conserved sequence motifs of DEAD-box, DEAH-box and Ski2-like helicases. (a) DExH/D-box helicases share at least eight conserved sequence motifs that are allocated on both RecA-like domains. These motifs play a role in ATP binding and hydrolysis, RNA binding as well as in coupling ATP hydrolysis to RNA unwinding. (b-d) When the conserved sequence motifs come into close proximity they form together the nucleotide binding site and the RNA interaction platform. This figure was adopted from Cordin et al. (2012). 


\section{Chapter 1: Introduction}

involved in the binding and hydrolysis of NTP, whereby motifs Ia, Ib and IV are responsible for the interaction with the nucleic acid substrate (Fairman-Williams et al., 2010; Walker et al., 1982). Motif III has been suggested to connect ATP hydrolysis to unwinding (Pause and Sonenberg, 1992; Schwer and Meszaros, 2000). DEAD-box and Ski2-like helicases possess an additional Q-motif, that mediates specificity for ATP (Tanner et al., 2003). These motifs, which are spread over both RecA-like domains, need to come into close proximity in order to form the NTP-binding cleft and the platform for nucleic acid binding (Fig. 3b-d). Additionally, to these sequence motifs, structural features like a $\beta$-hairpin, a hook-loop and a hook-turn have been identified and suggested to play an important role in translocation/unwinding (Prabu et al., 2015; Tauchert et al., 2017; Walbott et al., 2010).

N-terminal flanking regions of these helicases are highly variable in sequence, length and function and assist the helicase in non-catalytic functions like nucleic acid binding or recruitment (Schneider and Schwer, 2001). While the C-terminal domains of DEAD-box helicases are also variable, DEAH-box helicases have a conserved C-terminal architecture composed of a winged-helix (WH), helix-bundle (HB) and oligonucleotide-binding (OB) domain (Fig. 3a). Ski2-like helicases possess as well a C-terminal wingedhelix domain and additionally a Sec63 unit, which is divided into a ratchet, helix-loop-helix (HLH) and fibronection-like (Fn) domain.

While these three SF2 subfamily members that are involved in splicing share a conserved helicase core and interact with nucleic acids in an ATP-dependent manner, they differ strongly in their way of functioning. In the following sections a more detailed focus will be given to these differences and unique features of each subfamily will be highlighted.

\subsubsection{DEAD-box ATPases}

The first steps in splicing are governed by Prp5, Sub2 and Prp28. These proteins belong to the largest subfamily of the SF2 members, the DEAD-box helicases (Fig. 2). Although they share a highly conserved helicase core composed of the RecA-like domains with other members of the SF2 family, they exhibit a high diversity in composition and function of their N- and C-terminal extensions (Fig. 4) (Cordin and Beggs, 2013). Due to the lack of bulky domains flanking the helicase core, DEAD-box helicases are able to adopt a wide range of open conformations with both RecA-like domains distinctly apart from each other (Andersen et al., 2006; Caruthers et al., 2000; Cheng et al., 2005; Zhang et al., 2013). In this conformation the conserved sequence motifs distributed on both RecA-like domains are positioned apart from each other and do not form an active site. In this state the protein is found in a non-productive conformation. In contrast, in the presence of ATP and RNA DEAD-box helicases exhibit a more compact structure with both RecA-like 


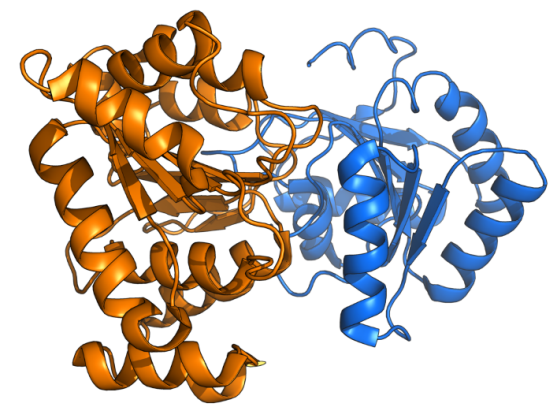

open - non-productive
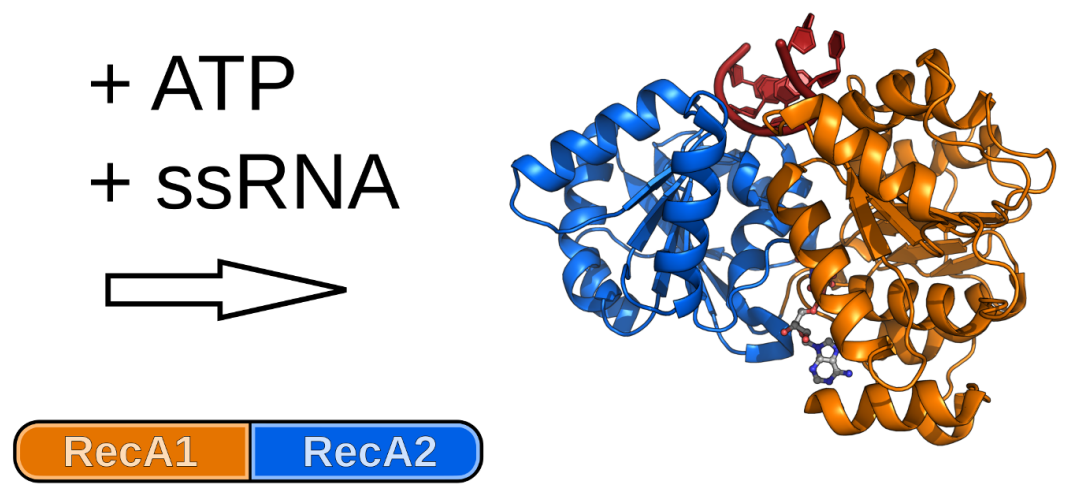

closed - productive

Figure 4: Conformational changes of the DEAD-box helicase eIF4A-III. The DEAD-box helicase eIF4A-III is mainly composed of two RecA-like domains (RecA1: orange; RecA2: blue). In the absence of RNA or nucleotide eIF4A-III adopts a non-productive and open conformation (PDBid: 2hxy). Upon binding of ATP and ssRNA eIF4A-III switches into a closed conformation competent of hydrolyzing ATP and binding RNA (PDBid: 2hyi).

domains in a productive closed conformation (Andersen et al., 2006; Sengoku et al., 2006). Here the conserved sequence motifs are in close proximity and form the active site for nucleotide binding and an interaction stretch for RNA binding (Fig. 3b).

In contrast to most other representatives of the SF2 family, DEAD-box helicases are non-processive duplex unwindases (Pyle, 2008). They disrupt locally short double-stranded regions in a switch-like mechanism by forcing the bound RNA into a pronounced kinked conformation incompatible of forming a duplex (Del Campo and Lambowitz, 2009; Sengoku et al., 2006). Single-stranded regions flanking a duplex facilitate the loading of the helicase onto duplex and in contrast to other SF2 members they do not have any 3' or 5' overhang preference (Bizebard et al., 2004; Rogers et al., 2001; Yang and Jankowsky, 2006). Generally, they bind the backbone of the RNA, making the binding independent of the base sequence and additionally all DEAD-box helicases are able to discriminate between DNA and RNA by directly contacting the 2'OH groups of the ribose moiety (Peck and Herschlag, 1999; Sengoku et al., 2006). Due to the lack of processivity and overhang preference the unwinding mechanism of these helicases differs significantly from the working mechanism of the two remaining SF2 representatives acting in the spliceosome, namely the Ski2-like and DEAH-box ATPases, which will be described in the following sections.

\subsubsection{Ski2-like helicase Brr2}

Brr2 is the only representative of the Ski2-like helicases acting in the spliceosome (Fig. 2). It is loaded onto the U4 snRNA in complex B, where it is responsible for the unwinding of the U4/U6 snRNA duplex (Laggerbauer et al., 1998; Raghunathan and Guthrie, 1998a). As an integral part of the U5 snRNP it is present almost throughout the complete splicing cycle. 
Typical for an SF2 member, Ski2-like helicases possess a conserved helicase core composed of two RecAlike domains, but in contrast to the DEAD-box helicases they are additionally characterized by a conserved C-terminal domain containing a winged-helix (WH), ratchet and helix-loop-helix (HLH) domain (Fig. 3a) (Cordin et al., 2012). Some members like Brr2 exhibit a further C-terminal fibronectin-like (Fn) domain (Fig. 5) (Santos et al., 2012). They unwind their DNA or RNA substrates strictly with a 3'-5' polarity and accomplish the loading of the nucleic acids by binding to a 3' single-stranded overhang (Büttner et al., 2007; Fairman-Williams et al., 2010). A structural study on the Archaeoglobus fulgidus Hel308 suggests that Ski2like helicases unwind nucleic acids duplex by translocating in an ATP-dependent manner on the loaded single strand and thereby disrupting the duplex with the help of a $\beta$-hairpin in the RecA2 domain (Büttner et al., 2007).

Brr2 exhibits a peculiar architecture as it exhibits two helicase cassettes (Santos et al., 2012). Interestingly, only the N-terminal cassette shows ATPase and helicase activity in vitro, whereas the C-terminal cassette lacks such functions. Instead the C-terminal cassette has been proposed to act as a protein-protein interaction unit and to play a role in regulating the activity of the N-terminal domain (van Nues and Beggs, 2001; Santos et al., 2012).

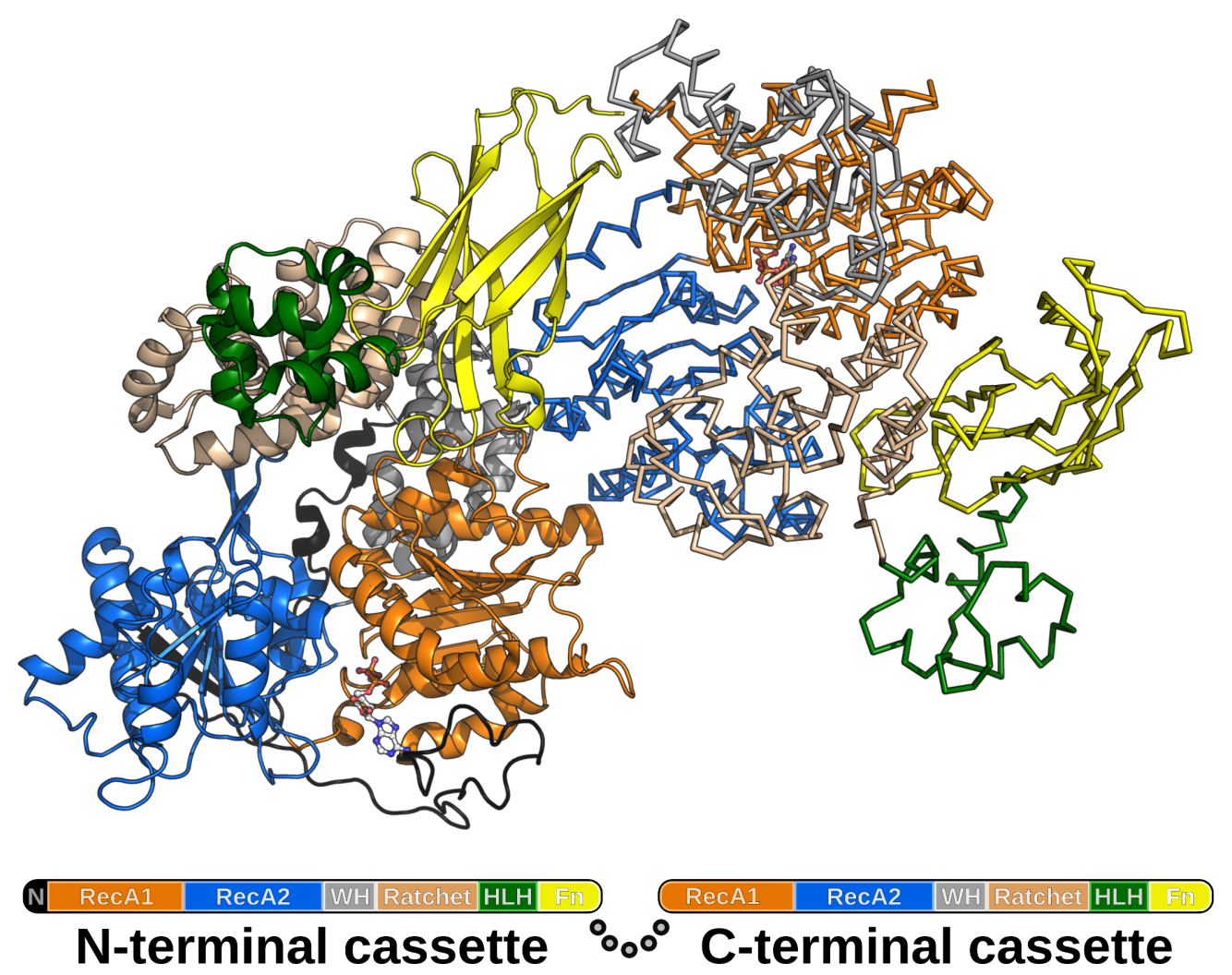

Figure 5: Overall structure of the Ski2-like Brr2. The Ski2-like helicase Brr2 (PDBid: 4f93) is composed of two helicase cassettes. The N-terminal cassette is displayed as a cartoon model, whereas the C-terminal cassette is depicted as a ribbon model. Both cassettes contain a helicase core composed of two RecA-like domains (RecA1: orange; RecA2: blue), a winged-helix (WH: gray), ratchet (wheat), helix-loop-helix (HLH: green) and fibronectin-like (Fn: yellow) domain. The $\mathrm{N}$-terminal extension is shown in black. 


\section{Chapter 1: Introduction}

\subsubsection{DEAH-box ATPases}

DEAH-box ATPases Prp2, Prp16, Prp22 and Prp43 dominate the activation, catalysis and disassembly steps of the splicing cycle. They are composed of a helicase core with two RecA-like domains and a C-terminal domain containing a winged-helix (WH), helix-bundle (HB) and oligonucleotide-binding (OB) domain (Fig. 6) (Tauchert et al., 2017; Walbott et al., 2010). While the C-terminal domains are highly conserved among this subfamily, the N-terminal extensions are very variable and do not only significantly differ in length but also in function (Cordin and Beggs, 2013). The N-terminal domain can serve as an interaction platform for recruitment, RNA binding or for cellular localization (Fouraux et al., 2002; Schneider and Schwer, 2001; Wang and Guthrie, 1998). DEAH-box ATPases lack the Q-motif and therefore are able to hydrolyze any type of NTP (Fig. 3a). While many mostly biochemical studies have verified the importance of the conserved sequence motifs for the function of the spliceosomal DEAH-box ATPases, recent structural investigations have identified additional conserved structural features to play an important role (Campodonico and Schwer, 2002; Schneider et al., 2004; Schwer and Meszaros, 2000; Tanaka and Schwer, 2006). Crystal structures of the DExH-box helicase MLE and Prp43 led to the identification of a hook-loop and a hook-turn, respectively, that were proposed to play a vital role in the processivity of these two proteins

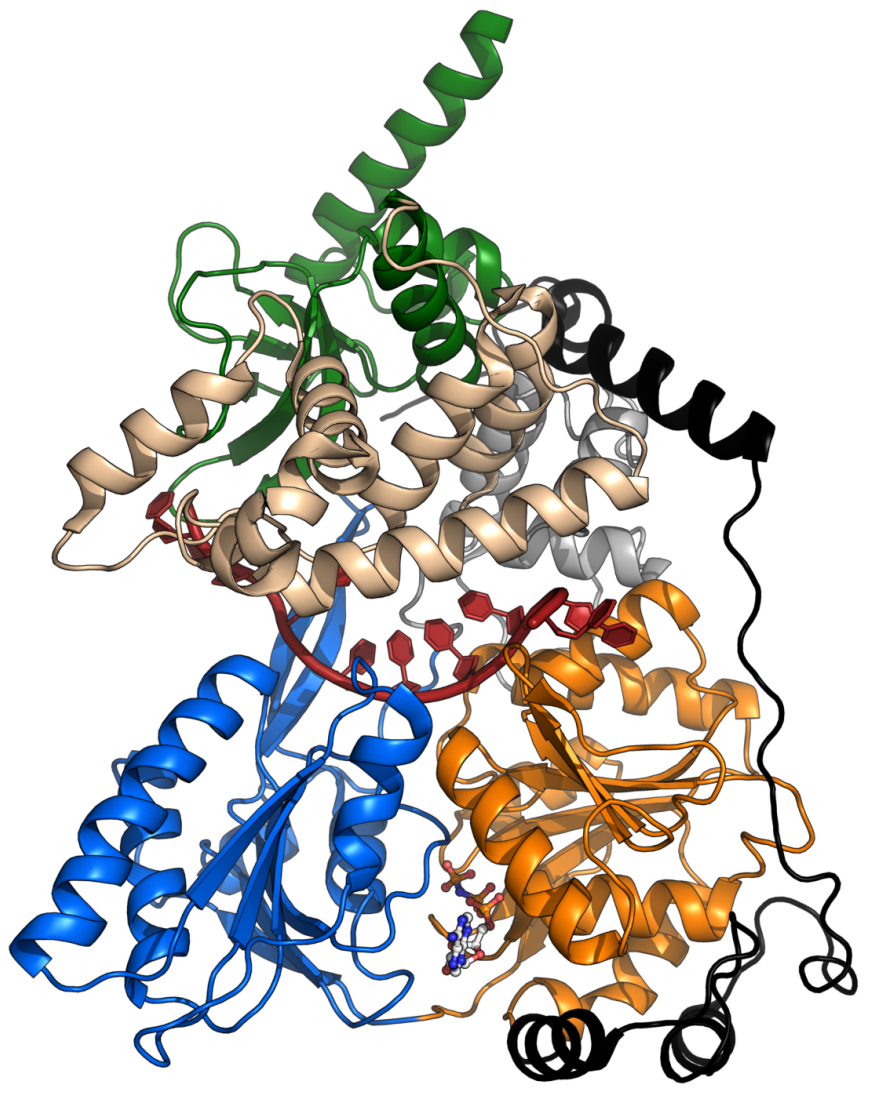

RecA1

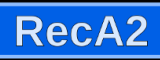

RecA2
Figure 6: Overall structure of the DEAHbox ATPase Prp43. The DEAH-box ATPase Prp43 (PDBid: 5i8q) is composed of a helicase core with two RecA-like domains (RecA1: orange; RecA2 blue) and a Cterminal domain composed of a winged-helix (WH: gray), helix-bundle (HB: wheat) and oligonucleotide-binding (OB: green) domain. An N-terminal extension is depicted in black. Bound nucleotides are sandwiched between the two RecA-like domains. The singlestranded RNA (red) is bound in an RNAbinding tunnel formed between the helicase core and the C-terminal domains. The sugarphosphate backbone of the RNA interacts mainly with the RecA-like domains and the bases face towards the C-terminal domains. 


\section{Chapter 1: Introduction}

(Prabu et al., 2015; Tauchert et al., 2017). DEAH-box ATPases are generally able to interact with DNA as well as with RNA substrates and have the ability to unwind double-stranded nucleic acids with 3'-5' or 5'-3' polarity (Fairman-Williams et al., 2010). It has been shown that single-stranded RNA is bound in a tunnel formed between the helicase core and the C-terminal domains (He et al., 2017; Prabu et al., 2015; Tauchert et al., 2017). The loading of this ssRNA is facilitated by the movement of the C-terminal domains into an open conformation forming a cleft competent for nucleic acid binding, which closes upon RNA binding (Roychowdhury et al., 2019; Tauchert et al., 2017).

It is a longstanding debate how this protein family manages to unwind double-stranded nucleic acids and members of this family have been suggested to work in a processive manner similar to other SF2 members like Ski2-like and NS3 helicases. Processive helicases link translocation along a single-stranded nucleic acid with the disruption of duplexes (Pyle, 2008). However, in order to understand how duplexes are separated it is first required to know how translocation is performed in this subfamily. Additionally, not only recent cryoEM structures but also biophysical studies suggest that especially the spliceosomal DEAH-box ATPase might not require unwindase activity to fulfill their tasks, but instead the mere translocation on a single-stranded RNA might be sufficient (Bao et al., 2017; Fica et al., 2017; Liu et al., 2017; Rauhut et al., 2016; Semlow et al., 2016; Wan et al., 2017; Yan et al., 2016). Due to this reason members of this subfamily have been named DEAH-box ATPases instead of the commonly used term DEAH-box helicases throughout this thesis.

Over the last decade, since the first ADP-bound structures of Prp43 have been published, mainly structural studies on DEAH-box or the closely related DExH-box/RHA ATPases have focused on the elucidation of the translocation mechanism of this family (He et al., 2010, 2017; Prabu et al., 2015; Tauchert et al., 2016, 2017; Walbott et al., 2010). The structural analysis of different nucleotide-bound catalytic states (ADP- and ATPbound) have played a crucial role in the proposition of two models that might describe the translocation mechanism by these proteins: the contraction and the expansion model (He et al., 2017). Based on the contraction model, the DEAH-box ATPases translocate along the single-stranded RNA using small rotational movements of both RecA-like domains in order toggle between states with three and four stacked RNA nucleotides in the binding tunnel. The expansion model however suggests that a so far structurally unknown nucleotide-free state with a much more open helicase core conformation is needed in order to fit a stack of five nucleotides in the RNA binding tunnel and that the nucleotide-dependent repetition of open and closed conformations of the helicase core allows the transition between four and five stacked RNA nucleotides in the binding tunnel. The latter model is inspired by the translocation mechanism of the closely related NS3/NPH-II helicase family (Appleby et al., 2011; Gu and Rice, 2010; Myong et al., 2007).

The main focus of this thesis concentrates on the elucidation of the missing aspects required to fully understand how translocation on a ssRNA in this SF2 subfamily works. In order address this topic the 


\section{Chapter 1: Introduction}

spliceosomal DEAH-box ATPases Prp2 and Prp22 were structurally and biochemically studied during this thesis and will be introduced in the upcoming sections.

\subsection{DEAH-box ATPases examined in this thesis}

\subsubsection{Prp2}

Prp2 plays a critical role during splicing as its recruitment ensures the transition from the activated spliceosome $\left(\mathrm{B}^{\text {act }}\right)$ to the catalytically activated $\left(\mathrm{B}^{*}\right)$ stage of the spliceosome that is then able to perform the first splicing step (Yean and Lin, 1991). Prp2 has been proposed to be recruited to the spliceosome via its Cterminal domains and it is involved in drastic conformational and compositional rearrangements by displacing bound factors and recruiting new ones. Analyses via dcFCCS (dual-color fluorescence crosscorrelation spectroscopy) have provided some insights into the dynamics induced by the recruitment of Prp2 (Ohrt et al., 2012). Using this method it was demonstrated that Cwc24 and Cwc27 dissociate from the complex upon Prp2-mediated catalytic activation. Interestingly, Cwc24 has been shown to be essential for splicing to happen, but its displacement before the first transesterification step indicates that it is not required for the catalysis per se and is regarded just as an assembly factor (Goldfeder and Oliveira, 2008). Other proteins showed not to be completely displaced by the action of Prp2 but their binding affinity was strongly reduced as it is the case for U2-associated SF3a/b proteins Prp11 and Cus1. SF3a/b proteins are bound to pre-mRNA on both sides of the branch site meaning that its destabilization could possibly expose the branch site adenosine and thereby enable its nucleophilic attack at the 5' splice site as also previously suggested in other studies (Gozani et al., 1998; Lardelli et al., 2010). Prp2-mediated rearrangements do not only induce displacements and destabilizations of already bound factors, but its activity during the transition to the
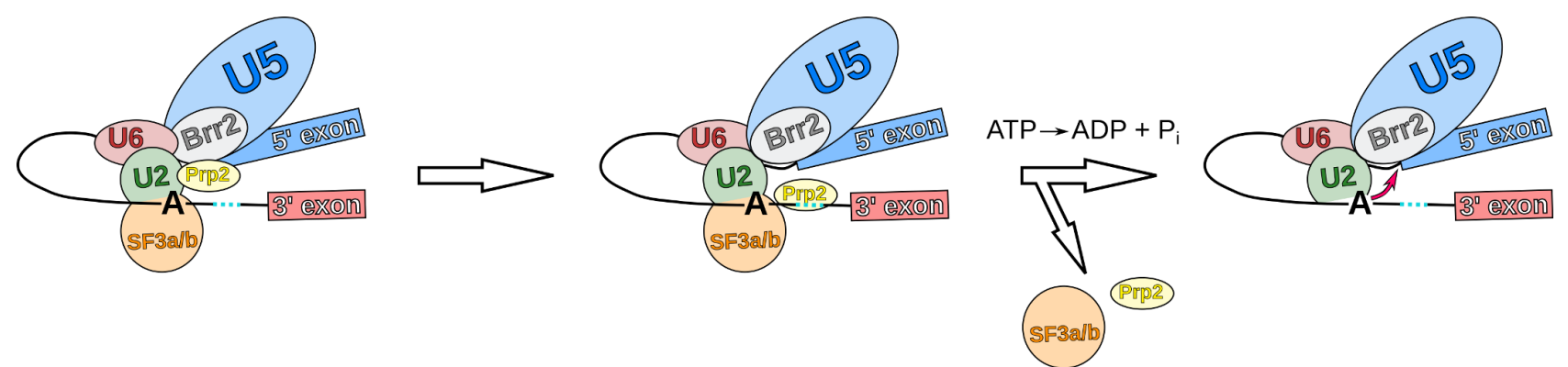

Figure 7: Model of Prp2 action. Prp2 joins the spliceosomal complex at the $\mathrm{B}^{\text {act }}$ complex potentially via initial interactions with the Ski2-like helicase Brr2. Once recruited it binds a segment +23 to +33 downstream of the branchsite in the 3' tail. This region is highlighted as cyan dotted patch on the intron. In the presence of Spp2, the interaction with the RNA stimulates the ATP/translocase activity of Prp2. This leads to major spliceosomal rearrangements, whereby mainly SF3a/b is destabilized, which probably exposes the branch-site adenosine. After its involvement, Prp2 as well leaves the complex. The branch-site adenosine is now available for the first transesterification step. This figure was adopted from Liu et al. (2012). 


\section{Chapter 1: Introduction}

catalytically activated spliceosome also involves stabilizations or/and new recruitments needed for example for the first transesterification step. Yju2 has a very low affinity to spliceosome before Prp2 intervention, but its affinity is strongly enhanced in the catalytically activated complex. Additionally, a strong binding site for the protein Cwc25 is created, recruiting it for the first time to the spliceosome. This results are consistent with previous findings where Cwc25 was shown to be of great importance during the first splicing step after the action of Prp2 as well as the idea that Yju2 might be involved in the recruitment of Cwc25 (Chiu et al., 2009).

In order to fulfill all these rearrangements Prp2 has to interact with the spliceosome and be transiently part of it prior to its remodeling activity. Studies using yeast two-hybrid assays have revealed that Prp2 might interact with Brr2 which was further on verified by pulldown assays (Liu and Cheng, 2012; van Nues and Beggs, 2001). Since Brr2 is an integral part of the spliceosome it has been suggested that Prp2 is recruited to the complex upon binding to Brr2. However, cryo-EM structures of the $\mathrm{B}^{\text {act }}$ complex cannot confirm a direct interaction between Prp2 and Brr2, but only a close spatial proximity (Rauhut et al., 2016; Yan et al., 2016). Additionally, UV cross-linking experiments have proven that Prp2 also interacts with the pre-mRNA downstream of the branch-site (Teigelkamp et al., 1994). The nucleotides interacting with Prp2 have been pinned down to a segment +23 to +33 downstream of branch-site in the 3 ' tail, which is necessary and sufficient to stimulate ATPase activity of Prp2 (Liu and Cheng, 2012). The interaction is position-dependent and seems to have no or low sequence dependency as comparable results were yielded using two different sequences for this region. This is in agreement with the sequence-unspecific binding of ssRNA observed for Prp43, which is likely applicable to the complete subfamily of DEAH-box ATPases (He et al., 2017; Tauchert et al., 2017). The interaction with this region serves as a stimulus for Prp2, inducing its RNAdependent ATPase activity. The destabilization of SF3a/b has been proposed to be due to ATP-driven translocation of Prp2 along the pre-mRNA in 3'-5' direction coming into conflict with SF3a/b's Hsh155, which is associated to the pre-mRNA directly upstream of Prp2's interaction site (Liu and Cheng, 2012; Rauhut et al., 2016; Yan et al., 2016). After destabilization of SF3a/b, the branch-site adenosine is likely exposed and available for the first transesterification step and Prp2 leaves the spliceosomal complex.

\subsubsection{Interplay of Prp2 with its G-patch protein Spp2}

In order to fulfill its function, Prp2 is strictly dependent on the interaction with the G-patch protein Spp2 (Roy et al., 1995; Silverman et al., 2004). G-patch proteins are named after a glycine-rich motif that all members of this protein family harbor, which contains at least six conserved glycine residues (Aravind and Koonin, 1999). While these proteins are absent in bacteria and archaea, they are abundant in eukaryotes and present in some retroviruses, where the G-patch motif is part of RNA- and DNA-binding proteins (Robert- 


\section{Chapter 1: Introduction}

Paganin et al., 2015). Only five G-patch proteins have been identified in yeast and all of them interact with spliceosomal DEAH-box ATPases. Four of them interact with Prp43 (Ntr1, Pfa1, Gno1 and Cmg1) and only one, Spp2, with Prp2 (Guglielmi and Werner, 2002; Heininger et al., 2016; Lebaron et al., 2009; Roy et al., 1995; Tsai et al., 2005).

While the G-patch proteins interacting with Prp43 have been shown to stimulate both, the RNA-independent ATPase as well as the helicase activity of Prp43, Spp2 only increases the ATPase activity of Prp2 when RNA is present (Christian et al., 2014; Tauchert et al., 2017; Warkocki et al., 2015). Up to date, Prp2 is the only spliceosomal DEAH-box helicase not to show any in vitro helicase activity, not even in the presence of its Gpatch protein (Bao et al., 2017; Kim et al., 1992; Warkocki et al., 2015). Such a helicase activity is likely not needed since it targets the single-stranded pre-mRNA at the spliceosomal periphery and Prp2 has been suggested to accomplish its function via translocation rather than unwinding (Liu and Cheng, 2012; Rauhut et al., 2016; Yan et al., 2016). Here, Spp2 plays a critical role in strongly stimulating the ATPase activity of Prp2 once it interacts with the pre-mRNA, since in absence of the G-patch protein Prp2 only exhibits low levels of activity (Warkocki et al., 2015).

It has been shown that Prp2 is recruited to the spliceosome in complexes lacking Spp2, but only upon addition of Spp2 catalytic activation can happen (Warkocki et al., 2015). One the one hand, the G-patch protein induced stimulation could play an important role, but on the other hand, Spp2 could also play a role in proper positioning of Prp2 once recruited. Although cryo-EM structures of the $\mathrm{B}^{\text {act }}$ spliceosome seem to contradict that Prp2 is recruited via Brr2 due to lack of direct contacts in these complexes, it could be feasible that Prp2 initially joins the spliceosome by interacting with Brr2 and is subsequently properly positioned by Spp2 to interact with the pre-mRNA (Liu and Cheng, 2012; van Nues and Beggs, 2001; Rauhut et al., 2016; Yan et al., 2016). Due to the high flexibility of Spp2, the cryo-EM structures, unfortunately, do not provide any structural information about the interaction of Spp2 with either the spliceosome or Prp2. Here, crystal structures of a Prp2-Spp2 complex as well as deeper insights into the biophysical properties of the G-patch in solution could complement the available data and provide a more profound understanding about the interplay between Prp2 and Spp2.

\subsubsection{Prp22}

Prp22 is a DEAH-box ATPase that is responsible for the transition from the complex P to the ILS complex and ensures the release of the mature mRNA out of the spliceosomal complex after intron cleavage (Company et al., 1991; Schwer, 2008). As common for spliceosomal DEAH-box ATPases, Prp22 binds at the periphery of the spliceosome, specifically to the 3' exon and it has been shown that it requires at least 13 


\section{Chapter 1: Introduction}

ribonucleotides downstream from the 3'SS (Liu et al., 2017; Schwer, 2008). Structural and biochemical data are consistent with a model where Prp22 uses translocation on the pre-mRNA to pull on it and thereby promote remodeling of the spliceosome from a distance (Semlow et al., 2016). Although Prp22 is additionally bound to the spliceosome via interactions with Prp8 and Yju2, the interaction with the RNA might play the major role for the attachment since once the pre-mRNA is pulled out/released, Prp22 as well dissociates from the complex (Liu et al., 2017; Semlow et al., 2016; Wilkinson et al., 2017).

Prp22 has also been attributed the function of being able to perform proofreading in order to inhibit splicing of suboptimal 3'SS or to select for alternative splice sites (Mayas et al., 2006; Semlow et al., 2016). The DEAH-box ATPase is already part of the spliceosome in complex $C^{*}$ and the cryo-EM structure of this state exhibits density for 3 nucleotides in the RNA-binding tunnel, which likely correspond to the 3' exon (Fica et al., 2017). At this stage the 3'exon-intron is already docked close to the end of the 5' exon, but the exons are not yet ligated. By pulling on the 3'exon-intron at this stage, Prp22 could compete with the exon ligation event by destabilizing 3'SS and spliceosome interactions that eventually might discard suboptimal 3'SS or ensure the selection of alternative 3’SS.

Similar to all other spliceosomal DEAH-box ATPases, Prp22 is as well stimulated by the presence of RNA, is able to unwind duplexes with a 3'-5' polarity, is not sequence-specific and needs a 3' overhang, but in contrast to Prp43 and Prp2 it has not been shown to require a G-patch-like interaction partner to fulfill its function (Tanaka and Schwer, 2005; Wagner et al., 1998). Prp22 exhibits the longest N-terminal extension of the spliceosomal DEAH-box ATPases, which contains a S1 domain important for spliceosomal function (Schneider and Schwer, 2001). On the one hand, the lack of the need of an additional protein factor to stimulate ATPase/translocase/helicase activity makes Prp22 an interesting target for comparisons with DEAH-box ATPases requiring G-patch proteins for their function, like Prp2 and the most intensively structurally studied spliceosomal DEAH-box ATPase Prp43 (He et al., 2010, 2017; Tauchert et al., 2016, 2017; Walbott et al., 2010). On the other hand, due to the independence of external modulating factors, Prp22 is a more suitable candidate than Prp43 to study mechanistic details that might help to fully understand processivity in this SF2 subfamily. Here, the characterization of structurally undetermined catalytic states (for example states in absence of an adenosine nucleotide) could provide the decisive insights to unravel the translocation mechanism of spliceosomal DEAH-box ATPases. 


\section{Chapter 1: Introduction}

\subsection{Scope - Insights into the translocation mechanism of DEAH-box ATPases}

This thesis mainly concentrates on the structural and biochemical characterization of the two spliceosomal DEAH-box ATPases Prp2 and Prp22. The work on these two proteins lead to two peer-reviewed publications (chapters 2 and 5), one submitted manuscript to a peer-reviewed journal (chapter 3) and a manuscript in preparation (chapter 4).

At the beginning of this thesis no structural data of either Prp2 nor Prp22 was publicly available. Prp43 was the only structurally characterized and by far the most biochemically studied spliceosomal DEAH-box ATPase. However, the exact mechanism of unwinding/translocation of this SF2 subfamily has long remained elusive and mechanistic propositions were primarily driven by comparisons with other SF2 members of the closely related Ski2-like and NS3/NPH-II subfamilies. Recent structural work on Prp43 and the DExH-box ATPase MLE have contributed to a better understanding of how these proteins load and bind ssRNA and have identified new structural features important for processivity, like the hook-loop and hook-turn. While these structures started to unravel structural dynamics needed for a processive function by comparing ATPand ADP-bound states, structural snapshots of spliceosomal DEAH-box ATPases in the absence of any adenosine nucleotide were still missing. In order to complete the structural repertory of catalytic states, Prp22 from Chaetomium thermophilum was crystallized in two different adenosine nucleotide-free states. Using the information of these structures compared with already available structures of Prp43 a more complete model for single-stranded RNA translocation could be postulated.

A second project focused on the elucidation of the functional differences of Prp2 compared with other spliceosomal DEAH-box ATPases and the structural investigation of the Prp2-Spp2 G-patch interaction. Although there were no structural information available of Prp2 at the start of the thesis, sequence alignments already suggested a highly similar architecture of the catalytic unit compared to Prp43. But in contrast to Prp43, Prp2 is unable to unwind duplex RNAs. Despite the high similarity, minor differences not obvious at a sequence level, seem to differently regulate the function of these DEAH-box ATPases. The investigation of such differences might additionally provide insights into more fundamental aspects of the processive function of this protein family. In order to identify these subtle differences, complexes already characterized for Prp43 (ADP-bound as well as ATP- and RNA-bound complexes) were crystallized for Prp2 from Chaetomium thermophilum. Additionally, the interaction between Prp2 and the G-patch motif of Spp2 was investigated on a structural manner in order to unveil how this interaction influences Prp2 activity and how the bound G-patch is able to adapt to the conformational dynamics of the ATPase during translocation. 


\title{
Chapter 2: Crystal structure of the spliceosomal DEAH- box ATPase Prp2
}

\section{Crystal structure of the spliceosomal DEAH-box ATPase Prp2}

\author{
Andreas Schmitt ${ }^{\S 1}$, Florian Hamann ${ }^{\S 1}$, Piotr Neumann ${ }^{1}$, Ralf Ficner ${ }^{1 *}$ \\ ${ }^{1}$ Department of Molecular Structural Biology, Institute for Microbiology and Genetics, GZMB, Georg- \\ August-University Göttingen, Justus-von-Liebig-Weg 11, 37077 Göttingen, Germany \\ ${ }^{\S}$ These authors contributed equally. \\ *To whom correspondence should be addressed: rficner@gwdg.de
}

\section{Author contributions:}

A.S.: Experimental design, molecular cloning, protein expression and purification, crystallization, data collection and processing, structure determination, structure refinement

F.H.: Experimental design, molecular cloning, protein expression and purification, crystallization, data collection and processing, structure determination, structure refinement and analysis, Isothermal titration calorimetry (ITC) measurements, data analysis, preparation of figures and tables, writing - original draft, writing - review and editing

P.N.: Data collection and processing, structure determination, structure refinement, writing - review and editing

R.F.: Experimental design, crystal structure analysis, data analysis, writing - review and editing, supervision, project leader 
Chapter 2: Crystal structure of the spliceosomal DEAH-box ATPase Prp2

\section{electronic reprint}

STRUCTURAL

BIOLOGY

ISSN: 2059-7983

journals.iucr.org/d

Crystal structure of the spliceosomal DEAH-box ATPase Prp2

Andreas Schmitt, Florian Hamann, Piotr Neumann and Ralf Ficner

Acta Cryst. (2018). D74, 643-654

\section{- IUCr Journals}

CRYSTALLOGRAPHY JOURNALS ONLINE

This open-access article is distributed under the terms of the Creative Commons Attribution Licence

http://creativecommons.org/licenses/by/2.0/uk/legalcode, which permits unrestricted use, distribution, and reproduction in any medium, provided the original authors and source are cited. 


\section{Chapter 2: Crystal structure of the spliceosomal DEAH-box ATPase Prp2}

research papers

STRUCTURAL BIOLOGY

ISSN 2059-7983

Received 5 January 2018 Accepted 25 April 2018

Edited by M. Rudolph, F. Hoffmann-La Roche Ltd, Switzerland

₹ These authors contributed equally.

Keywords: spliceosome; RNA helicase; DEAH-box; Prp2.

PDB references: DEAH-box helicase Prp2, 6fa9; complex with ADP, crystal form 1, 6fac; crystal form 2, 6faa; crystal form 3, 6fa5

Supporting information: this article has supporting information at journals.iucr.org/d

\section{Crystal structure of the spliceosomal DEAH-box ATPase Prp2}

\author{
Andreas Schmitt,‡ Florian Hamann,‡ Piotr Neumann and Ralf Ficner* \\ Department of Molecular Structural Biology, Institute of Microbiology and Genetics, GZMB, Georg-August-University \\ Göttingen, Justus-von-Liebig-Weg 11, 37077 Göttingen, Germany. *Correspondence e-mail: rficner@uni-goettingen.de
}

The DEAH-box ATPase Prp2 plays a key role in the activation of the spliceosome as it promotes the transition from the $\mathrm{B}^{\text {act }}$ to the catalytically active $\mathrm{B}^{*}$ spliceosome. Here, four crystal structures of $\operatorname{Prp} 2$ are reported: one of the nucleotide-free state and three different structures of the ADP-bound state. The overall conformation of the helicase core, formed by two RecA-like domains, does not differ significantly between the ADP-bound and the nucleotide-free states. However, intrinsic flexibility of Prp2 is observed, varying the position of the C-terminal domains with respect to the RecA domains. Additionally, in one of the structures a unique ADP conformation is found which has not been observed in any other DEAH-box, DEAD-box or NS3/NPH-II helicase.

\section{Introduction}

In eukaryotic cells, precursor messenger RNAs (pre-mRNAs) may contain noncoding intervening sequences (introns) that have to be removed by splicing prior to nuclear export and translation of the mRNAs. The process of pre-mRNA splicing is carried out by a highly dynamic multi-megadalton ribonucleoprotein (RNP) complex, the spliceosome, which catalyzes two sequential transesterification reactions (Will \& Lührmann, 2011; Hoskins \& Moore, 2012; Matera \& Wang, 2014). For each intron to be excised, the spliceosome assembles stepwise onto the pre-mRNA from five small nuclear ribonucleoprotein particles (snRNPs), named U1, U2, U4/U6 and U5, and a large number of non-snRNP proteins (Wahl et al., 2009). Briefly, the assembly starts with the U1 snRNP binding to the $5^{\prime}$ splice site of the pre-mRNA and the U2 snRNP interacting with the branch-point sequence (BPS), resulting in the formation of the spliceosomal A complex. Subsequently, the pre-assembled tri-snRNP consisting of the U4/U6 and U5 snRNPs is recruited to the spliceosome, leading to the formation of the catalytically inactive $\mathrm{B}^{\text {act }}$ complex. In the subsequent step of the splicing cycle, the catalytically active $\mathrm{B}^{*}$ complex is formed which facilitates the first transesterification reaction. After this, complex $\mathrm{C}$ is made by further remodelling and the second transesterification reaction takes place. Finally, the spliceosome is disassembled, leading to release of the spliced mRNA and the intron lariat.

During assembly, the splicing reaction and disassembly, the spliceosome undergoes large compositional and conformational changes, including the remodelling of RNA-RNA, RNA-protein and protein-protein interactions (Wahl et al., 2009). These rearrangements are mainly driven by eight $\mathrm{DE} x \mathrm{D} / \mathrm{H}$-box ATPases which use the energy from ATP hydrolysis to unwind dsRNA and/or remodel RNA-protein interactions (Cordin et al., 2012; Ding \& Pyle, 2012; Ozgur et al., 2015). These helicases belong to helicase superfamily 2 


\section{Chapter 2: Crystal structure of the spliceosomal DEAH-box ATPase Prp2}

\section{research papers}

Table 1

Data-collection and refinement statistics

\begin{tabular}{|c|c|c|c|c|}
\hline & NT-free & CF1 & $\mathrm{CF} 2$ & CF3 \\
\hline \multicolumn{5}{|l|}{ Data collection } \\
\hline Space group & $P 2_{1} 2_{1} 2_{1}$ & $C 2$ & $P 2_{1} 2_{1} 2_{1}$ & $P 2_{1} 2_{1} 2_{1}$ \\
\hline \multicolumn{5}{|l|}{ Unit-cell parameters } \\
\hline$a(\AA)$ & 50.6 & 122.7 & 50.3 & 48.2 \\
\hline$b(\AA)$ & 114.1 & 67.3 & 114.2 & 116.4 \\
\hline$c(\AA)$ & 120.7 & 95.6 & 117.8 & 118.2 \\
\hline$\beta\left({ }^{\circ}\right)$ & 90 & 106.5 & 90 & 90 \\
\hline $\mathrm{X}$-ray source & BL14.1, BESSY & $\begin{array}{l}\text { ID23-2, ESRF } \\
\text { Grenoble }\end{array}$ & BL14.1, BESSY & $\begin{array}{l}\text { ID23-2, ESRF } \\
\text { Grenoble }\end{array}$ \\
\hline Resolution range ( $\left({ }^{\prime}\right)$ & $\begin{array}{l}41.46-2.60 \\
\quad(2.70-2.60)\end{array}$ & $\begin{array}{l}46.41-2.05 \\
\quad(2.15-2.05)\end{array}$ & $\begin{array}{l}46.00-1.97 \\
\quad(2.07-1.97)\end{array}$ & $\begin{array}{l}44.64-2.30 \\
\quad(2.44-2.30)\end{array}$ \\
\hline No. of unique reflections & 22177 & 46873 & 48358 & 30007 \\
\hline Completeness (\%) & $99.8(99.9)$ & 99.5 (99.6) & $99.0(99.3)$ & $99.1(97.9)$ \\
\hline$R_{\text {merge }}(\%)$ & $7.0(84.0)$ & $3.9(79.4)$ & $9.2(84.6)$ & $7.9(85.3)$ \\
\hline Average $I / \sigma(I)$ & $20.43(2.34)$ & $20.31(2.19)$ & $13.43(1.77)$ & $13.78(1.91)$ \\
\hline Multiplicity & $4.86(4.98)$ & $4.08(4.2)$ & $3.74(3.6)$ & $4.28(4.35)$ \\
\hline $\mathrm{CC}_{1 / 2}(\%)$ & $99.9(82.5)$ & $99.9(83.4)$ & $99.7(62.7)$ & $99.8(65.3)$ \\
\hline Wilson $B\left(\AA^{2}\right)$ & 50.97 & 44.79 & 24.74 & 49.4 \\
\hline \multicolumn{5}{|l|}{ Refinement } \\
\hline Resolution (Å) & $\begin{array}{l}41.46-2.70 \\
\quad(2.85-2.70)\end{array}$ & $\begin{array}{l}46.41-2.05 \\
\quad(2.09-2.05)\end{array}$ & $\begin{array}{l}46.00-1.97 \\
\quad(2.01-1.97)\end{array}$ & $\begin{array}{l}\text { 44.64-2.30 } \\
\quad(2.38-2.30)\end{array}$ \\
\hline No. of reflections & 22168 & 46832 & 48349 & 29999 \\
\hline$R_{\text {work }}(\%)$ & $23.00(32.89)$ & $21.60(35.53)$ & $18.75(29.83)$ & $19.79(31.56)$ \\
\hline$R_{\text {free }}(\%)$ & $27.59(33.99)$ & $24.41(37.63)$ & $22.42(32.82)$ & $24.54(35.45)$ \\
\hline Total No. of atoms & 5020 & 5384 & 5553 & 5047 \\
\hline Protein residues & 636 & 653 & 637 & 635 \\
\hline Water molecules & 42 & 222 & 445 & 59 \\
\hline Ligand molecules & 2 & 6 & 7 & 3 \\
\hline \multicolumn{5}{|l|}{ R.m.s. deviations } \\
\hline Bond lengths $(\AA)$ & 0.004 & 0.010 & 0.005 & 0.006 \\
\hline Bond angles $\left({ }^{\circ}\right)$ & 0.852 & 1.476 & 1.051 & 0.792 \\
\hline \multicolumn{5}{|l|}{ Mean $B$ factors $\left(\AA^{2}\right)$} \\
\hline Protein & 70.2 & 62.7 & 29.3 & 58.2 \\
\hline ADP & - & 58.6 & 25.7 & 57.4 \\
\hline \multicolumn{5}{|l|}{ Ramachandran statistics } \\
\hline Favoured (\%) & 97.15 & 97.55 & 98.13 & 97.00 \\
\hline Allowed (\%) & 2.69 & 2.45 & 1.72 & 3.00 \\
\hline Outliers (\%) & 0.16 & 0.0 & 0.16 & 0.00 \\
\hline PDB code & $6 \mathrm{fa} 9$ & 6fac & 6faa & $6 \mathrm{fa} 5$ \\
\hline
\end{tabular}

remodelling of the spliceosome by Prp2 creates a high-affinity binding site for the NineTeen Complex (NTC) proteins Yju2 and Cwc25, both of which seem to play an important role in stabilizing the pre-mRNA in a suitable conformation for the first transesterification step (Chiu et al., 2009; Ohrt et al., 2012; Krishnan et al., 2013). More recent data also suggest that Prp2 might be directly or indirectly involved in destabilization of the U2/U6 helix Ia and of putative base triples (Wlodaver \& Staley, 2014). The activated spliceosome subsequently undergoes the first catalytic step, generating the $\mathrm{C}$ complex.

Recent cryo-EM structures of the $\mathrm{B}^{\text {act }}$ complex contain Prp2; a homology model derived from the Prp43 crystal structure was used to interpret the cryoEM map for Prp2 (Rauhut et al., 2016; Yan et al., 2016). However, the local resolution of these cryo-EM maps for Prp2 is 7.5 or $9.9 \AA$; hence, they provide only limited information regarding the Prp2 structure (Ficner et al., 2017).

Here, we report four crystal structures of Prp2 from Chaetomium thermophilum at resolutions of 1.97 , 2.05, 2.3 and $2.7 \AA$, representing the first high-resolution atomic models of Prp2. One of the crystal structures corresponds to the nucleotide-free state, but it contains a sulfate ion bound in the catalytic centre that probably mimics

(SF2) and share a common fold consisting of two RecA-like motifs which together form the helicase core (FairmanWilliams et al., 2010). In DE $x \mathrm{D} / \mathrm{H}-$ box helicases, the RecA domains harbour eight conserved sequence motifs named I, Ia, $\mathrm{Ib}$, II, III, IV, V and VI, which are involved in RNA and/or nucleotide binding, with motif II carrying the name-giving consensus sequence $\mathrm{DE} x \mathrm{D} / \mathrm{H}$ (Cordin et al., 2006). Additional domains located $\mathrm{C}$-terminal to the helicase core have been shown to act as a binding platform for interaction partners or have a regulatory effect on the ATPase activity of the helicase (Cordin \& Beggs, 2013; Kudlinzki et al., 2012).

The transition to the catalytically active $\mathrm{B}^{*}$ complex is promoted by the DEAH-box ATPase Prp2 together with its cofactor Spp2 (King \& Beggs, 1990; Roy et al., 1995; Kim \& Lin, 1996; Silverman et al., 2004). Prp2 facilitates the liberation of the $5^{\prime}$ splice site and the branch-site adenosine by destabilization of the SF3a and SF3b protein complexes, which are bound to the intron near the branch site. As a consequence, the branch-site adenosine becomes available for subsequent nucleophilic attack of the $5^{\prime}$ splice site (Warkocki et al., 2009; Lardelli et al., 2010; Ohrt et al., 2012; Bao et al., 2017). Moreover, it has been demonstrated that the the remaining phosphate ion after the hydrolysis of ATP and the release of ADP. The other three structures represent Prp2 with a bound ADP molecule, one of which reveals a significantly different ADP conformation.

\section{Materials and methods \\ 2.1. Gene expression and protein production}

Prp2 from C. thermophilum (ctPrp2) was identified by the NCBI BLAST search tool (GenBank EGS18341.1) and the gene encoding a truncated version of the protein comprising residues 270-921 was amplified by PCR from a total DNA preparation. The PCR product was cloned into the IBA Stargate pASG-IBA-25 vector according to the manufacturer's protocol. The GST-fused recombinant protein was expressed in Escherichia coli Rosetta 2 (DE3) cells at $16^{\circ} \mathrm{C}$. The cells were disrupted using a fluidizer (Microfluidics) in $50 \mathrm{~m} M$ HEPES-NaOH pH 7.5, $500 \mathrm{~m} M \mathrm{NaCl}, 5 \%(v / v)$ glycerol, $10 \mathrm{~m} M$ EDTA, $1 \mathrm{~m} M$ DTT and the lysate was clarified by centrifugation for $30 \mathrm{~min}$ at $30000 \mathrm{~g}$. The protein was purified at $20^{\circ} \mathrm{C}$ on a Glutathione Sepharose column (GE 


\section{Chapter 2: Crystal structure of the spliceosomal DEAH-box ATPase Prp2}

\section{research papers}

Healthcare) in $50 \mathrm{~m} M$ HEPES- $\mathrm{NaOH}$ pH 7.5, $500 \mathrm{~m} M \mathrm{NaCl}$, $5 \%(v / v)$ glycerol, $10 \mathrm{~m} M$ EDTA, $1 \mathrm{~m} M$ DTT. A wash step with binding buffer supplemented with $2 M \mathrm{LiCl}$ was included to remove bound nucleic acids, followed by elution of the bound fusion protein with $30 \mathrm{~m} M$ reduced glutathione and cleavage of the GST tag with PreScission protease. Further purification was achieved using a Superdex 200 gel-filtration column, and residual GST was subsequently removed using a Glutathione Sepharose column. A check for contamination by nucleic acids was performed by measuring the absorbance of the protein solution at 260 and $280 \mathrm{~nm}$. The protein was concentrated to $15 \mathrm{mg} \mathrm{ml}^{-1}$ using an Amicon Ultra centrifugal concentrator (Merck) in $10 \mathrm{~m} M$ HEPES-NaOH pH 7.5, $200 \mathrm{~m} M \mathrm{NaCl}$, $2 \mathrm{~m} M \mathrm{MgCl}_{2}, 1 \mathrm{~m} M$ DTT. A shortened construct comprising residues 286-921 was used for the crystallization of crystal form 3 (CF3), but all purification steps were performed as described above.

\subsection{Crystallization}

Crystallization trials were performed with $\operatorname{Prp} 2$ at a concentration of $2 \mathrm{mg} \mathrm{ml}^{-1}(27.4 \mu M)$ and incubated with a tenfold molar excess of ADP or AMPPCP $(274 \mu M)$. Crystals were grown at $293 \mathrm{~K}$ by sitting-drop vapour diffusion using drops consisting of $1 \mu 1$ protein and $1 \mu 1$ reservoir solution. Nucleotide-free crystals (NT-free) were obtained in $100 \mathrm{mM}$ Tris- $\mathrm{HCl} \mathrm{pH} \mathrm{7.5,} 200 \mathrm{mM} \mathrm{Li} \mathrm{SO}_{4}, 15 \%(w / v)$ PEG 8000; crystal form 1 (CF1) in $100 \mathrm{~m} M$ HEPES-MOPS- $\mathrm{NaOH}$ pH 7.5, $60 \mathrm{mM} \mathrm{MgCl} / \mathrm{CaCl}_{2}, 20 \%(w / v)$ ethylene glycol, $10 \%(w / v)$ PEG 8000; CF2 in $100 \mathrm{~m} M$ Tris- $\mathrm{HCl}$ pH 7.5, $200 \mathrm{~m} M \mathrm{NaCl}$, $15 \%(w / v)$ PEG 8000 and $\mathrm{CF} 3$ in $100 \mathrm{~m} M$ HEPES-NaOH $\mathrm{pH}$ 7, $250 \mathrm{mM} \mathrm{NaCl}, 25 \%(w / v)$ PEG 6000. Crystals were obtained after $2-5 \mathrm{~d}$.

\subsection{Data collection and processing}

The crystals were transferred into mother liquor containing $5 \%(v / v)$ glycerol as well as $10 \%(v / v)$ PEG 400 for CF1 and $\mathrm{CF} 3$, and $10 \%(v / v)$ glycerol and $20 \%(v / v)$ PEG 400 for NTfree and CF2 prior to flash-cooling in liquid nitrogen. Oscillation images for NT-free and CF2 were collected on BL14.1 operated by the Helmholtz-Zentrum Berlin (HZB) at the BESSY II electron-storage ring, Berlin-Adlershof, Germany (Mueller et al., 2012), and the CF1 and CF3 data sets were collected on ID23-2 operated by the European Synchrotron Radiation Facility (ESRF). Data were processed with the XDS package using a minimum $I / \sigma(I)$ of 1.7 and a minimum $\mathrm{CC}_{1 / 2}$ of $60 \%$ for the highest resolution shell as cutting criteria (Kabsch, 2010).

\subsection{Structure solution, refinement and analysis}

The crystallographic phase problem was solved by molecular replacement, which was first performed for CF2 with Phaser (McCoy et al., 2007) using chain $A$ of scPrp43 (PDB entry 2xau; Walbott et al., 2010) as a search model. The NTfree, CF1 and CF3 structures were solved using the CF2 structure as a search model for molecular replacement. Manual model building was conducted with Coot employing both difference electron-density maps $\left(2 m F_{\mathrm{o}}-D F_{\mathrm{c}}, m F_{\mathrm{o}}-D F_{\mathrm{c}}\right)$ and simulated-annealing composite OMIT maps (Emsley et al., 2010). Refinements were performed with PHENIX, including TLS, weight optimization and bulk-solvent optimization (Adams et al., 2010). The quality of the final models was assessed using the validation tools in PHENIX and MolProbity (Chen et al., 2010). Structure superposition was performed with LSQMAN (Kleywegt, 1996). The figures were prepared with PyMOL (v.1.8; Schrödinger). For complete data-collection and refinement statistics, see Table 1.

\subsection{Isothermal titration calorimetry (ITC)}

ITC experiments for the binding of ADP and AMPPCP to Prp2 were performed on a MicroCal VP-ITC (Malvern) using a Prp2 concentration in the cell of $10 \mu M$ and ADP/AMPPCP concentrations of $100 \mu M$ in the syringe. The reaction buffer consisted of $20 \mathrm{~m} M$ HEPES-NaOH pH 7.5, $200 \mathrm{~m} M \mathrm{NaCl}$, $5 \%$ glycerol, $2 \mathrm{mM} \mathrm{MgCl} 2$. Each measurement consisted of an initial $6 \mu \mathrm{l}$ injection and 19 injections with a volume of $14 \mu \mathrm{l}$ injected at a speed of $1 \mu \mathrm{s}^{-1}$. The binding was monitored at $20^{\circ} \mathrm{C}$ and the interval between each injection was set to $250 \mathrm{~s}$. Data integration was performed with NITPIC, integrated data were processed and analyzed with SEDPHAT, and GUSSI was used for the final representation of the data (Houtman et al., 2007; Keller et al., 2012; Brautigam, 2015).

\section{Results \\ 3.1. Structure of Prp2}

Since all of our attempts to crystallize the spliceosomal DEAH-box ATPase Prp2 from Saccharomyces cerevisiae or Homo sapiens failed, the orthologue of Prp2 from the ascomycete $C$. thermophilum (ctPrp2) was identified and cloned, as proteins from this thermophilic eukaryotic organism may have a higher tendency to crystallize (Amlacher et al.,2011). Recent studies of the closely related DEAH-box helicase Prp43 from C. thermophilum have proven that proteins from this organism are suitable candidates for structural investigations of spliceosomal helicases (Tauchert et al., 2016, 2017). ctPrp2 exhibits amino-acid sequence identities of 44.1 and $49.2 \%$ to yeast Prp2 (scPrp2) and human Prp2 (hsPrp2), respectively. However, full-length ctPrp2 was not soluble after overproduction in $E$. coli; hence, an $\mathrm{N}$-terminally truncated version of ctPrp2 comprising residues 270-921 was cloned, expressed, purified and crystallized. Notably, the missing $269 \mathrm{~N}$-terminal residues were known to not be essential for Prp2 function in yeast (Edwalds-Gilbert et al., 2004). Four different crystal structures of ctPrp2 were obtained: one of the nucleotide-free state and three different crystal forms of the ADP-bound state (Table 1). The nucleotide-free structure was the result of crystallization trials in the presence of the nonhydrolysable ATP analogue AMPPCP and is referred to here as NT-free, whereas the nucleotide-bound structures are denoted crystal forms 1-3 (CF1-CF3).

Full-length Prp2 consists of six domains, five of which are present in the ctPrp2 structure (Fig. 1a) as the 269-residue 
$\mathrm{N}$-terminal domain is absent in the truncated protein used for crystallization. The helicase core comprises two RecA-like domains (referred to as RecA1 and RecA2; Fig. 1a). RecA1 contains the conserved Walker motif A (P-loop) between residues 320 and 327 . RecA 2 contains a prominent antiparallel $\beta$-hairpin that protrudes out of the RecA2 domain. The winged-helix (WH) and helix-bundle (HB) domains are located C-terminally to RecA2; both are homologous to the corresponding domains in Prp43 and the Ski2-like DNA helicase Hel308 (Büttner et al., 2007; Richards et al., 2008). The helix-bundle domain was previously denoted as a ratchet domain and was named according to its previously proposed function of acting as a ratchet in dsDNA or dsRNA unwinding (Walbott et al., 2010; He et al., 2010); this however turned out not to be the case, as shown for other RHA and DEAH-box proteins (Prabu et al., 2015; Tauchert et al., 2017). The C-terminal domain includes a five-stranded $\beta$-barrel exhibiting an oligonucleotide-binding fold (OB-fold; Murzin, 1993). These five domains are common to all spliceosomal DEAHbox helicases.

In comparison to the known structure of the DEAH-box helicase Prp43, the fold of Prp2 is very similar, with the exception of the N-terminal extension (Fig. 2). In scPrp43 the $\mathrm{N}$-terminal extension wraps around the RecA1 domain and the $\mathrm{N}$-terminal residues bind to the $\mathrm{C}$-terminal domains (Walbott et al., 2010; He et al., 2010). In contrast, in Prp2 the 26 residues $\mathrm{N}$-terminal to the RecA1 domain form a long $\alpha$-helix that protrudes from the globular protein molecule. The first three turns of this helix superimpose well with the corresponding helix in Prp43. However, in Prp43 this helix terminates after 13 residues because of a helix-breaking proline at position 81 .

\subsection{Structural flexibility of the C-terminal domains}

Superpositions of the four Prp2 crystal structures presented here reveal distinguishable differences between their conformations, resulting in overall root-mean-square deviations (r.m.s.d.s) calculated between backbone $\mathrm{C}^{\alpha}$ atoms (residues 286-920) that range between 0.81 and $1.70 \AA$ (the average r.m.s.d. based on six superpositions is $1.37 \AA$ ). This is significantly larger than the backbone r.m.s.d.s calculated separately for the RecA2 domain (residues 471-595 and 611-652), the helicase core comprising both RecA-like domains (residues 286-595 and 611-652), and the C-terminal domains (WH, HB and OB; residues $653-920$ ), which range from 0.50 to $0.86 \AA$ (average $0.65 \AA$ ), from 0.66 to $1.24 \AA$ (average $0.97 \AA$ ) and from 0.44 to $1.03 \AA$ (average $0.86 \AA$ ), respectively. Thus, keeping the RecA2 domains of the four compared Prp2 structures superimposed, the observed differences in the positions of their C-terminal domains (CTDs; WH, HB and $\mathrm{OB}$ ) relative to the helicase core become more clear (Fig. 3a).

The observed movement of the C-terminal domains (CTDs) with respect to the helicase core results in a different number of interdomain interactions (Supplementary Table S1). Interestingly, ten of these interactions, involving residues from the winged-helix domain (Supplementary Table S1, Fig. 3b), are conserved among the four analyzed Prp2 structures regardless of the movement of the CTDs. The residues taking part in these conserved interactions are Arg657, Pro653 and Glu654,

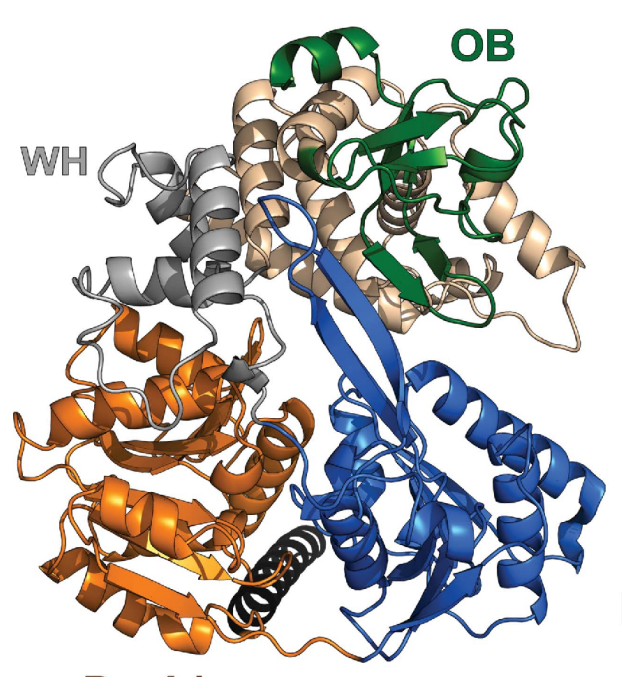

RecA1

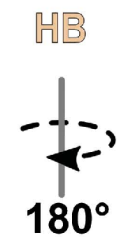

RecA2

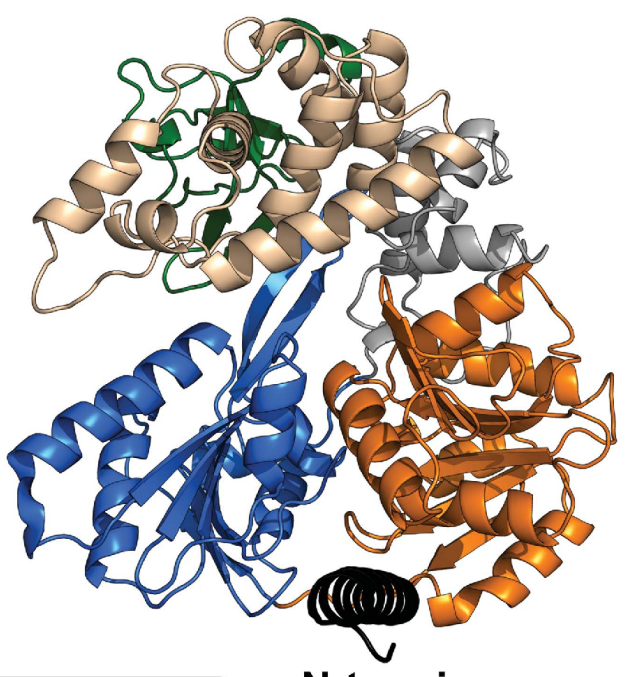

N-terminus

Figure 1

Overall structure of Prp2 from C. thermophilum. The model of ctPrp2 is depicted as a cartoon. The remaining amino acids from the truncated N-terminal extension (270-296) are shown in black, the RecA1 domain (297-475) is in orange, the RecA2 domain (476-652) is in blue, the winged-helix domain (WH; 653-720) is in grey, the helix-bundle domain (HB; 721-852) is in wheat and the oligonucleotide-binding fold (OB; 853-920) is in green. 


\section{Chapter 2: Crystal structure of the spliceosomal DEAH-box ATPase Prp2}

\section{research papers}

which form hydrogen bonds to Asn596 and Thr652 from the RecA2 domain; Pro719, Gly716, Glu654 and Asp679, which form polar contacts with Arg380, Arg401, Arg423 and Tyr461 from the RecA1 domain; and Arg423 and Lys435, which form salt bridges with Asp682 and Asp679 located in the RecA2 domain, respectively. This network of interactions between the winged-helix (WH) domain and the helicase core anchors the CTDs to the adjacent surface of the RecA1 domain. As a consequence, any movement of the RecA1 domain relative to the RecA2 domain triggers changes in the position of the CTDs, which form a single entity with the RecA1 domain. Surprisingly, comparison of the four Prp2 structures revealed that the observed changes in the position of the CTDs are not related to the presence or absence of ADP (Fig. 3a).

\subsection{Variable $\beta$-hairpin conformation}

A superposition of the four compared Prp2 structures based on their RecA2 domains unveiled structural flexibility of the C-terminal domains and their coupled movement with the RecA1 domain. A closer look at the four superimposed RecA2 domains (Fig. 4a) shows substantial differences in the conformation of the $\beta$-hairpin (residues 596-610), resulting in a significant increase in the calculated r.m.s.d.s, which range from 0.71 to $2.15 \AA$ (average $1.61 \AA$ ) when compared with the r.m.s.d.s calculated between RecA2 domains without this flexible fragment $(0.50-0.86 \AA$, average $0.65 \AA)$. The observed conformational flexibility of the $\beta$-hairpin leads to substantial differences in the number of interdomain interactions formed between this structural element and mainly the winged-helix and OB-fold domains (Supplementary Table S1). The $\beta$-hairpins of NT-free and CF2 have similar conformations and protrude out of the molecular scaffold (Fig. 4a). The tips of these two $\beta$-hairpins were not resolved in the electron-density map and are probably disordered. In contrast, the $\beta$-hairpins of CF1 and CF3 are fully resolved and exhibit significantly different conformations and numbers of interdomain contacts (Supplementary Table S1). The largest number of interactions was observed for the deeply buried $\beta$-hairpin of $\mathrm{CF} 3$, which interacts mostly with the $\mathrm{C}$-terminal domains.

A thorough analysis of the structural superposition of the four Prp2 structures reveals an apparent correlation between the conformation of the $\beta$-hairpin and the position of the C-terminal domains with respect to the helicase core (Fig. 4a). Prp2 structures with the $\beta$-hairpin protruding out of the protein scaffold (NT-free and CF2) exhibit CTDs closer to the surface of the helicase core. Conversely, the structure with the buried $\beta$-hairpin (CF3) exhibits the most distant position of the CTDs relative to the RecA domains.

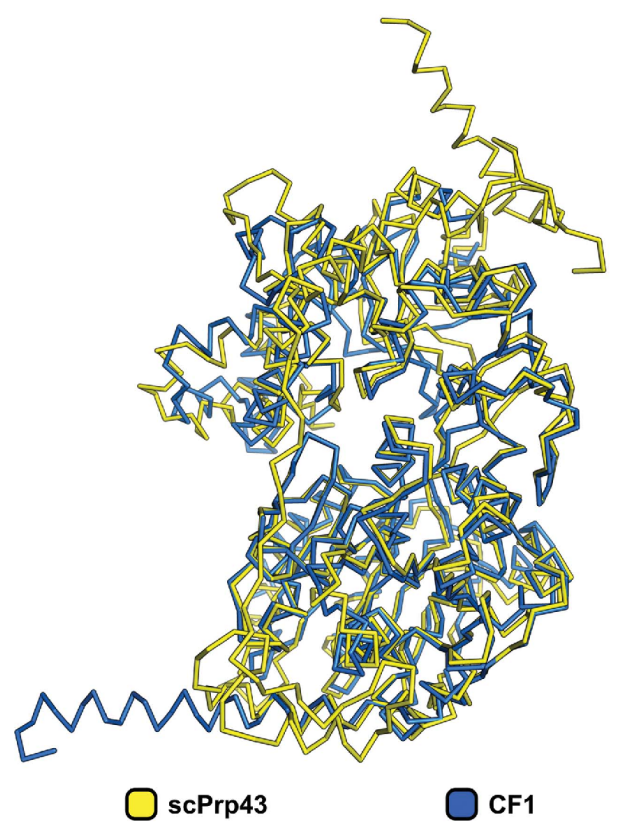

(a)

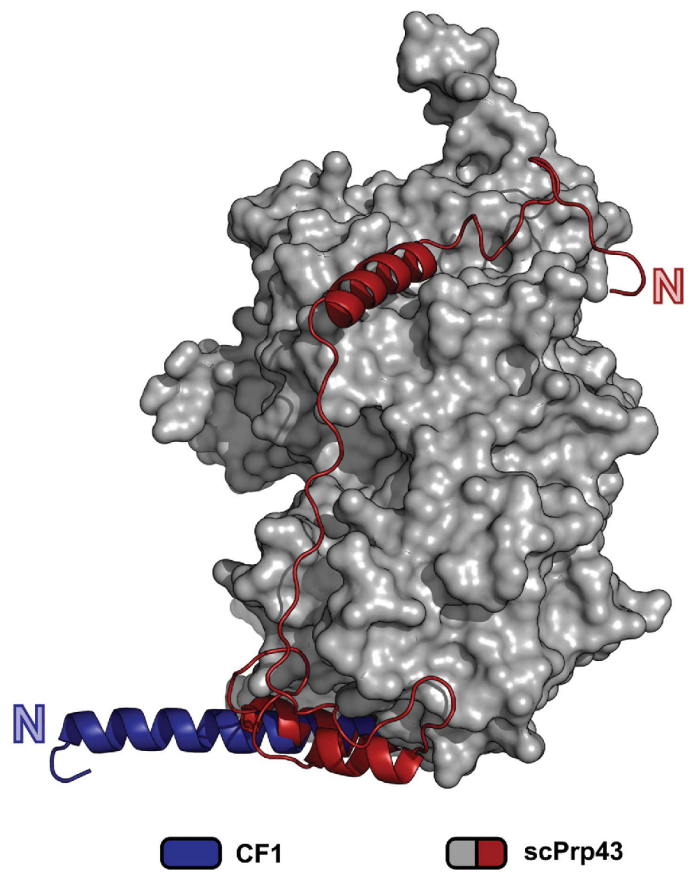

(b)

Figure 2

Structural comparison of ctPrp2 and scPrp43 (PDB entry 3kx2; He et al., 2010). (a) Superposition of ctPrp2 (blue) and scPrp43 (yellow), depicted here as ribbon models, reveals a high structural similarity, resulting in an r.m.s.d. of $1.3 \mathrm{~A}$ for 573 common $\mathrm{C}^{\alpha}$ atoms. $(b)$ The most prominent difference is found at the $\mathrm{N}$-termini of the proteins. The $\mathrm{N}$-termini of ctPrp2 and scPrp43 are shown as cartoons, whereas the protein core of scPrp43 is shown as a surface (grey). The N-terminal extension of ctPrp2 (blue) protrudes from the protein core, whereas the N-terminus of scPrp43 (red) wraps around the RecA1 domain and parts of the C-terminal domains. 


\section{Chapter 2: Crystal structure of the spliceosomal DEAH-box ATPase Prp2}

\section{research papers}

A comparable correlation can be observed in the structures of $C$. thermophilum Prp43. These structures exhibit significant conformational changes of the $\mathrm{C}$-terminal domains which are related to their individual functional states (Tauchert et al., 2016, 2017). It has been shown that the C-terminal domains are positioned closest to the helicase core in the ADP-bound state, are slightly more distant in the RNA/ADP- $\mathrm{BeF}_{3}$-bound state and are by far the furthest apart in the $\mathrm{ADP}-\mathrm{BeF}_{3}$-bound state. Although the degree of movement of the $\beta$-hairpin in these structures is decreased and the conformational divergence of the $\mathrm{C}$-terminal domains is much more pronounced when compared with the structures of Prp2 presented here, it is notable that the most distal $\beta$-hairpin is present in the ADPbound state, while the most integrated $\beta$-hairpins are found in the ADP-BeF ${ }_{3}$-bound state (Fig. $4 b$ ).

\subsection{Alternate ADP conformations}

The two RecA-like domains form a cleft harbouring the bound ADP nucleotide. In the reported crystal structures of ctPrp2 complexed with ADP, the nucleoside diphosphates are bound by Gly323, Gly325, Lys326, Thr327 and Thr328. An $\mathrm{Mg}^{2+}$ ion is coordinated by four water molecules, a $\beta$-phosphate $\mathrm{O}$ atom and the hydroxyl group of the Thr327 side chain.

Despite this similarity, the conformation of the adenosine and its binding mode differ significantly between $\mathrm{CF} 1 / \mathrm{CF} 3$ and CF2 (Fig. 5c). In CF1 and CF3 the adenine is sandwiched between Arg362 and Phe558 and the N6 atom forms a hydrogen bond to Ser358. The $3^{\prime}-\mathrm{OH}$ group of the ribose is involved in a polar interaction with Arg628. This conformation of the bound adenosine in the anti conformation in CF1/ CF3 is very similar to that observed in Prp43-ADP crystal structures (Tauchert et al., 2016; Walbott et al., 2010; He et al., 2010).

In $\mathrm{CF} 2$, a novel conformation of the adenosine has been observed which is unique among all known $\mathrm{DE} x \mathrm{D} / \mathrm{H}$-box helicase structures. While the conformation and binding mode of the diphosphate moiety remained unchanged, the base is flipped over into the syn conformation and points towards the RecA2 domain (Fig. 5d). In this new conformation (CF2) the adenine moiety forms two polar interactions with Asp582. The side chain of Arg362 is repositioned, now occupying the position of the adenine in $\mathrm{CF} 1 / \mathrm{CF} 3$, forms a hydrogen bond to the $3^{\prime}-\mathrm{OH}$ group of the ribose. Superposition of the two ADP molecules (CF1 and CF2) based on their ribose moieties clearly depicts the actual differences in conformations (Fig. $5 e)$. Although the sugar pucker remained unchanged $\left(\mathrm{C}^{\prime}\right.$ endo conformation), the torsion angles of the $\mathrm{C}^{\prime}-\mathrm{C}^{\prime}$ and $\mathrm{C}^{\prime}-\mathrm{O}^{\prime}$ bonds are changed in the CF2 conformation relative to those in $\mathrm{CF} 1 / \mathrm{CF} 3$ by 167 and $62^{\circ}$, respectively. The observed conformational heterogeneity of ADP in the reported Prp2 crystal structures (CF2 versus $\mathrm{CF} 1 / \mathrm{CF} 3$ ) reveals an intrinsic flexibility of the helicase core which is not related to large structural rearrangements or to crystal packing (CF3 and $\mathrm{CF} 2$ are isostructural).

Additionally, the novel binding mode of ADP is accompanied by a change in the conformation of a loop at the

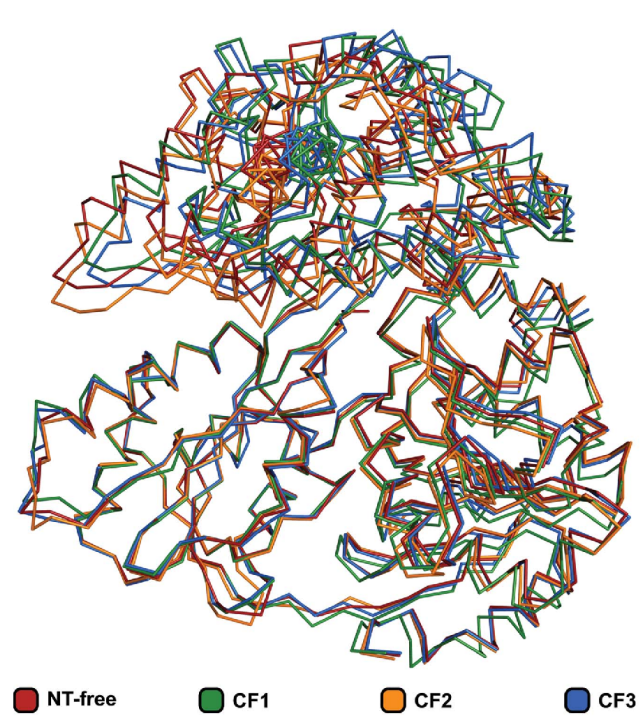

(a)

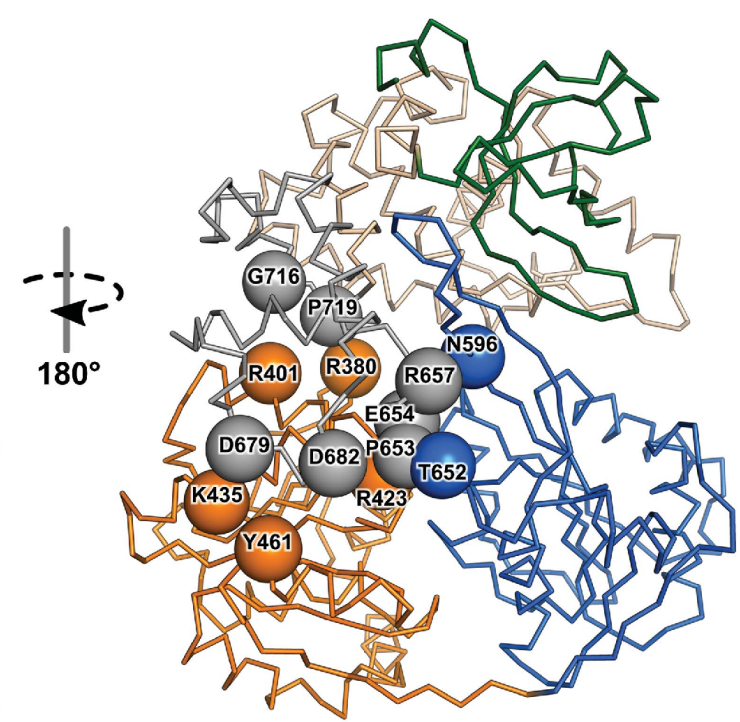

(b)

Figure 3

Flexibility of the C-terminal entity. (a) A superposition of all of the ctPrp2 structures via the RecA2 domain highlights the flexibility of the C-terminal domains with respect to the RecA2 domain. While the helicase cores superpose well, the C-terminal domains reveal various positions. $(b)$ Despite the observed flexibility, ten common interdomain interactions between the C-terminal domains and the helicase core can be found among the four structures. The residues involved in these conserved interactions are displayed as spheres and labelled accordingly. ctPrp 2 is shown as a ribbon model and the domains are coloured according to Fig. 1. 


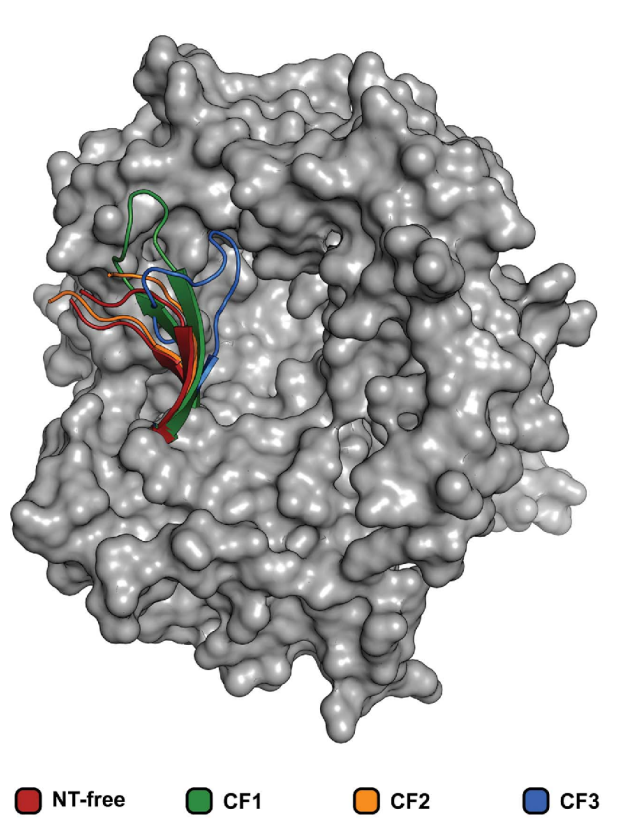

(a)

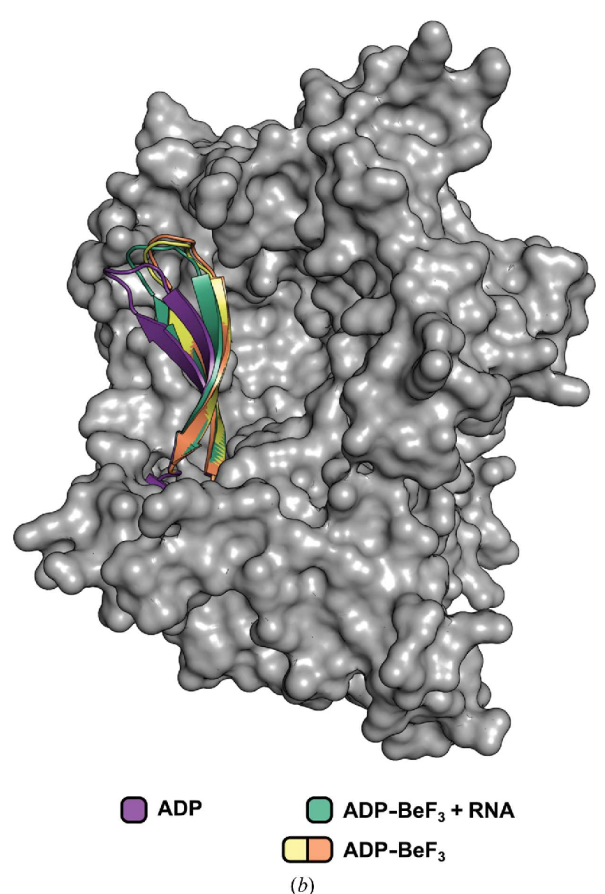

(b)

Figure 4

Comparison of the different $\beta$-hairpin conformations. (a) All ctPrp2 structures are superposed via their RecA2 domains. The different $\beta$-hairpins are presented as cartoon models using the colouring of Fig. 3(a), while the remaining part of the protein is shown as a surface (nucleotide-free structure only). The OB-fold domain was omitted for clarity. The superposition reveals that the $\beta$-hairpin of ctPrp2 can adopt different conformations. (b) Superposition of ctPrp43 structures in different functional states via their RecA2 domains (PDB entries 5d0u, 5lta, 5ltj and 5ltk; Tauchert et al., 2016, 2017). The structures are presented according to $(a)$ using different colours.

C-terminal end of the conserved sequence motif VI (Fig. $5 d$ ). A superposition of the RecA2 domains reveals that these loops in CF1 and CF3 adopt almost identical conformations and point towards ADP, whereas in CF2 this loop flips into a position more distant from the ADP. This alternate conformation of the loop enables the repositioning of the adenine as seen in CF2, as otherwise $\operatorname{Arg} 628$, which interacts with the ribose in $\mathrm{CF} 1$ and $\mathrm{CF} 3$, would hinder the novel conformation of ADP (Fig. 5c). Interestingly, this loop occupies a middle position in the nucleotide-free Prp2 structure when compared with the CF2 and CF1/CF3 Prp2-ADP structures.

\subsection{Nucleotide-free Prp2}

The nucleotide-free Prp2 contains a sulfate ion that occupies the position of the $\beta$-phosphate in the ADP-complex structures (Fig. 6). The $\mathrm{O}$ atoms of the sulfate ion form a similar network of interactions as the $\beta$-phosphates of the ADP-bound structures, namely with Gly323, Gly325, Lys 326 and Thr327. The overall structure of nucleotide-free Prp2 more closely resembles that of $\mathrm{CF} 2$ : the C-terminal domain is positioned close to the helicase core and the partially disordered $\beta$-hairpin protrudes out of the protein core (Fig. 3a). In contrast to CF1 and CF3, the motif VI loop is slightly flipped away from the ADP-binding pocket (Fig. $5 d$ ).
The nucleotide-free structure was the result of crystallization trials of Prp2 with the nonhydrolysable ATP analogue AMPPCP. In order to explain the lack of bound AMPPCP, binding studies of AMPPCP and ADP were conducted using isothermal titration calorimetry (ITC). This experiment resulted in no significant heat signal with the buffer conditions used when AMPPCP was titrated. Although the lack of thermal signal might also arise from enthalpy compensation in the case of a deprotonation upon binding, this result strongly suggests that Prp2 does not bind AMPPCP (Supplementary Fig. S1a). This finding is in agreement with the absence of AMPPCP in the nucleotide-free structure. The terminal $\mathrm{O}$ atom of the ADP in CF1-CF3 is involved in a hydrogen bond to Gly323 and this interaction could not take place with the methylene bridge of AMPPCP, which might explain the lack of binding of this ATP analogue. Binding studies via ITC were also performed for ADP as a control, and a dissociation constant $\left(K_{\mathrm{d}}\right)$ of $179 \mathrm{n} M$ was obtained (Supplementary Fig. $\mathrm{S} 1 b)$.

\section{Discussion}

Here, we report the first crystal structures of Prp2 in two different functional states (nucleotide-free and ADP-bound). The atomic models of both functional states share a very 


\section{Chapter 2: Crystal structure of the spliceosomal DEAH-box ATPase Prp2}

\section{research papers}

similar conformation and their overall structures strongly resemble the structure of the closely related DEAH-box helicase Prp43 (Fig. 2). However, despite these structural similarities, Prp2 and Prp43 differ significantly in their function. In contrast to all other spliceosomal DEAH-box proteins, no helicase activity has been observed for Prp2 in vitro

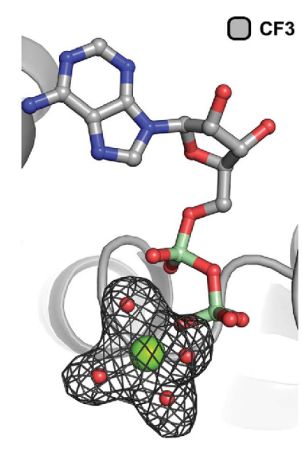

(a)

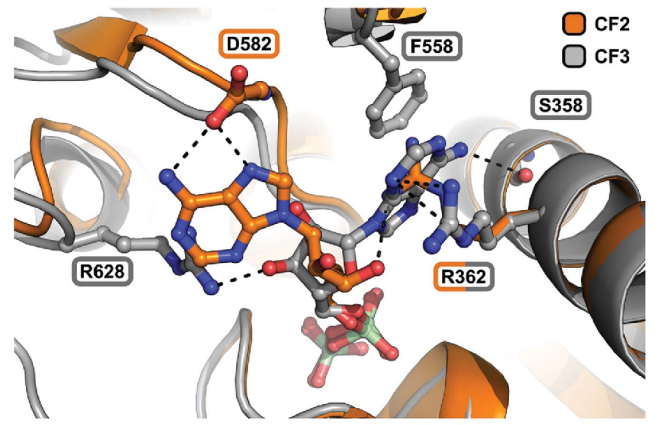

(d)

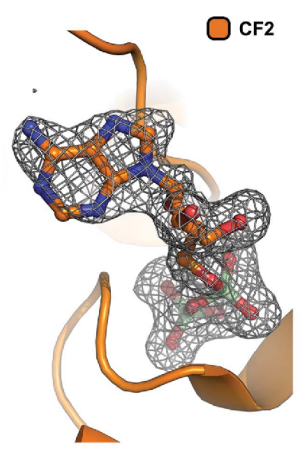

(b)

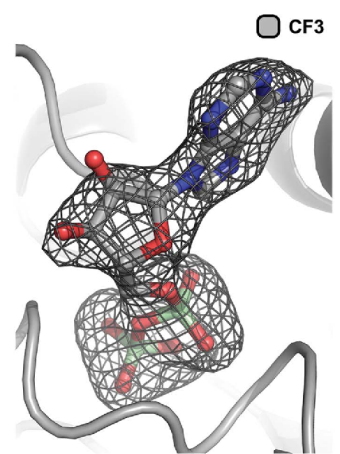

(c)

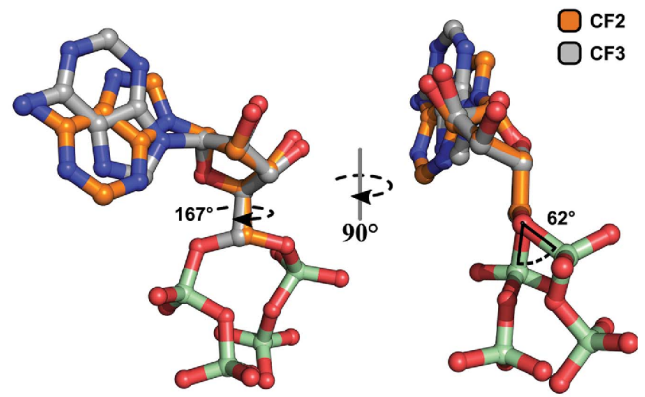

(e)

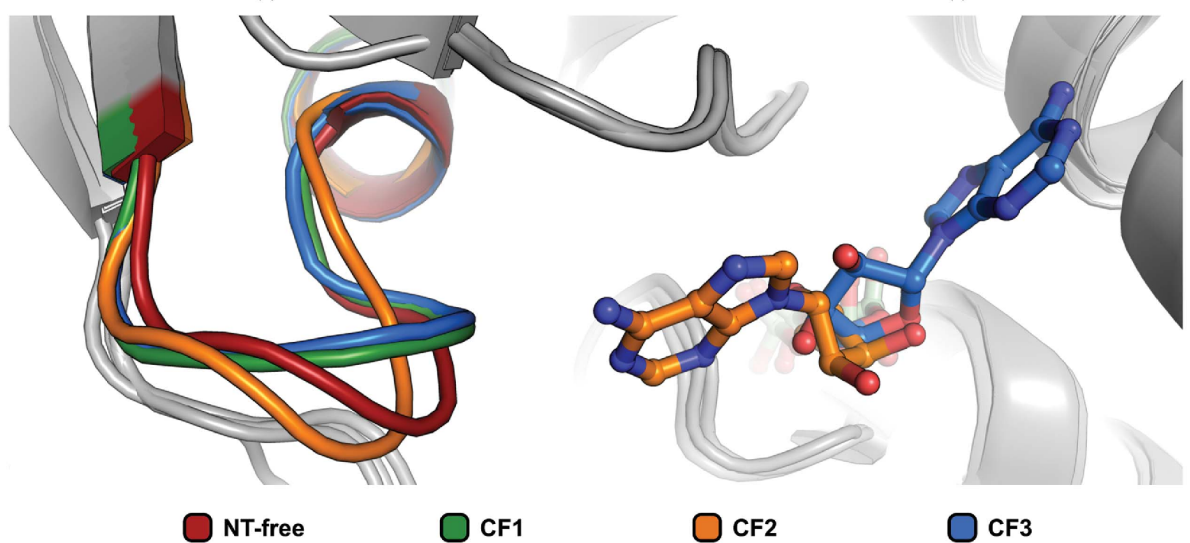

(f)

Figure 5

Comparison of differently bound ADP molecules. ADP molecules are presented as ball-and-stick models and the magnesium ion and water molecules as spheres. ctPrp2 is depicted as a cartoon representation with highlighted residues as ball-and-stick models. $(a)$ depicts the $m F_{\mathrm{o}}-D F_{\mathrm{c}}$ electron-density OMIT map for the magnesium ion with the four coordinated waters of CF3 (grey) contoured at $3 \sigma$. This OMIT map is representative of all ADP-bound structures. The OMIT maps at the same contour level for the ADP molecules of CF2 (orange) and CF3 are displayed in $(b)$ and $(c)$, respectively. $(d)$ On superposing the RecA1 domains of CF2 and CF3 the phosphate moieties align almost identically, but the adenosine is present in a previously undescribed conformation, leading to different adenosine interactions in CF2. (e) A superposition of the different ADP molecules via the ribose reveals that the adenine is present in a syn $(\mathrm{CF} 2)$ and an anti $(\mathrm{CF} 3)$ conformation and that the torsions along the $\mathrm{C}^{\prime}-\mathrm{C}^{\prime}$ bond and the $\mathrm{C}^{\prime}-\mathrm{O}^{\prime}$ bond are changed by 167 and $62^{\circ}$, respectively. $(f)$ Depending on the ADP conformations described in $(d)$ and $(e)$, a loop in the nearby motif VI adopts distinct conformations. A superposition of the RecA2 domains reveals a flipped conformation of this loop in the NT-free and CF2 structures compared with CF1 and $\mathrm{CF} 3$, which exhibit an ADP-binding pattern comparable to other DExD/H-box helicases. 


\section{Chapter 2: Crystal structure of the spliceosomal DEAH-box ATPase Prp2}

\section{research papers}

(Warkocki et al., 2015; Bao et al., 2017). This atypical lack of helicase activity appears to be consistent with the function of Prp2 as deduced from the recently determined cryo-EM structures of the $\mathrm{B}^{\text {act }}$ complex (Rauhut et al., 2016; Yan et al., 2016). Prp2 is located on the periphery of the $B^{\text {act }}$ spliceosome, contacting the outer side of the Hsh155 HEAT repeats, and therefore Prp2 is rather distant from the $5^{\prime}$ splice site and the branch-point adenosine (Supplementary Fig. S4). This is in agreement with previous biochemical data showing that Prp2 binds to the single-stranded intron $\sim 30$ nt downstream of the branch-point site (Liu \& Cheng, 2012; Warkocki et al., 2015). All data to date suggest that Prp2 does not act as a helicase by unwinding double-stranded RNAs, but rather functions as an RNA-dependent RNPase. The analysis of different functional states of Prp2 might provide insight into the peculiar manner of function of this spliceosomal DEAH-box helicase.

Structures of Prp2 in the nucleotide-free state and in the ADP-bound state are presented in this study. The latter is likely to represent the state of the DEAH-box protein after ATP hydrolysis and can thus be considered as a post-catalytic structural snapshot of this protein. Two of the three ADPbound Prp2 structures (CF1/CF3) not only show a high overall structural similarity to the known post-catalytic state of the DEAH-box helicase Prp43, but also exhibit a comparable ADP conformation (Fig. 2a). Interestingly, the third ADPbound structure (CF2) reveals a previously undescribed ADP conformation. In this structure the phosphates are bound in the same manner as in CF1/CF3, but the adenosine is flipped away from its position sandwiched between the RecA domains and points towards the RecA2 domain (Fig. 5d). This unique conformation is a consequence of changes in the torsion angles of the $\mathrm{C}^{\prime}-\mathrm{C}^{\prime}$ and $\mathrm{C}^{\prime}-\mathrm{O}^{\prime}$ bonds of 167 and $62^{\circ}$, respectively (Fig. 5e). Additionally, the adenine exhibits a syn conformation in $\mathrm{CF} 2$, in contrast to the more common anti conformation that is present in $\mathrm{CF} 1$ and $\mathrm{CF} 3$. The conformational changes of the ADP in CF2 seem to be accompanied by a flip in a neighbouring loop at the C-terminal end of motif VI and can only be sustained upon its conformational change, as otherwise the adenine would clash with Arg628 (Figs. 5d and $5 f$ ). Additionally, the more distal position of the loop slightly enlarges the entry to the nucleotide-binding pocket, which has also been observed in the nucleotide-free structure. The widening of the entry site to the binding cleft could play a role in the initial binding of ATP or the release of ADP, as it might make the binding pocket more easily accessible to the ATP molecule or promote the expulsion of ADP after completed hydrolysis. A further indication that the observed atypical ADP conformation might correspond to the ADPprerelease state can be based on an analysis of the interactions between Prp2 and the adenosine moiety. Compared with the typical conformation, two hydrogen bonds (Ser358-N6 and Arg628- $\left.3^{\prime}-\mathrm{OH}\right)$, a $\pi-\pi$ interaction and a cation $-\pi$ interaction are exchanged for three polar interactions (Asp582-N6/N7 and Arg362-3'-OH; Fig. 5d). The reduced number of interactions formed by the adenosine suggests a more loose association of the nucleotide with the helicase, which thereby also abandons its position in the interface between the two RecA domains. Thus, the Prp2 structure with the flipped ADP might correspond to a structural snapshot subsequent to the state observed in the other two ADP-bound states and prior to ADP release. The nucleotide-free structure could represent the state of Prp2 immediately after the release of ADP as well as shortly before the uptake of ATP. CF1 and CF3, on the other hand, display an ADP conformation that is already known from Prp43 structures, in which the adenine is sandwiched between an arginine from the RecA1 domain and a phenylalanine from the RecA2 domain (He et al., 2010; Walbott et al., 2010; Tauchert et al., 2016). These residues have been shown to be crucial for the NTPase and helicase activity of Prp43, in which the phenylalanine seems to play a role in coupling ATPase activity to helicase activity and an arginineto-alanine mutant impairs both functions (Robert-Paganin $e t$ al., 2017). The substantial role of these two residues in the catalytic processes of Prp43 suggests that the ADP-bound structures with the adenine sandwiched between these two amino acids might represent the state directly subsequent to catalysis.

Interestingly, no large structural rearrangements were observed between the structures of the nucleotide-free and ADP-bound states of Prp2, which share an almost unchanged nucleotide-binding pocket. The bound sulfate ion seems to mimic the $\beta$-phosphate of ADP (Fig. 6), as both groups share approximately the same position and an identical network of interactions. On the other hand, ADP and $\mathrm{P}_{\mathrm{i}}$ are generated after ATP hydrolysis, and to date no exact order of events after this step has been assigned for DEAH-box helicases. For the DEAD-box helicase Dbp5 it was demonstrated that release of $P_{i}$ is the rate-limiting step and is then followed by ADP release (Wong et al., 2016). Despite the high similarity of the helicase cores of these two families, DEAD-box helicases lack the C-terminal domains of the DEAH-box family, and the RecA domains can adopt so-called open and closed conformations, with the former resulting in a disrupted ATP-binding pocket (Ozgur et al., 2015). In contrast, the DEAH-box

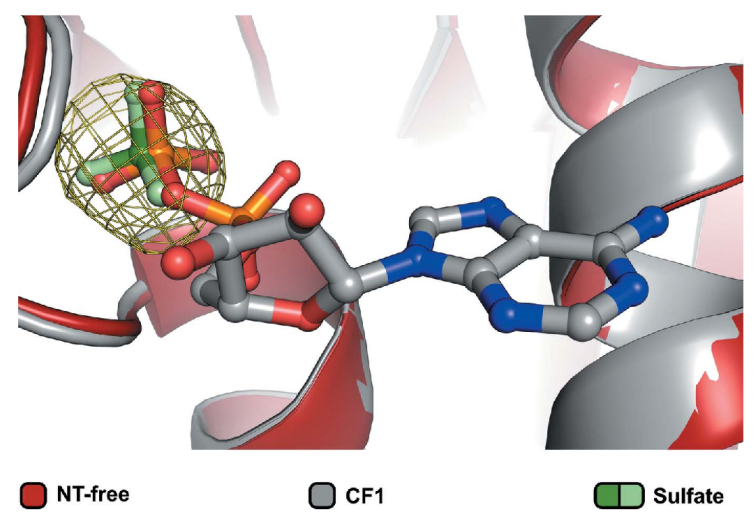

Figure 6

The nucleotide-free Prp2 structure with a bound sulfate molecule. A superposition of the RecA1 domains of the NT-free and CF1 structures shows that a sulfate that is present at the active site of the nucleotide-free structure adopts the same position as the $\beta$-phosphate of the bound ADP. 


\section{Chapter 2: Crystal structure of the spliceosomal DEAH-box ATPase Prp2}

\section{research papers}

helicases exhibit restricted movement of the RecA domains which results in the adoption of a permanent quasi-closed state with a preformed nucleotide-binding pocket. This difference in the mode of action might lead to different catalytic mechanisms and makes it difficult to predict whether helicases belonging to the DEAH-box and DEAD-box families act in the same manner. Since the RecA domains always stay in close proximity to each other in DEAH-box helicases, the nucleotide-binding site is completely blocked by the bound ADP molecule after catalysis. Thus, if $P_{i}$ is released before ADP a separate exit passage is necessary. Such an exit passage has not so far been identified in structures of this helicase family, and this makes it an interesting target for future studies. Since the nucleotide-free structure is likely to represent Prp2 after ADP release, the bound sulfate ion might mimic the remaining phosphate ion, which can now be released via the same passage as used for ADP exit/ATP entry. Although the reported structures might provide a hint to the sequence of events upon ATP hydrolysis, detailed biochemical analyses, as have been performed for the DEAD-box family, are required in order to completely understand the catalytic mechanism of DEAH-box proteins.

Recently, several crystal structures of Prp43 in the precatalytic state have been reported and have revealed how ATP analogues and RNA bind to DEAH-box helicases; they have also allowed the proposal of a sequence of events prior to ATP hydrolysis (Tauchert et al., 2017; He et al., 2017). In the proposed model, binding of ATP triggers a dramatic movement of the C-terminal domains relative to the RecA domains, leading to opening of the RNA-binding tunnel, which was previously completely enclosed. Once the $\mathrm{C}$-terminal domains are in a more distant position with regard to the helicase core, the RNA is then able to be incorporated into the RNAbinding tunnel, which closes again upon binding RNA, circumventing the need for a threading-like integration of the RNA. The varying number of contacts, especially between the $\mathrm{HB} / \mathrm{OB}$ and the helicase core, highlights the flexibility of the $\mathrm{C}$-terminal domains and is in accordance with this mechanism (Supplementary Table S1; Fig. 3a). However, on the opposite side of the RNA entrance the C-terminal domains need to be stably connected to the helicase core, and in Prp2 ten interdomain interactions were found that are present throughout all four structures (Fig. 3b, Supplementary Table S1). The WH domain is the only $\mathrm{C}$-terminal domain that is involved in these conserved interactions and thus represents the most stable anchor point. Considering the facts that these common interactions are also conserved in Prp43 and that the observed flexibility of the $\mathrm{C}$-terminal domains would support the current model of RNA binding, it seems likely that this mechanism might also apply to Prp2. Additionally, analysis of the Prp2 structures revealed a correlation between the position of the $\beta$-hairpin and the location of the $\mathrm{C}$-terminal domains. The structures with a protruding $\beta$-hairpin exhibit a conformation of the $\mathrm{C}$-terminal domains that is closer to the surface of the helicase core (Figs. $3 a$ and $4 a$ ). This correlation suggests a passive role of the $\beta$-hairpin to be unlikely as it would otherwise be coupled to the movement of the
C-terminal domains. Interestingly, a similar correlation can be found in the $C$. thermophilum Prp43 structures, although the conformational differences of the $\beta$-hairpins are smaller and the movement of the $\mathrm{C}$-terminal domains is more pronounced (Fig. 4b). Based on structural comparisons with the Ski2-like DNA helicase Hel308 it has been proposed that this $\beta$-hairpin might play a role in duplex melting, but since no detailed unwinding mechanism for DEAH-box members has been described to date, the exact purpose of this structural feature remains unclear (Büttner et al., 2007). The presented structures of Prp2 might link the repositioning of the $\beta$-hairpin to the movement of the $\mathrm{C}$-terminal domains, but either structures of DEAH-box proteins with dsRNA or in-depth biochemical studies are needed to shed light onto the function of the $\beta$-hairpin.

The N-terminal extension of Prp2 has been shown not to be essential for its function, and the constructs used almost completely lacked this domain (Edwalds-Gilbert et al., 2004). In contrast to the high sequence and structural similarity of the helicase core and C-terminal domains among DEAH-box proteins, the conservation of the N-terminal extensions is significantly lower and they have also been proposed to play a role in various noncatalytic functions such as regulation and recruitment (Supplementary Fig. S3; Tauchert et al., 2016; Wang \& Guthrie, 1998; Schneider \& Schwer, 2001). Although the major part of the N-terminal extension of Prp2 (269 of 296 amino acids) is missing in the constructs used for crystallization, a clear difference from the only structurally known genuine DEAH-box helicase Prp43 can already be found. The $\mathrm{N}$-terminal extension of Prp43 consists of three short $\alpha$-helices and wraps around the globular protein, whereas the part of the $\mathrm{N}$-terminal domain that is present in the Prp2 crystal structures shows a long $\alpha$-helix that protrudes out of the protein core (Fig. $2 b$ ). Although it is involved in crystal contacts with two symmetry-related molecules, secondary-structure predictions from numerous servers agree on the presence of an $\alpha$-helix between residues 270 and 296, as can be seen in CF1 (Supplementary Fig. S5; Kurowski \& Bujnicki, 2003). This protruding helix represents the last residues of the $\mathrm{N}$-terminal domain, which reaches out in order to make contacts with other spliceosomal factors distant from the main body of the helicase. This scenario is in agreement with cryo-EM data and cross-linking studies, revealing putative interaction sites of this $\mathrm{N}$-terminal extension with Prp45 (Prp2 Lys101/102/120/128 and Prp45 Lys274; Rauhut et al., 2016; Yan et al., 2016). However, owing to the high flexibility of these peripheral $\mathrm{B}^{\text {act }}$ spliceosome regions no detailed structural information for these interaction sites could be obtained to date. The distance between the beginning of the Prp2 N-terminal $\alpha$-helix and residue Ala292, which is positioned in the closest vicinity to the cross-linking site of Prp45, amounts to roughly $110 \AA$. This spatial separation can be bridged by an $\mathrm{N}$-terminal extension of at least 220 amino acids (220 in S. cerevisiae, 296 in $C$. thermophilum and 390 in H. sapiens), which is predicted to have a high $\alpha$-helical content. The last 26 residues of this domain, which are present in CF1, also adopt an $\alpha$-helical conformation. Interestingly, in human Prp2 (DHX16) the 


\section{Chapter 2: Crystal structure of the spliceosomal DEAH-box ATPase Prp2}

\section{research papers}

most N-terminal tip of the corresponding domain has been proposed to contain a PWI-like domain, which is also present in the spliceosomal helicases Brr2 (U5-200K) and has been predicted for Prp22 (DHX8) (Absmeier et al., 2015; Korneta $e t$ al., 2012). In Brr2 the PWI-like domain-containing N-terminal region has been proposed to act as a structural switch autoinhibiting Brr2 via substrate competition and conformational clamping (Absmeier et al., 2015; Agafonov et al., 2016; Nguyen et al., 2016). In the absence of detailed structural and biochemical information on the $\mathrm{N}$-terminal extension of Prp2, one can only speculate about the functional role of this domain. The presumably distant location from the catalytically active protein core seems to exclude it from catalysisrelated functions, with it rather being responsible for recruitment and proper positioning in the spliceosome.

Despite the many parallels between Prp2 and Prp43 discussed in this structural study, the key question of why Prp2 is not able to act as an actual helicase cannot be answered using the presented structures. Prp2 and Prp43 are known to interact with so-called G-patch proteins and the regulation of their functions is tightly linked to this interaction. Detailed biochemical as well as structural characterization of this interaction might help to understand the peculiar manner of function of Prp2. However, most importantly, structural insights into Prp2 in a precatalytic state with bound RNA and an ATP analogue might reveal differences from the existing precatalytic structures of Prp43 which could shed light onto the as yet unknown mechanism of action of Prp2.

\section{Related literature}

The following references are cited in the Supporting Information for this article: Fica et al. (2017) and Liu et al. (2017).

\section{Acknowledgements}

We thank HZB for the allocation of synchrotron-radiation beamtime leading to the NT-free and CF2 structures. The experiments for CF1 and CF3 were performed on beamline ID23-2 at the European Synchrotron Radiation Facility (ESRF), Grenoble, France. We are grateful to Max Nanao at the ESRF for providing assistance in using beamline ID23-2.

\section{Funding information}

This work was supported by the DFG, Sonderforschungsbereich 860 .

\section{References}

Absmeier, E., Rosenberger, L., Apelt, L., Becke, C., Santos, K. F., Stelzl, U. \& Wahl, M. C. (2015). Acta Cryst. D71, 762-771.

Adams, P. D. et al. (2010). Acta Cryst. D66, 213-221.

Agafonov, D. E., van Santen, M., Kastner, B., Dube, P., Will, C. L., Urlaub, H. \& Lührmann, R. (2016). RNA, 22, 1329-1337.

Amlacher, S., Sarges, P., Flemming, D., van Noort, V., Kunze, R., Devos, D. P., Arumugam, M., Bork, P. \& Hurt, E. (2011). Cell, 146, 277-289.

Bao, P., Hobartner, C., Hartmuth, K. \& Luhrmann, R. (2017). RNA, 23, 1770-1779.
Brautigam, C. A. (2015). Methods, 76, 124-136.

Büttner, K., Nehring, S. \& Hopfner, K. P. (2007). Nature Struct. Mol. Biol. 14, 647-652.

Chen, V. B., Arendall, W. B., Headd, J. J., Keedy, D. A., Immormino, R. M., Kapral, G. J., Murray, L. W., Richardson, J. S. \& Richardson, D. C. (2010). Acta Cryst. D66, 12-21.

Chiu, Y.-F., Liu, Y.-C., Chiang, T.-W., Yeh, T.-C., Tseng, C.-K., Wu, N.-Y. \& Cheng, S.-C. (2009). Mol. Cell. Biol. 29, 5671-5678.

Cordin, O., Banroques, J., Tanner, N. K. \& Linder, P. (2006). Gene, 367, 17-37.

Cordin, O. \& Beggs, J. D. (2013). RNA Biol. 10, 83-95.

Cordin, O., Hahn, D. \& Beggs, J. D. (2012). Curr. Opin. Cell Biol. 24, 431-438.

Ding, S. C. \& Pyle, A. M. (2012). Methods Enzymol. 511, 131-147.

Edwalds-Gilbert, G., Kim, D.-H., Silverman, E. \& Lin, R.-J. (2004). $R N A, \mathbf{1 0}, 210-220$

Emsley, P., Lohkamp, B., Scott, W. G. \& Cowtan, K. (2010). Acta Cryst. D66, 486-501.

Fairman-Williams, M. E., Guenther, U. P. \& Jankowsky, E. (2010). Curr. Opin. Struct. Biol. 20, 313-324.

Fica, S. M., Oubridge, C., Galej, W. P., Wilkinson, M. E., Bai, X.-C., Newman, A. J. \& Nagai, K. (2017). Nature (London), 542, 377380.

Ficner, R., Dickmanns, A. \& Neumann, P. (2017). Methods, 125, $63-$ 69.

He, Y., Andersen, G. R. \& Nielsen, K. H. (2010). EMBO Rep. 11, 180186.

He, Y., Staley, J. P., Andersen, G. R. \& Nielsen, K. H. (2017). RNA, 23, 1110-1124.

Hoskins, A. A. \& Moore, M. J. (2012). Trends Biochem. Sci. 37, 179188.

Houtman, J. C., Brown, P. H., Bowden, B., Yamaguchi, H., Appella, E., Samelson, L. E. \& Schuck, P. (2007). Protein Sci. 16, 30-42.

Kabsch, W. (2010). Acta Cryst. D66, 125-132.

Keller, S., Vargas, C., Zhao, H., Piszczek, G., Brautigam, C. A. \& Schuck, P. (2012). Anal. Chem. 84, 5066-5073.

Kim, S.-H. \& Lin, R.-J. (1996). Mol. Cell. Biol. 16, 6810-6819.

King, D. S. \& Beggs, J. D. (1990). Nucleic Acids Res. 18, 6559-6564.

Kleywegt, G. J. (1996). Acta Cryst. D52, 842-857.

Korneta, I., Magnus, M. \& Bujnicki, J. M. (2012). Nucleic Acids Res. 40, 7046-7065.

Krishnan, R., Blanco, M. R., Kahlscheuer, M. L., Abelson, J., Guthrie, C. \& Walter, N. G. (2013). Nature Struct. Mol. Biol. 20, 1450-1457. Kudlinzki, D., Schmitt, A., Christian, H. \& Ficner, R. (2012). Biol. Chem. 393, 1131-1140.

Kurowski, M. A. \& Bujnicki, J. M. (2003). Nucleic Acids Res. 31, 3305-3307.

Lardelli, R. M., Thompson, J. X., Yates, J. R. III \& Stevens, S. W. (2010). RNA, 16, 516-528.

Liu, H.-L. \& Cheng, S.-C. (2012). Mol. Cell. Biol. 32, 5056-5066.

Liu, S., Li, X., Zhang, L., Jiang, J., Hill, R. C., Cui, Y., Hansen, K. C., Zhou, Z. H. \& Zhao, R. (2017). Science, 358, 1278-1283.

Matera, A. G. \& Wang, Z. (2014). Nature Rev. Mol. Cell Biol. 15, 108121

McCoy, A. J., Grosse-Kunstleve, R. W., Adams, P. D., Winn, M. D., Storoni, L. C. \& Read, R. J. (2007). J. Appl. Cryst. 40, 658-674.

Mueller, U., Darowski, N., Fuchs, M. R., Förster, R., Hellmig, M., Paithankar, K. S., Pühringer, S., Steffien, M., Zocher, G. \& Weiss, M. S. (2012). J. Synchrotron Rad. 19, 442-449.

Murzin, A. G. (1993). EMBO J. 12, 861-867.

Nguyen, T. H. D., Galej, W. P., Bai, X.-C., Oubridge, C., Newman, A. J., Scheres, S. H. W. \& Nagai, K. (2016). Nature (London), 530, 298-302.

Ohrt, T., Prior, M., Dannenberg, J., Odenwälder, P., Dybkov, O., Rasche, N., Schmitzová, J., Gregor, I., Fabrizio, P., Enderlein, J. \& Lührmann, R. (2012). RNA, 18, 1244-1256.

Ozgur, S., Buchwald, G., Falk, S., Chakrabarti, S., Prabu, J. R. \& Conti, E. (2015). FEBS J. 282, 850-863. 


\section{Chapter 2: Crystal structure of the spliceosomal DEAH-box ATPase Prp2}

\section{research papers}

Prabu, J. R., Müller, M., Thomae, A. W., Schüssler, S., Bonneau, F. Becker, P. B. \& Conti, E. (2015). Mol. Cell, 60, 487-499.

Rauhut, R., Fabrizio, P., Dybkov, O., Hartmuth, K., Pena, V., Chari, A., Kumar, V., Lee, C.-T., Urlaub, H., Kastner, B., Stark, H. \& Lührmann, R. (2016). Science, 353, 1399-1405.

Richards, J. D., Johnson, K. A., Liu, H., McRobbie, A. M., McMahon, S., Oke, M., Carter, L., Naismith, J. H. \& White, M. F. (2008). J. Biol. Chem. 283, 5118-5126.

Robert-Paganin, J., Halladjian, M., Blaud, M., Lebaron, S., Delbos, L. Chardon, F., Capeyrou, R., Humbert, O., Henry, Y., Henras, A. K. Réty, S. \& Leulliot, N. (2017). Nucleic Acids Res. 45, 1539-1552.

Roy, J., Kim, K., Maddock, J. R., Anthony, J. G. \& Woolford, J. L. J (1995). RNA, 1, 375-390.

Schneider, S. \& Schwer, B. (2001). J. Biol. Chem. 276, 21184-21191. Silverman, E. J., Maeda, A., Wei, J., Smith, P., Beggs, J. D. \& Lin, R.-J. (2004). Mol. Cell. Biol. 24, 10101-10110.

Tauchert, M. J., Fourmann, J.-B., Christian, H., Lührmann, R. \& Ficner, R. (2016). Acta Cryst. F72, 112-120.

Tauchert, M. J., Fourmann, J. B., Luhrmann, R. \& Ficner, R. (2017) Elife, 6, e21510.
Wahl, M. C., Will, C. L. \& Lührmann, R. (2009). Cell, 136, 701-

Walbott, H., Mouffok, S., Capeyrou, R., Lebaron, S., Humbert, O., van Tilbeurgh, H., Henry, Y. \& Leulliot, N. (2010). EMBO J. 29, 2194-2204.

Wang, Y. \& Guthrie, C. (1998). RNA, 4, 1216-1229.

Warkocki, Z., Odenwälder, P., Schmitzová, J., Platzmann, F., Stark, H., Urlaub, H., Ficner, R., Fabrizio, P. \& Lührmann, R. (2009). Nature Struct. Mol. Biol. 16, 1237-1243.

Warkocki, Z., Schneider, C., Mozaffari-Jovin, S., Schmitzová, J. Höbartner, C., Fabrizio, P. \& Lührmann, R. (2015). Genes Dev. 29 , 94-107.

Will, C. L. \& Lührmann, R. (2011). Cold Spring Harb. Perspect. Biol. 3, a003707.

Wlodaver, A. M. \& Staley, J. P. (2014). RNA, 20, 282-294.

Wong, E. V., Cao, W., Vörös, J., Merchant, M., Modis, Y., Hackney, D. D., Montpetit, B. \& De La Cruz, E. M. (2016). J. Mol. Biol. 428, 492-508.

Yan, C., Wan, R., Bai, R., Huang, G. \& Shi, Y. (2016). Science, 353, 904-911. 
Chapter 2: Crystal structure of the spliceosomal DEAH-box ATPase Prp2

Volume 74 (2018)

Supporting information for article:

Crystal structure of the spliceosomal DEAH-box ATPase Prp2 Andreas Schmitt, Florian Hamann, Piotr Neumann and Ralf Ficner 
Chapter 2: Crystal structure of the spliceosomal DEAH-box ATPase Prp2

Acta Cryst. (2018). D74, doi:10.1107/S2059798318006356 Supporting information, sup-1

Table S1 Overview of interdomain interactions. Contacts between the helicase core and the $\mathrm{C}$-terminal domains are listed for each structure and residues are colorcoded according to Fig. 1. Salt bridges (SB) are highlighted with a cross.

\begin{tabular}{|c|c|c|c|c|}
\hline Apo & CF1 & CF2 & CF3 & \\
\hline 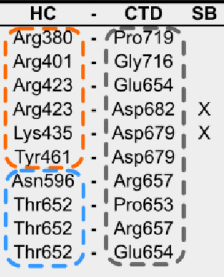 & 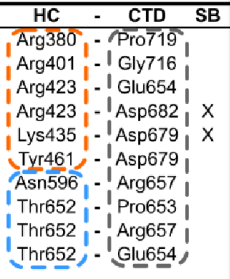 & 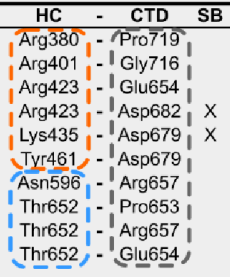 & 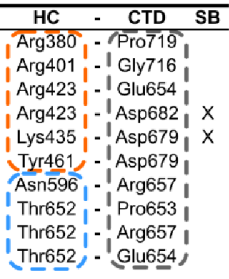 & $\begin{array}{l}\text { Conserved } \\
\text { Interdomain } \\
\text { Interactions }\end{array}$ \\
\hline 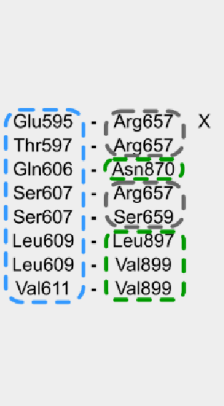 & 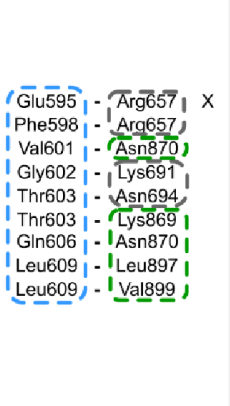 & 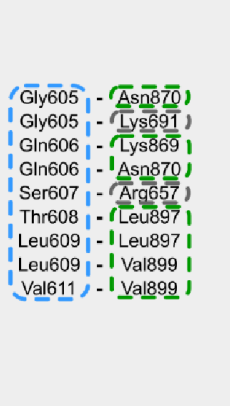 & 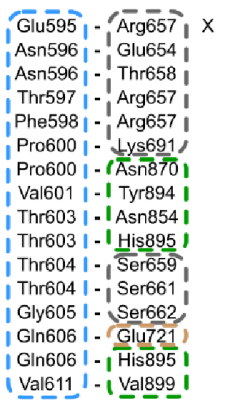 & $\begin{array}{c}\beta \text {-hairpin } \\
\text { Interactions }\end{array}$ \\
\hline 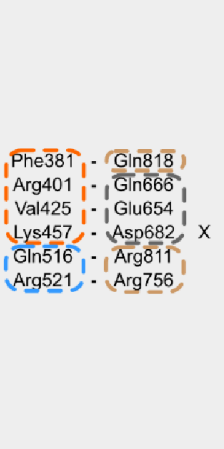 & 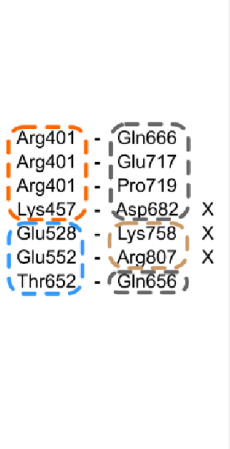 & 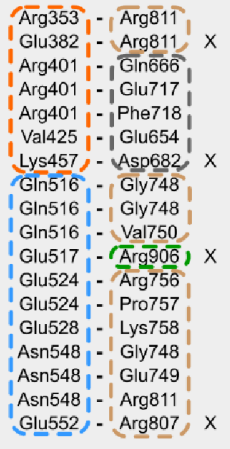 & 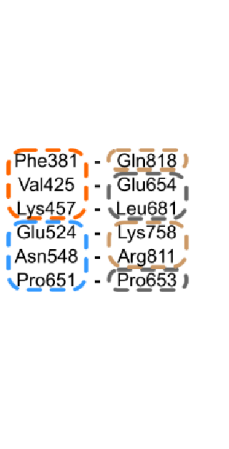 & $\begin{array}{l}\text { Remaining } \\
\text { Interactions }\end{array}$ \\
\hline 24 Contacts & 26 Contacts & 37 Cantacts & 32 Contacts & \\
\hline
\end{tabular}

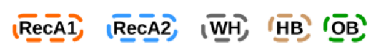

Helicase Core

(HC)

(CTD) 
Chapter 2: Crystal structure of the spliceosomal DEAH-box ATPase Prp2

a

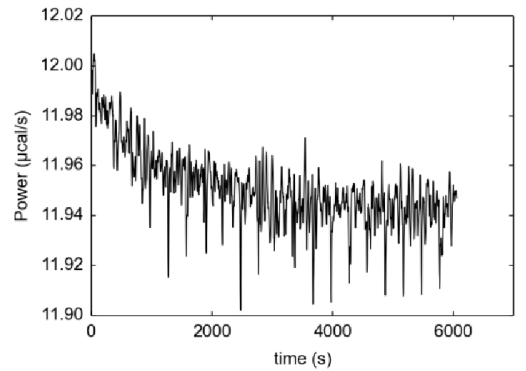

b

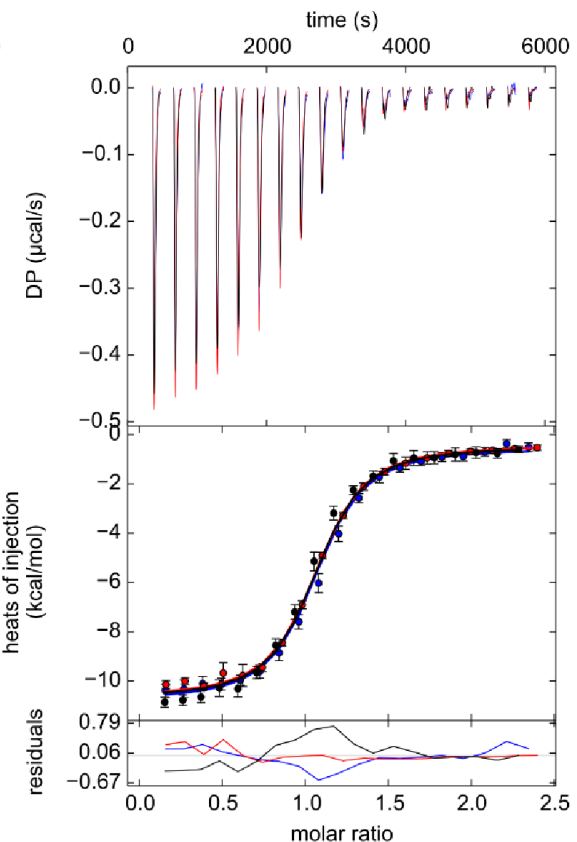

Figure S1 Isothermal titration calorimetry of Prp2 with AMPPCP and ADP. (a) The thermogram of the titration of AMPPCP to Prp2 did not result in thermic signals suited for integration, indicating there is no binding. (b) The binding of ADP was assessed and a dissociation constant $\left(\mathrm{K}_{\mathrm{d}}\right)$ of $179 \mathrm{nM}$ was determined $(\mathrm{n}=1.01, \Delta \mathrm{H}=-10.63 \mathrm{kcal} / \mathrm{mol}, \Delta \mathrm{S}=$ $\left.5.06 \mathrm{cal} / \mathrm{mol}^{*} \mathrm{~K}\right)$. Individual triplicates are depicted in red, blue and black. In the upper panel the thermograms are displayed, the middle panel shows the individual fittings with the correspondent residuals in the lower panel. 
Chapter 2: Crystal structure of the spliceosomal DEAH-box ATPase Prp2
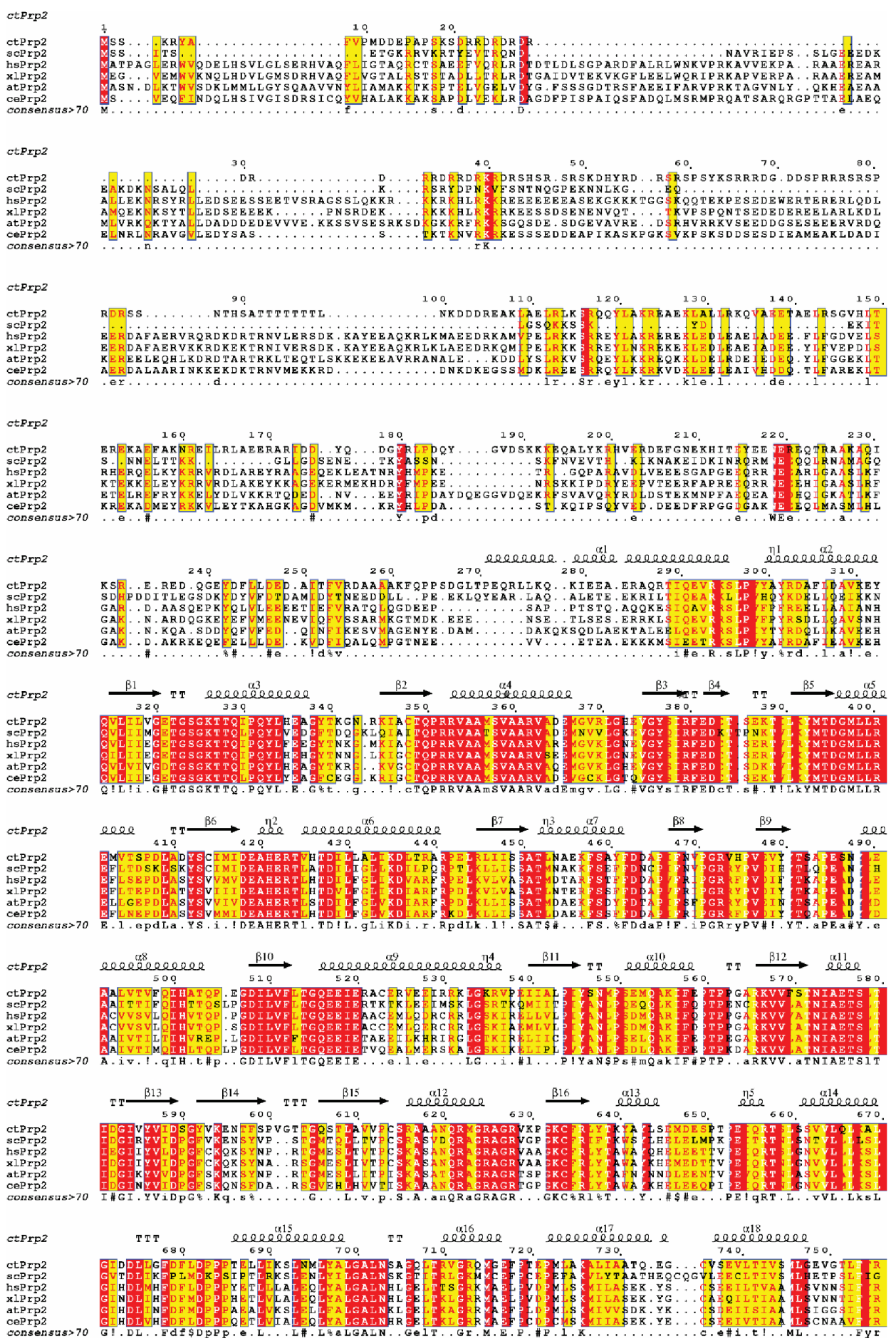
Chapter 2: Crystal structure of the spliceosomal DEAH-box ATPase Prp2
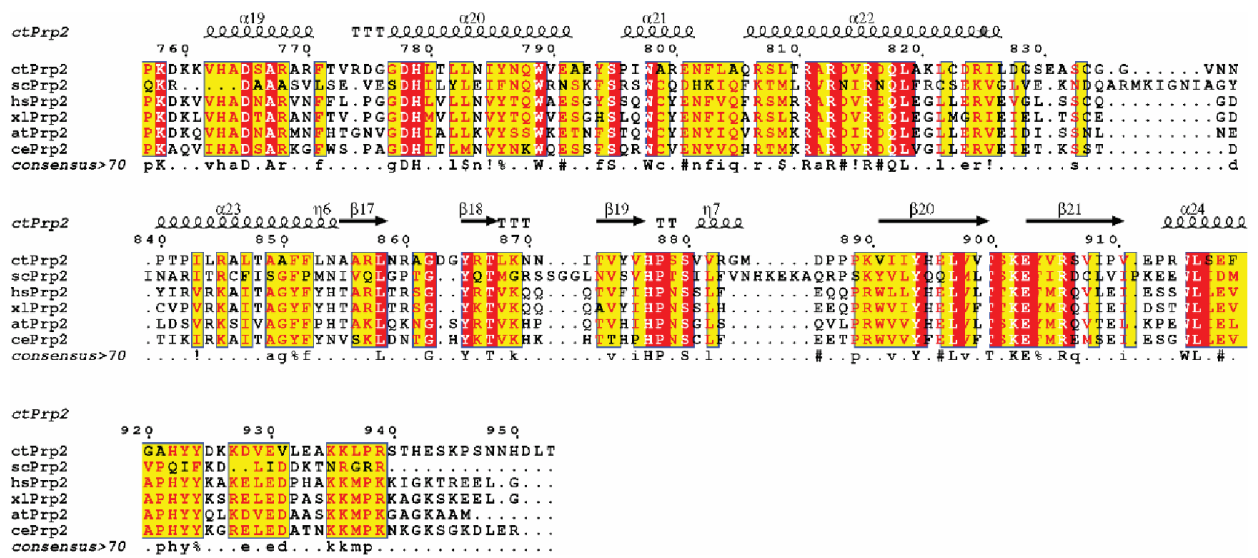

Figure S2 Sequence alignment of Prp2 from Chaetomium thermophilum (ct),

Saccharomyces cerevisiae (sc), Homo sapiens ( $h$ s), Xenopus laevis (xl), Arabidopsis thaliana (at) and Caenorhabditis elegans (ce). Secondary structure elements from CF2 are displayed on top of the corresponding sequence. 
Chapter 2: Crystal structure of the spliceosomal DEAH-box ATPase Prp2

Acta Cryst. (2018). D74, doi:10.1107/S2059798318006356 Supporting information, sup-5
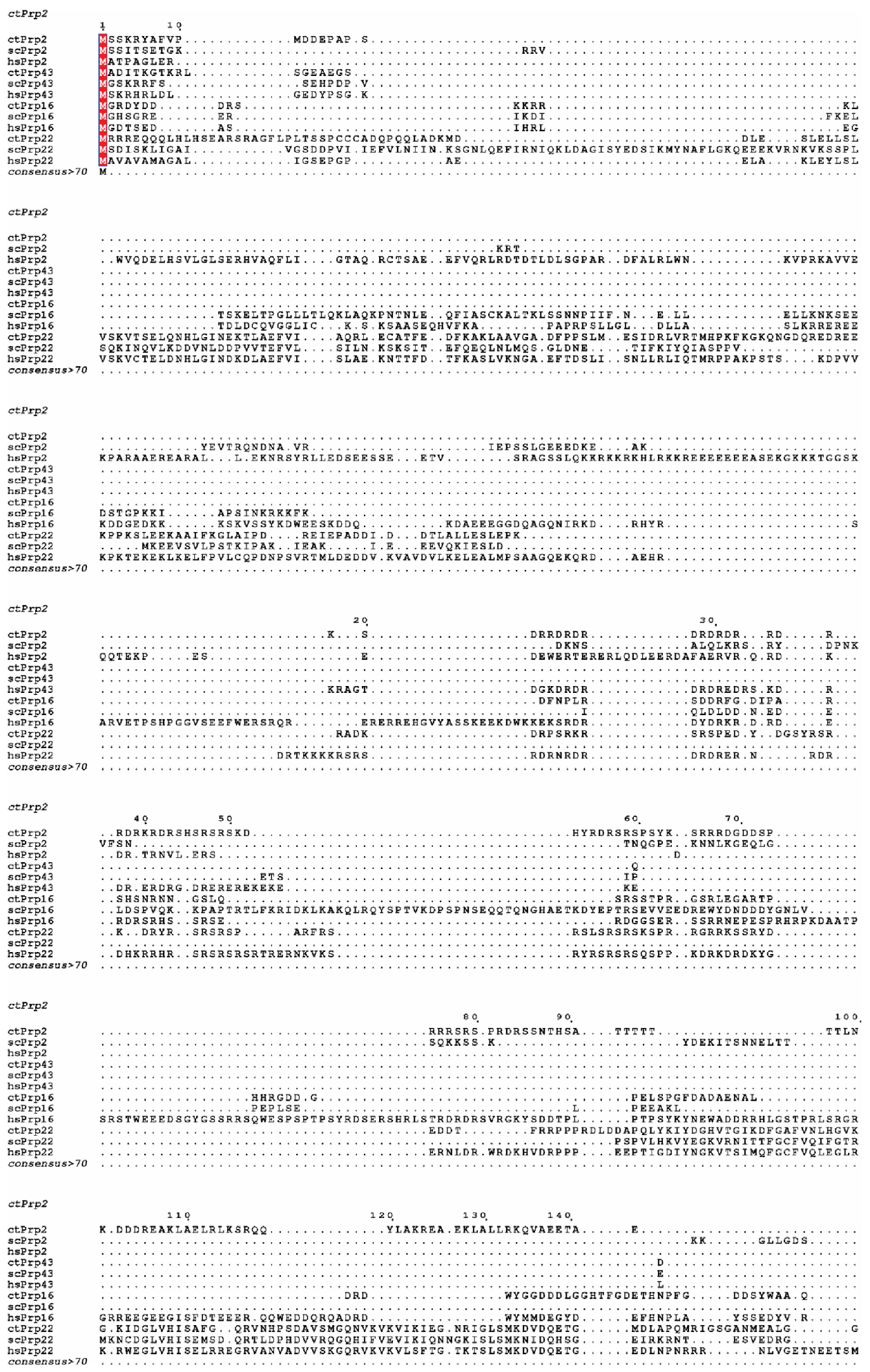
Chapter 2: Crystal structure of the spliceosomal DEAH-box ATPase Prp2

Acta Cryst. (2018). D74, doi:10.1107/S2059798318006356 Supporting information, sup-6

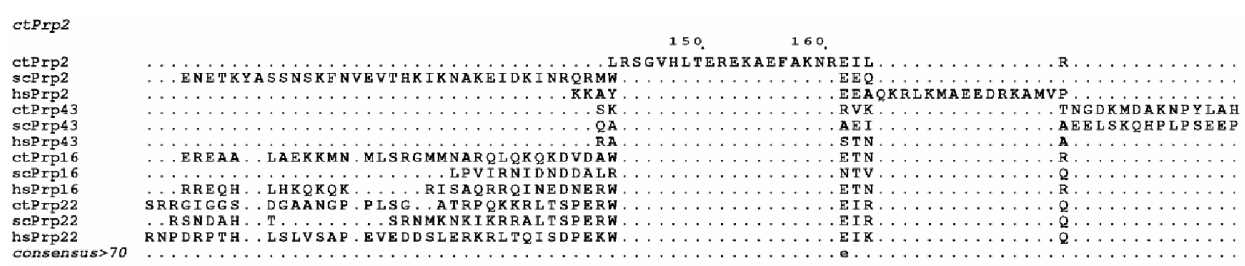

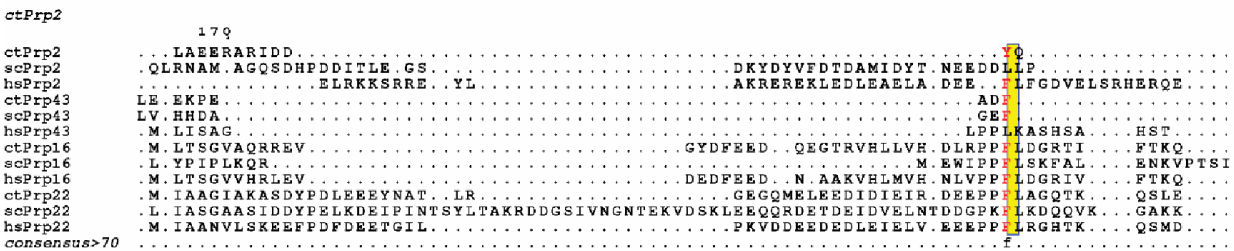

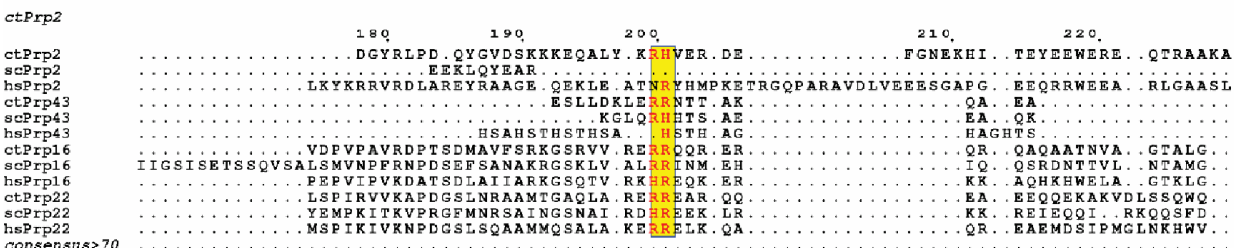

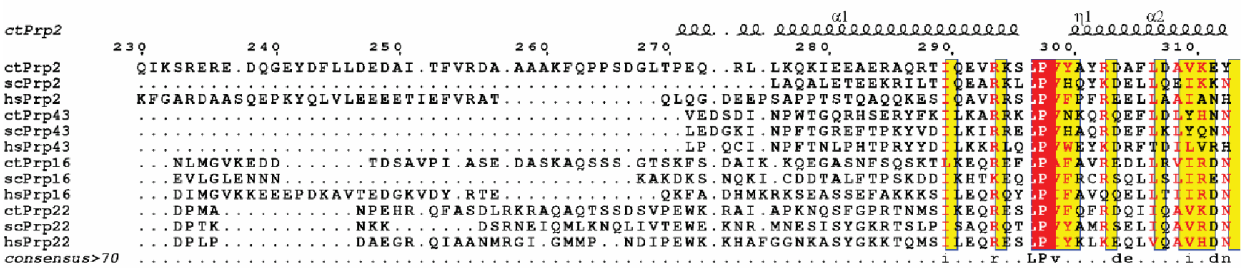
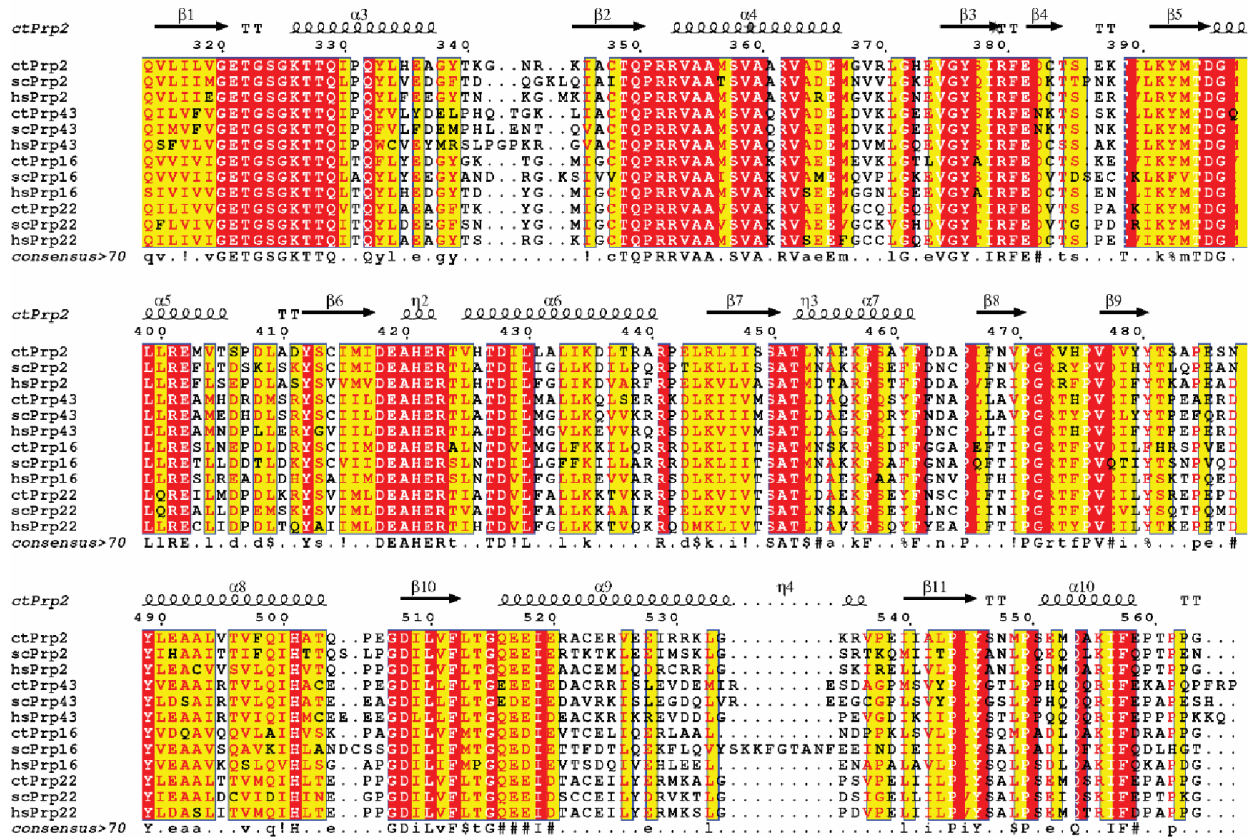

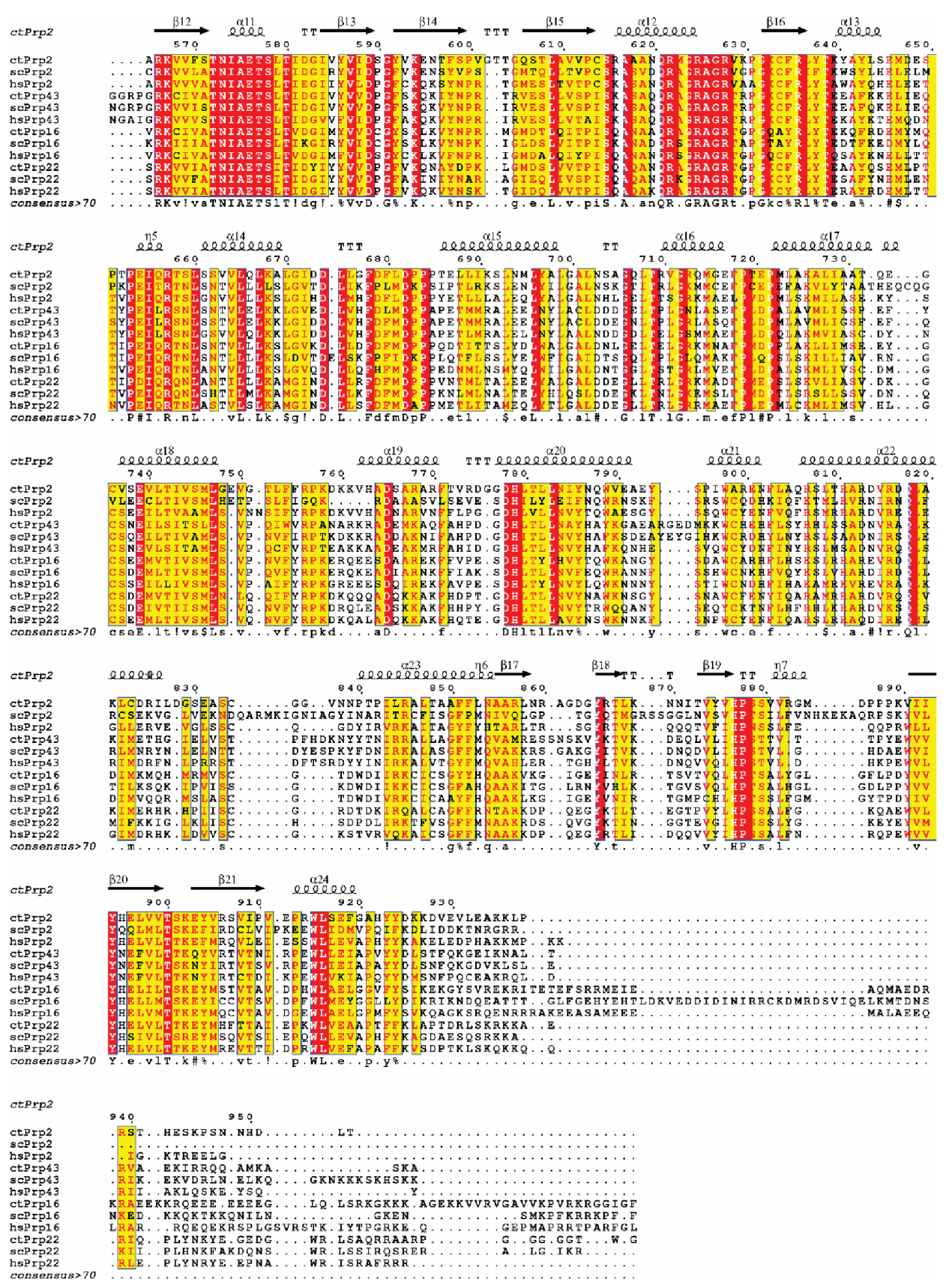

Figure S3 Sequence alignment of Prp2, Prp43, Prp16 and Prp22 from Chaetomium thermophilum (ct), Saccharomyces cerevisiae (sc) and Homo sapiens ( $h s$ ). Secondary structure elements from CF2 are displayed on top of the corresponding sequence. 

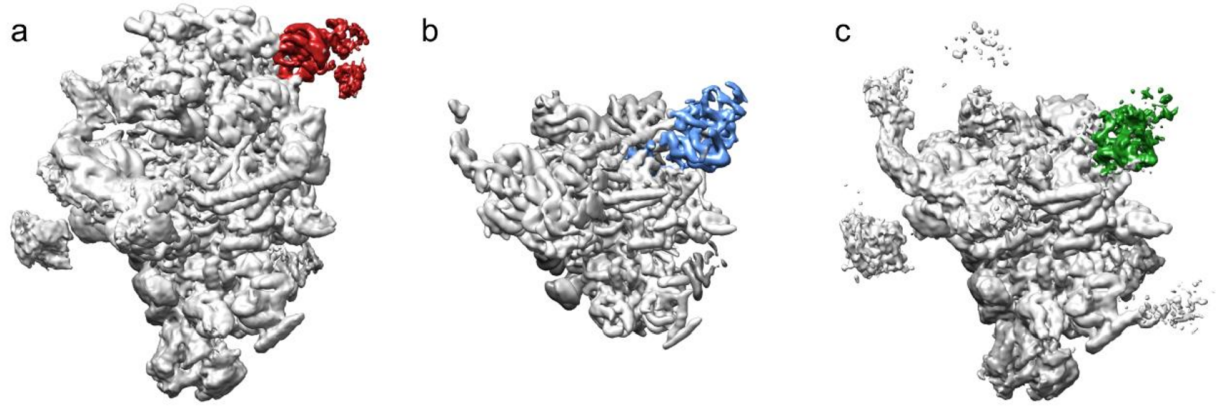

Figure S4 Position of DEAH-box helicases in the spliceosome. The cryo-EM maps of the Saccharomyces cerevisiae $\mathrm{B}^{\text {act }}$ complex (a, Yan et al., 2016), Saccharomyces cerevisiae postcatalytic P complex (b, Liu et al., 2017) and Saccharomyces cerevisiae spliceosome complex remodelled for exon ligation (c, Fica et al., 2017) superimposed and are shown in grey and DEAH-box helicases of each complex are depicted in red (Prp2), blue (Prp22) and green (Prp16), respectively. All DEAH-box helicases are found in the periphery of their respective spliceosomal complex.

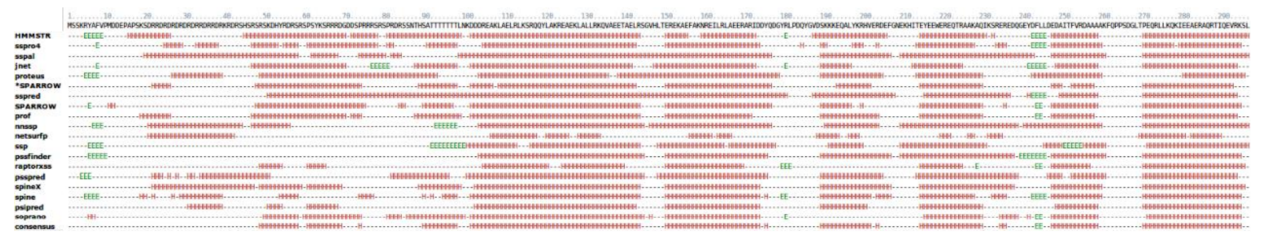

Figure S5 Secondary structure prediction of N-terminal extension of Chaetomium thermophilum Prp2. The secondary structure prediction was performed using the GeneSilico metaserver (Kurowski \& Bujnicki, 2003). Used prediction server for the metasearch are stated the left followed by the corresponding prediction on the right. Residues predicted to be part of a helical secondary structure are highlighted by " $\mathrm{H}$ " whereas residues predicted to be part of beta strands are displayed as "E". 


\section{Chapter 3: Structural analysis of the interaction of Spp2 G-patch with Prp2}

This manuscript was submitted to PNAS and is currently under review.

\section{Structural analysis of the intrinsically disordered splicing factor Spp2 and its binding to the DEAH-box ATPase Prp2}

Florian Hamann ${ }^{\S 1}$,Andreas Schmitt $^{\S 1}$, Filippo Favretto $^{\S 2}$, Romina Hofele ${ }^{3}$, Piotr Neumann ${ }^{1}$, ShengQi Xiang², Henning Urlaubb,4, Markus Zweckstetter ${ }^{2,5^{*}}$ and Ralf Ficner $^{1 *}$

${ }^{1}$ Department of Molecular Structural Biology, Institute for Microbiology and Genetics, GZMB, GeorgAugust-University Göttingen, Justus-von-Liebig-Weg 11, 37077 Göttingen, Germany

${ }^{2}$ Department for NMR-Based Structural Biology, Max Planck Institute for Biophysical Chemistry, 37077 Göttingen, Germany

3 Bioanalytical Mass Spectrometry Group, Max Planck Institute for Biophysical Chemistry, 37077 Göttingen, Germany

${ }^{4}$ Bioanalytics, Department of Clinical Chemistry, University Medical Center Göttingen, 37075 Göttingen, Germany

${ }^{5}$ German Center for Neurodegenerative Diseases (DZNE), Göttingen, Germany

${ }^{\S}$ These authors contributed equally.

* To whom correspondence should be addressed. Tel: +49 551 3914072; Fax: +49 551 3914082; Email: rficner@uni-goettingen.de; mazw@nmr.mpibpc.mpg.de 
Chapter 3: Structural analysis of the interaction of Spp2 G-patch with Prp2

\section{Author contributions:}

F.H.: Experimental design, molecular cloning, protein expression and purification, crystallization, data collection and processing, structure determination, structure refinement and analysis, Isothermal titration calorimetry (ITC) measurements, data analysis, preparation of figures and tables, writing - original draft, writing - review and editing

A.S.: Experimental design, molecular cloning, protein expression and purification, crystallization, data collection and processing, structure determination, structure refinement, circular dichroism measurements, secondary structure prediction

F.F.: NMR measurements and analysis, preparation of figure

R.H.: cross-linking studies and analysis

P.N.: Data collection and processing, structure determination, structure refinement, writing - review and editing

M.Z.: Experimental design, NMR analysis, writing - editing and review

R.F.: Experimental design, crystal structure analysis, data analysis, writing - review and editing, supervision, project leader 
Chapter 3: Structural analysis of the interaction of Spp2 G-patch with Prp2

\begin{abstract}
The spliceosome consists of five small RNAs and more than 100 proteins. Almost 50\% of the human spliceosomal proteins were predicted to be intrinsically disordered or to contain disordered regions, among them the G-patch protein Spp2. The G-patch region of Spp2 binds to the DEAH-box ATPase Prp2, and both proteins together are essential for promoting the transition from the $\mathrm{B}^{\text {act }}$ to the catalytically active $\mathrm{B}^{*}$ spliceosome. Here we show by CD and NMR spectroscopy that Spp2 is intrinsically disordered in solution. Crystal structures of a complex consisting of Prp2-ADP and the G-patch domain of Spp2 demonstrate that the G-patch gains a secondary structure element when it is bound to Prp2. While the N-terminal region of the G-patch always folds into an a-helix in five different crystal structures, the C-terminal part is able to adopt two alternative conformations. NMR studies further revealed that the N-terminal part of the Spp2 G-patch, which is the most conserved region in different G-patch proteins, transiently samples helical conformations, possibly facilitating a conformational selection binding mechanism. The structural analysis unveils the role of conserved residues of the G-patch in the dynamic interaction mode of Spp2 with Prp2, which is vital to maintain the binding during domain movements needed for RNA translocation by Prp2.
\end{abstract}

\title{
Significance statement
}

The G-patch domain is found in eukaryotic and viral proteins involved in protein-protein and protein-nucleic acid interactions. Some G-patch proteins play a vital role in the stimulation of the DEAH-box ATPases Prp2 and Prp43, however, their structural characterization and the exact binding mode have remained elusive. By studying the interaction of the Spp2 G-patch domain with Prp2 by means of X-ray crystallography and NMR, we could show that the G-patch is mostly disordered in solution but adopts a defined secondary structure element upon binding to Prp2. While one interaction site serves as a stable anchor for the binding, the other site exhibits a flexible interaction mode that is needed to adapt to the different conformations of Prp2. 
Chapter 3: Structural analysis of the interaction of Spp2 G-patch with Prp2

\subsection{Introduction}

Intrinsically disordered proteins (IDPs) or protein regions (IDRs) lack stable secondary and tertiary structure under physiological conditions and in the absence of an interaction partner or ligand. However, IDPs and IDRs might undergo a disorder-to-order transition upon binding to their targets, which has been referred to as “coupled folding and binding” or "induced folding”. The disorder of proteins provides functional advantages like the adaptability to different binding partners or the ability to bind with high specificity but relatively low affinity, which enables fast association or dissociation of the IDPs/IDRs to dynamic complexes (1-3).

IDPs and IDRs are widespread throughout the family of proteins involved in pre-mRNA splicing, as it has been predicted that about $45 \%$ of the human spliceosomal proteins are fully disordered and about $80 \%$ of the spliceosomal proteins contain disordered regions with a length of at least 30 residues (4). The spliceosome is a highly dynamic multi-megadalton ribonucleoprotein (RNP) complex, which catalyzes the removal of noncoding introns from precursor messenger RNAs (pre-mRNAs) in the nucleus of eukaryotic cells (5-7). The spliceosomes of all organisms consist of five uracil-rich small nuclear RNAs (UsnRNAs), a defined set of proteins stably associated with these UsnRNAs forming the UsnRNPs, and a large number of additional spliceosomal proteins, which might only transiently bind to the spliceosome (8). Notably, the total number of spliceosomal proteins varies significantly with the organism (9).

For each intron to be excised, the spliceosome assembles newly onto the pre-mRNA in a stepwise order. Initially the U1 snRNP binds to the 5' splice site of the pre-mRNA and the U2 snRNP to the branch point sequence (BPS), resulting in formation of the spliceosomal A complex. Subsequently, the U4/U6.U5 trisnRNP joins the spliceosome, leading to the catalytically inactive $\mathrm{B}^{\text {act }}$ complex. In the subsequent step, the catalytically active $\mathrm{B}^{*}$ complex is formed, which catalyzes the first transesterification reaction. After that, further remodeling leads to the formation of complex $\mathrm{C}$ and the second transesterification reaction takes place. Finally, the spliceosome is disassembled resulting in the release of the spliced mRNA and the intron lariat.

During assembly, splicing reaction and disassembly, the spliceosome undergoes large compositional and conformational changes including the remodeling of RNA-RNA, RNA-protein and protein-protein interactions (8). These rearrangements are mainly driven by eight DExD/H-box ATPases which use the energy from ATP hydrolysis to unwind dsRNA and/or to remodel RNA-protein interactions (10-12). These ATPases belong to the helicase superfamily 2 (SF2) and share a common fold consisting of two RecA-like domains which form the helicase core (13). Additional domains located C-terminal of the helicase core have been shown to act as binding platform for interaction partners or have a regulatory effect on the ATPase activity of the helicase $(14,15)$. The transition into the catalytically active $\mathrm{B}^{*}$ complex is promoted by the 
Chapter 3: Structural analysis of the interaction of Spp2 G-patch with Prp2

DEAH-box ATPase Prp2 together with its cofactor Spp2 (16-19). The Prp2-specific cofactor Spp2 belongs to the family of G-patch containing proteins. The name-giving glycine-rich patch (G-patch) consists of at least six conserved glycines and was first identified in RNA-associated proteins by bioinformatic analysis (20). G-patch proteins are absent in archaea and bacteria but present in some retroviral proteins and widespread among eukaryotes, where the G-patch domain can be found in various RNA and DNA-binding proteins (21). The G-patch protein Ntr1 strongly enhances the ATPase and helicase activities of the spliceosomal DEAH-box helicase Prp43 (22, 23). This stands in contrast to Spp2, which only stimulates the RNA-dependent ATPase activity of isolated Prp2, but not any helicase activity (24). Actually, no helicase activity could be observed for isolated Prp2 or the Prp2-Spp2 complex within the spliceosome $(25,26)$. This appears to be consistent with the recently determined cryo-EM structures of the $\mathrm{B}^{\text {act }}$ complex, as Prp2 binds just to single stranded RNA at the periphery of the spliceosome $(27,28)$. These cryo-EM structures, however, do not provide any information regarding Spp2. In addition to the cryo-EM structures, biophysical studies on spliceosomal DEAH-box ATPases Prp16 and Prp22 also argue in favor of translocation along a singlestranded RNA being the primary function of these proteins rather than RNA duplex unwinding (29). Recent structural studies have shown on a molecular level that RNA translocation is a highly dynamic process that requires DEAH-box ATPases to toggle between open and closed conformations of the helicase core (30, 31). Here, the motif $\mathrm{V}$ within the RecA2 domain senses the bound nucleotide and ensures the proper positioning of this domain in accordance to the catalytic state.

In order to gain insights into the structure of Spp2 and to understand how its interaction to Prp2 could be maintained despite the pronounced domain movements needed during RNA translocation, we examined unbound Spp2 in solution by means of CD and NMR spectroscopy, and determined five crystal structures of Prp2 in complex with the G-patch domain of Spp2 (Spp2 $\left.{ }_{G-p a t c h}\right)$. Spp2 ${ }_{G-p a t c h}$ is unfolded in solution and only transiently samples an $\alpha$-helical conformation in its $\mathrm{N}$-terminal part. This N-terminal amphipathic helix stably binds Prp2 mainly via hydrophobic interactions with a conserved hydrophobic patch at the wingedhelix domain (WH). While this part of the G-patch is indifferent in all determined crystal structures, the Cterminal part binds to the RecA2 domain and adopts two alternative conformations. Although the binding of Spp2 $2_{\text {G-patch }}$ does not show major structural effects on Prp2 when compared to structures of Prp2 alone, the crystallographic snapshots of the interaction demonstrate that the N-terminal part of the G-patch serves as a stable anchor point whereas the remaining regions associate in a much more flexible manner that likely guarantees its attachment to the mobile RecA2 domain. 
Chapter 3: Structural analysis of the interaction of Spp2 G-patch with Prp2

\subsection{Results}

\subsubsection{Purified scSpp2 is intrinsically disordered}

The G-patch protein Spp2 from S. cerevisiae (scSpp2) consists of 185 amino acids and is predicted to be intrinsically disordered (Figure 1A), however, experimental data regarding the folding and oligomerization state of scSpp2 has not been available (32). Since expression of full length scSpp2 in E. coli was unsuccessful, truncated scSpp2 $2_{10-185}$ lacking the N-terminal nine residues was used for further experiments. Size exclusion chromatography of scSpp $2_{10-185}$ resulted in an apparent molecular mass of about $65 \mathrm{kDa}$, which is about three times higher than the calculated molecular mass of scSpp2 ${ }_{10-185}$. In contrast, multi angle light scattering (MALS) showed that this truncated Spp2 is a monomer, indicating that the protein behaves like a partially or fully unfolded protein (Fig. S1). In addition, circular dichroism (CD) measurements were performed with scSpp $2_{10-150}$ as well as with the further truncated version scSpp $2_{100-150}$ containing only the Gpatch region. The CD spectra clearly show the absence of stable secondary structure elements for both samples (Figure 1B). To study the properties of scSpp2 in more detail, a heteronuclear singular quantum coherence (HSQC) NMR spectrum of ${ }^{15} \mathrm{~N}$ labeled scSpp2 ${ }_{10-185}$ was recorded (Figure 1C). The NMR spectrum was of high quality with a narrow distribution of the cross peaks around $8 \mathrm{ppm}$ of the ${ }^{1} \mathrm{H}$ frequency, which is typical for the spectra of intrinsically disordered proteins.

\subsubsection{Crystal structure of the ctPrp2-ctSpp2 ${ }_{\mathrm{G}-\mathrm{patch}}$ complex}

Since all attempts to crystallize the yeast Prp2-Spp2 $2_{100-150}$ complex were fruitless, we used the ortholog proteins of Chaetomium thermophilum (ct), as this approach had previously been successful for the crystallization of other spliceosomal proteins like Prp2, Prp22, Prp43, Brr2 and Cwc27 (31, 33-38). The ctSpp2 consists of 313 residues, with the G-patch domain located between residues 211 and 254. For crystallization experiments ctSpp $2_{211-254}$ was generated and the stable complex of the truncated proteins ctPrp2 270-921 and ctSpp2 $211-254$ was prepared. Five different crystal forms (CF1-CF5) of this ctPrp2-ctSpp2 211-254 complex in four unique crystal packing arrangements were obtained, and all crystal structures were solved and refined.

In all crystal forms of the ctPrp2-ctSpp2 $211-254$ complex the N-terminal residues 212-222 of the G-patch domain form an amphipathic $\alpha$-helix that is bound to the WH domain (Figure 2). The $\alpha$-helix is terminated by a sharp kink followed by a region exhibiting an extended conformation reaching the $\beta$-hairpin of the RecA2 domain. The C-terminal part of the ctSpp2 G-patch adopts two alternative conformations in the five different crystal structures. In conformation 1 (CF1 and CF5) this part interacts with the upper region of the 
Chapter 3: Structural analysis of the interaction of Spp2 G-patch with Prp2

RecA2 domain and adopts a loop-like conformation. In both molecules of CF5 this section is preceded by a less than two turns long a-helical fragment not present in CF1, while in both crystal forms another short ahelical fragment is formed by the C-terminal residues of the G-patch. Formation of the $\alpha$-helical fragment present only in CF5 is most likely induced by crystal contacts with the G-patch of the symmetry-related molecule (Fig. S2). While in conformation 1 the polypeptide chain path is kinked at position Y238, in conformation 2 it forms a roughly straight extended conformation up to N240 followed by a turn redirecting it to bind to a similar region of the RecA2 domain as observed for the C-terminus of conformation 1 . The Gpatch of conformation 1 structures could be resolved up to D254 and in conformation 2 only residues up to R247 were interpretable in the electron density map.

The Spp2 G-patch binds Prp2 mainly via hydrophobic interactions (Figure 3B). The sequence alignment of Spp2 from C. thermophilum, S. cerevisiae and H. sapiens together with the S. cerevisiae G-patch domains of Prp43-binding G-patch proteins Ntr1, Gno1 and Pfa1 highlights the conservation of the hydrophobic residues of the N-terminal amphipathic a-helix and a hydrophobic stretch at the C-terminal end (Figure 3A). The hydrophobic side of the N-terminal a-helix face a highly conserved hydrophobic patch on the surface of the WH (Figure 3B). Both alternative C-terminal conformations bind the same conserved hydrophobic region on the RecA2 domain. Additionally to these hydrophobic interactions, the N-terminal a-helix is anchored via 棫stacking between F214(ctSpp2) and Y697(ctPrp2) and the two hydrogen bond pairs G215(ctSpp2)N694(ctPrp2) and R229(ctSpp2)-T686(ctPrp2) (Figure 3C). The conformation 1 is stabilized by polar interactions between L241(ctSpp2) and Y592(ctPrp2) as well as between K250(ctSpp2) and Y489(ctPrp2) constituting the C-terminal fragment of ctSpp2 $2_{211-254}$ (Figure 3D). Conformation 2 is stabilized by a hydrogen bond formed between G243(ctSpp2) and E486(ctPrp2) (Figure 3E).

Apart from a set of conserved glycines, the middle section of the G-patch connecting the N-terminal $\alpha$-helix with the C-terminal hydrophobic stretch shows a very low degree of conservation (Figure 3A). In all crystal forms except CF5 this linker region does not directly interact with Prp2 and exhibits significantly elevated $B$ factors implicating its larger flexibility (Fig. S3). B-factors of the linker in CF5 are not elevated as its conformation is stabilized by crystal contacts with the G-patch of a symmetry related complex (Fig. S2).

The functional impact of the conserved glycine residues being a hallmark of the G-patch has remained elusive so far. Analysis of the ctPrp2-ctSpp $2_{211-254}$ complex crystal structure revealed, that the observed combination of $\varphi$ and $\psi$ torsion angles of the Spp2 glycine residues 223, 226, 230 and 245, which are highly conserved throughout all G-patch proteins, are only favored for glycine residues according to the Ramachandran plot (Fig. S4).

In all five complex structures Prp2 contains a bound ADP molecule. A comparison with the previously reported structures of the Prp2-ADP complex reveals that the nucleotide conformation as well as the 
Chapter 3: Structural analysis of the interaction of Spp2 G-patch with Prp2

conformation of the Prp2 catalytic center are not affected by the binding of the G-patch (Fig. S5A) (35). This is also reflected in a virtually identical $K_{d}$ of about $170 \mathrm{nM}$ for the binding of ADP either in absence or in presence of the G-patch (Fig. S5B) (35). The binding of ATP in dependence of the G-patch could not be tested as non-hydrolyzable ATP analogs (AMPPNP, AMPPCP and ATPץS) did either not bind or were still hydrolyzed by Prp2. Although the interaction seems not to have a major influence on the global conformation of Prp2, a stabilization of the conformation of the RecA2 $\beta$-hairpin can be observed (Fig. S6).

\subsubsection{Cross-linking data confirm Spp2 binding site in solution}

In order to exclude the possibility that the binding mode of Spp2 observed in the crystal structure is solely induced by packing of the molecules in the crystal lattice, in-solution chemical cross-linking was performed on the complex of ctPrp2 with full-length ctSpp2, and cross-linked peptides were identified by mass spectrometry. Chemical cross-linking was performed with BS3, a lysine-directed homo-bifunctional crosslinking reagent with an $11.4 \AA$ spacer arm, as well as EDC, a hetero-bifunctional cross-linking reagent, that leads to the formation of a covalent bond between primary amines (Lys) and carboxyl containing amino acid residues (Asp, Glu).

Two out of the five lysine residues located within the G-patch and thus present in the crystal structure were cross-linked to ctPrp2, namely K236 and K250, which form cross-links to E646/D648 and K761 of ctPrp2 respectively. The first cross-link corresponds to a zero-length cross-link, while the second cross-link corresponds to lysine residues within the distance of the spacer arm of BS3. These results are in good agreement with the crystal structure of the ctPrp2-ctSpp2 $211-254$ complex (Figure 3F).

Since the in-solution cross-linking experiments were performed with full-length ctSpp2, five more crosslinks outside of the crystallized Spp2 fragment could be identified. These correspond to the lysine residues K17, K26, K72 and K296 in ctSpp2, cross-linked to the ctPrp2 residues K760, K761, K532/K535 and K761, respectively (Fig. S7A/B). Taken together, the cross-linking experiments with both reagents showed that the mode of binding of ctSpp $2_{211-254}$ to ctPrp2 in solution is maintained in the reported crystal structures of the complex, and that the interactions between both proteins extend beyond the crystallized ctSpp2 fragment. Cross-linking studies on the spliceosomal $\mathrm{B}^{\text {act }}$ complex from yeast also showed that regions other than the Gpatch of scSpp2 also interact with scPrp2 as well as other spliceosomal factors (27). 
Chapter 3: Structural analysis of the interaction of Spp2 G-patch with Prp2

\subsubsection{The G-patch of Spp2 populates transient helical conformations in solution}

To obtain residue-specific insight into the conformational space sampled by G-patch proteins in solution and prior to complex formation, a slightly larger ctSpp2 G-patch region (ctSpp2 $208-254$ ) was generated, as a higher yield of the purified recombinant protein could be obtained. Circular dichroism on ctSpp2 $208-254$ displayed a minimum around $203 \mathrm{~nm}$, typical of an unstructured protein that does not possess a stable secondary structure (Figure 4A), in agreement with the results for scSpp2 (Figure 1). In addition, dynamic light scattering showed that $\mathrm{ctSpp} 2_{208-254}$ is predominantly monomeric in solution (Figure 4A). To perform the sequence-specific assignment of its backbone resonances, the three-dimensional triple-resonance NMR experiments HNCA, HN(CO)CA, HNCACB, CBCA(CO)NH and HNCO were recorded using ${ }^{13} \mathrm{C} /{ }^{15} \mathrm{~N}-$ labeled ctSpp2 208-254. Analysis of the corresponding spectra enabled assignment of the backbone resonances of all non-proline residues of ctSpp2 $208-254$ (Figure 4B).

On the basis of the NMR chemical shifts the secondary structure of ctSpp $2_{208-254}$ was studied with singleresidue resolution. Particularly useful for this analysis are the $C^{\alpha}$ and $C^{\beta}$ chemical shifts (39). Figure $4 C$ shows the variation in $\Delta \delta \mathrm{C}^{\alpha}-\Delta \delta \mathrm{C}^{\beta}$ values along the primary sequence of ctSpp2 208 -254, that is the deviation between the experimental chemical shifts and those expected for a random coil peptide. Stretches of consecutive residues with positive $\Delta \delta C^{\alpha}-\Delta \delta C^{\beta}$ values are characteristic for the population of helical conformations, while regions with negative $\Delta \delta C^{\alpha}-\Delta \delta C^{\beta}$ values identify $\beta$-structure. For ctSpp $2_{208-254}$, the magnitude of $\Delta \delta C^{\alpha}-\Delta \delta C^{\beta}$ values was below 1.0 (Figure $4 \mathrm{C}$ ), in agreement with the absence of rigid secondary structure (Figure $4 \mathrm{~A}$ ), which would result in $\Delta \delta \mathrm{C}^{\alpha}-\Delta \delta \mathrm{C}^{\beta}$ values $>3.0$. At the same time, a pronounced tendency of residues F214-S221 to populate helical conformations was detected (Figure 4C). Quantitative analysis of the $\Delta \delta C^{\alpha}-\Delta \delta C^{\beta}$ values indicated that about $20 \%$ of the conformations sampled by F214-S221 are helical (40).

Next, the dynamic properties of ctSpp2 $208-254$ were investigated. Subjecting the assigned chemical shifts to the software TALOS+ (41) provided estimates for the general order parameter $\left(S^{2}\right)$. $S^{2}$ values describe the extent of motions on a ps-ns time scale, providing important information on the level of spatial restriction within the molecular reference frame. Low order parameters throughout the backbone of ctSpp $2_{208-254}$ were found (Figure 4C), indicating a low motional restriction typical for IDPs. The least dynamic residues were F214S221, which transiently populate helical structure. The decreased mobility of this region was further supported by ${ }^{15} \mathrm{~N}$ spin relaxation measurements. The $\mathrm{N}$-terminus and the C-terminal residues starting at residue L244 had negative steady-state $\left\{{ }^{1} \mathrm{H},{ }^{15} \mathrm{~N}\right\}$ nuclear Overhauser effect (NOE) values (Figure 4D), indicating that they are the most dynamic parts of ctSpp $2_{208-254}$. In between, steady-state $\left\{{ }^{1} \mathrm{H},{ }^{15} \mathrm{~N}\right\}$ NOE values were approximately 0.25 . Notably, residues F214-S221 did not have higher steady-state $\left\{{ }^{1} \mathrm{H},{ }^{15} \mathrm{~N}\right\}$ NOE values than the following residues up to A239 (Figure 4D), suggesting that the elevated general order 
Chapter 3: Structural analysis of the interaction of Spp2 G-patch with Prp2

parameters $S^{2}$ of F214-S221 are caused by slower motions, which affect the chemical shifts but not the steady-state $\left\{{ }^{1} \mathrm{H},{ }^{15} \mathrm{~N}\right\}$ NOE. Such slower motions are consistent with transient helix formation. Taken together, NMR spectroscopy demonstrates that the conserved N-terminal part of the G-patch of ctSpp2 populates transient helical conformations in solution and a part of the conserved hydrophobic C-terminal stretch shows increased dynamics in agreement with the alternative conformations of this region found in the crystal structures (Figure 4E).

\subsection{Discussion}

G-patch proteins play a vital role in the functioning of the spliceosomal DEAH-box ATPases Prp2 and Prp43 and their interplay has thus been subject of numerous studies. While in both cases the interaction of the Gpatch protein with the corresponding ATPase is essential to fulfill their roles in the spliceosomal context, they modulate the activity of its ATPase differently $(18,19,23,42)$. Spp2 enhances the ATPase activity of Prp2 only in the presence of RNA and is not able to induce any helicase activity $(24,25)$. In contrast, Ntr1 stimulates the Prp43 ATPase activity in presence and in absence of RNA, and greatly enhances the helicase activity $(22,23,37)$. Although the functional impact of the G-patch proteins on the spliceosomal DEAH-box ATPases and ultimately its role during splicing has been extensively studied, not much about their interaction mode is known. It has been demonstrated that a stretch of sixty amino acids containing the conserved Gpatch domain of Ntr1 is sufficient for Prp43 interaction and stimulation (22, 43). With this information we designed a minimal Spp2 construct to gain molecular insights into the interaction of the G-patch with the DEAH-box ATPase Prp2 by means of X-ray crystallography and to study its properties in solution using CD and NMR spectroscopy.

Our data show that both, N-terminally truncated scSpp2 ${ }_{10-185}$ and the G-patch domains of scSpp2 and ctSpp2, are intrinsically disordered in solution (Figure 1, Figure 4). However, in the ctPrp2-ctSpp2 $211-254$ complex, the $\mathrm{N}$-terminal region of the G-patch adopts a helical conformation, which could be confirmed to be transiently present in only $20 \%$ of the molecules in solution (Figure 2, Figure 4). This amphipathic a-helix binds primarily via hydrophobic interactions to the ctPrp2 winged-helix domain in all crystal structures (Figure 3). The C-terminal end of the crystallized G-patch exhibits two alternative conformations in different crystal structures, both of which mainly bind to the RecA2 domain also via hydrophobic interactions (Figure 2, Figure 3). This C-terminal hydrophobic stretch and the N-terminal $\alpha$-helix are connected by a linker region that does not directly interact with ctPrp2. Elevated B-factor values in CF1-4 suggest a high flexibility of this region (Fig. S3). 
Chapter 3: Structural analysis of the interaction of Spp2 G-patch with Prp2

The G-patch regions of Spp2 and Ntr1 share the property of being intrinsically disordered in solution (22). Interestingly, CD spectra of the Ntr1 G-patch showed no secondary structures, but formation of a-helices could be induced upon addition of trifluorethanol (TFE). These helices could likely correspond to the Nterminal a-helix observed for ctSpp2 $211-254$ upon interaction with ctPrp2. In fact, this part of the G-patch is highly conserved in G-patch proteins interacting with either Prp2 or Prp43 and the presence of hydrophobic residues occurring in a 3-4 pattern $(\mathrm{HxxH}, \mathrm{HxxxH})$ is strongly arguing in favor of the formation of an amphipathic a-helix as well in all G-patch proteins interacting with Prp43 (Figure 3A). The C-terminal end shows also a high degree of conservation of hydrophobic residues and glycines. The flexible linker region of Spp2 does not show any sequence conservation, except for a set of glycine residues. The occurrence of these glycines in all G-patch domains might play a role in maintaining the flexibility of this linker. Additionally, the hydrophobic patches on ctPrp2 involved in the interaction with the N- and C-terminal parts of ctSpp2 211 254 are also conserved among Prp2 and Prp43 (Fig. S8). The level of conservation of the interacting regions on both, Prp2/Prp43 and the different G-patch domains, suggests that the interactions of G-patchs with Prp43 might be comparable to the one seen in the ctPrp2-ctSpp $2_{211-254}$ complex. In fact, a previously published study on the scPrp43-scNtr1 interaction proposes a binding site which is similar to the one seen in the ctPrp2Spp2 $211-254$ complex structure (22). By superposition-based modeling of ctSpp2 $211-254$ onto the ADP-bound scPrp43 structure, several of the identified cross-links between scPrp43 and scNtr1 could be confirmed to be in good agreement with respect to the observed distances in the model, thereby suggesting a similar binding mode as seen for ctSpp2 $211-254$ and ctPrp2 (Fig. S9).

Both, Spp2 and Ntr1, have been proposed to bind to the C-terminal domain of their respective ATPase and the OB-fold domain was accredited a special role in this interaction $(19,22,44)$. While we can confirm that the N-terminal a-helix of the G-patch indeed binds to the winged-helix domain which is part of the Cterminal domain, our complex structure does not show any direct interactions between ctSpp2 $211-254$ and the OB-fold domain (Figure 2, Figure 3). However, the C-terminal stretch of the ctSpp2 G-patch binds to a hydrophobic patch on the RecA2 domain located at the interface between the OB-fold and the RecA2 domain. Conformational perturbations due to mutations introduced into the OB-fold domain (19) or even the complete truncation of this domain (44) could indirectly lead to the observed impacts in binding and function of the G-patch protein. In particular, the $\beta$-hairpin of the RecA2 domain, which is involved in numerous contacts with the OB-fold domain, may be structurally influenced by artificial variations of the OB-fold domain. This could lead to an indirect effect on the binding of the G-patch, since its linker region extends close to this structural feature.

Cryo-EM structures of the $\mathrm{B}^{\text {act }}$ complex unveiled the location of Prp2 within the spliceosome, but due to the high flexibility of Spp2 and the limited resolution of cryo-EM 3D reconstructions at the periphery of such dynamic complexes, no structural information of the G-patch protein could be gained by this technique so far 
Chapter 3: Structural analysis of the interaction of Spp2 G-patch with Prp2

(27, 28, 45, 46). Using the presented ctPrp2-ctSpp2 $2_{211-254}$ complex structure it is now possible to situate the Spp2 G-patch within the $\mathrm{B}^{\text {act }}$ complex and in concert with available cross-link data estimate the interaction network of flanking regions of the G-patch (Fig. S10) (27). The association of Spp2 with the spliceosome can be divided into three different interaction regions. The N-terminal part contacts spliceosomal factors Brr2 and Rse1 as well as Prp2 (Figure 5A/B). The interaction between Spp2 and Prp2 at this site seems to be highly dynamic as multiple cross-links were found on the ATPase. The Spp2 G-patch likely plays the role of the major anchor point of Spp2 on Prp2 and extends from the winged-helix domain up to the RecA2 domain. Cross-links between the C-terminal end of the G-patch and the OB-fold domain located in close proximity are in good agreement with the ctPrp2-ctSpp2 $211-254$ complex structure (Figure 5B/C). Additionally, this part of the G-patch contacts nearby regions of Bud13 and Pml1, which are not visible in the cryo-EM 3D reconstruction. C-terminally from G-patch, Spp2 contacts Prp2 at a more distant location of the RecA2 domain. The numerous interactions of Spp2 with Prp2 and the spliceosomal factors Brr2, Rse1, Bud13 and Pml1 might play a role in the proper positioning of the ATPase after recruitment to the spliceosome. In summary, the cross-link data published by Rauhut et al. (2016) are supported by our ctPrp2-ctSpp2 $211-254$ complex structure as the relative orientation of the G-patch is in good agreement with these findings. Additionally, our cross-link data confirm that also parts outside the ctSpp2 G-patch can contact ctPrp2, as seen for S. cerevisiae Spp2 and Prp2. (Fig. S7).

The intrinsic flexibility of proteins is known to be connected to many advantages in binding to interaction partners, like increased adaptability and specificity, and in the case of G-patch proteins this flexibility seems to be crucial for coping with the conformational dynamics of Prp2 and Prp43 (1-3). It has been shown that during the RNA translocation of DEAH-box ATPases, the RecA2 domain is the most mobile domain, rotating $19^{\circ}$ upon ATP hydrolysis, moving away from the RecA1 domain by $6.5 \AA$ after ADP release and returning to a closed conformation upon ATP binding (Figure 5D) (31). Since the C-terminal hydrophobic stretch of ctSpp2 $211-254$ is located on the RecA2 domain of Prp2, it has to be able to adapt to the movements of the RecA2 domain during translocation in order to remain constantly bound. On the one hand, this is achieved by the presence of a flexible linker connecting the anchoring $\alpha$-helix with the C-terminal interacting fragment of the G-patch and, on the other hand, by a highly flexible interaction mode of the Cterminal ctSpp2 $211-254$ region with the ctPrp2 RecA2 domain (Figure 3B; Fig. S3). The binding at this site relies mainly on hydrophobic interactions, which has the advantage that only few polar interactions have to be broken upon conformational changes (Figures 3D/E). This allows this part of the G-patch to adopt different alternative conformations as seen in the crystal structures (Figure 2). Additionally, NMR data indicate that even in solution the C-terminal part of the ctSpp2 G-patch exhibits an increased flexibility, consistent with the alternative conformations observed in the crystal structures (Figure 4D). Although we were able to observe two different interaction modes of this region in the ADP-bound state of Prp2, other 
Chapter 3: Structural analysis of the interaction of Spp2 G-patch with Prp2

conformations might occur in different nucleotide-bound states of Prp2. Conserved glycines at the beginning of the linker and in the C-terminal hydrophobic stretch likely enhance the flexible properties of these two regions and ultimately ensure that the C-terminal end of the G-patch is able to stay in contact with the hydrophobic patch on the RecA2 domain at every nucleotide-specific conformational state of the DEAH-box ATPase (Figure 3A; Figure 5D). In contrast, the N-terminal amphipathic a-helix of the G-patch shows no variability in binding to the winged-helix domain in all crystal structures (Figure 2). In addition, NMR studies indicate that the flexibility of these residues is lower when compared to other regions of the G-patch (Figure 4C). This favors the binding of the ctSpp2 G-patch to ctPrp2, because the entropic penalty that the G-patch has to pay when it assumes a rigid conformation in the bound state is decreased. Thus, crystallographic and NMR data suggest that the N-terminal part of the G-patch acts as the main anchoring point to Prp2. Although this part of the G-patch transiently samples helical conformations with an average of $20 \%$ (ensemble and time average), it does not provide enough evidence for a binding based on conformational selection (Figure 4E). Assuming this case, Prp2 might be able to select and further stabilize already transiently populated helical conformations of the N-terminal part and the C-terminal parts could subsequently bind to Prp2 as well. Instead, a folding upon binding mechanism cannot be excluded as the majority of the Spp2 G-patch is not found in the pre-folded state.

Due to the stimulating effect of G-patch proteins on the functions of Prp2 and Prp43 it has also been speculated that the G-patch might directly interact with either the ATP- or RNA-binding site (22, 24). Our ctPrp2-ctSpp2 $211-254$ complex structures can confirm that the G-patch does not bind anywhere close to the adenosine nucleotide-binding site and thus is not able to directly alter the catalytic center (Figure 2). In contrast, the C-terminal end of the Spp2 G-patch is located in close proximity to the entrance of the RNAbinding tunnel as seen in the RNA complex structures of Prp43 and Prp22 (31, 37). However, assuming the same binding of the C-terminal end to the RecA2 domain in the ATP and Apo states, this part of the G-patch is still about 12-13 $\AA$ distant to the closest RNA backbone phosphate (Figure S11). This distance makes an involvement of the G-patch in interactions with the RNA unlikely. Instead of directly interacting and altering binding sites for ATP/ADP and RNA, the G-patch might have an effect on the global dynamics of the DEAH-box ATPase and thus affect functional properties like ligand binding and catalytic efficiency in a more indirect manner. For example, the N-terminal amphipathic $\alpha$-helix binds to the winged-helix domain, which serves as hinge region for the opening of the RNA-binding tunnel (Figure 2) (37). This interaction might have an impact on the binding of the RNA, which in turn could lead to the observed RNA-dependent stimulation of the ATPase activity of Prp2 and Prp43 by its respective G-patch protein (22, 24). On the other side, the C-terminal part of the G-patch interacts with the highly mobile RecA2 domain and might affect the mobility of this domain (Figure 5D) (31). Additionally, although the linker region of the G-patch does not interact with the $\beta$-hairpin of the RecA2 domain, it folds over this structural feature like a safety belt in all 
Chapter 3: Structural analysis of the interaction of Spp2 G-patch with Prp2

ctPrp2-ctSpp2 211-254 complex structures and stabilizes its conformation, which has been found to be rather flexible in the absence of Spp2 (Fig. S6) (35). Since the mobility of the RecA2 is tightly linked to ATP hydrolysis, influences by the G-patch on the dynamics of this domain might have a regulatory effect on binding constants of ATP/ADP as well as on the catalysis rate of ATP. In order to get more in depth insights into the exact modus operandi of the G-patch proteins extensive studies using other techniques are needed. Single-molecule experiments as well as stopped-flow kinetics with Prp2 and Prp43 in presence and absence of their respective G-patch proteins could provide more detailed information about the functional implications and complement our structural data.

\subsection{Materials and methods}

\subsubsection{Protein expression and purification}

For expression of Saccharomyces cerevisiae Spp2 (scSpp2), cDNA encoding truncated proteins comprising either residues 10 - 185 or the G-patch containing region 100 - 150 were cloned into pET-21a vector. The recombinant proteins with C-terminal hexa-histidine tag were expressed in Escherichia coli Rosetta II (DE3) at $16{ }^{\circ} \mathrm{C}$. Cells were disrupted in $50 \mathrm{mM}$ HEPES/NaOH (pH 7.5), $500 \mathrm{mM} \mathrm{NaCl}, 5 \%$ (v/v) glycerol, $2 \mathrm{mM}$ $\beta$-mercaptoethanol and lysate was clarified by centrifugation for 30 minutes at 30,000 xg. The proteins were purified at $20^{\circ} \mathrm{C}$ on a Ni-NTA-Sepharose column (in $50 \mathrm{mM}$ HEPES/NaOH (pH 7.5), $500 \mathrm{mM} \mathrm{NaCl}, 5 \%$ (v/v) glycerol, $30 \mathrm{mM}$ imidazole, $2 \mathrm{mM} \beta$-mercaptoethanol) including a wash step with binding buffer supplemented with $2 \mathrm{M} \mathrm{LiCl}$ to remove bound nucleic acids. The buffer was exchanged against $10 \mathrm{mM}$ HEPES/NaOH (pH 7.5), $50 \mathrm{mM} \mathrm{NaCl}, 5 \%$ (v/v) glycerol, $2 \mathrm{mM} \beta$-mercaptoethanol using a HiTrap desalting column (GE Healthcare). Further purification was obtained by anion-exchange chromatography using a Source30Q column. Finally, the protein was purified by a Superdex-75 gelfiltration column (GE Healthcare) in a buffer containing $10 \mathrm{mM}$ HEPES/NaOH (pH 7.5), $100 \mathrm{mM} \mathrm{NaCl}, 5 \%$ (v/v) glycerol, $2 \mathrm{mM} \beta-$ mercaptoethanol. The protein was concentrated to $10 \mathrm{mg} / \mathrm{ml}$ using an Amicon Ultra centrifugal concentrator (Merck) in 10 mM HEPES/NaOH (pH 7.5), $200 \mathrm{mM} \mathrm{NaCl,} \mathrm{5 \%} \mathrm{(v/v)} \mathrm{glycerol} \mathrm{and} 2 \mathrm{mM} \beta$-mercaptoethanol. For NMR-experiments, scSpp2 ${ }_{10-185}$ was expressed in M9 minimal media supplemented with ${ }^{15} \mathrm{NH}_{4} \mathrm{Cl}$. Purification of the N15-labeled scSpp2 was performed as described above.

Spp2 from Chaetomium thermophilum (ctSpp2) was identified by NCBI BLAST search tool (GenBank: EGS17798.1) and the gene encoding for the full-length protein was amplified by PCR from a total DNA preparation. The PCR product was cloned into the IBA Stargate pASG-IBA-3 vector according to the manufacturer's protocol. The Strep-tagged recombinant protein was expressed in Escherichia coli Rosetta II (DE3) at $16^{\circ} \mathrm{C}$. Cells were disrupted using a fluidizer (Microfluidics) in $50 \mathrm{mM}$ HEPES/ $\mathrm{NaOH}$ (pH 7.5), 500 
Chapter 3: Structural analysis of the interaction of Spp2 G-patch with Prp2

$\mathrm{mM} \mathrm{NaCl}, 5 \%(\mathrm{v} / \mathrm{v})$ glycerol, $1 \mathrm{mM}$ DTT and lysate was clarified by centrifugation for 30 minutes at 30,000 xg. The protein was purified at $20^{\circ} \mathrm{C}$ on a Streptactin-Sepharose column (in $50 \mathrm{mM}$ HEPES/NaOH (pH 7.5), $500 \mathrm{mM} \mathrm{NaCl}, 5 \%$ (v/v) glycerol, $1 \mathrm{mM} \mathrm{DTT}$ ). Further purification was obtained by a Superdex-75 gelfiltration column. The protein was concentrated to $20 \mathrm{mg} / \mathrm{ml}$ in $10 \mathrm{mM}$ HEPES/NaOH (pH 7.5), $150 \mathrm{mM}$ $\mathrm{NaCl}$, and $1 \mathrm{mM}$ DTT.

For expression of truncated ctSpp2 comprising G-patch residues 211 - 254, the encoding gene was amplified by PCR from a total DNA preparation (Altschul et al, 1990). The PCR product was cloned into the IBA Stargate pASG-IBA-25 vector according to the manufacturer's protocol. Expression and purification by Glutathion-Speharose was performed as described for scSpp2. Further purification was achieved by a Superdex-75 gelfiltration column. The protein was concentrated to $20 \mathrm{mg} / \mathrm{ml}$ in $10 \mathrm{mM}$ HEPES/NaOH (pH 7.5), $150 \mathrm{mM} \mathrm{NaCl}$, and $1 \mathrm{mM}$ DTT.

For NMR studies a construct of the ctSpp2 G-patch comprising residues 208 - 254 was cloned into the plasmid pASG-IBA 25, which was transformed into BL21 (DE3) cells. ctSpp2 $208-254$ was expressed in M9 minimal medium for the production of ${ }^{15} \mathrm{~N}$ - and ${ }^{15} \mathrm{~N} /{ }^{13} \mathrm{C}$-labelled ctSpp $2_{208-254}$. Purification of this construct was performed as described for ctSpp2 $211-254$, with the exception of the gelfiltration buffer containing $10 \mathrm{mM}$ HEPES/NaOH pH 7.5, $200 \mathrm{mM} \mathrm{NaCl,} \mathrm{5 \%} \mathrm{Glycerol,} 1 \mathrm{mM}$ DTT and 0.02\% $\mathrm{NaN}_{3}$. Prior to NMR studies the buffer was exchanged to $10 \mathrm{mM}$ HEPES/NaOH pH 6.5, $200 \mathrm{mM} \mathrm{NaCl}$, 0.5\% Glycerol, $1 \mathrm{mM}$ DTT, $5 \mathrm{mM}$ EDTA and $0.02 \% \mathrm{NaN}_{3}$.

ctPrp2 was expressed and purified as described in (35).

\subsubsection{Far-UV circular dichroism spectroscopy}

scSpp $2_{10-185}$ and scSpp2 $2_{100-150}$ samples were dialyzed against buffer containing $100 \mathrm{mM}$ sodium phosphate $\mathrm{pH}$ 7.5 and diluted to $0.1 \mathrm{mg} / \mathrm{ml}$. ctSpp2 $208-254$ was dialyzed in pure water and diluted to a final concentration of $0.2 \mathrm{mg} / \mathrm{ml} .200 \mu \mathrm{L}$ of the sample were pipetted into a cuvette with a path length of $1 \mathrm{~mm}$ and transferred into a Chirascan CD spectrometer (AppliedPhotophysics). The spectra were recorded in the far UV range from $190-260 \mathrm{~nm}$ at $20^{\circ} \mathrm{C}$ with a time-per-point value of $1 \mathrm{~s}$ and a path length of $1 \mathrm{~nm}$. Every measurement was performed three times. For the final absorption curve, the values of the negative control, which contained exclusively the sample buffer or pure water, were subtracted from the values of the protein sample. 
Chapter 3: Structural analysis of the interaction of Spp2 G-patch with Prp2

\subsubsection{NMR spectroscopy}

NMR experiments on scSpp2 $2_{10-185}$ and scSpp2 $2_{100-150}$ were measured at $288 \mathrm{~K}$ on Bruker $600 \mathrm{MHz}$ spectrometers equipped with cryoprobes. The $2 \mathrm{D}{ }^{1} \mathrm{H}-{ }^{15} \mathrm{~N}$ HSQC was recorded at $288 \mathrm{~K}$ with $1024(\mathrm{H}) * 300(\mathrm{~N})$ complex points. Four transients were accumulated for each FID. Spectra were processed using NMRPipe (48) and analyzed using Sparky (49).

NMR experiments on ctSpp2 $208-254$ were performed on a $700 \mathrm{MHz}$ spectrometer (Bruker) equipped with a cryogenic probe. Three-dimensional triple resonance experiments HNCA, HN(CO)CA, HNCACB, CBCA(CO)NH and HNCO (50) were recorded at $298 \mathrm{~K}$ on ${ }^{15} \mathrm{~N}$, ${ }^{13} \mathrm{C}$-labelled ctSpp 2 208-254. Steady-state heteronuclear $\left\{{ }^{1} \mathrm{H},{ }^{15} \mathrm{~N}\right\}$-NOE values were acquired at $298 \mathrm{~K}$ with and without ${ }^{1} \mathrm{H}$ presaturation, with a recycle delay of 4 s. Spectra were processed with Topspin (Bruker) and NMRPipe (48) and analyzed with ccpnmr Analysis 2.2.1 (51). All NMR experiments were acquired at a protein concentration of $0.52 \mathrm{mM}$.

The structural ensemble of ctSpp $2_{208-254}$ was calculated using flexible-meccano assuming an a-helical propensity of $20 \%$ for residues F214-S221, which was determined from the experimental ${ }^{13} \mathrm{C}^{\alpha}$ and ${ }^{13} \mathrm{C}^{\beta}$ chemical shift as described by Marsh et al. (40).

\subsubsection{Dynamic light scattering}

Dynamic light scattering was performed on a sample of ctSpp $2_{208-254}$ (concentration of $0.2 \mathrm{mM}$ ) at $25^{\circ} \mathrm{C}$ both in water and in $10 \mathrm{mM}$ HEPES/NaOH pH 6.5, $200 \mathrm{mM} \mathrm{NaCl}$, 0.5\% Glycerol, $1 \mathrm{mM}$ DTT, 5 mM EDTA and $0.02 \% \mathrm{NaN}_{3}$. Measurements were acquired in triplicates on a Dynamic proTitan DLS instrument equipped with a temperature control system (Wyatt technology corporation) with a power laser strength of $60 \%$. Samples were allowed to equilibrate for one minute before starting the acquisition.

\subsubsection{Crystallization}

Crystals of crystal forms $1-4$ were grown at $20^{\circ} \mathrm{C}$ by sitting drop vapor diffusion after mixing $1 \mu$ l of reservoir solution with $1 \mu \mathrm{l}$ of $2.5 \mathrm{mg} / \mathrm{ml}$ ctPrp2 supplemented with a 2 -fold excess of ctSpp $2_{211-254}, 10$-fold molar excess of ADP, a 20-fold molar excess of $\mathrm{BeSO}_{4}$, a 60 -fold molar excess $\mathrm{NaF}$ and a 2.5-fold molar excess of $U_{12}$-RNA (AXOlabs, Germany). Crystals of CF1 were obtained in $100 \mathrm{mM}$ MES pH 6.5, $350 \mathrm{mM}$ calcium acetate and 19\% (w/v) PEG4000; crystals of CF2 in 100 mM Bis-Tris pH 5.5, 400 mM ammonium sulfate and 23\% (w/v) PEG3350; crystals of CF3 in $100 \mathrm{mM}$ sodium cacodylate $\mathrm{pH}$ 6, $33 \mathrm{mM} \mathrm{KCl,} \mathrm{12 \%}$ (v/v) isopropyl alcohol and $25 \mathrm{mM} \mathrm{MgCl}_{2}$ and crystals of CF4 in $100 \mathrm{mM} \mathrm{HEPES/NaOH} \mathrm{pH} \mathrm{7,} \mathrm{8 \%} \mathrm{(w/v)}$ 
Chapter 3: Structural analysis of the interaction of Spp2 G-patch with Prp2

polyvinyl alcohol and 7.5\% (v/v) isopropyl alcohol. Crystals of CF5 were grown at $20^{\circ} \mathrm{C}$ by sitting drop vapor diffusion after mixing $1 \mu \mathrm{l}$ of reservoir solution with $2 \mu \mathrm{l}$ of $3.5 \mathrm{mg} / \mathrm{ml} \mathrm{ctPrp} 2$ supplemented with 3-fold molar excess of ctSpp $2_{211-254}$ and a 10 -fold molar excess of ADP. CF5 crstals were obtained in $100 \mathrm{mM}$ Bicine/Trizma pH 7.5, 11\% (w/v) PEG8000, 20\% (w/v) ethylene glycol, $20 \mathrm{mM}$ of a monosaccharide mixture (D-glucose, D-mannose, D-galactose, L-fucose, D-xylose and N-acetyl-D-glucosamine).

\subsubsection{Data collection and processing}

Crystals of CF1 and CF2 were cryoprotected in the respective reservoir solution complemented with $10 \%$ (w/v) glycerol, CF3 with 40\% (w/v) glycerol, CF4 with 25\% glycerol and CF5 was not additionally cryoprotected. X-ray diffraction data of CF1, CF2 and CF5 were collected at $100 \mathrm{~K}$ on beamline P13 and CF3 as well as CF4 were collected at beamline P14, PETRA III, DESY (Hamburg, Germany). Processing of the diffraction data was performed with the XDS package (52). The highest resolution limit was estimated using a minimum $\mathrm{I} / \sigma(\mathrm{I})$ of 1.5 and a minimum $\mathrm{CC}_{1 / 2}$ of $60 \%$ as cutting criteria. X-ray diffraction data statistics are summarized in Table 1.

\subsubsection{Structure solution, refinement and analysis}

All crystal forms of the ctPrp2-ctSpp2 complex were solved by molecular replacement using Phaser with the structure of ctPrp2 (PDBid: 6fa5) as a search model $(35,53)$. After an initial refinement with PHENIX, the ctSpp2 G-patch could be built manually with Coot using the $\mathrm{mF}_{\mathrm{o}}-\mathrm{DF}_{\mathrm{c}}$ map $(54,55)$. All consecutive refinement cycles were performed with PHENIX including TLS, weight optimization and bulk-solvent optimization. The quality of the final models was assessed MolProbity and with the validation tools in PHENIX (56). The figures were prepared with PyMOL (v.1.8; Schrödinger).

\subsubsection{Protein-protein cross-linking}

The optimal cross-linker to protein ratio was determined by using $2.5 \mu \mathrm{g}$ aliquots of the ctPrp2/ctSpp2 complex and a series of cross-linker molar excesses of 5, 10, 25, 50, 100 and 200 as well as a control. Samples were allowed to react with freshly prepared BS3 for 30 minutes at room temperature. The chosen cross-linker to protein ratio was 75 , which led to a high yield, yet to a homogeneous sharp band as visualized on SDS-PAGE. The cross-linked samples were analyzed by SDS page on a 4-12\% Bis-Tris gel (Invitrogen) with MES as running buffer and stained with Coomassie blue. 
Chapter 3: Structural analysis of the interaction of Spp2 G-patch with Prp2

For MS analysis, the ctPrp2/ctSpp2 mixture was cross-linked with freshly prepared BS3 and EDC (Pierce, Thermo Scientific) in a 75:1 cross-linker to protein ratio, allowed to react and separated as described above. Cross-linked bands were excised from the gel and combined in a single reaction cup according to crosslinking reagent. In gel digestion and extraction of peptides was achieved as described elsewhere (57). The solution of extracted peptides was concentrated on a vacuum evaporator to $5 \mu \mathrm{L}$ and diluted to a final volume of $10 \mu \mathrm{l}$ to reach a sample solvent composition of 5\% (v/v) CAN, 1\% (v/v) FA. Samples were submitted for immediate analysis to the mass spectrometer. Mass spectrometric analysis and cross-link identification were performed as described elsewhere (22).

\subsubsection{Isothermal titration calorimetry (ITC)}

The binding of ADP to ctPrp2 in the presence of ctSpp2 $211-254$ was assessed via ITC with a MicroCal VP-ITC (Malvern) using a ctPrp2 and ctSpp2 $211-254$ concentration of $10 \mu \mathrm{M}$ and $20 \mu \mathrm{M}$, respectively, in the cell and a concentration of $100 \mu \mathrm{M}$ ADP in the syringe. A reaction buffer consisting of $20 \mathrm{mM}$ HEPES pH 7.5, $200 \mathrm{mM} \mathrm{NaCl}, 5 \%$ glycerol and $2 \mathrm{mM} \mathrm{MgCl}_{2}$ was used. Each measurement consisted of an initial $6 \mu \mathrm{l}$ injection and injections with a volume of $14 \mu \mathrm{l}$ injected with a speed of $1 \mu \mathrm{s} \mathrm{s}^{-1}$. The interval between each injection was set to $250 \mathrm{~s}$ and the binding was monitored at $20^{\circ} \mathrm{C}$. NITPIC was utilized for data integration, the integrated data were processed and analyzed with SEDPHAT and for the final representation of the data GUSSI was used (58-60).

\section{Acknowledgments}

We acknowledge DESY (Hamburg, Germany), a member of the Helmholtz Association HGF, for the provision of experimental facilities. Parts of the research were carried out at PETRA III and we are thankful for the assistance using EMBL beamlines P13 and P14. We thank Dr. Stefan Becker for help with the production of ctSpp $2_{208-254}$. This work was supported by grants of the Deutsche Forschungsgemeinschaft (DFG) to R.F., H.U., M.Z. (SFB860 TP A02, A10, B02, Z01). 
Chapter 3: Structural analysis of the interaction of Spp2 G-patch with Prp2

\section{References}

1. Dyson HJ, Wright PE (2005) Intrinsically unstructured proteins and their functions. Nat Rev Mol Cell Biol 6(3):197-208.

2. Stein A, Pache RA, Bernadó P, Pons M, Aloy P (2009) Dynamic interactions of proteins in complex networks: a more structured view. FEBS J 276(19):5390-5405.

3. Tompa P (2009) Structure and Function of Intrinsically Disordered Proteins. CRC Press. Available at: https://www.crcpress.com/Structure-and-Function-of-Intrinsically-Disordered-Proteins/TompaFersht/p/book/9781420078923 [Accessed March 17, 2019].

4. Korneta I, Bujnicki JM (2012) Intrinsic disorder in the human spliceosomal proteome. PLoS Comput Biol 8(8):e1002641.

5. Hoskins AA, Moore MJ (2012) The spliceosome: a flexible, reversible macromolecular machine. Trends Biochem Sci 37(5):179-188.

6. Matera AG, Wang Z (2014) A day in the life of the spliceosome. Nat Rev Mol Cell Biol 15(2):108121.

7. Will CL, Lührmann R (2011) Spliceosome structure and function. Cold Spring Harb Perspect Biol 3(7). doi:10.1101/cshperspect.a003707.

8. Wahl MC, Will CL, Lührmann R (2009) The spliceosome: design principles of a dynamic RNP machine. Cell 136(4):701-718.

9. Fabrizio P, et al. (2009) The evolutionarily conserved core design of the catalytic activation step of the yeast spliceosome. Mol Cell 36(4):593-608.

10. Cordin O, Hahn D, Beggs JD (2012) Structure, function and regulation of spliceosomal RNA helicases. Curr Opin Cell Biol 24(3):431-438.

11. Ding SC, Pyle AM (2012) Molecular mechanics of RNA translocases. Meth Enzymol 511:131-147.

12. Ozgur S, et al. (2015) The conformational plasticity of eukaryotic RNA-dependent ATPases. FEBS $J$ 282(5):850-863.

13. Fairman-Williams ME, Guenther U-P, Jankowsky E (2010) SF1 and SF2 helicases: family matters. Curr Opin Struct Biol 20(3):313-324.

14. Cordin O, Beggs JD (2013) RNA helicases in splicing. RNA Biol 10(1):83-95.

15. Kudlinzki D, Schmitt A, Christian H, Ficner R (2012) Structural analysis of the C-terminal domain of the spliceosomal helicase Prp22. Biol Chem 393(10):1131-1140.

16. Kim SH, Lin RJ (1996) Spliceosome activation by PRP2 ATPase prior to the first transesterification reaction of pre-mRNA splicing. Mol Cell Biol 16(12):6810-6819.

17. King DS, Beggs JD (1990) Interactions of PRP2 protein with pre-mRNA splicing complexes in Saccharomyces cerevisiae. Nucleic Acids Res 18(22):6559-6564. 
Chapter 3: Structural analysis of the interaction of Spp2 G-patch with Prp2

18. Roy J, Kim K, Maddock JR, Anthony JG, Woolford JL (1995) The final stages of spliceosome maturation require Spp2p that can interact with the DEAH box protein Prp2p and promote step 1 of splicing. RNA 1(4):375-390.

19. Silverman EJ, et al. (2004) Interaction between a G-patch protein and a spliceosomal DEXD/H-box ATPase that is critical for splicing. Mol Cell Biol 24(23):10101-10110.

20. Aravind L, Koonin EV (1999) G-patch: a new conserved domain in eukaryotic RNA-processing proteins and type D retroviral polyproteins. Trends Biochem Sci 24(9):342-344.

21. Robert-Paganin J, Réty S, Leulliot N (2015) Regulation of DEAH/RHA helicases by G-patch proteins. Biomed Res Int 2015:931857.

22. Christian H, Hofele RV, Urlaub H, Ficner R (2014) Insights into the activation of the helicase Prp43 by biochemical studies and structural mass spectrometry. Nucleic Acids Res 42(2):1162-1179.

23. Tanaka N, Aronova A, Schwer B (2007) Ntr1 activates the Prp43 helicase to trigger release of lariatintron from the spliceosome. Genes Dev 21(18):2312-2325.

24. Warkocki Z, et al. (2015) The G-patch protein Spp2 couples the spliceosome-stimulated ATPase activity of the DEAH-box protein Prp2 to catalytic activation of the spliceosome. Genes Dev 29(1):94-107.

25. Bao P, Höbartner C, Hartmuth K, Lührmann R (2017) Yeast Prp2 liberates the 5' splice site and the branch site adenosine for catalysis of pre-mRNA splicing. RNA 23(12):1770-1779.

26. Kim SH, Smith J, Claude A, Lin RJ (1992) The purified yeast pre-mRNA splicing factor PRP2 is an RNA-dependent NTPase. EMBO J 11(6):2319-2326.

27. Rauhut R, et al. (2016) Molecular architecture of the Saccharomyces cerevisiae activated spliceosome. Science 353(6306):1399-1405.

28. Yan C, Wan R, Bai R, Huang G, Shi Y (2016) Structure of a yeast activated spliceosome at $3.5 \AA$ resolution. Science 353(6302):904-911.

29. Semlow DR, Blanco MR, Walter NG, Staley JP (2016) Spliceosomal DEAH-Box ATPases Remodel Pre-mRNA to Activate Alternative Splice Sites. Cell 164(5):985-998.

30. Boneberg F, et al. (2019) Molecular mechanism of the RNA helicase DHX37 and its activation by UTP14 in ribosome biogenesis. RNA. doi:10.1261/rna.069609.118.

31. Hamann F, Enders M, Ficner R (2019) Structural basis for RNA translocation by DEAH-box ATPases. Nucleic Acids Res. doi:10.1093/nar/gkz150.

32. Kozlowski LP, Bujnicki JM (2012) MetaDisorder: a meta-server for the prediction of intrinsic disorder in proteins. BMC Bioinformatics 13:111.

33. Absmeier E, et al. (2015) A noncanonical PWI domain in the N-terminal helicase-associated region of the spliceosomal Brr2 protein. Acta Crystallogr D Biol Crystallogr 71(Pt 4):762-771.

34. Absmeier E, Becke C, Wollenhaupt J, Santos KF, Wahl MC (2017) Interplay of cis- and transregulatory mechanisms in the spliceosomal RNA helicase Brr2. Cell Cycle 16(1):100-112. 
Chapter 3: Structural analysis of the interaction of Spp2 G-patch with Prp2

35. Schmitt A, Hamann F, Neumann P, Ficner R (2018) Crystal structure of the spliceosomal DEAH-box ATPase Prp2. Acta Crystallogr D Struct Biol 74(Pt 7):643-654.

36. Tauchert MJ, Fourmann J-B, Christian H, Lührmann R, Ficner R (2016) Structural and functional analysis of the RNA helicase Prp43 from the thermophilic eukaryote Chaetomium thermophilum. Acta Crystallogr F Struct Biol Commun 72(Pt 2):112-120.

37. Tauchert MJ, Fourmann J-B, Lührmann R, Ficner R (2017) Structural insights into the mechanism of the DEAH-box RNA helicase Prp43. eLife 6:e21510.

38. Ulrich A, Wahl MC (2014) Structure and evolution of the spliceosomal peptidyl-prolyl cis-trans isomerase Cwc27. Acta Crystallogr D Biol Crystallogr 70(Pt 12):3110-3123.

39. Wishart DS, Sykes BD (1994) The 13C chemical-shift index: a simple method for the identification of protein secondary structure using 13C chemical-shift data. J Biomol NMR 4(2):171-180.

40. Marsh JA, Singh VK, Jia Z, Forman-Kay JD (2006) Sensitivity of secondary structure propensities to sequence differences between alpha- and gamma-synuclein: implications for fibrillation. Protein Sci 15(12):2795-2804.

41. Wu H-Y, Shen X-H, Ni R-S, Qian X-Y, Gao X (2009) [Expression of E-cadherin and uPA and their prognostic value in carcinoma of human larynx.]. Zhonghua Er Bi Yan Hou Tou Jing Wai Ke Za Zhi 44(12):1024-1028.

42. Tsai R-T, et al. (2007) Dynamic interactions of Ntr1-Ntr2 with Prp43 and with U5 govern the recruitment of Prp43 to mediate spliceosome disassembly. Mol Cell Biol 27(23):8027-8037.

43. Fourmann J-B, et al. (2016) The target of the DEAH-box NTP triphosphatase Prp43 in Saccharomyces cerevisiae spliceosomes is the U2 snRNP-intron interaction. eLife 5:e15564.

44. Walbott $\mathrm{H}$, et al. (2010) Prp43p contains a processive helicase structural architecture with a specific regulatory domain. EMBO J 29(13):2194-2204.

45. Haselbach D, et al. (2018) Structure and Conformational Dynamics of the Human Spliceosomal Bact Complex. Cell 172(3):454-464.e11.

46. Zhang X, et al. (2018) Structure of the human activated spliceosome in three conformational states. Cell Res 28(3):307-322.

47. Ferrage F, Cowburn D, Ghose R (2009) Accurate sampling of high-frequency motions in proteins by steady-state $(15) \mathrm{N}-\{(1) \mathrm{H}\}$ nuclear Overhauser effect measurements in the presence of cross-correlated relaxation. J Am Chem Soc 131(17):6048-6049.

48. Delaglio F, et al. (1995) NMRPipe: a multidimensional spectral processing system based on UNIX pipes. J Biomol NMR 6(3):277-293.

49. Goddard TD, Kneller DG (2009) SPARKY3.

50. Sattler M, Schleucher J, Griesinger C (1999) Heteronuclear multidimensional NMR experiments for the structure determination of proteins in solution employing pulsed field gradients. Progress in Nuclear Magnetic Resonance Spectroscopy 34(2):93-158. 
Chapter 3: Structural analysis of the interaction of Spp2 G-patch with Prp2

51. Vranken WF, et al. (2005) The CCPN data model for NMR spectroscopy: development of a software pipeline. Proteins 59(4):687-696.

52. Kabsch W (2010) XDS. Acta Crystallogr D Biol Crystallogr 66(Pt 2):125-132.

53. McCoy AJ, et al. (2007) Phaser crystallographic software. J Appl Crystallogr 40(Pt 4):658-674.

54. Adams PD, et al. (2010) PHENIX: a comprehensive Python-based system for macromolecular structure solution. Acta Crystallogr Sect D-Biol Crystallogr 66:213-221.

55. Emsley P, Lohkamp B, Scott WG, Cowtan K (2010) Features and development of Coot. Acta Crystallogr D Biol Crystallogr 66(Pt 4):486-501.

56. Chen VB, et al. (2010) MolProbity: all-atom structure validation for macromolecular crystallography. Acta Crystallogr D Biol Crystallogr 66(Pt 1):12-21.

57. Schmidt C, Urlaub H (2009) iTRAQ-labeling of in-gel digested proteins for relative quantification. Methods Mol Biol 564:207-226.

58. Houtman JC, et al. (2007) Studying multisite binary and ternary protein interactions by global analysis of isothermal titration calorimetry data in SEDPHAT: application to adaptor protein complexes in cell signaling. Protein Sci 16(1):30-42.

59. Keller S, et al. (2012) High-precision isothermal titration calorimetry with automated peak-shape analysis. Anal Chem 84(11):5066-5073.

60. Brautigam CA (2015) Fitting two- and three-site binding models to isothermal titration calorimetric data. Methods 76:124-136. 
Chapter 3: Structural analysis of the interaction of Spp2 G-patch with Prp2
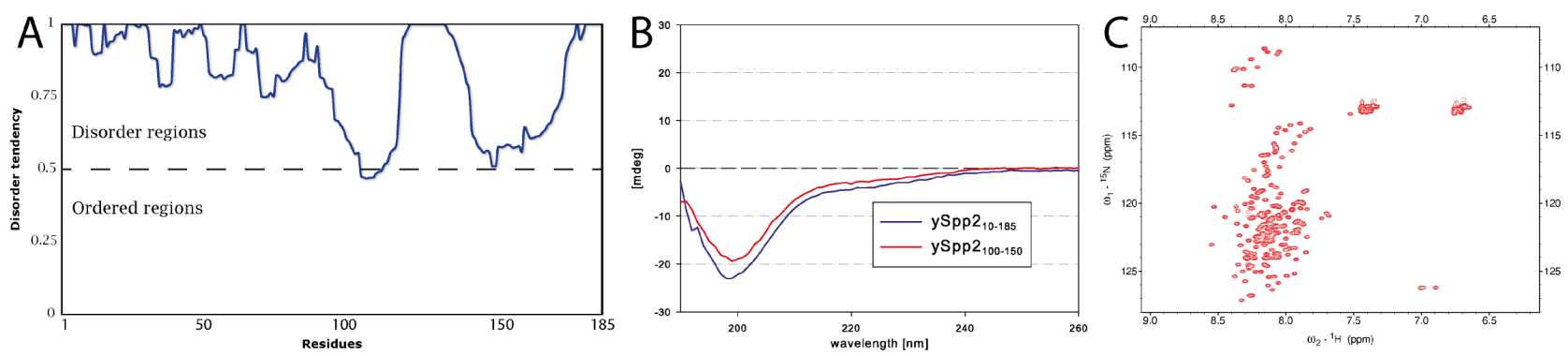

Figure 1: Disorder and secondary structure analysis of yeast Spp2 in solution

(A) Analysis of the scSpp2 amino acid sequence by Genesilico MetaDisorder prediction server . The probability to form disordered regions (y-axis) is shown with respect to the residue number (x-axis). (B) Circular dichroism spectra of scSpp2 ${ }_{10-185}$ (blue line) and scSpp2 ${ }_{100-150}$ (red line). The circular dichroism in mdeg (y-axis) is shown with respect to the wavelength (x-axis). (C) Two-dimensional ${ }^{1} \mathrm{H}-{ }^{15} \mathrm{~N}$ HSQC-spectrum of scSpp2 $10-185$ at $288 \mathrm{~K}$ and pH 6.5 . 
Chapter 3: Structural analysis of the interaction of Spp2 G-patch with Prp2

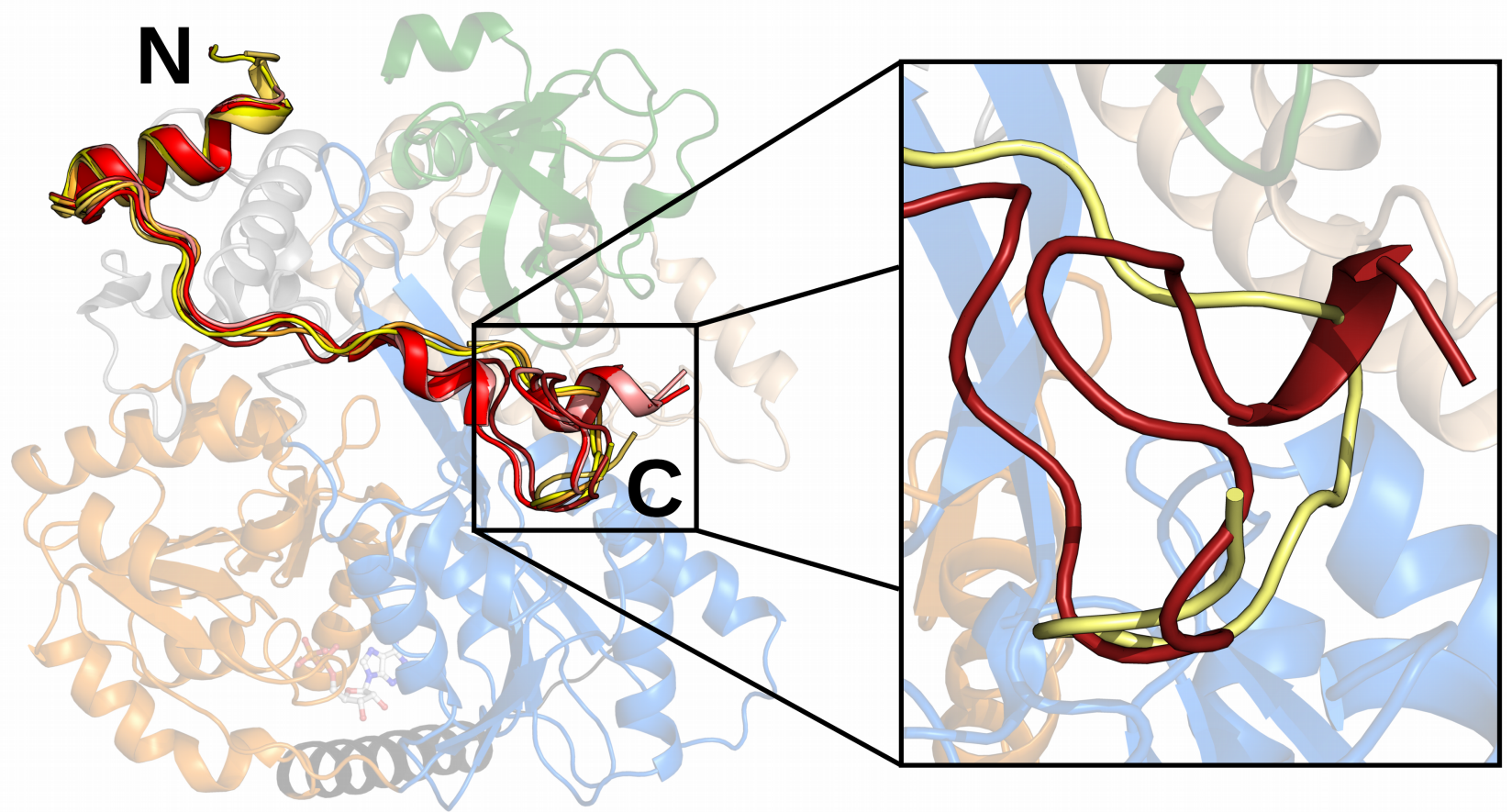

\section{Prp2}

Spp2 - conformation $1 \quad$ Spp2 - conformation 2 211 \begin{tabular}{l|lllllll}
\hline CF1 & CF5-1 & CF5-2 & 254 \\
\hline
\end{tabular}

Figure 2: Structural overview of the ctSpp2 G-patch bound to ctPrp2

The model of the ctPrp2-ctSpp2 $211-254$ complex is depicted as a cartoon model and ctPrp2 is displayed semitransparent. N-terminual residues (270-296) of ctPrp2 are shown in black, the RecA1 domain (297-476) in orange, the RecA2 domain (477-653) in blue, the winged-helix domain (WH; 654-721) in gray, the helixbundle domain (HB; 722-840) in wheat and the oligonucleotide-binding domain (OB; 841-921) in green. Two alternative conformations of the ctSpp2 G-patch were found in complex structures obtained from five crystal forms (CF). ctSpp2 $2_{211-254}$ molecules exhibiting conformation 1 are depicted in different shades of red, whereas molecules belonging to conformation 2 are displayed in different shades of yellow. The right panel shows a zoomed in view of the alternative conformations at the C-terminal end of the G-patch with one representative for each conformation. 
ctSpp2 ${ }^{201}$ PQLEDYKAVPVDDFGENLLRSFGWD. GKMRG . . . . . . KVKEVK . . . RY. ANLAGLGARNVKEAED ${ }^{254}$ SCSpp2 92 VTEKEYNEVPVEEFGDALLRGMGWESDSEQDSKGDKTQSRNKDVSNVSQIH. PDGLGIGAKLNKAINV 158 hsSpp2 ${ }^{154}$ PEEANYEAVPVEAYGLAMLRGMGWKPGEGIGRTFNQ . . . VVKPRV . . NSLR. PKGLGLGANLTEAQAL ${ }^{215}$ SCNtr1 ${ }^{51}$ NAPTISKLTKTYGIGAKLLSSMGYVAGKGLGKDGS. G. . ITTPIE . TQSRPMHNAGLGMFSNTNSSN 113 SCGnO1 ${ }^{15}$ DPRNTAWSNDTSRFGHQFLEKFGWKPGMGLGLSPMNS . NTSHIK . . VSIK. DDNVGLGAKLKRKDKK 77 SCPfa1 ${ }^{710}$ IVGQNAPEIGNENIGRRMLEKLGWKSGEGLGIQGNKG. . ISEPIF . AKIK . KNRSGLRHSES 767

B

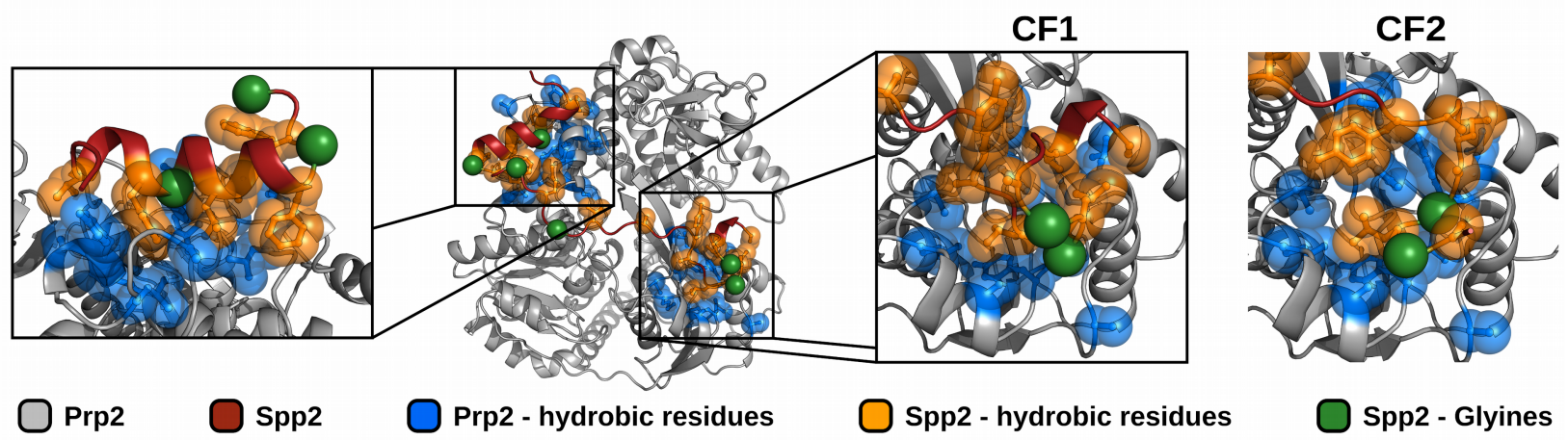

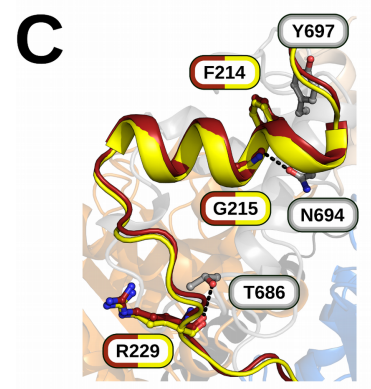

Spp2 - CF1

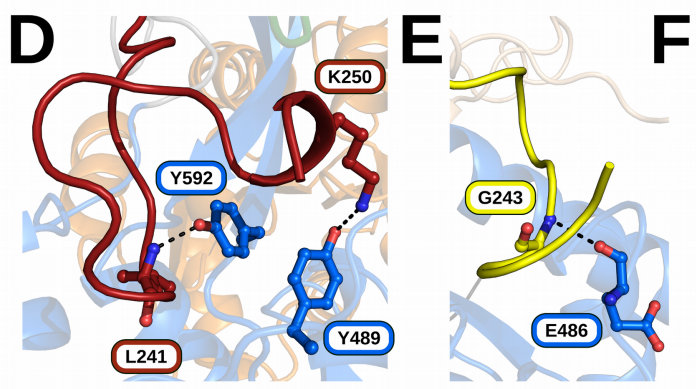

Spp2 - CF2

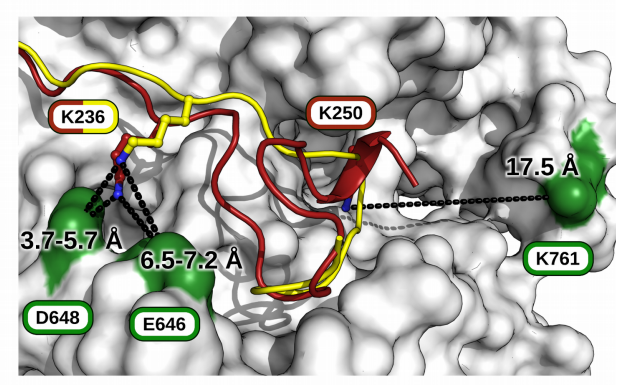

Prp2 Crosslinks

Figure 3: ctPrp2-ctSpp2 ${ }_{211-254}$ interactions

(A) Sequence alignment of the G-patch domains of Spp2 from C. thermophilum, S. cerevisiae and $H$. sapiens together with Ntr1, Gno1 and Pfa1 from S. cerevisiae. Conserved hydrophobic residues are highlighted in yellow whereas glycine residues are shown in red. Secondary structure elements present in either of the five crystal forms are displayed on top of the corresponding segment of the sequence. The $\mathrm{N}$ terminal amphipathic helix as well as a hydrophobic stretch at the C-terminal end are highly conserved. (B) Overview of hydrophobic interactions between ctSpp2 $2_{21-254}$ and ctPrp2. Hydrophobic residues of ctSpp2 are shown in orange, while hydrophobic residues of ctPrp2 within $8 \AA$ of the conserved ctSpp2 $211-254$ hydrophobic residues are displayed in blue. Glycine residues of ctSpp2 $2_{211-254}$ are highlighted as green spheres. (C) Additionally to the hydrophobic interactions, the N-terminal part of the G-patch is only involved in two further

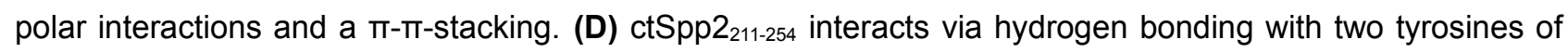
ctPrp2 in conformation 1. (E) In conformation $2 \mathrm{ctSpp2} 211-254$ is only involved in one polar interaction with ctPrp2. (F) Residue identified to cross-link to the lysines K236 and K250 of ctSpp2 ${ }_{211-254}$ are shown as sticks and the cross-linked residues on ctPrp2 are shown in green. 
Chapter 3: Structural analysis of the interaction of Spp2 G-patch with Prp2
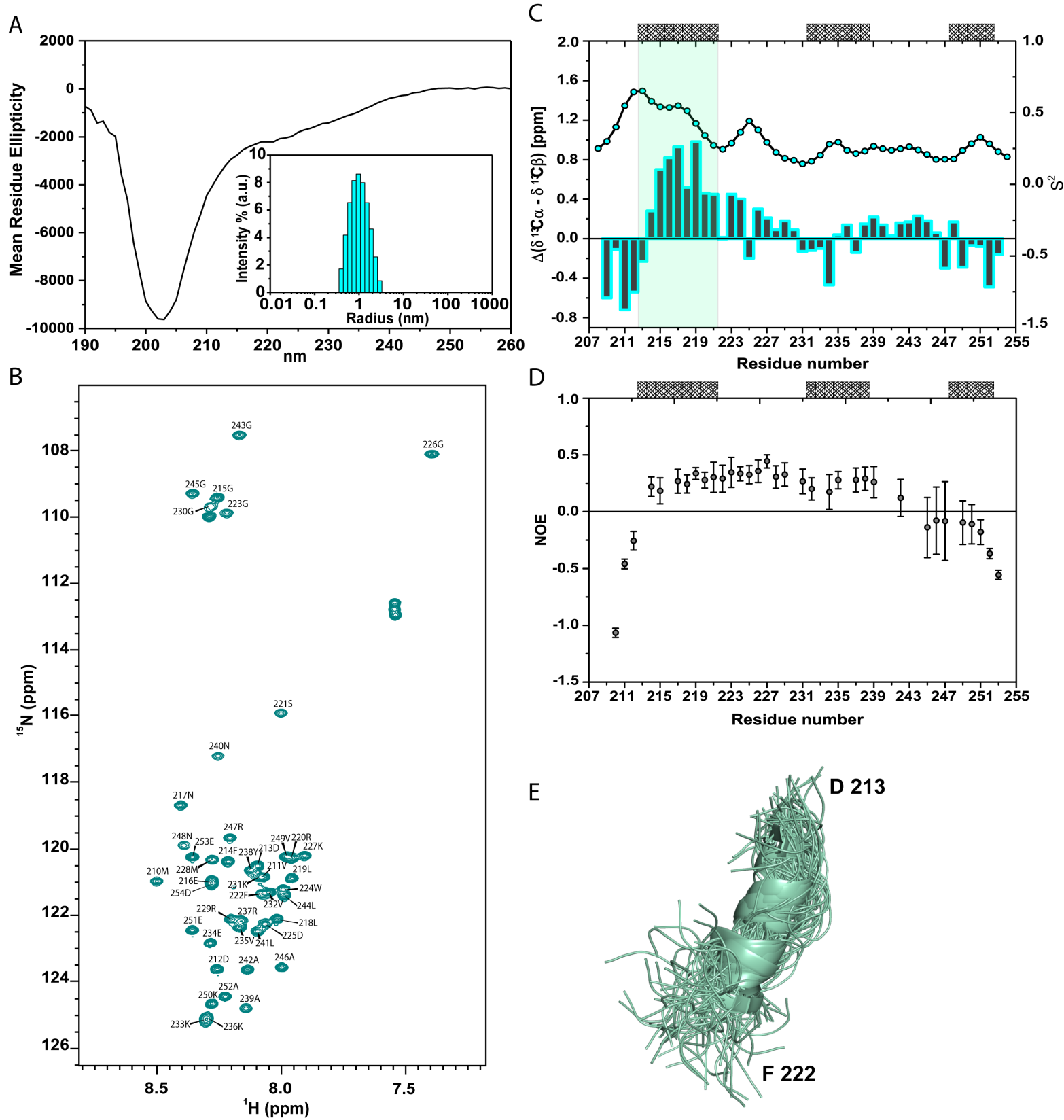

Figure 4: Transient helical conformations in the G-patch of ctSpp2 prior to complex formation.

(A) Far-UV CD spectrum (mean residue ellipticity vs wavelength in $\mathrm{nm}$ ) of ctSpp2 $2_{208-254}$. The inset shows the results of DLS measurements, demonstrating that ctSpp2 $208-254$ is predominantly monomeric in solution. (B) Two-dimensional ${ }^{1} \mathrm{H}-{ }^{15} \mathrm{~N}-\mathrm{HSQC}$ spectrum of ctSpp2 $208-254$. The sequence-specific assignment of the backbone resonances is indicated. (C) Residue-specific $\Delta C^{\alpha}-\Delta C^{\beta}$ secondary chemical shifts of ctSpp2 $208-254$ together with $S^{2}$ parameters derived by TALOS+. Positive values of $\Delta C^{\alpha}-\Delta C^{\beta}$ indicate propensity for $\alpha$-helical conformations, while negative values indicate the propensity to form extended structure. The position of $\alpha$ helices observed in ctSpp $2_{208-254}$ when in complex with Prp2 are shown on top. (D) Heteronuclear steadystate $\left\{{ }^{1} \mathrm{H},{ }^{15} \mathrm{~N}\right\}-\mathrm{NOE}$ as a function of residue number. (E) Ensemble of $\alpha$-helical conformations populated by residues D213-F221 of ctSpp2 $208-254$. 

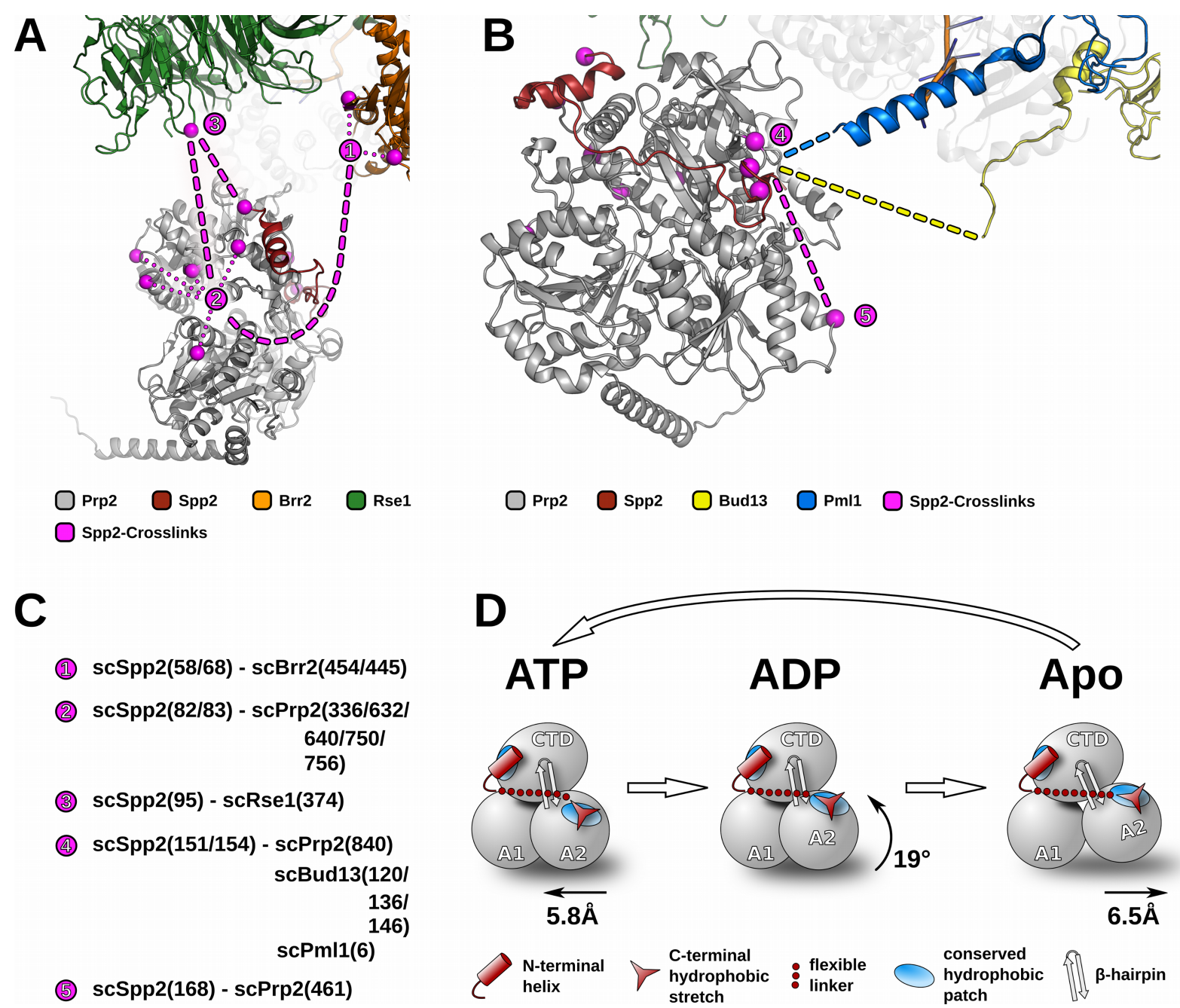

Figure 5: Spp2 in the spliceosome and its conformational adaptability to different DEAH-box ATPase conformations

Using a combination of the ctPrp2-ctSpp2 ${ }_{211-254}$ complex structure together with the cryo-EM and cross-link data from Rauhut et al. (2016), it can be estimated how Spp2 interacts with the spliceosome. All spliceosomal factors are depicted as cartoon models and cross-links of Spp2 are highlight as pink spheres. The estimated path of Spp2 is displayed as a pink dashed line and main contact sites are numbered starting from the most N-terminal cross-linked residue. (A) N-terminally from the G-patch Spp2 cross-links with Brr2 and Rse1, as well as with Prp2. (B) The C-terminal end of the Spp2 G-patch cross-links with the OB-fold domain of Prp2 and parts of Bud13 and Pml1. Cross-linked regions of Bud13 and Pml1 are not part of the cryo-EM model and are symbolically depicted as dashed lines. C-terminally from the G-patch Spp2 contacts another part of the RecA2 domain. (C) Overview of Spp2 cross-links numbered as in A and B. (D) Schematic representation of the catalytic states of Prp2 during one translocation cycle. The RecA2 domain is the most mobile domain during this process and due to the versatile conformations of the conserved C-terminal stretch together with the flexible linker region the Spp2 G-patch is able to adapt to the individual conformations of the RecA2 domain. 
Chapter 3: Structural analysis of the interaction of Spp2 G-patch with Prp2

Table 1. Data collection and refinement statistics.

\begin{tabular}{|c|c|c|c|c|c|}
\hline Data collection & CF1 & CF2 & CF3 & CF4 & CF5 \\
\hline Space group & $\mathrm{P} 2{ }_{1} 2_{1} 2_{1}$ & $\mathrm{P} 2{ }_{1} 2_{1} 2_{1}$ & $\mathrm{P} 2{ }_{1} 2_{1} 2_{1}$ & $P 2_{1}$ & $\mathrm{P} 2_{1} 2_{1} 2_{1}$ \\
\hline $\mathrm{a}(\AA)$ & 64.3 & 65.9 & 66.7 & 69.4 & 74.7 \\
\hline $\mathrm{b}(\AA)$ & 75.5 & 77.4 & 95.3 & 72.6 & 113.7 \\
\hline$c(\AA)$ & 154.4 & 148.9 & 124.8 & 70.3 & 191.7 \\
\hline$\beta\left(^{\circ}\right)$ & 90.0 & 90.0 & 90.0 & 93.3 & 90.0 \\
\hline X-ray source & $\begin{array}{l}\text { P13, PETRA } \\
\text { III, DESY }\end{array}$ & $\begin{array}{l}\text { P13, PETRA } \\
\text { III, DESY }\end{array}$ & $\begin{array}{l}\text { P14, PETRA } \\
\text { III, DESY }\end{array}$ & $\begin{array}{l}\text { P14, PETRA } \\
\text { III, DESY }\end{array}$ & $\begin{array}{l}\text { P13, PETRA } \\
\text { III, DESY }\end{array}$ \\
\hline $\begin{array}{l}\text { Resolution range } \\
(\AA)\end{array}$ & $\begin{array}{l}77.22-1.95 \\
(2.05-1.95)\end{array}$ & $\begin{array}{l}74.45-1.85 \\
(1.95-1.85)\end{array}$ & $\begin{array}{l}45.55-2.10 \\
(2.20-2.10)\end{array}$ & $\begin{array}{l}70.15-2.50 \\
(2.60-2.50)\end{array}$ & $\begin{array}{l}48.90-2.60 \\
(2.71-2.60)\end{array}$ \\
\hline $\begin{array}{l}\text { No. of unique } \\
\text { reflections }\end{array}$ & 55604 & 65564 & 46827 & 23946 & 50939 \\
\hline Completeness (\%) & $100.0(99.9)$ & $99.7(99.8)$ & $99.4(99.4)$ & $98.5(98.7)$ & $99.6(99.7)$ \\
\hline $\mathrm{R}_{\text {merge }}(\%)$ & 7.4 (133.5) & $6.6(109.9)$ & $5.3(114.4)$ & $7.8(66.1)$ & $6.9(93.0)$ \\
\hline Average $\mathrm{I} / \sigma(\mathrm{I})$ & $15.97(2.08)$ & $11.45(1.84)$ & $14.83(1.58)$ & $12.16(1.92)$ & $17.33(1.83)$ \\
\hline Redundancy & 9.37 (9.75) & $4.55(4.64)$ & $4.54(4.65)$ & $3.16(3.07)$ & $5.04(5.10)$ \\
\hline $\mathrm{CC}_{1 / 2}$ & $99.9(81.0)$ & $99.8(67.8)$ & $99.9(63.3)$ & $99.7(79.3)$ & $99.9(72.6)$ \\
\hline Wilson $B\left(\AA^{2}\right)$ & 46.62 & 45.42 & 55.89 & 55.35 & 66.75 \\
\hline \multicolumn{6}{|l|}{ Refinement } \\
\hline Resolution $(\AA)$ & $\begin{array}{l}42.53-1.95 \\
(1.98-1.95)\end{array}$ & $\begin{array}{l}68.69-1.85 \\
(1.88-1.85)\end{array}$ & $\begin{array}{l}45.55-2.10 \\
(2.14-2.10)\end{array}$ & $\begin{array}{l}70.15-2.50 \\
(2.60-2.50)\end{array}$ & $\begin{array}{l}48.90-2.60 \\
(2.65-2.60)\end{array}$ \\
\hline No. of reflections & 55587 & 65546 & 46795 & 23930 & 50847 \\
\hline $\mathrm{R}_{\text {work }}(\%)$ & $20.00(32.3)$ & $19.79(34.9)$ & $22.41(34.1)$ & $20.81(32.4)$ & $22.22(38.1)$ \\
\hline $\mathrm{R}_{\text {free }}(\%)$ & $21.78(34.6)$ & $21.20(36.0)$ & $24.44(34.6)$ & $25.80(38.1)$ & $25.04(40.8)$ \\
\hline $\begin{array}{l}\text { Molecules per } \\
\text { asymmetric unit }\end{array}$ & 1 & 1 & 1 & 1 & 2 \\
\hline $\begin{array}{l}\text { Total number of } \\
\text { atoms }\end{array}$ & 5782 & 5850 & 5611 & 5022 & 10938 \\
\hline Protein residues & 700 & 694 & 688 & 656 & 1375 \\
\hline Water molecules & 190 & 319 & 192 & 4 & 116 \\
\hline \multicolumn{6}{|l|}{ r.m.s. deviations } \\
\hline Bond length $(\AA)$ & 0.004 & 0.011 & 0.009 & 0.009 & 0.004 \\
\hline Bond angles $\left({ }^{\circ}\right)$ & 0.66 & 1.23 & 1.06 & 1.06 & 0.54 \\
\hline $\begin{array}{l}\text { Mean B-factors } \\
\left(\AA^{2}\right)\end{array}$ & 54.35 & 49.22 & 61.67 & 61.61 & 78.70 \\
\hline \multicolumn{6}{|l|}{$\begin{array}{l}\text { Ramachandran } \\
\text { statistics }\end{array}$} \\
\hline favoured (\%) & 96.84 & 97.97 & 98.24 & 95.81 & 96.41 \\
\hline allowed (\%) & 3.02 & 2.03 & 1.76 & 3.73 & 3.37 \\
\hline outliers (\%) & 0.14 & 0.00 & 0.00 & 0.47 & 0.22 \\
\hline $\begin{array}{l}\text { PDB accession } \\
\text { code }\end{array}$ & 6rm8 & 6rm9 & 6rma & $6 r m b$ & 6rmc \\
\hline
\end{tabular}


Chapter 3: Structural analysis of the interaction of Spp2 G-patch with Prp2

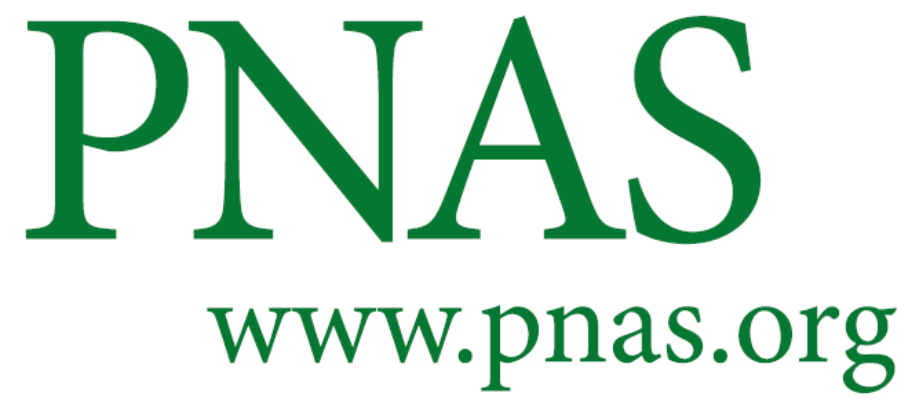

\section{Supplementary Information}

Structural analysis of the intrinsically disordered splicing factor Spp2 and its binding to the DEAH-box ATPase Prp2

Florian Hamann, Andreas Schmitt, Filippo Favretto, Romina Hofele, Piotr Neumann, ShengQi Xiang, Henning Urlaub, Markus Zweckstetter and Ralf Ficner

Ralf Ficner

Email: rficner@uni-goettingen.de

\section{This PDF file includes:}

Figs. S1 to S11 
Chapter 3: Structural analysis of the interaction of Spp2 G-patch with Prp2

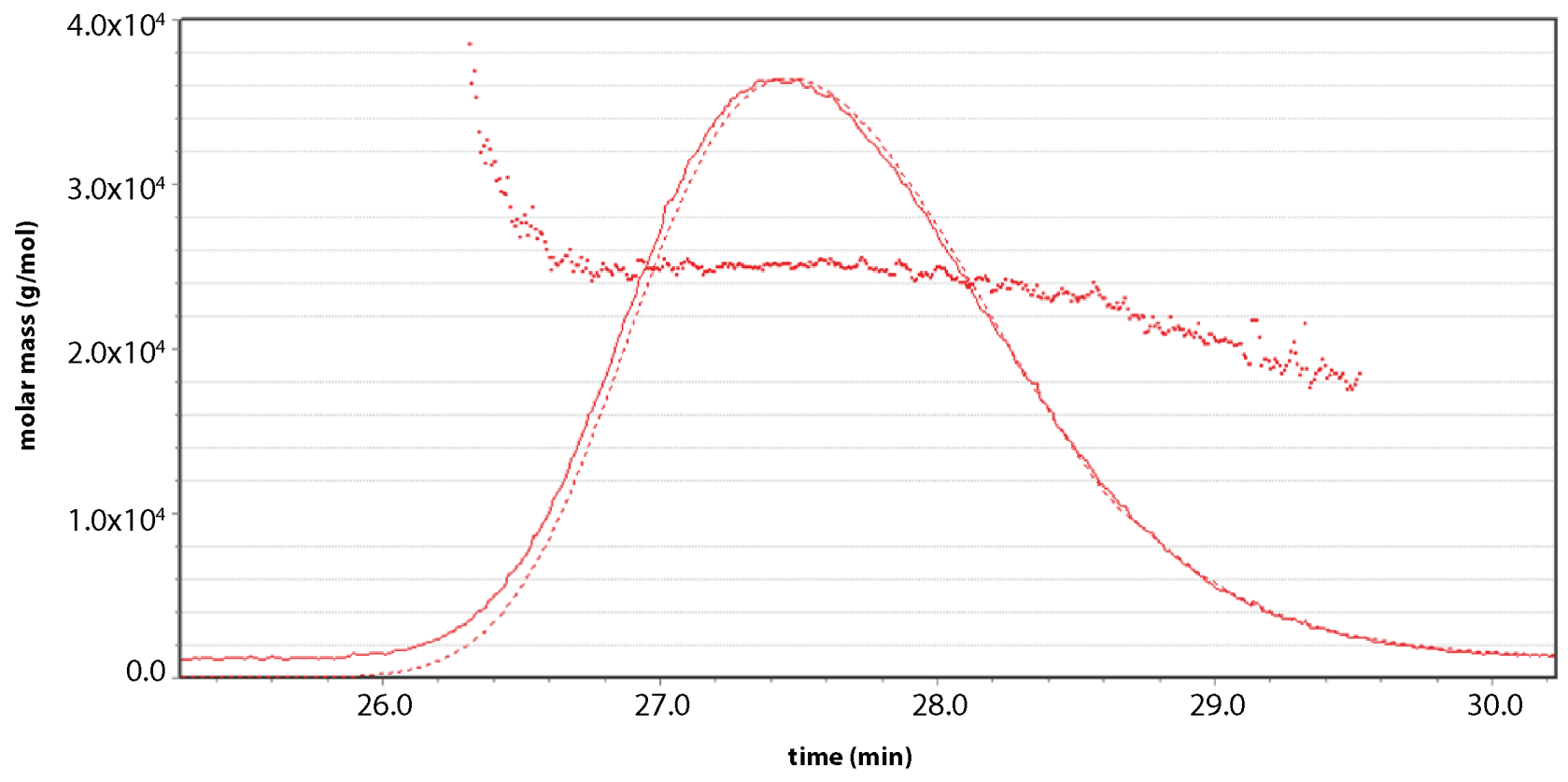

Fig. S1. MALS measurements of scSpp2 ${ }_{10-185}$

The determined molecular weight represented as dots is plotted against the time. UV-absorption and light scattering are shown as solid and dashed lines, respectively. The determined molecular weight of the protein within the UV Absorption peak is $2.453 \mathrm{e}+4 \mathrm{~g} / \mathrm{mol}$, corresponding to monomeric scSpp $2_{10-185}$. 
Chapter 3: Structural analysis of the interaction of Spp2 G-patch with Prp2
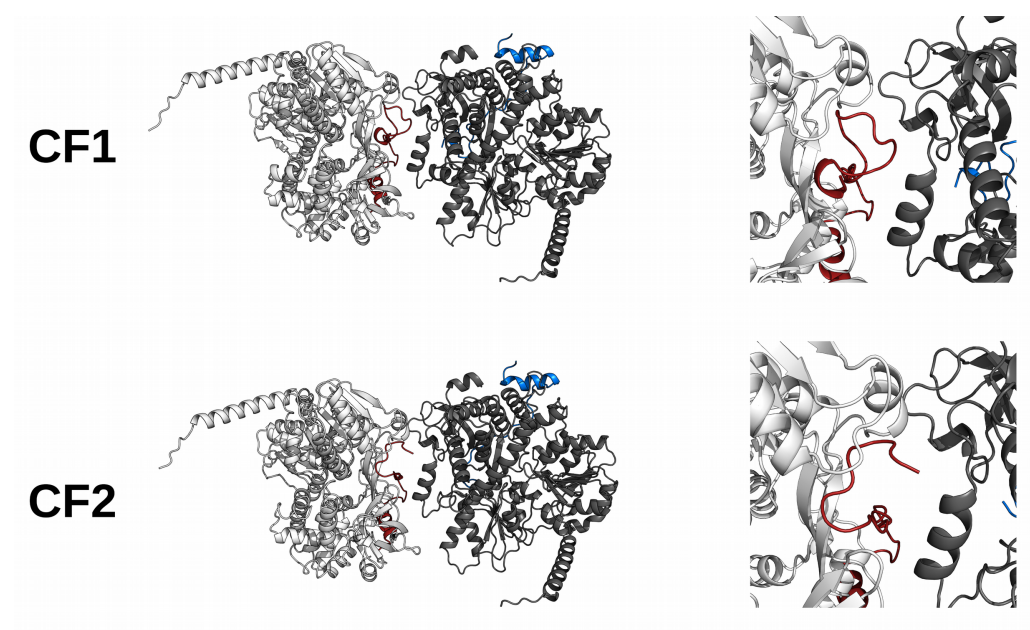

Fig. S2. Crystal contact overview of the C-terminal part of the ctSpp2 G-patch in the different crystal forms.

Prp2-Spp2 complexes are depicted as cartoon models. Prp2 is shown in

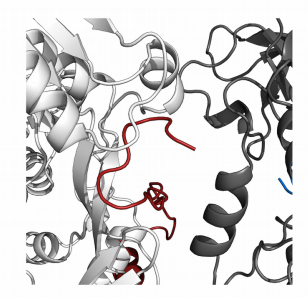
different shades of gray and Spp2 either in red or blue. The left panels show an overview of the closest symmetry-based complexes to the C-terminal part of the G-patch. The right panels display a closeup view of the C-terminal part of the G-

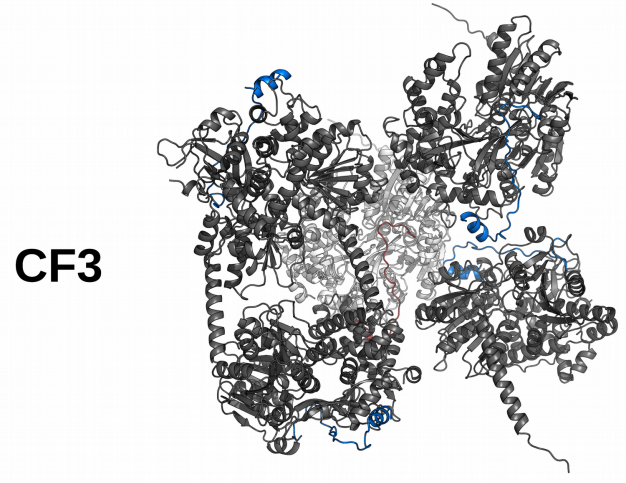
patch. CF1 and CF5 undergo crystal

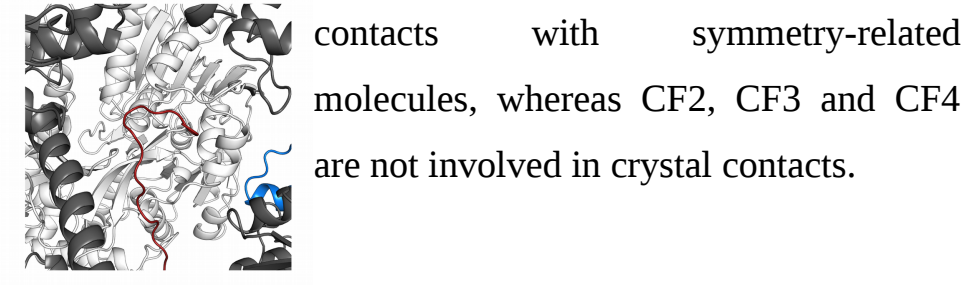

CF4
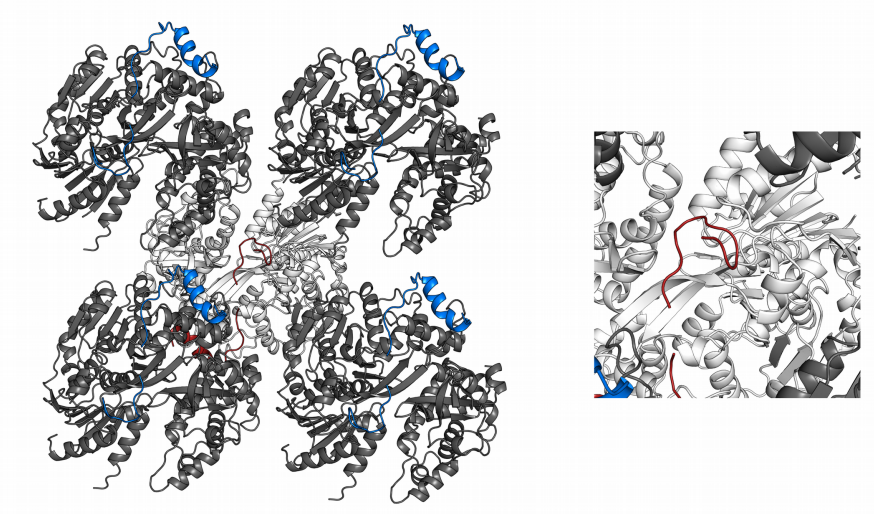

CF5
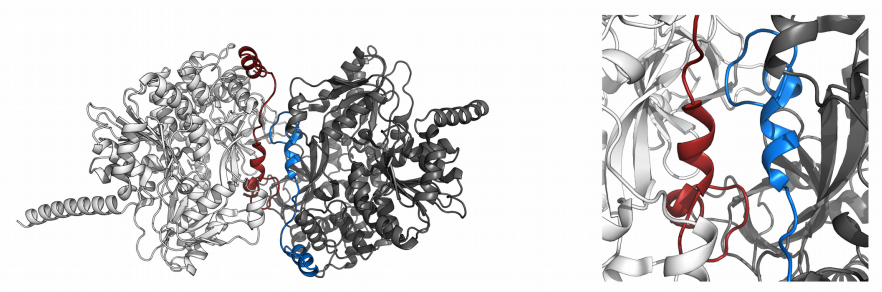

Prp2 Spp2 $\bigcirc$ Prp2 - symmetry

Spp2 - symmetry 
Chapter 3: Structural analysis of the interaction of Spp2 G-patch with Prp2
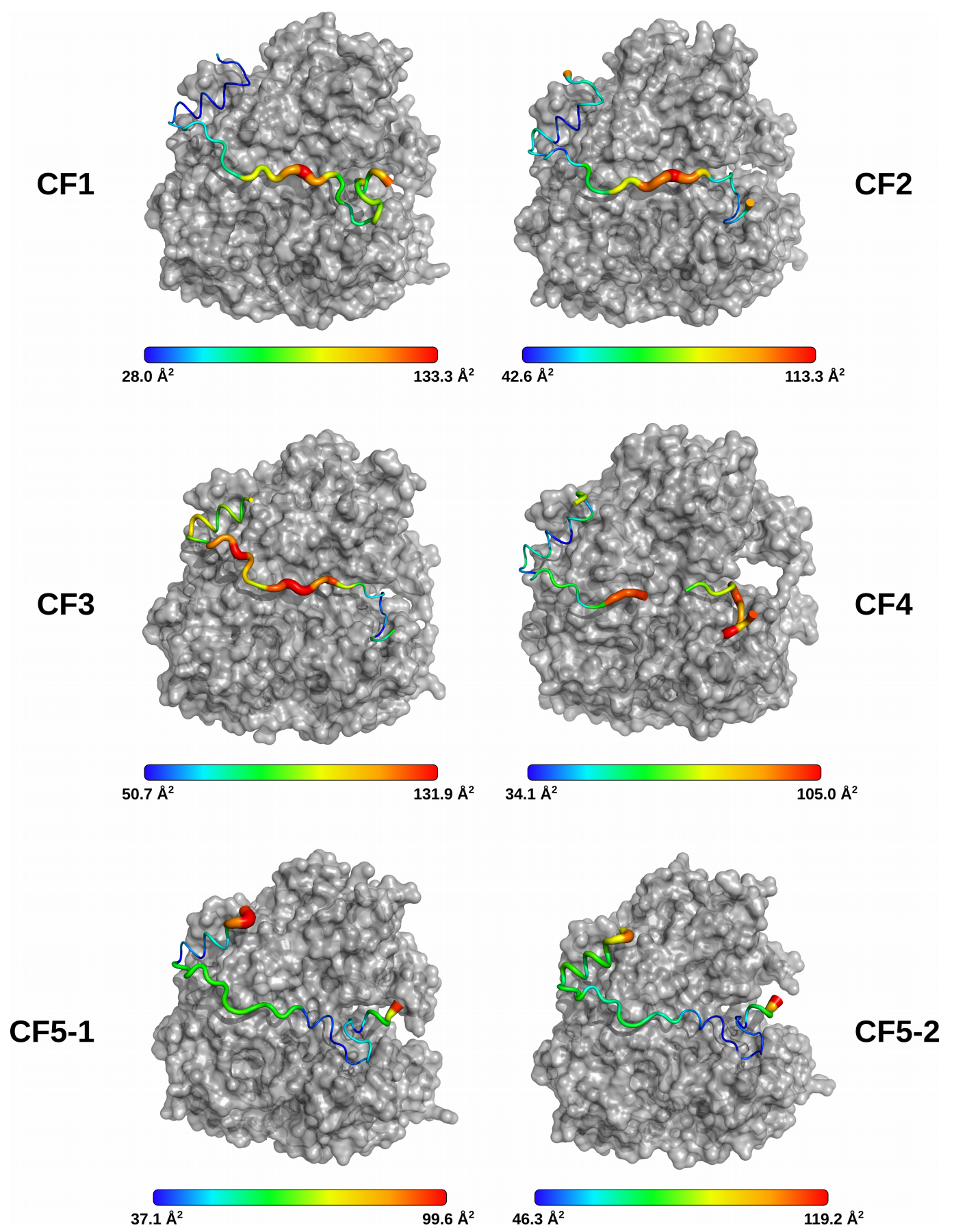

Fig S3. B-factors distribution of ctSpp2

The overview representation of the $B$-factors of ctSpp2 shows increased $B$-factor values for the linker connecting the $\mathrm{N}$-terminal $\alpha$-helix with the $\mathrm{C}$-terminal hydrophobic stretch, highlighting the flexibility of this region. $B$-factor values are not elevated in CF5 due to crystal contacts. 
Chapter 3: Structural analysis of the interaction of Spp2 G-patch with Prp2

A

\begin{tabular}{lccccc}
\cline { 2 - 5 } & \multicolumn{4}{c}{ Conformation 1 (CF1) } & \multicolumn{3}{c}{ Conformation 2 (CF2) } \\
\cline { 2 - 5 } & Phi & Psi & Phi & Psi \\
\hline Gly215 & -65.93 & -38.22 & -71.83 & -35.65 \\
\hline Gly223 & 95.43 & 11.15 & 93.09 & 12.24 \\
\hline Gly226 & 90.20 & 2.53 & 88.45 & -3.95 \\
\hline Gly230 & 87.30 & 149.42 & 70.61 & 163.06 \\
\hline Gly243 & 63.06 & 36.44 & -60.49 & 158.88 \\
\hline Gly245 & 82.9 & -142.61 & 76.28 & -5.27
\end{tabular}

General Case

B

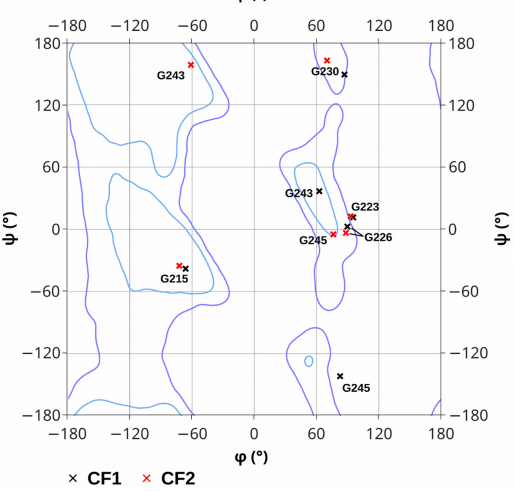

Glycine

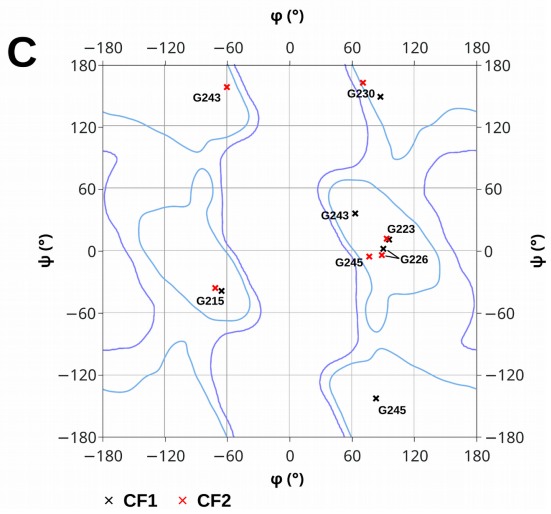

Fig. S4. Mainchain analysis of ctSpp2 glycines

(A) List of psi and phi angles of all glycines in the ctSpp2 G-patch. CF1 is used exemplary for conformation 1 and CF2 for conformation 2. (B) Ramachandran plot of ctSpp2 G-patch glycines depicted on favored (light blue) and allowed (dark blue) boundaries for all residues except glycine and proline. (C) Ramachandran plot of ctSpp2 G-patch glycines depicted on favored (light blue) and allowed (dark blue) boundaries for glycine. 
Chapter 3: Structural analysis of the interaction of Spp2 G-patch with Prp2
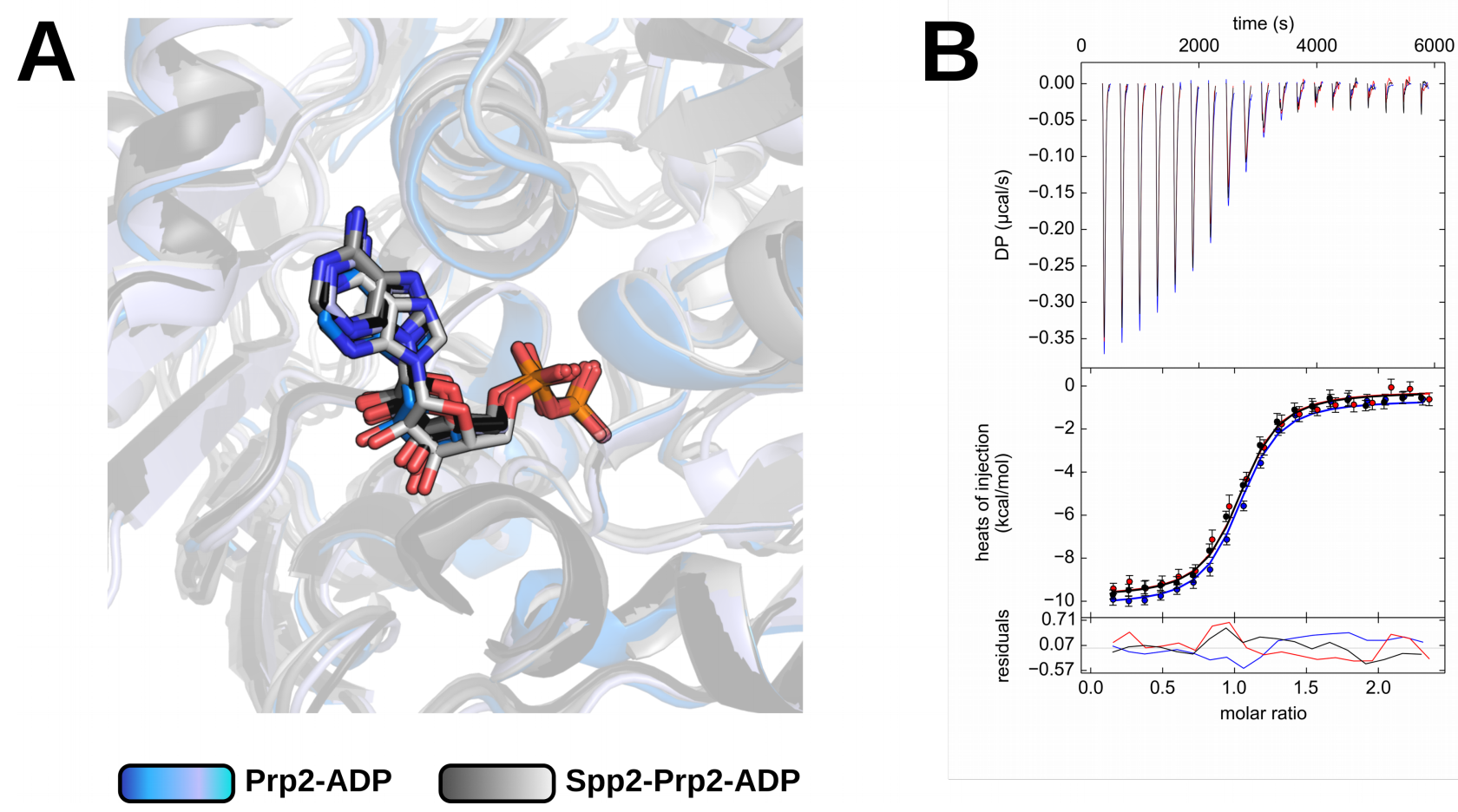

Fig. S5. ADP binding in Prp2-ADP and Spp2-Prp2-ADP complexes

(A) Prp2 is depicted as a semi-transparent cartoon model, the bound ADP is shown as a stick model. Prp2ADP complex structures are displayed in different shades of gray and Spp2-Prp2-ADP structures in different shades of blue. The nucleotide binding site of Prp2 is not altered by the binding of the Spp2 G-patch, leading to an almost identical binding of the ADP in all Prp2-ADP and Spp2-Prp2-ADP structures. (B) Isothermal titration calorimetry measurements of ADP binding to Prp2 in presence of a two fold molar excess of Spp2 G-patch. A dissociation constant $(K d)$ of $170 \mathrm{nM}$ was determined $(\mathrm{n}=1, \Delta \mathrm{H}=-10.06 \mathrm{kcal} / \mathrm{mol}, \Delta \mathrm{S}=$ $-3.57 \mathrm{cal} / \mathrm{mol} * \mathrm{~K})$. This value closely resembles the $K d$ of the ADP binding to Prp2 in absence of Spp2 Gpatch $(K d=179 \mathrm{nM})$, which was published by Schmitt el al. (2018). Individual triplicates are depicted in blue, black and red. The upper panel shows the thermograms, the middle panel shows the individual fits with the correspondent residual in the lower panel. 
Chapter 3: Structural analysis of the interaction of Spp2 G-patch with Prp2

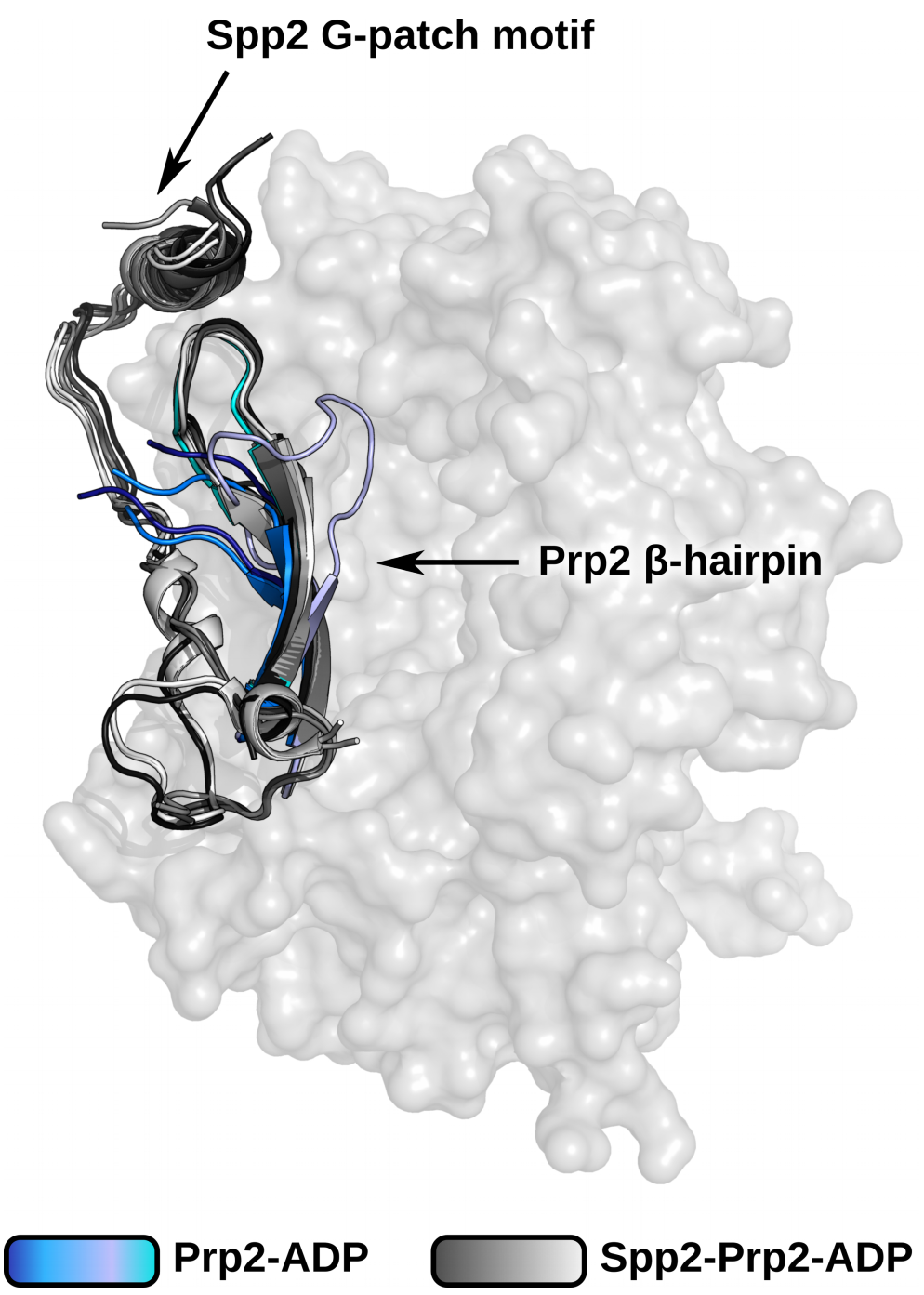

Fig. S6. Binding of Spp2 stabilized $\beta$-hairpin conformation

The overall structure of Prp2 is depicted as a semi-transparent surface model. The OB-fold domain was omitted for clarity reasons. Spp2 G-patch and the $\beta$-hairpin are displayed as cartoon models. Cartoon models shown in different shades of blue correspond to Prp2-ADP complex structures and models in different shades of gray represent Spp2-Prp2-ADP structures. While the $\beta$-hairpin exhibits multiple conformations in the different Prp2-ADP complex structures, the binding of Spp2 seems to stabilize one of these conformations in all Prp2-Spp2-ADP structures. 
Chapter 3: Structural analysis of the interaction of Spp2 G-patch with Prp2
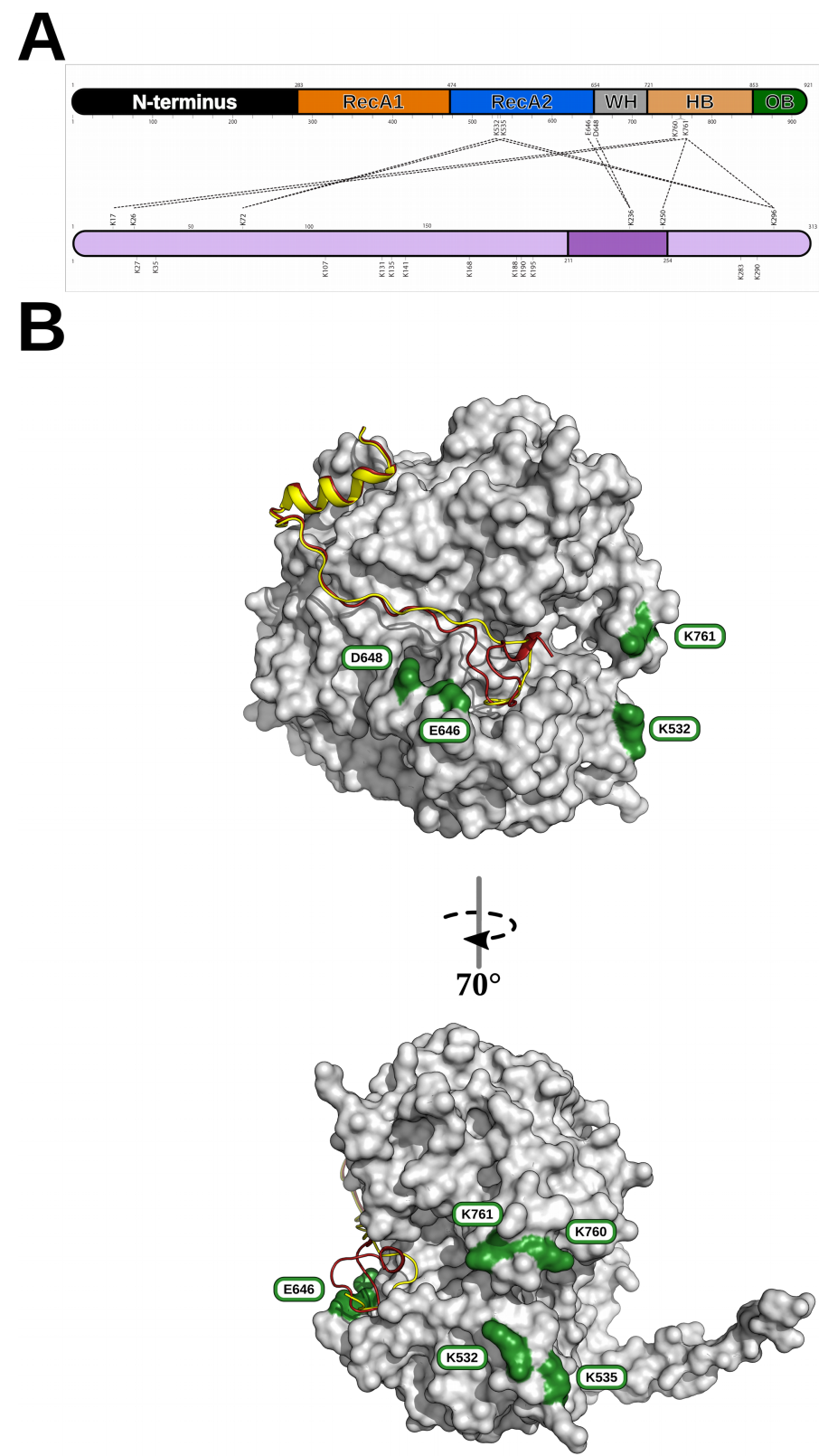

Fig. S7. Overview of cross-links between ctSpp2 and ctPrp2

(A) Map of ctSpp2-ctPrp2 cross-links. ctPrp2 is represented as the upper with coloring as in Figure 2 and ctSpp2 as the lower bar (length of bar not up to scale). Intermolecular cross-links are indicated by dashed lines between the corresponding residues. (B) ctPrp2 is depicted as a surface model in gray and the two alternative ctSpp2 conformations are displayed as cartoon models in red (conformation 1) and yellow (conformation 2). Cross-linked residues of ctPrp2 are highlighted in green and labeled accordingly. 

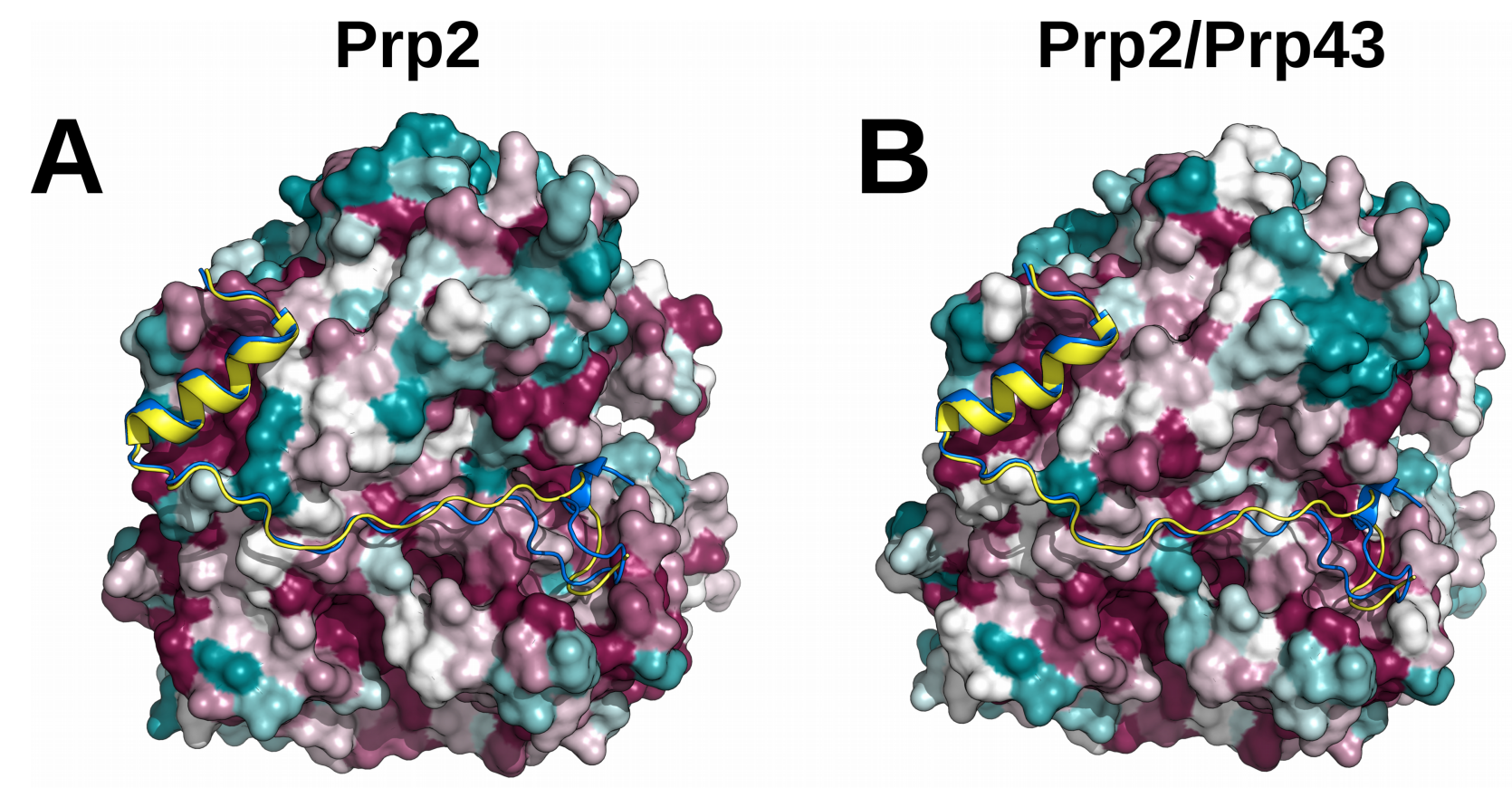

Fig. S8. Sequence conservation of Prp2 and Prp43

(A) The surface model of Prp2 is colored by sequence conservation using an alignment of Prp2 from $C$. thermophilum, S. cerevisiae, H. sapiens, M. musculus, $N$. crassa and D. rerio, with conserved and variable residues in purple and green, respectively. Residues of Prp2 interacting with the N-terminal $\alpha$-helix and the C-terminal end of ctSpp2 G-patch are conserved. (B) The surface model of Prp2 is colored by sequence conservation using an alignment of Prp2 from C. thermophilum, S. cerevisiae and H. sapiens together with Prp43 from the same organisms. Prp43 shows the same conservation for the interaction sites with the Nterminal and C-terminal ends. 
Chapter 3: Structural analysis of the interaction of Spp2 G-patch with Prp2

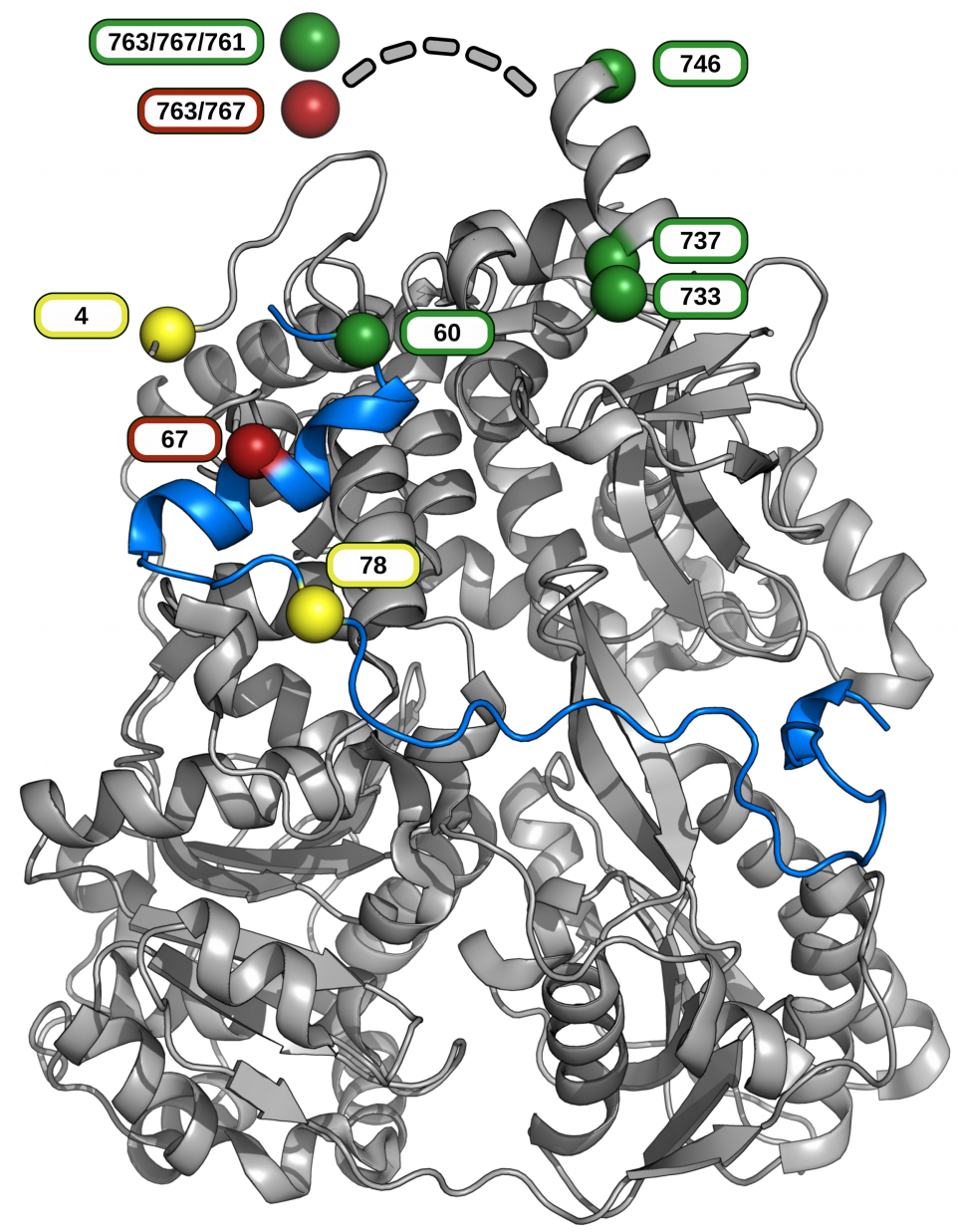

Fig. S9. Overview of scNtr1 and scPrp43 cross-links

scPrp43 is depicted as a gray cartoon model and Ntr1 was modeled as a blue cartoon model onto the scPrp43 structure assuming a similar binding as Spp2 to Prp2. Cross-link pairs identified by Christian et al. (2014) between Ntr1 and Prp43 are colored in the same color and labeled accordingly. The found cross-links are in a feasible distance for an interaction mode of Ntr1 with Prp43 similar to the Spp2-Prp2 complex. 
Chapter 3: Structural analysis of the interaction of Spp2 G-patch with Prp2

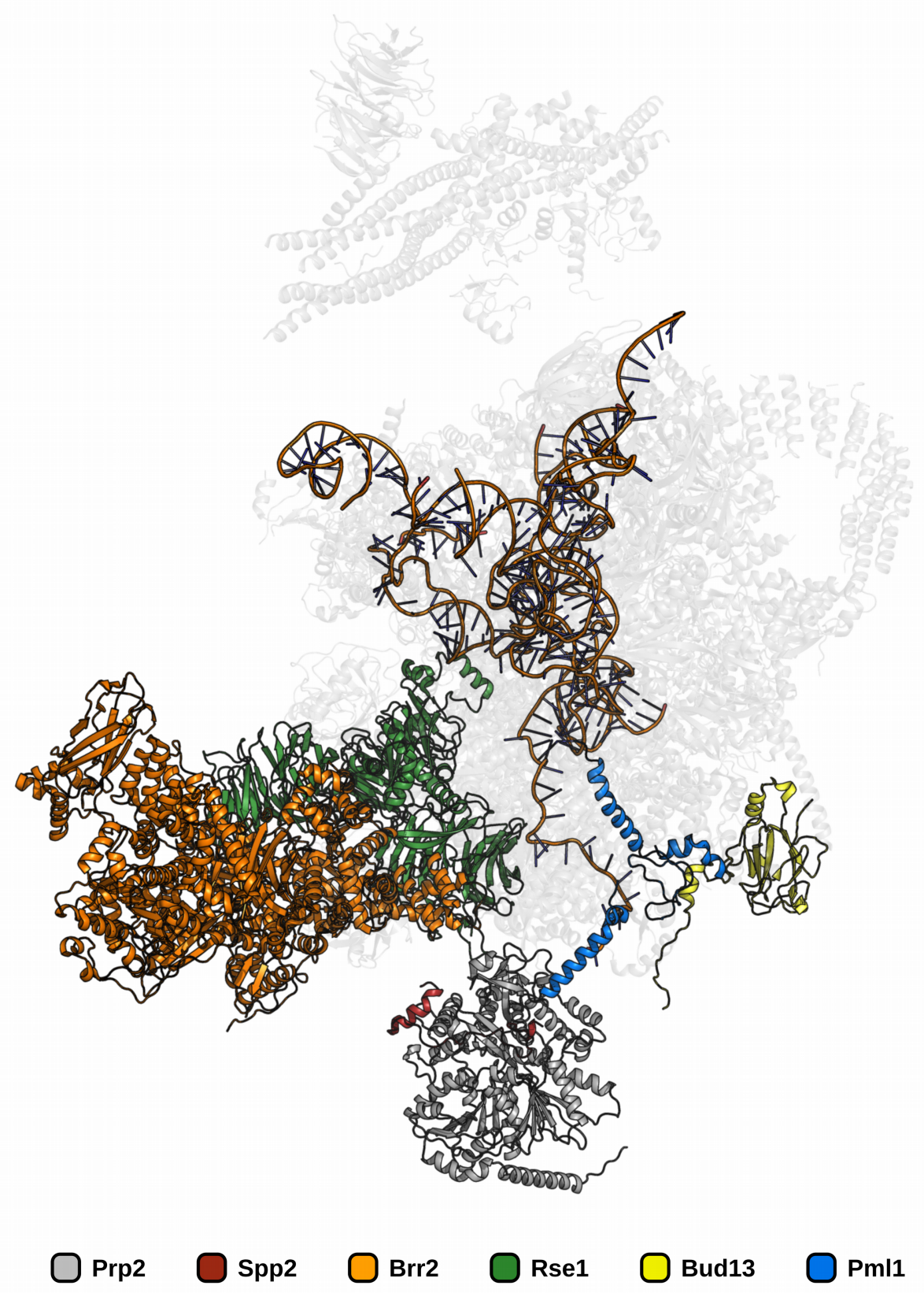

Fig. S10. Prp2-Spp2 complex in the spliceosomal $\mathrm{B}^{\text {act }}$ complex

The $\mathrm{B}^{\text {act }}$ spliceosome is depicted as a cartoon model. Spliceosomal factors not found to cross-link with Prp2 or Spp2 by Rauhut et al. (2016) are shown as gray semi-transparent cartoon models. The Prp2-Spp2 complex structure was superimposed on the scPrp2 model of the $\mathrm{B}^{\text {act }}$ complex in order to estimate the position of the Spp2 G-patch in this spliceosomal complex. 
Chapter 3: Structural analysis of the interaction of Spp2 G-patch with Prp2

\section{ATP}

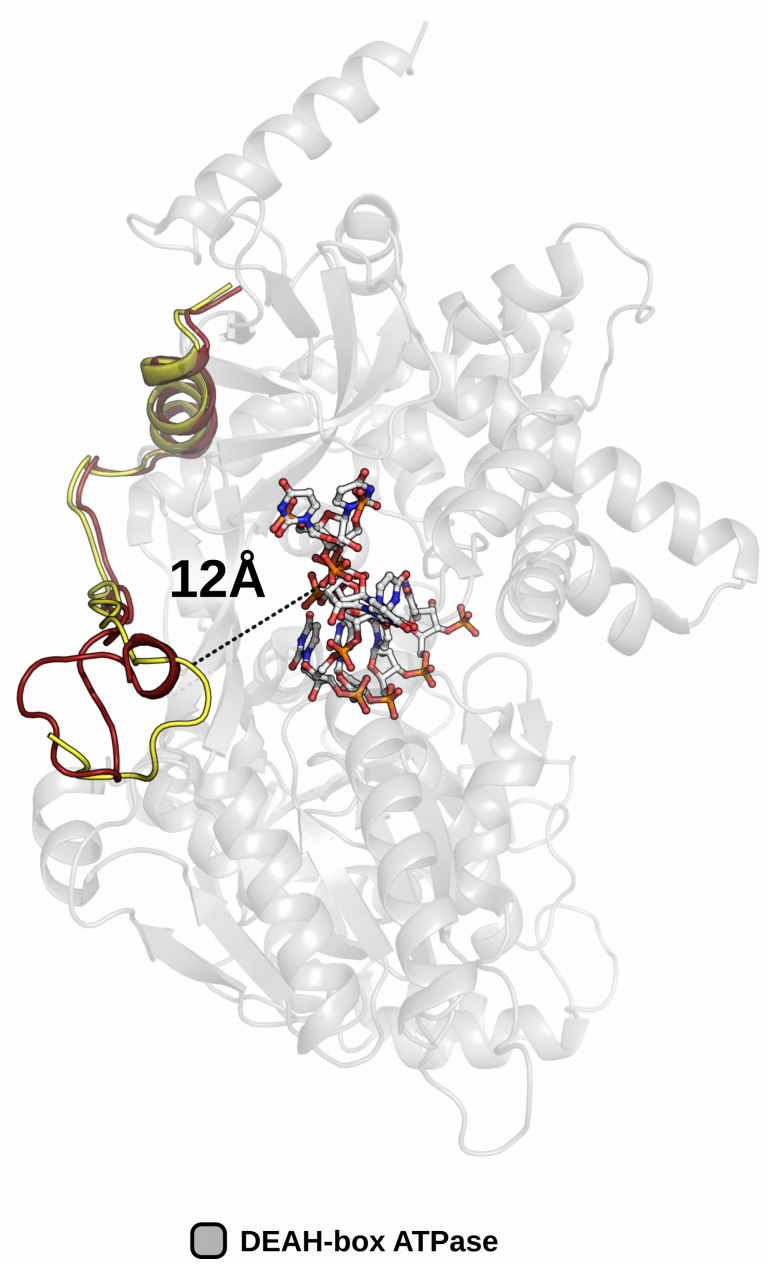

Apo

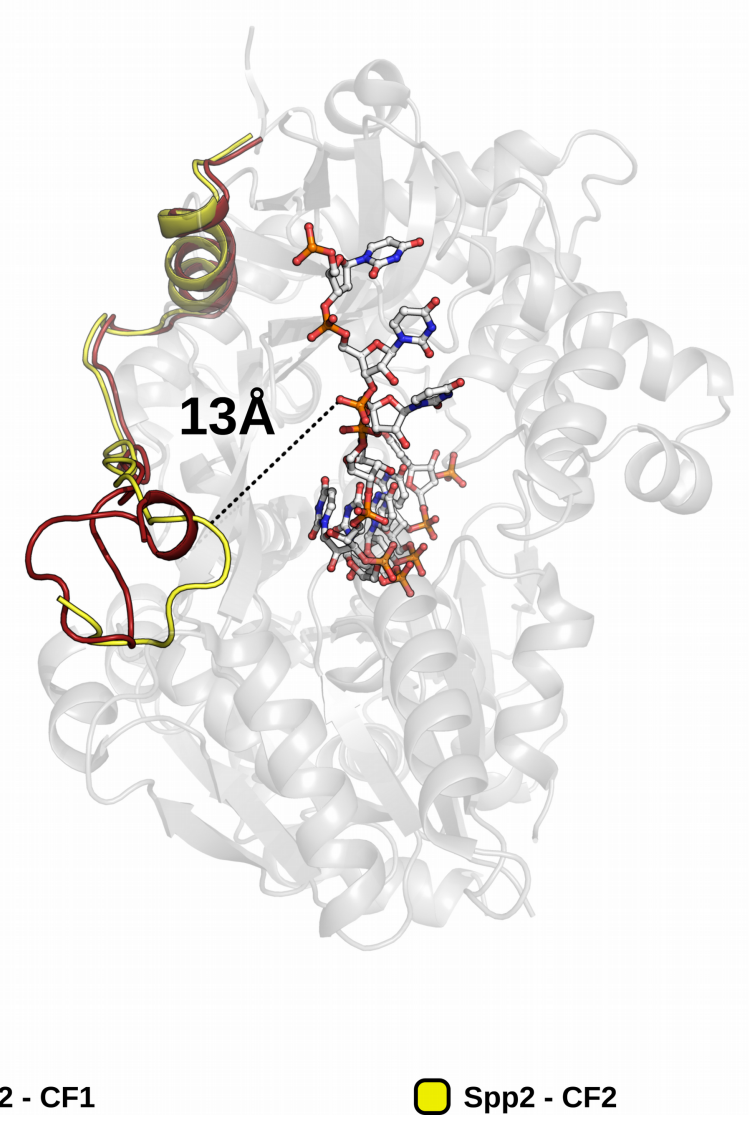

Fig. S11. Distance between the C-terminal end of the Spp2 G-patch and bound RNA

The position of the Spp2 G-patch in the ATP and Apo states was modeled by a superposition of the Prp2 RecA2 domain on the RecA2 domain of Prp43 (ATP) and Prp22 (Apo). In both states the closest distance of the G-patch to the RNA ranges between $12 \AA$ and $13 \AA$. This distance is too big to predict a direct interaction between the Spp2 G-patch and the RNA. 


\title{
Chapter 4: Structural analysis of the ADP-BeF${ }_{3}^{-}$- and RNA-bound DEAH-box ATPase Prp2
}

This manuscript is currently in preparation but requires additional experiments

\section{Structural analysis of the RNA- and ADP-BeF${ }_{3}^{-}$-bound DEAH-box ATPase Prp2}

\author{
Florian Hamann ${ }^{1}$, Ralf Ficner ${ }^{1 *}$
}

${ }^{1}$ Department of Molecular Structural Biology, Institute for Microbiology and Genetics, GZMB, GeorgAugust-University Göttingen, Justus-von-Liebig-Weg 11, 37077 Göttingen, Germany

*To whom correspondence should be addressed: rficner@gwdg.de

\section{Author contributions:}

F.H.: Experimental design, molecular cloning, protein expression and purification, crystallization, data collection and processing, structure determination, structure refinement and analysis, preparation of figures and tables, writing - original draft

R.F.: Experimental design, crystal structure analysis, data analysis, supervision, project leader 
Chapter 4: Structural analysis of the ADP-BeF3-- and RNA-bound DEAH-box ATPase Prp2

\section{Abstract}

Non-coding intron sequences present in precursor mRNAs need to be removed prior to translation and in higher eukaryotes they are excised via the spliceosome, a multi-megadalton molecular machine composed of numerous protein and RNA components. The DEAH-box ATPase Prp2 plays a crucial role during pre-mRNA splicing as it ensure the catalytic activation of the spliceosome. In contrast to other spliceosomal DEAH-box helicases, Prp2 seems not to function as an RNA-unwindase but rather as an RNA-dependent RNPase. Recent crystal structures of the spliceosomal DEAH-box ATPases Prp43 and Prp22 as well as of the closely related RHA helicase MLE in complex with RNA have contributed to a better understanding of how RNAbinding and processivity might be achieved in this helicase family. In order to shed light onto the divergent way of functioning of Prp2 we crystallized an N-terminally truncated construct of the Chaetomium thermophilum Prp2 in the presence of $\mathrm{ADP}-\mathrm{BeF}_{3}^{-}$and a poly- $\mathrm{U}_{12}$ RNA. The refined structure revealed a virtually identical conformation of the helicase core compared with the ADP-BeF${ }_{3}^{-}$- and RNA-bound structure of Prp43 and only a minor shift of the C-terminal domains. Interestingly, the stacked 3' RNA region shows an almost identical conformation in both structures, but at the 5' region the RNAs exhibit different conformations. While in Prp43 and Prp22 a kink in the RNA-backbone is introduced at the first position after the stack region, in Prp2 this nucleotide follows first the straight RNA-backbone path of the previous four nucleotides and a kink is introduced at a later position. We identified a loop in the C-terminal domains influencing the RNA conformation right after the 3' stack. In Prp43 a conserved serine and proline interact with the base at this position, whereby Prp2 exhibits an insertion in this loop inducing an alternative conformation of this loop, which is stabilized by an extensive interaction network with surrounding residues. We postulate that this different loop conformation alters the RNA-binding in a way that impedes Prp2 being an effective helicase.

\subsection{Introduction}

In eukaryotes most precursor messenger RNA (pre-mRNA) contain non-coding intron sequences which need to be removed in order to obtain a mature mRNA that can serve as a template for translation. The vast majority of these intervening sequences are removed with the help of the spliceosome (Matera and Wang, 2014; Wahl et al., 2009; Will and Lührmann, 2011). The spliceosome is a multimegadalton molecular machine that is sequentially assembled out of RNA and protein components. For each splicing cycle the complex is assemble de novo on a pre-mRNA and since it has no preformed active site, compositional as well as conformational rearrangements ensure the formation of a catalytically active complex. Once the 
Chapter 4: Structural analysis of the ADP-BeF3-- and RNA-bound DEAH-box ATPase Prp2

intron is excised via two subsequent transesterification steps, the complex is completely disassembled and each component is available for a new round of splicing.

These rearrangements need to be tightly orchestrated and are driven by a set of so-called DExD/H-box helicases that ensure the transition of the different spliceosomal complexes (Cordin et al., 2012; Ding and Pyle, 2012; Ozgur et al., 2015). The assembly steps are dominated by DEAD-box helicases, followed by the Ski2-like helicase Brr2 and all following activation, catalytic and disassembly steps are performed by DEAH-box ATPases. All of them belong to the helicase superfamily 2 (SF2) and have a helicase core composed of two RecA-like domains which harbor at least eight conserved sequence motifs (I, Ia, Ib, II, III, IV, V and VI) (Fairman-Williams et al., 2010). They play important roles in ATP binding and hydrolysis, RNA binding and coupling these processes to unwinding/translocation (Campodonico and Schwer, 2002; Schneider et al., 2004; Schwer and Meszaros, 2000). Additionally, Ski2-like and DEAH-box ATPases posses an auxiliary C-terminal domain that together with the helicase core form an RNA-binding tunnel (Büttner et al., 2007; Hamann et al., 2019; He et al., 2017; Prabu et al., 2015; Tauchert et al., 2017).

One key player during the catalytic activation of the spliceosome is the DEAH-box ATPase Prp2, which ensures the transition from the $\mathrm{B}^{\text {act }}$ to $\mathrm{B}^{*}$ complex (Kim and Lin, 1996; King and Beggs, 1990; Roy et al., 1995; Silverman et al., 2004). Here it is responsible for the destabilization of the SF3a/b complex, which exposes the 5' splice site and the branch-site enabling the first transesterification step (Bao et al., 2017; Lardelli et al., 2010; Ohrt et al., 2012; Warkocki et al., 2009). For its function it is strictly required to interact with the G-patch protein Spp2 (Krishnan et al., 2013; Roy et al., 1995; Silverman et al., 2004; Warkocki et al., 2015). Interestingly, Prp2 is the only spliceosomal DEAH-box ATPase that does not show any in vitro RNA unwinding activity (Christian et al., 2014; Schwer and Gross, 1998; Tanaka and Schwer, 2005; Tanaka et al., 2007; Tauchert et al., 2017; Wang et al., 1998). While recent biochemical evidence and cryo-EM structures suggest that DEAH-box ATPases likely act as translocases rather than unwindases in the spliceosome, Prp16, Prp22 and Prp43 still have the ability to unwind double-stranded RNA (Galej et al., 2016; Liu et al., 2017; Rauhut et al., 2016; Yan et al., 2016).

In order to investigate why Prp2 is not able to unwind RNA duplexes as the other closely related spliceosomal DEAH-box ATPases, we crystallized Prp2 in the presence of an ssRNA and the ATP-analog $\mathrm{ADP}_{-} \mathrm{BeF}_{3}$. Comparing this structure with ADP-bound Prp2 structures we were able to identify a yet undescribed connection between the nucleotide-binding site and the protein surface mediated by the movement of the conserved sequence motif III. This channel could serve as an exit passage for the hydrolyzed $Y$-phosphate or ensure exchange of water molecules with the active site. Additionally, we could describe an alternative mode of binding of the RNA in Prp2 with a kink in the RNA backbone which is shifted in position compared to RNA-bound Prp22 and Prp43 structures. We identified a loop in the C- 
Chapter 4: Structural analysis of the ADP-BeF3-- and RNA-bound DEAH-box ATPase Prp2

terminal domains to play an important role in threading the 5' RNA region, which strongly differs between Prp2 and Prp43. We postulate that this difference in RNA binding due to the influence of the C-terminal loop impedes Prp2 from being a competent unwindase.

\subsection{Material and methods}

\subsubsection{Protein production and crystallization}

Prp2 from Chaetomium thermophilum (ctPrp2) containing residues 286-921 was recombinantly expressed as a GST-tag fusion protein and purified as described in Schmitt et al. (2018). The complex containing $2 \mathrm{mg} \mathrm{ml}^{-}$ ${ }^{1}$ ctPrp2 $(27.4 \mu \mathrm{M})$, tenfold molar excess of ADP $(274 \mu \mathrm{M})$, twenty-fold molar excess of $\mathrm{BeSO}_{4}(548 \mu \mathrm{M})$, sixty-fold molar excess of $\mathrm{NaF}(1.644 \mathrm{mM})$ and 2.5-fold molar excess of poly- $\mathrm{U}_{12} \mathrm{RNA}(68.5 \mu \mathrm{M}$; Axolabs, Germany) was incubated for at least $30 \mathrm{~min}$ at $4{ }^{\circ} \mathrm{C}$ prior to the crystallization trials. The complex crystallized using the sitting-drop vapor-diffusion technique mixing $1 \mu \mathrm{l}$ complex solution with $1 \mu \mathrm{l}$ of the crystallization buffer. Crystals were grown in 100 mM MOPS/Na-HEPES pH 7.5, 8\% PEG20000, 22\% PEGMME 550 and 20 mM 1,6-hexanediol, 1-butanol, (RS)-1,2-propanediol, 2-propanol, 1,4-butandiol, 1,3-propanediol. Rodshaped crystals were obtained after 2 days of incubation at $20^{\circ} \mathrm{C}$.

\subsubsection{Data collection and processing}

Prior to data collection the crystals were cryoprotected with reservoir solution complemented with 5\% (w/v) PEG400 and 5\% (w/v) glycerol and flash-frozen in liquid nitrogen for storage. Oscillation images were collected at $100 \mathrm{~K}$ on beamline P14, PETRA III, DESY (Hamburg, Germany). Data processing was performed using the XDS package (Kabsch, 2010). X-ray diffraction data statistics are summarized in Table 1.

\subsubsection{Structure solution, refinement and analysis}

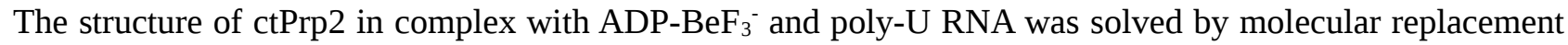
using Phaser (McCoy et al., 2007). The RecA1, RecA2 and C-terminal domains of the ADP-bound ctPrp2 structure (PDBid: 6fa5) were used as individual search models for molecular replacement (Schmitt et al., 2018). Due to a divergent conformation of Prp2 in this complex, no phasing solution could be found with an existing complete model. The model was manually built with Coot and refinements were performed with PHENIX, including TLS, weight optimization and bulk-solvent optimization (Adams et al., 2010). Validation 
tools in PHENIX and MolProbity were used to asses the final model quality (Chen et al., 2010). Superpositions of structures were performed with LSQMAN (Kleywegt, 1996) and figures were prepared with PyMOL (v.1.8, Schrödinger).

\subsection{Results}

\subsubsection{Overall conformation of $\mathrm{ADP}_{-} \mathrm{BeF}_{3}{ }^{-}-$and RNA-bound Prp2}

In order to investigate the discrepant way of functioning of Prp2 in terms of processivity, we crystallized Prp2 from Chaetomium thermophilum in presence of $\mathrm{ADP}_{-} \mathrm{BeF}_{3}{ }^{-}$and a $\mathrm{U}_{12}$-ssRNA, of which seven nucleotides were traceable in the electron density map. The used construct contains amino acids 286-921 and comprises the helicase core, composed of a RecA1 and RecA2 domain, and the C-terminal domains with its winged-helix (WH), helix-bundle (HB) and oligonucleotide-binding (OB) domain (Figure 1A). The truncated $\mathrm{N}$-terminal extension is only present with 10 amino acids, as it has been proven that the helicase core and the C-terminal domains are the key domains for the ATPase function of the DEAH-box family (Hamann et al., 2019; Tauchert et al., 2017).

Seven out of twelve RNA nucleotides were traceable in the electron density map. For $U_{2}$ only a model of the sugar-phosphate could be built. The ssRNA binds to an RNA-binding tunnel between the helicase core and the C-terminal domains, as already reported for the spliceosomal DEAH-box ATPases Prp43 and Prp22 (Figure 1A) (Hamann et al., 2019; He et al., 2017; Tauchert et al., 2017). The ssRNA interacts mainly with its sugar-phoshate backbone via polar interactions with Prp2 and leads to a sequence-unspecific binding. The only exception is $U_{3}$, as the base hydrogen bonds with Q516. RNA nucleotides $U_{4}-U_{7}$ are found in a stacked conformation and the backbone interacts with residues of the conserved sequence motifs (Ia: R352, R353; Ib: T395; IV: Q516; V: T572, N573) and conserved structural features (hook-turn: R380; hook-loop: S547; $\beta$ hairpin: K594). These interactions are identical among structurally characterized spliceosomal DEAH-box ATPases and ensure a stack of four RNA nucleotides in the ATP-bound state and a stack of five RNA nucleotides in the adenosine nucleotide-free state (Hamann et al., 2019; Tauchert et al., 2017). The $\mathrm{U}_{1}-\mathrm{U}_{3}$ stretch is only stabilized by three polar interactions, the base of $U_{3}$ with Q516 and the $U_{2}$ phosphate with H877 and T900 (Figure 1A).

The ATP-mimic ADP-BeF${ }_{3}^{-}$is sandwiched between both RecA-like domains (Figure 1A). Conserved residues comprising the active site bind the $\mathrm{ADP}_{-} \mathrm{BeF}_{3}{ }^{-}$in virtually identical manner as seen for the ctPrp43+ADP- $\mathrm{BeF}_{3}{ }^{-}$complex (Figure 1B) (Tauchert et al., 2017). This leads to an arrangement of the 
Chapter 4: Structural analysis of the ADP-BeF3-- and RNA-bound DEAH-box ATPase Prp2

helicase core that is conserved among DExH-box ATPases in the ATP-bound catalytic state (Figure 1C) (Chen et al., 2018a; Prabu et al., 2015; Tauchert et al., 2017).

\subsubsection{Putative exit channel for phosphate after ATP hydrolysis}

By analyzing differences in psi and phi angles of all available ctPrp2 structures, the regions exhibiting the greatest deviations in conformation were identified (Figure 2A). The flexibility of the $\beta$-hairpin and motif VI have already been discussed by Schmitt et al. (2018) and the role of the conformational variance of motif V was described by Hamann et al. (2019). Due to the rotation of the RecA2 domain between the ATP- and the ADP-bound state, the linker connecting both RecA-like domains also results in high differences of psi and phi angles. Interestingly, also motif III (SAT) exhibits increased conformational variability, which has not been described before. This motif has been proposed to play a role in coupling ATP hydrolysis to unwinding (Gross and Shuman, 1998; Heilek and Peterson, 1997; Pause and Sonenberg, 1992; Schwer and Meszaros, 2000). This motif is located close the phosphate moiety of the bound adenosine nucleotides and crystal structures of Prp2 in complex with $\mathrm{ADP}$ or $\mathrm{ADP}_{-} \mathrm{BeF}_{3}^{-}$show that it is able to adopt three different conformations (Figure 2C). Interestingly, in one of the ADP-bound structures (ctPrp2-ADP-CF1) it exhibits a conformation that forms a channel connecting the $\mathrm{Y}$-phosphate position of the active site with the surface of the protein. This channel could potentially display an exit passage for the resulting inorganic phosphate after ATP hydrolysis that has not been described yet. All other motif III conformations in the ADP-bound state do not allow the formation of a channel that connects the active site with the surface. In all ADP-bound crystal structures the H421 of the the eponymous DEAH-motif (motif II) interacts via a hydrogen bond with the mainchain nitrogen of A451, but only in ctPrp2-ADP-CF1 this alanine exhibits a conformation that ensure the start of an $\alpha$-helix at this position (Figure 2D). This alternative conformation displaces A451, enabling the formation of the channel. In the ATP-bound state this channel is as well occluded by motif III (Figure 2C). Here, H421 of motif II interacts with the sidechain of S450 and Q621 of motif VI hydrogen bonds with the sidechain of T452 (Figure 2E). Additionally, the A451 mainchain nitrogen, which in the ADP-bound state interacts with H421, is now involved in a hydrogen bond with the relay water of the active site (Dittrich and Schulten, 2005).

\subsubsection{Divergent 5' RNA conformations}

In all crystal structures of RNA-bound spliceosomal DEAH-box ATPases the 3' region of the ssRNA exhibits a stacked conformation that is stabilized by conserved interactions with the conserved sequence motifs of both RecA-like domains (Hamann et al., 2019; He et al., 2017; Tauchert et al., 2017). The stack 
accommodates either four or five RNA nucleotides depending on the adenosine nucleotide state and extends from the 3' end of the RNA up to the $\beta$-hairpin of the RecA2 domain. This structural feature ends the stack and redirects the 5' region of the RNA through the RNA-binding tunnel. While the stack of the 3' region seems to be conserved in terms of conformation, the 5' region shows different conformations in RNA-bound DExH-box ATPase crystal structures (Hamann et al., 2019). Interestingly, all spliceosomal DEAH-box ATPases exhibit a kink in the 5' region, but a superposition of the RecA2 domains reveals differences in the position of this kink in Prp2 (Figure 3). In the Prp2 crystal structure the kink is introduced significantly more distal when compared to the Prp43 or Prp22 structures, whereas it roughly overlaps when directly comparing the Prp43 and Prp22 structures (Figure 3). The analysis of the electrostatic potential of the DEAH-box ATPases shows that at the region of the RNA kink Prp2 is significantly more positively charged than Prp43 and Prp22 (Figure S1). This positively charged patch on Prp2 could contribute in guiding the RNA into an alternative 5' conformation with a shifted kink.

\subsubsection{Loop in C-terminal domain threads 5' RNA region through tunnel in the ATP-bound state}

The ctPrp43+ADP-BeF ${ }_{3}^{-}+\mathrm{RNA}$ structure is the only previously published genuine DEAH-box ATPase in the ATP- and RNA-bound state (Tauchert et al., 2017). A superposition highlights the virtually identical conformation of the helicase core and the highly similar position of the C-terminal domains (Figure 4A). The stacked 3' RNA region superposes as well to high degree, but at the first RNA nucleotide position after the stack the path of the RNAs differs. In the ctPrp43 structure a kink of the RNA backbone is introduced at this position mainly by the stacking of U3 with P557 and a hydrogen bond with S555 (Figure 4B). These two residues are part of a loop in the helix-bundle domain as part of the C-terminal domains. The respective loop in ctPrp2 displays an alternative conformation with a more extended $\alpha$-helix due to an insertion (Figure 4A). This conformation is stabilized by a network of polar interactions with surrounding residues (Figure 4C). T752 of the C-terminal loop hydrogen bonds with N548 from the hook-loop motif of the RecA2 domain, which itself interacts with R811 from the helix-bundle domain. R811 interacts as well with E749, which is stabilized by a hydrogen bond with S808. All these interactions lock the C-terminal loop in a conformation incompatible with an RNA kink as seen in the ctPrp43 structure. Instead, a kink in the RNA backbone is introduced at a position closer to the 5' end. While the conformation of the stacked 3' RNA region is highly conserved, the divergent conformations of the 5' RNA region seem to be highly influenced by the C-terminal loop. 


\subsubsection{Conservation of C-terminal loop}

The sequence alignment of the C-terminal loop reveals that it differs among the four spliceosomal DEAHbox ATPases but it is conserved for each individual ATPase (Figure 5A). While Prp43 and Prp16 share a highly conserved proline that stacks with a base of the RNA in the ctPrp43+ADP-BeF ${ }_{3}^{-}+\mathrm{RNA}$ structure, Prp22 has a conserved glutamine at this position. Prp2 is the only spliceosomal DEAH-box ATPase with an insertion in this loop, contributing to its unique conformation (Figure S2). Interestingly, although an insertion is conserved, the type of insertion differs among higher eukaryotes (Figure 5C). In the representatives of the fungi the glutamate and the threonine of the C-terminal loop are conserved. In some fungal members the threonine is replaced by a serine, which should still maintain the same interacting properties with the hookloop observed in the ctPrp2+ADP- $\mathrm{BeF}_{3}{ }^{-}+\mathrm{RNA}$ structure. In the representatives of the animals, the glutamate is not present and instead two asparagines are conserved.

The asparagine of the hook-loop interacting with the C-terminal loop in the ctPrp2+ADP-BeF${ }_{3}^{-}+\mathrm{RNA}^{2}$ structure is present in Prp2 of all analyzed organisms and suggests that the interplay between these two structural features is conserved in Prp2 (Figure 5B/D).

The conserved insertion of the C-terminal loop together with the interaction with the hook-loop leads to a unique conformation of the C-terminal loop in Prp2, which might play a regulatory role. In contrast, Cterminal loops of ctPrp43 and ctPrp22 show the same length of the $\alpha$-helix as well as a very similar conformation of the loop itself (Figure S2).

\subsection{Discussion}

Among the spliceosomal DEAH-box ATPases Prp2 plays a special role as up to date no dsRNA unwinding activity could be determined for this ATPase (Bao et al., 2017; Kim et al., 1992; Warkocki et al., 2015). In contrast, for Prp43, Prp22 and Prp16 in vitro helicase activity has been characterized (Christian et al., 2014; Schwer and Gross, 1998; Tanaka and Schwer, 2005; Tanaka et al., 2007; Tauchert et al., 2017; Wang et al., 1998). This raises the question why Prp2 functions so differently despite the sequence and structure similarity with the other spliceosomal DEAH-box ATPases. The elucidation of the determinant impeding Prp2 from being a competent unwindase might as well provide additional insights into regulatory aspects of

DEAH-box ATPases capable of disrupting duplex RNAs. In order to address these questions we solved the crystal structure of ctPrp2 with a bound $\mathrm{U}_{7}-\mathrm{RNA}$ and the ATP-analog $\mathrm{ADP}_{-} \mathrm{BeF}_{3}{ }^{-}$(Figure 1A).

Comparing the ATP-bound state of ctPrp2 with previously published ADP-bound structures we were able to identify significant conformational changes of the motif III between these states. This motif exhibits a closed conformation in the presence of $\mathrm{ADP}-\mathrm{BeF}_{3}{ }^{-}$, which is stabilized by polar contacts with neighboring motifs II 


\section{Chapter 4: Structural analysis of the ADP-BeF3-- and RNA-bound DEAH-box ATPase Prp2}

and VI (Figure 2E). It additionally interacts with the relay water of the adenosine nucleotide binding site. Active site water molecules are structurally highly conserved and specific to each nucleotide-bound state. In the ADP-bound state four water molecules are always coordinated by the active site magnesium, whereas in the ATP-bound state it coordinates three water molecules (He et al., 2010; Schmitt et al., 2018; Tauchert et al., 2016, 2017; Walbott et al., 2010). The ATP-bound state additionally harbors a catalytic and a relay water molecule present in all structures of this state (Dittrich and Schulten, 2005). The magnesium-coordinated water molecules as well as the catalytic water molecule have been proposed to play a crucial role in dictating the position of the RecA2 domain (Hamann et al., 2019). Due to the fact that these water molecules are specific for a certain catalytic state, a sensor serine in the RecA2 motif V is able to discriminate between the ADP- and the ATP-bound state in order to accordingly position the RecA2 domain. The relay water molecule might also function as an active site component that is sensed by motif III in order to induce the closed conformation of this motif. Conversely, this conformation might be needed to properly position the relay water for its role during ATP hydrolysis. Interestingly, one ADP-bound structure (PDBid: 6fac) exhibits an open conformation of motif III, which results in a direct connection of the nucleotide-binding site with the surface of the protein (Figure 2C). Up to date the exact order of events after the generation of ADP and $\mathrm{P}_{\mathrm{i}}$ is still unknown and it is not clear what is released first. For DEAD-box helicases it has been shown that the $\mathrm{P}_{\mathrm{i}}$ is released prior to the ADP (Wong et al., 2016). In the case that in the DEAH-box ATPase family the $P_{\mathrm{i}}$ is as well released first, another exit passage apart from the ATP entry/ADP exit site would be needed. The ctPrp2ADP-CF1 structure shows the first evidence for such a channel connecting the phosphate-bound end of the active site with the outer surface, thereby providing an alternative passage that would not be in conflict with the bound ADP. Alternatively, this channel could also function in the exchange of water molecules of the active site while the nucleotide remains bound. In order to determine the exact order of events during ATP hydrolysis in DEAH-box ATPases, detailed biochemical analyses as performed for the DEAD-box helicase Dbp5 are required (Wong et al., 2016).

Although recent structural and biochemical evidence suggest, that the spliceosomal DEAH-box ATPases might not need unwinding activity to fulfill their functions during splicing, Prp16, Prp22 and Prp43 show in vitro helicase activity (Christian et al., 2014; Schwer and Gross, 1998; Tanaka and Schwer, 2005; Tanaka et al., 2007; Tauchert et al., 2017; Wang et al., 1998). Prp2 is the only spliceosomal DEAH-box ATPase for which no in vitro unwinding activity has been determined (Bao et al., 2017; Kim et al., 1992). Since a divergent mode of interaction with RNA of Prp2 might lead to the lack of unwinding activity, we crystallized Prp2 in the presence of ssRNA and the ATP-analog $\mathrm{ADP}_{-} \mathrm{BeF}_{3}{ }^{-}$. A comparison with already published RNAbound structures of spliceosomal DEAH-box ATPases shows that, while all exhibit a kink of the RNA at the 5' region, it is shifted in the Prp2 structure compared to the ones from Prp43 and Prp22 (Figure 3). On the one hand, different electrostatic potentials of the ATPases close to this kink might influence the binding, and 
on the other hand a loop in the helix-bundle domain seems to play a crucial role in dictating the conformation of the RNA right after the interruption of the stack by the $\beta$-hairpin (Figure 4; Figure S1) (Hamann et al., 2019; Tauchert et al., 2017). Due to the strong dependence of the catalytic state on the overall conformation of DEAH-box ATPases, we primary compared the $\mathrm{ADP}_{-} \mathrm{BeF}_{3}{ }^{-}$- and RNA-bound structure of Prp2 with the structure of Prp43 in the same state. While the 3' stacked region of the RNA superimposes almost identically, the conformation of the 5' region shows major differences (Figure 4A). In the Prp43 structure the RNA is guided by an interaction with a proline and a serine of a loop protruding out of the C-terminal domains (Figure 4A/B). This interaction ensures the kink in the RNA directly after the $\beta$-hairpin. Prp2 lacks such an interaction with the RNA at this position and instead this loop harbors a conserved insertion that ensures an alternative conformation of this loop, which is stabilized by multiple interactions with neighboring residues (Figure 4C). One of these interactions is with N548, which is part of the hook-loop (Prabu et al., 2015). Interestingly, this structural feature has been proposed to play an important role in processivity in the DExHbox helicase MLE. However, it has also been shown that the hook-loop is dispensable for helicase function in Prp43 (Tauchert et al., 2017). The hook-loop and the C-terminal loop are in close proximity and might play similar roles in threading the RNA through the RNA-binding tunnel in the 5' region. Since the RNA in Prp43 primarily interacts with the C-terminal loop in the 5' region, the interaction with the hook-loop might be circumvented. The hook-loop as well as the C-terminal loop show only low sequence conservation among the four spliceosomal DEAH-box ATPases, but are conserved for each DEAH-box ATPase (Figure 5A/B). This suggests that the different spliceosomal DEAH-box ATPases have a different conserved ways of threading the RNA through the tunnel after the stacked 3' region. Prp43 utilizes mainly a conserved proline and serine of the C-terminal loop and exhibits short residues in the hook-loop that do not intervene in the binding. Although Prp16 has a highly similar C-terminal loop, the hook-loop has a different property and might induce a different RNA conformation as compared with Prp43. Prp22 has a unique glutamine instead of a proline in the C-terminal loop and the sequence of the hook-loop is also different to the other ones. Unfortunately, only an RNA-bound but nucleotide-free structure of Prp22 is available, where the RecA2 domain is differently positioned and thereby the C-terminal loop and the hook-loop are spatially apart (Hamann et al., 2019). In this structure these two structural features do not interact with each other and the C-terminal loop does not contact the RNA. The overall conformation of the C-terminal loop in Prp22 closely

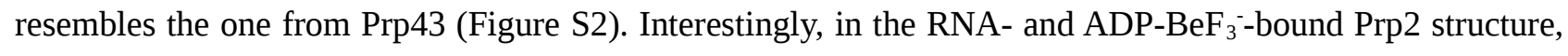
the C-terminal loop displays a distinct conformation due to an insertion. Instead of interacting with the RNA, it interacts with a conserved asparagine of the hook-loop and other surrounding residues (Figure 4C). This interaction network is likely conserved and threads the 5' RNA region in a completely different manner compared to Prp43 and Prp22 (Figure 3). Interestingly, the conservation of the C-terminal loop shows major differences in sequence between representatives of the fungi and animalia, but in both cases an insertion 
Chapter 4: Structural analysis of the ADP-BeF3-- and RNA-bound DEAH-box ATPase Prp2

seems to be conserved as a feature of this structural motif in Prp2 (Figure 5C). Despite this difference in sequence of the C-terminal loop, the hook-loop is consistently conserved among fungi and animalia, suggesting a similar conformation and interaction network for the animalia as seen for the Chaetomium thermophilum Prp2 as a candidate of the fungi (Figure 5D). We postulate that this difference in interaction mode with the RNA impedes Prp2 from being able to perform in vitro unwinding of duplex RNA. We further on suggest that in some DEAH-box ATPases there might by an interplay between the C-terminal loop and the hook-loop that dictates how the 5' RNA region is threaded and thereby play a regulatory role. The proper positioning of the 5' region might not only be relevant for unwinding capabilities, but specially for the spliceosomal DEAH-box ATPases it might be crucial for correctly interacting with the ssRNA targets in order to translocate on them. 
Chapter 4: Structural analysis of the ADP-BeF3-- and RNA-bound DEAH-box ATPase Prp2

\section{References}

1. Will,C.L. and Lührmann,R. (2011) Spliceosome structure and function. Cold Spring Harb Perspect Biol, 3.

2. Wahl,M.C., Will,C.L. and Lührmann,R. (2009) The spliceosome: design principles of a dynamic RNP machine. Cell, 136, 701-718.

3. Matera,A.G. and Wang,Z. (2014) A day in the life of the spliceosome. Nat. Rev. Mol. Cell Biol., 15, 108121.

4. Cordin,O., Hahn,D. and Beggs,J.D. (2012) Structure, function and regulation of spliceosomal RNA helicases. Curr. Opin. Cell Biol., 24, 431-438.

5. Ding,S.C. and Pyle,A.M. (2012) Molecular mechanics of RNA translocases. Meth. Enzymol., 511, 131147.

6. Ozgur,S., Buchwald,G., Falk,S., Chakrabarti,S., Prabu,J.R. and Conti,E. (2015) The conformational plasticity of eukaryotic RNA-dependent ATPases. FEBS J., 282, 850-863.

7. Fairman-Williams,M.E., Guenther,U.-P. and Jankowsky,E. (2010) SF1 and SF2 helicases: family matters. Curr. Opin. Struct. Biol., 20, 313-324.

8. Schwer,B. and Meszaros,T. (2000) RNA helicase dynamics in pre-mRNA splicing. EMBO J., 19, 65826591.

9. Campodonico,E. and Schwer,B. (2002) ATP-Dependent Remodeling of the Spliceosome: Intragenic Suppressors of Release-Defective Mutants of Saccharomyces cerevisiae Prp22. Genetics, 160, 407-415.

10. Schneider,S., Campodonico,E. and Schwer,B. (2004) Motifs IV and V in the DEAH box splicing factor Prp22 are important for RNA unwinding, and helicase-defective Prp22 mutants are suppressed by Prp8. J. Biol. Chem., 279, 8617-8626.

11. Büttner,K., Nehring,S. and Hopfner,K.-P. (2007) Structural basis for DNA duplex separation by a superfamily-2 helicase. Nature Structural \& Molecular Biology, 14, 647-652.

12. He,Y., Staley,J.P., Andersen,G.R. and Nielsen,K.H. (2017) Structure of the DEAH/RHA ATPase Prp43p bound to RNA implicates a pair of hairpins and motif Va in translocation along RNA. RNA, 23, 1110-1124.

13. Prabu,J.R., Müller,M., Thomae,A.W., Schüssler,S., Bonneau,F., Becker,P.B. and Conti,E. (2015) Structure of the RNA Helicase MLE Reveals the Molecular Mechanisms for Uridine Specificity and RNAATP Coupling. Molecular Cell, 60, 487-499.

14. Tauchert,M.J., Fourmann,J.-B., Lührmann,R. and Ficner,R. (2017) Structural insights into the mechanism of the DEAH-box RNA helicase Prp43. eLife, 6, e21510.

15. Hamann,F., Enders,M. and Ficner,R. (2019) Structural basis for RNA translocation by DEAH-box ATPases. Nucleic Acids Res., 10.1093/nar/gkz150. 
Chapter 4: Structural analysis of the ADP-BeF3-- and RNA-bound DEAH-box ATPase Prp2

16. King,D.S. and Beggs,J.D. (1990) Interactions of PRP2 protein with pre-mRNA splicing complexes in Saccharomyces cerevisiae. Nucleic Acids Res., 18, 6559-6564.

17. Roy,J., Kim,K., Maddock,J.R., Anthony,J.G. and Woolford,J.L. (1995) The final stages of spliceosome maturation require Spp2p that can interact with the DEAH box protein Prp2p and promote step 1 of splicing. RNA, 1, 375-390.

18. Kim,S.H. and Lin,R.J. (1996) Spliceosome activation by PRP2 ATPase prior to the first transesterification reaction of pre-mRNA splicing. Mol. Cell. Biol., 16, 6810-6819.

19. Silverman,E.J., Maeda,A., Wei,J., Smith,P., Beggs,J.D. and Lin,R.-J. (2004) Interaction between a Gpatch protein and a spliceosomal DEXD/H-box ATPase that is critical for splicing. Mol. Cell. Biol., 24, 10101-10110.

20. Ohrt,T., Prior,M., Dannenberg,J., Odenwälder,P., Dybkov,O., Rasche,N., Schmitzová,J., Gregor,I., Fabrizio,P., Enderlein,J., et al. (2012) Prp2-mediated protein rearrangements at the catalytic core of the spliceosome as revealed by dcFCCS. RNA, 18, 1244-1256.

21. Lardelli,R.M., Thompson,J.X., Yates,J.R. and Stevens,S.W. (2010) Release of SF3 from the intron branchpoint activates the first step of pre-mRNA splicing. RNA, 16, 516-528.

22. Warkocki,Z., Odenwälder,P., Schmitzová,J., Platzmann,F., Stark,H., Urlaub,H., Ficner,R., Fabrizio,P. and Lührmann,R. (2009) Reconstitution of both steps of Saccharomyces cerevisiae splicing with purified spliceosomal components. Nat. Struct. Mol. Biol., 16, 1237-1243.

23. Bao,P., Höbartner,C., Hartmuth,K. and Lührmann,R. (2017) Yeast Prp2 liberates the 5' splice site and the branch site adenosine for catalysis of pre-mRNA splicing. RNA, 23, 1770-1779.

24. Warkocki,Z., Schneider,C., Mozaffari-Jovin,S., Schmitzová,J., Höbartner,C., Fabrizio,P. and Lührmann,R. (2015) The G-patch protein Spp2 couples the spliceosome-stimulated ATPase activity of the DEAH-box protein Prp2 to catalytic activation of the spliceosome. Genes Dev., 29, 94-107.

25. Krishnan,R., Blanco,M.R., Kahlscheuer,M.L., Abelson,J., Guthrie,C. and Walter,N.G. (2013) Biased Brownian ratcheting leads to pre-mRNA remodeling and capture prior to first-step splicing. Nature Structural \& Molecular Biology, 20, 1450-1457.

26. Tanaka,N., Aronova,A. and Schwer,B. (2007) Ntr1 activates the Prp43 helicase to trigger release of lariat-intron from the spliceosome. Genes Dev., 21, 2312-2325.

27. Christian,H., Hofele,R.V., Urlaub,H. and Ficner,R. (2014) Insights into the activation of the helicase Prp43 by biochemical studies and structural mass spectrometry. Nucleic Acids Res., 42, 1162-1179.

28. Schwer,B. and Gross,C.H. (1998) Prp22, a DExH-box RNA helicase, plays two distinct roles in yeast pre-mRNA splicing. EMBO J, 17, 2086-2094.

29. Tanaka,N. and Schwer,B. (2005) Characterization of the NTPase, RNA-Binding, and RNA Helicase Activities of the DEAH-Box Splicing Factor Prp22. Biochemistry, 44, 9795-9803.

30. Wang,Y., Wagner,J.D. and Guthrie,C. (1998) The DEAH-box splicing factor Prp16 unwinds RNA duplexes in vitro. Curr. Biol., 8, 441-451. 
Chapter 4: Structural analysis of the ADP-BeF3-- and RNA-bound DEAH-box ATPase Prp2

31. Liu,S., Li,X., Zhang,L., Jiang,J., Hill,R.C., Cui,Y., Hansen,K.C., Zhou,Z.H. and Zhao,R. (2017) Structure of the yeast spliceosomal postcatalytic P complex. Science, 358, 1278-1283.

32. Rauhut,R., Fabrizio,P., Dybkov,O., Hartmuth,K., Pena,V., Chari,A., Kumar,V., Lee,C.-T., Urlaub,H., Kastner,B., et al. (2016) Molecular architecture of the Saccharomyces cerevisiae activated spliceosome. Science, 353, 1399-1405.

33. Galej,W.P., Wilkinson,M.E., Fica,S.M., Oubridge,C., Newman,A.J. and Nagai,K. (2016) Cryo-EM structure of the spliceosome immediately after branching. Nature, 537, 197-201.

34. Yan,C., Wan,R., Bai,R., Huang,G. and Shi,Y. (2016) Structure of a yeast activated spliceosome at $3.5 \AA$ resolution. Science, 353, 904-911.

35. Kabsch,W. (2010) XDS. Acta Crystallogr. D Biol. Crystallogr., 66, 125-132.

36. McCoy,A.J., Grosse-Kunstleve,R.W., Adams,P.D., Winn,M.D., Storoni,L.C. and Read,R.J. (2007) Phaser crystallographic software. J Appl Crystallogr, 40, 658-674.

37. Schmitt,A., Hamann,F., Neumann,P. and Ficner,R. (2018) Crystal structure of the spliceosomal DEAHbox ATPase Prp2. Acta Crystallogr D Struct Biol, 74, 643-654.

38. Adams,P.D., Afonine,P.V., Bunkoczi,G., Chen,V.B., Davis,I.W., Echols,N., Headd,J.J., Hung,L.-W., Kapral,G.J., Grosse-Kunstleve,R.W., et al. (2010) PHENIX: a comprehensive Python-based system for macromolecular structure solution. Acta Crystallogr. Sect. D-Biol. Crystallogr., 66, 213-221.

39. Chen,V.B., Arendall,W.B., Headd,J.J., Keedy,D.A., Immormino,R.M., Kapral,G.J., Murray,L.W., Richardson,J.S. and Richardson,D.C. (2010) MolProbity: all-atom structure validation for macromolecular crystallography. Acta Crystallogr. D Biol. Crystallogr., 66, 12-21.

40. Kleywegt,G.J. (1996) Use of Non-crystallographic Symmetry in Protein Structure Refinement. Acta Cryst D, 52, 842-857.

41. Chen,M.C., Tippana,R., Demeshkina,N.A., Murat,P., Balasubramanian,S., Myong,S. and FerréD’Amaré,A.R. (2018) Structural basis of G-quadruplex unfolding by the DEAH/RHA helicase DHX36. Nature, 558, 465.

42. Gross,C.H. and Shuman,S. (1998) The nucleoside triphosphatase and helicase activities of vaccinia virus NPH-II are essential for virus replication. J. Virol., 72, 4729-4736.

43. Heilek,G.M. and Peterson,M.G. (1997) A point mutation abolishes the helicase but not the nucleoside triphosphatase activity of hepatitis C virus NS3 protein. J. Virol., 71, 6264-6266.

44. Pause,A. and Sonenberg,N. (1992) Mutational analysis of a DEAD box RNA helicase: the mammalian translation initiation factor eIF-4A. EMBO J., 11, 2643-2654.

45. Dittrich,M. and Schulten,K. (2005) Zooming in on ATP hydrolysis in F1. J. Bioenerg. Biomembr., 37, 441-444.

46. Kim,S.H., Smith,J., Claude,A. and Lin,R.J. (1992) The purified yeast pre-mRNA splicing factor PRP2 is an RNA-dependent NTPase. EMBO J., 11, 2319-2326. 
Chapter 4: Structural analysis of the ADP-BeF3-- and RNA-bound DEAH-box ATPase Prp2

47. Tauchert,M.J., Fourmann,J.-B., Christian,H., Lührmann,R. and Ficner,R. (2016) Structural and functional analysis of the RNA helicase Prp43 from the thermophilic eukaryote Chaetomium thermophilum. Acta Crystallogr F Struct Biol Commun, 72, 112-120.

48. Walbott,H., Mouffok,S., Capeyrou,R., Lebaron,S., Humbert,O., van Tilbeurgh,H., Henry,Y. and Leulliot,N. (2010) Prp43p contains a processive helicase structural architecture with a specific regulatory domain. EMBO J., 29, 2194-2204.

49. He,Y., Andersen,G.R. and Nielsen,K.H. (2010) Structural basis for the function of DEAH helicases. EMBO Rep, 11, 180-186.

50. Wong,E.V., Cao,W., Vörös,J., Merchant,M., Modis,Y., Hackney,D.D., Montpetit,B. and De La Cruz,E.M. (2016) P(I) Release Limits the Intrinsic and RNA-Stimulated ATPase Cycles of DEAD-Box Protein 5 (Dbp5). J. Mol. Biol., 428, 492-508. 
Chapter 4: Structural analysis of the ADP-BeF3-- and RNA-bound DEAH-box ATPase Prp2

Table 1: X-ray and refinement statistics

\begin{tabular}{|c|c|}
\hline Data collection & Prp2 + $\mathrm{ADP}-\mathrm{BeF}_{3}{ }^{-}+\mathrm{RNA}$ \\
\hline $\begin{array}{l}\text { Space group } \\
\text { a, b, c }(\AA)\end{array}$ & $\begin{array}{l}\mathrm{P} 2{ }_{1}{ }_{1}{ }_{2} 1 \\
48.66,100.41,140.95\end{array}$ \\
\hline$\alpha, \beta, \gamma\left(^{\circ}\right)$ & $90,90,90$ \\
\hline X-ray source & P14, PETRA III, DESY \\
\hline Resolution range $(\AA)$ & $50.0-2.1(2.2-2.1)$ \\
\hline $\begin{array}{l}\text { No. of unique } \\
\text { reflections }\end{array}$ & $41019(5228)$ \\
\hline Completeness (\%) & $99.5(99.0)$ \\
\hline$R_{\text {merge }}(\%)$ & $12.6(127.9)$ \\
\hline Average $\mathrm{I} / \sigma(\mathrm{I})$ & $14.88(2.21)$ \\
\hline Redundancy & $13.68(13.90)$ \\
\hline $\mathrm{CC}_{1 / 2}$ & $99.9(81.3)$ \\
\hline Wilson $B\left(\AA^{2}\right)$ & 42.3 \\
\hline \multicolumn{2}{|l|}{ Refinement } \\
\hline Resolution $(\AA)$ & $81.78-2.10(2.15-2.10)$ \\
\hline No. of reflections & 41000 \\
\hline $\mathrm{R}_{\text {work }}(\%)$ & $21.11(27.51)$ \\
\hline $\mathrm{R}_{\text {free }}(\%)$ & $23.66(29.78)$ \\
\hline $\begin{array}{l}\text { Molecules per } \\
\text { asymmetric unit }\end{array}$ & 1 \\
\hline Total number of atoms & 5068 \\
\hline Protein residues & 625 \\
\hline Water molecules & 99 \\
\hline \multicolumn{2}{|l|}{ r.m.s. deviations } \\
\hline Bond length $(\AA)$ & 0.002 \\
\hline Bond angles $\left({ }^{\circ}\right)$ & 0.591 \\
\hline \multicolumn{2}{|l|}{$\begin{array}{l}\text { Ramachandran } \\
\text { statistics }\end{array}$} \\
\hline favoured (\%) & 96.94 \\
\hline allowed (\%) & 2.90 \\
\hline outliers (\%) & 0.16 \\
\hline
\end{tabular}


A

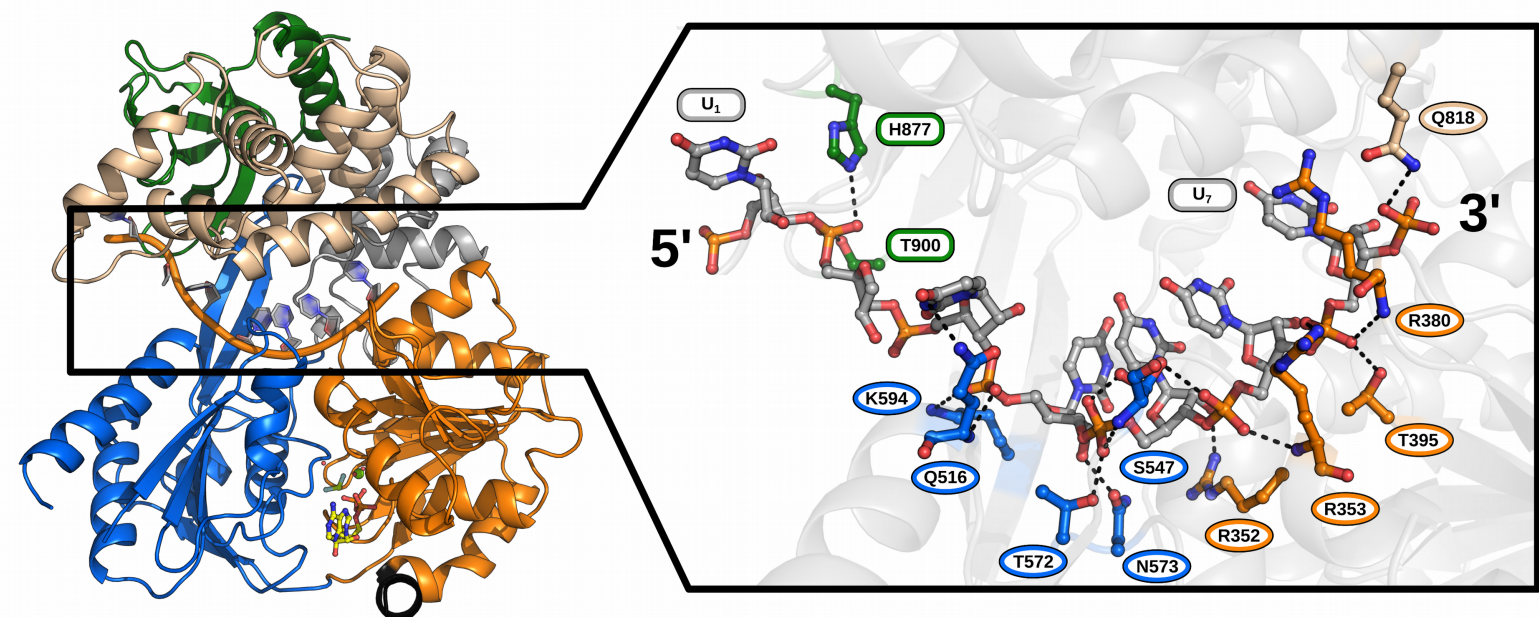

\begin{tabular}{|l|l|l|l|l|}
\hline RecA1 & RecA2 & WH & HB & OB \\
\hline
\end{tabular}

B

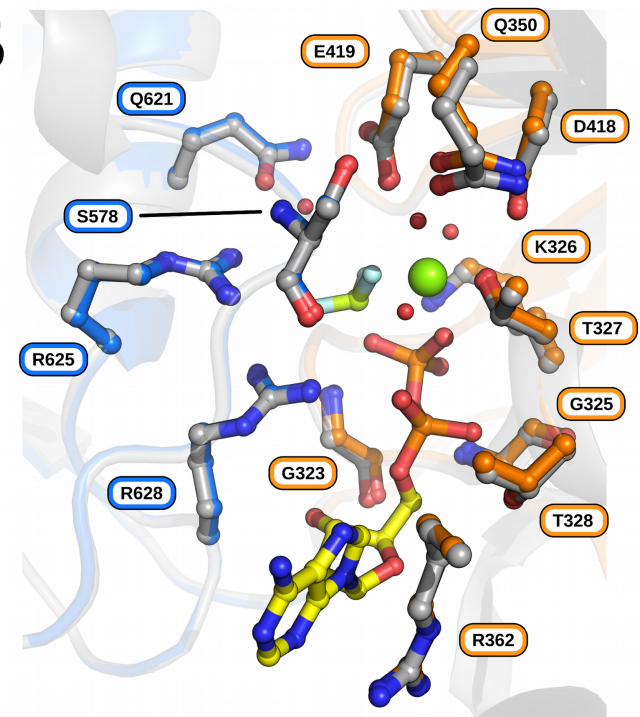

D ctPrp2+ADP-BeF ${ }^{-}+\mathrm{RNA}^{-}$ (PDBid: $X X X X)$
ctPrp43+ADP-BeF ${ }_{3}^{-}$ (PDBid: 5ltj)

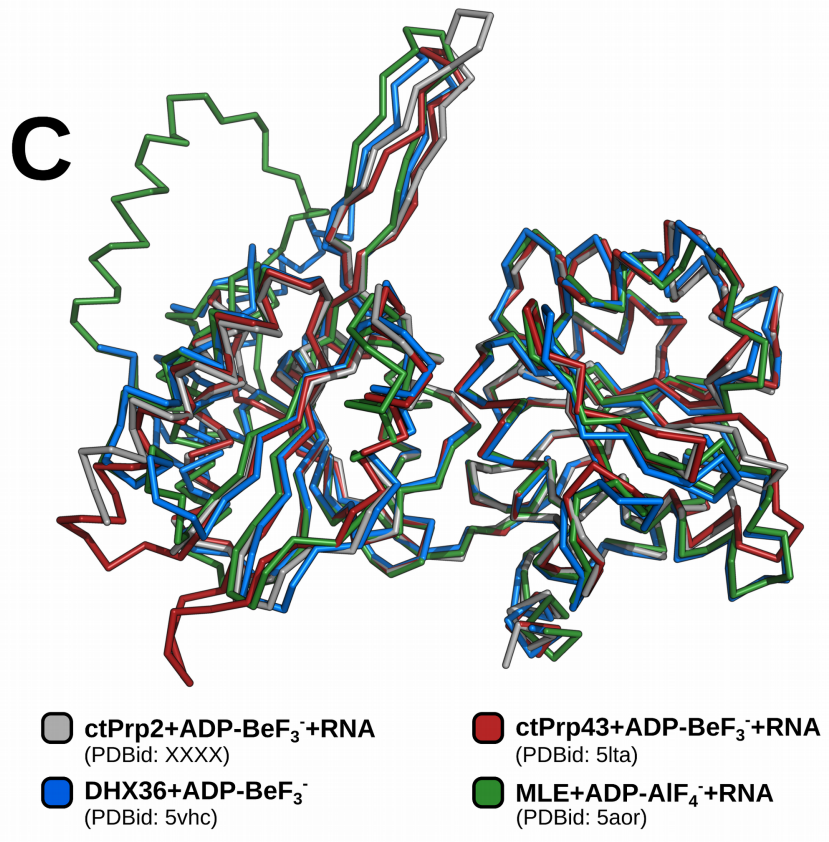

(PDBid: 5vhc)

Figure 1: Structural overview of the pre-catalytic state of Prp2. (A) Prp2 and the bound RNA are displayed as a cartoon model and the $\mathrm{ADP}_{-} \mathrm{BeF}_{3}{ }^{-}$is depicted as sticks. The crystallized construct is composed of two RecA-like domains (RecA1: orange; RecA2: blue), a winged-helix (WH: gray), a helix-bundle (HB: wheat) and a oligonucleotidebinding (OB: green) domain. The RNA is bound between the helicase core and the C-terminal domains and the nucleotide is sandwiched between both RecA-like domains. Conserved residues interacting with the RNA backbone of the 3' stacked region are shown in circles, whereby remaining interacting residues are shown in a rectangular shape. (B) Superposition of active site residues of Prp2 and Prp43 interacting with the ADP-BeF ${ }_{3}^{-}$. Both DEAH-box ATPases interact in the identical manner with the nucleotide. (C) Superposition of all structurally characterized DExH-box ATPases bound to an ATP-analog. The conformation of the helicase core in the ATP-bound state is highly conserved. 


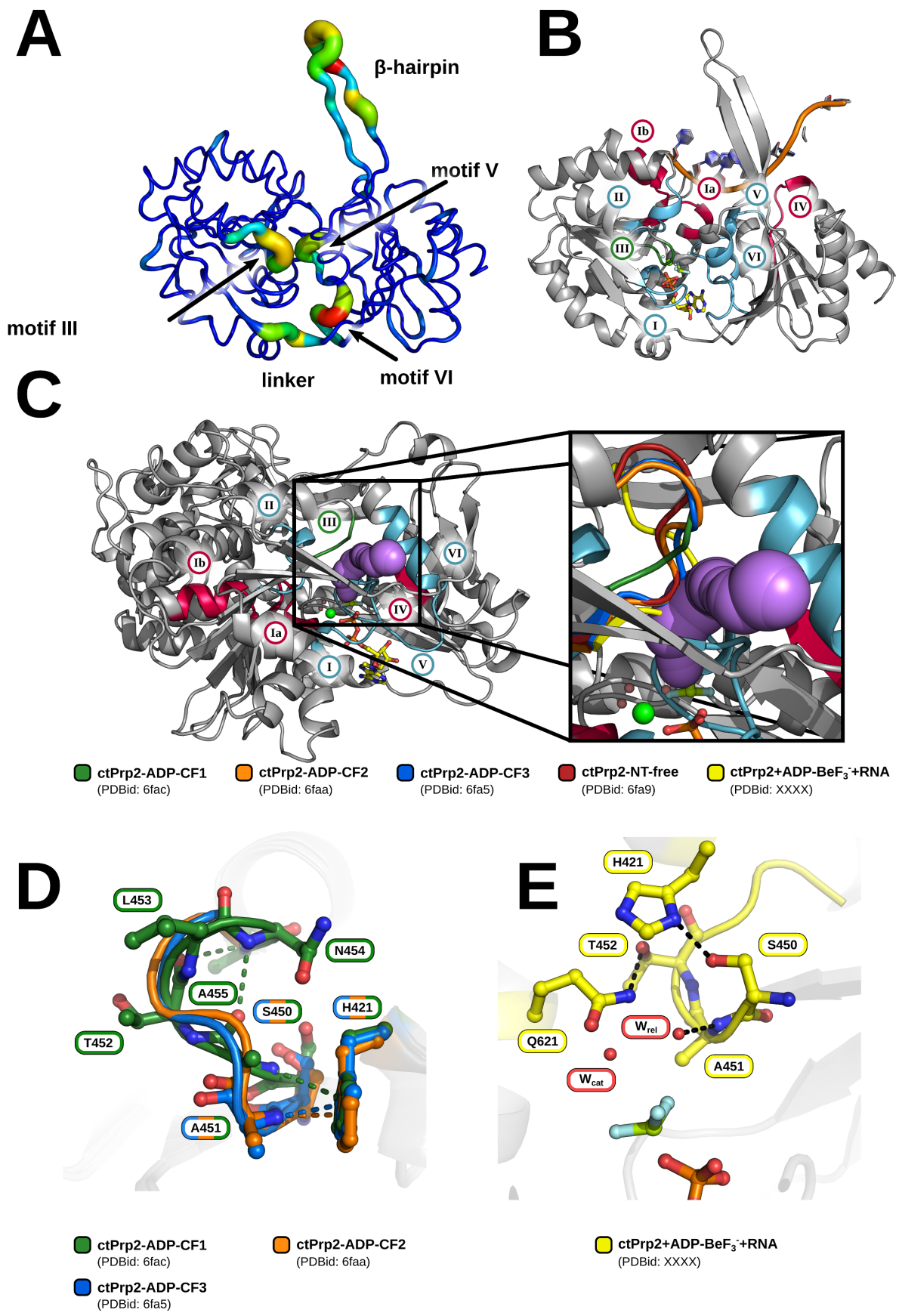

Figure 2: Movements of conserved sequence motif III control the formation of a channel connecting the nucleotide-binding site with the protein surface. (A) Differences of psi and phi angles for single residues of the helicase core of all available Prp2 structures are plotted onto the Prp2 structure. Motifs III, V and VI as well as the $\beta$ hairpin and the linker connecting both RecA-like domains show movements of the mainchain. (B) Overview of conserved sequence motifs spread over the two RecA-like domains. (C) In the ctPrp2-ADP-CF1 structure, motif III adopts a conformation that allows the formation of a channel that connects the $\gamma$-phosphate position of the active site with the exterior of the protein. In other ADP- and $\mathrm{ADP}-\mathrm{BeF}_{3}{ }^{-}$-bound structures motif III closes this channel. (D) Overview of motif III interactions in the ADP-bound state. (E) Overview of motif III interactions in the ADP-BeF ${ }_{3}^{-}$bound state. 

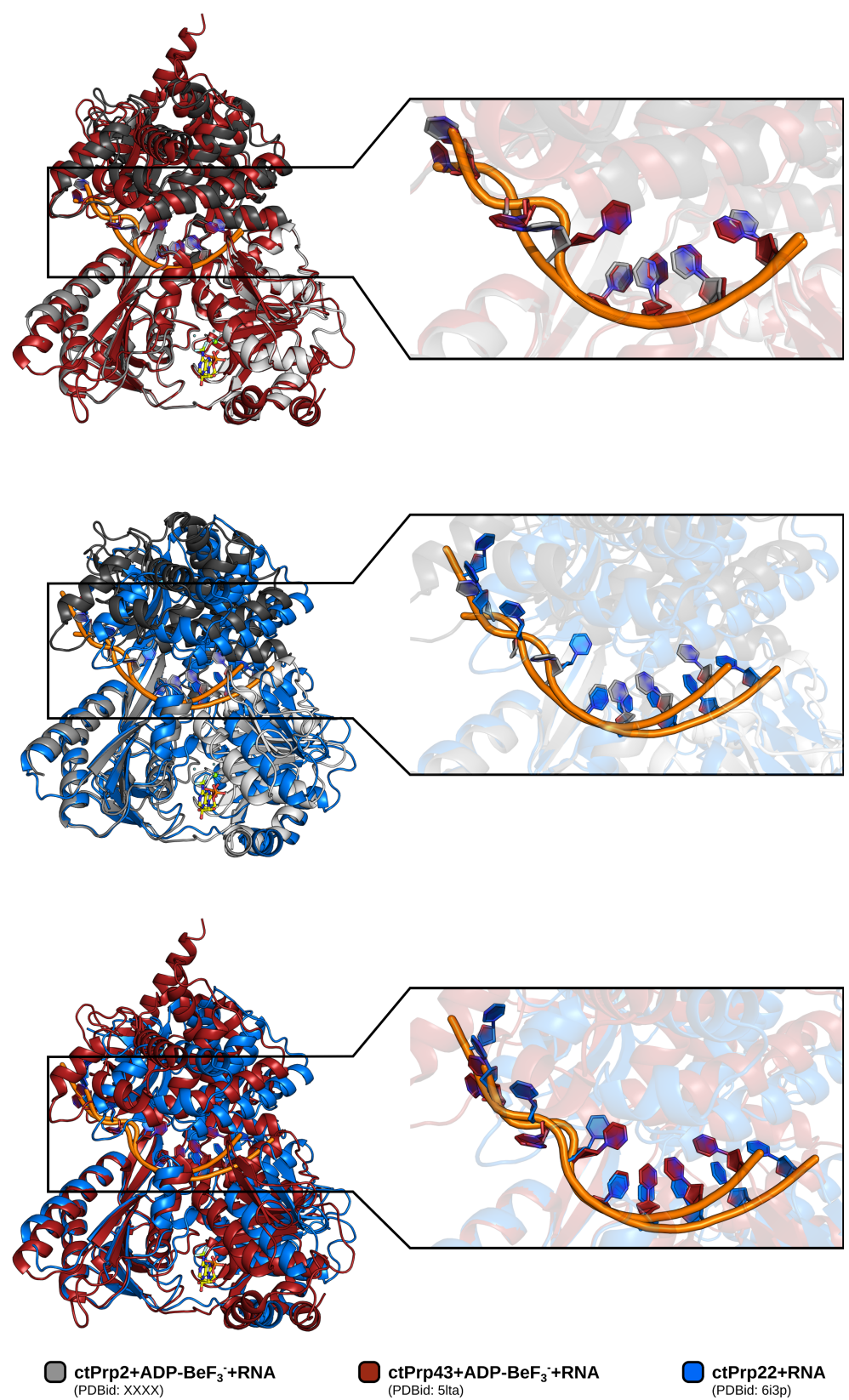

Figure 3: Comparison of ssRNA binding to spliceosomal DEAH-box ATPases. All ssRNAs bound to spliceosomal DEAH-box ATPases exhibit a kink in the 5' region. When the RecA2 domains are superimposed, the kink in the Prp43 and Prp22 structures share a similar position and only the kink in the Prp2 structure is differently positioned. 
A

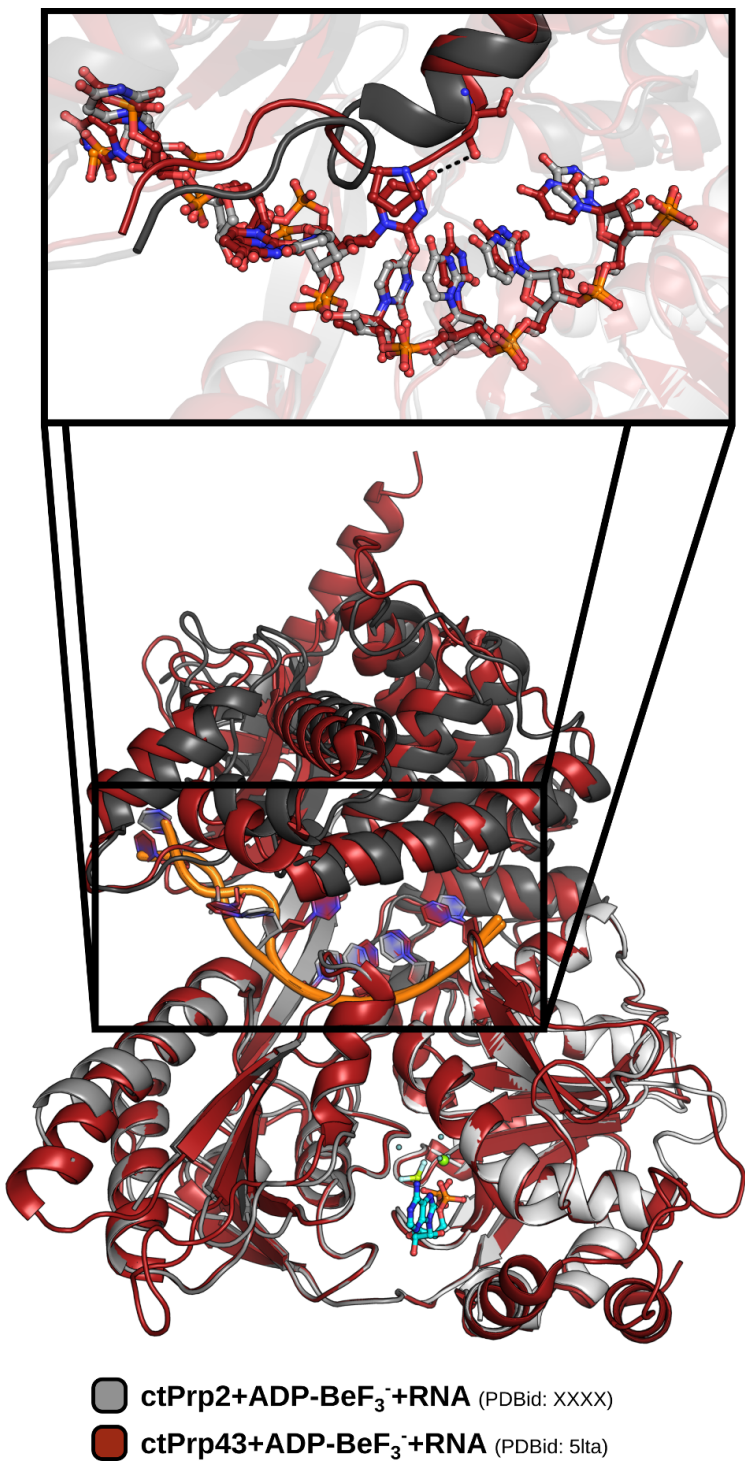

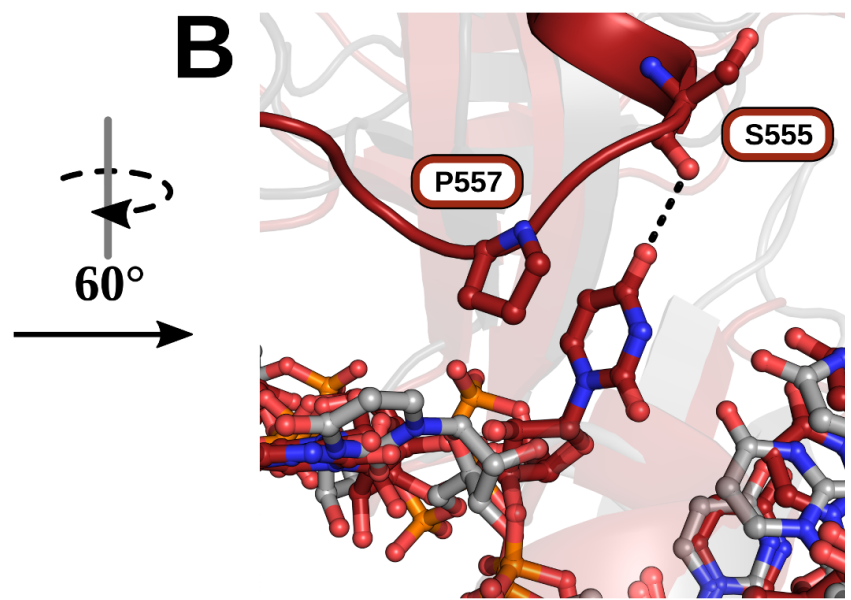
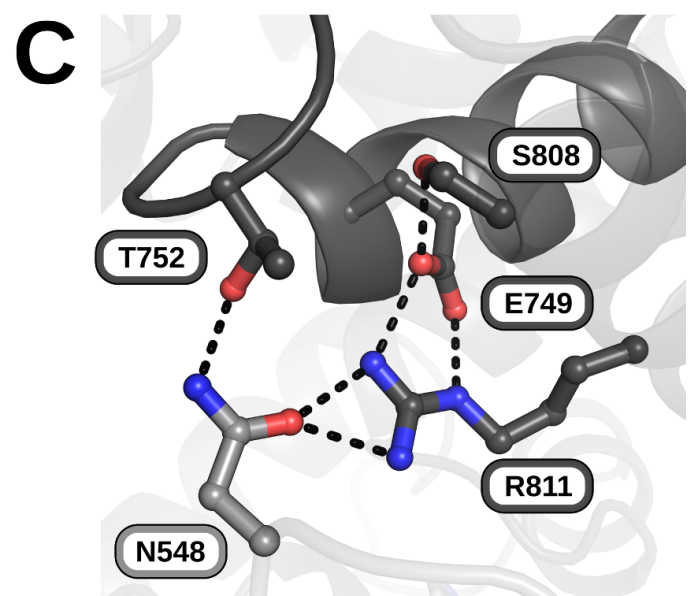

Figure 4: C-terminal loop dictates conformation of 5' RNA region. (A) Superposition of Prp2 and Prp43 in the precatalytic state via the helicase core. The 3' stacked RNA region superposes almost identically, but at the beginning of the 5' region, the RNA bound to Prp43 interacts with the C-terminal loop. This loop has a different conformation in Prp2 and does not interact with the RNA. (B) The base of the first nucleotide of the 5' RNA region interacts with a proline and serine of the Prp43 C-terminal loop. (C) The alternative conformation of the Prp2 C-terminal loop is stabilized by interactions with surrounding residues belonging to the helix-bundle domain and the hook-loop of the RecA2 domain. 


\section{C-terminal Hook-loop loop}

\begin{tabular}{llll}
\hline ctPrp2 & 747 & LGEVGT \\
scPrp2 & 678 & LHETPS \\
hsPrp2 & 847 & LSVNNS \\
xlPrp2 & 838 & LSVNNT \\
cePrp2 & 813 & LSCNAA \\
ctPrp43 & 554 & LSVP.Q \\
scPrp43 & 549 & LSVP.N \\
hsPrp43 & 594 & LSVP.Q \\
xlPrp43 & 560 & LSVP.Q \\
cePrp43 & 538 & LSVP.Q \\
ctPrp16 & 754 & LSVP.N \\
scPrp16 & 812 & LSVP.Q \\
hsPrp16 & 978 & LSVP.A \\
xlPrp16 & 982 & LSVP.A \\
cePrp16 & 888 & LSVP.A \\
ctPrp22 & 1004 & LNLQ. Q \\
scPrp22 & 930 & LSVQ.N \\
hsPrp22 & 1012 & LSVQ.N \\
xtPrp22 & 1006 & LSVQ.N \\
cePrp22 & 987 & LNVQ.N
\end{tabular}

\begin{tabular}{llll}
\hline ctPrp2 & 546 & $\mathrm{YSN}$ \\
scPrp2 & 475 & $\mathrm{YAN}$ \\
hsPrp2 & 648 & $\mathrm{YAN}$ \\
xIPrp2 & 639 & $\mathrm{YAN}$ \\
cePrp2 & 614 & $\mathrm{YAN}$ \\
ctPrp43 & 348 & $\mathrm{YGT}$ \\
scPrp43 & 345 & $\mathrm{YGS}$ \\
hsPrp43 & 389 & $\mathrm{YST}$ \\
xIPrp43 & 356 & $\mathrm{YST}$ \\
cePrp43 & 333 & $\mathrm{YST}$ \\
ctPrp16 & 555 & $\mathrm{YSQ}$ \\
scPrp16 & 612 & $\mathrm{YSA}$ \\
hsPrp16 & 779 & $\mathrm{YSQ}$ \\
xIPrp16 & 783 & $\mathrm{YSQ}$ \\
cePrp16 & 689 & $\mathrm{YSQ}$ \\
ctPrp22 & 805 & $\mathrm{YSA}$ \\
scPrp22 & 731 & $\mathrm{YSA}$ \\
hsPrp22 & 813 & $\mathrm{YSA}$ \\
xtPrp22 & 807 & $\mathrm{YSA}$ \\
cePrp22 & 788 & $\mathrm{YGA}$
\end{tabular}
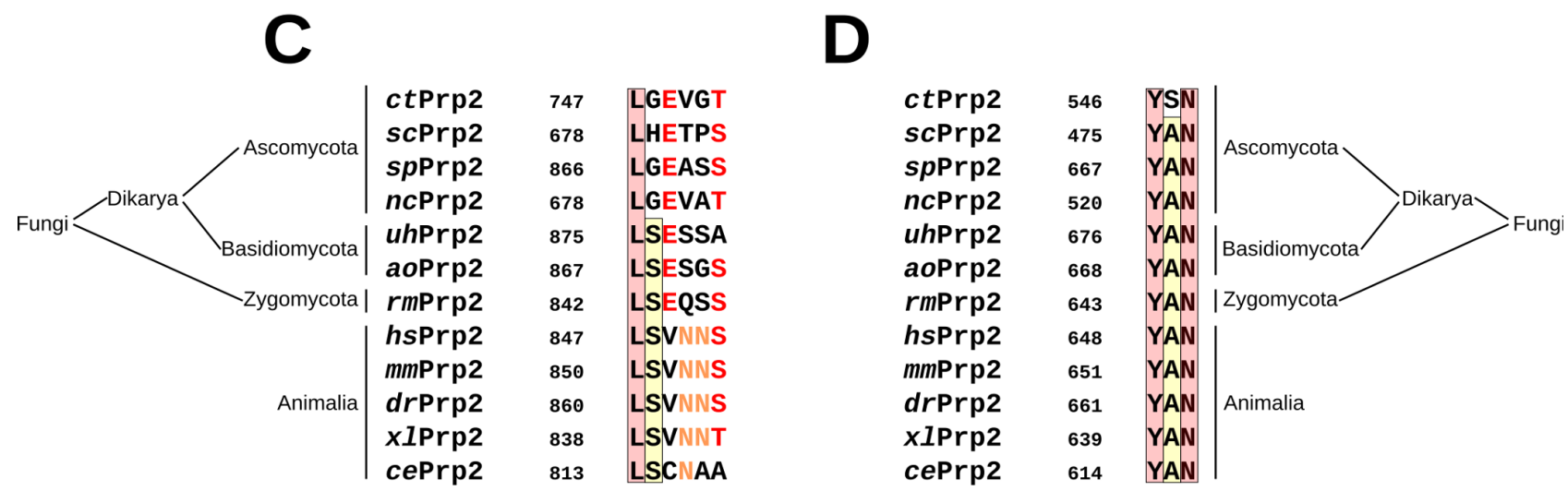

Figure 5: Sequence conservation of C-terminal loop and hook-loop. Sequence alignment of the C-terminal loop (A) and the hook-loop (B) of Prp2, Prp43, Prp16 and Prp22 from Chaetomium thermophilum, Saccharomyces cerevisiae, Homo sapiens, Xenopus laevis and Caenorhabditis elegans. Extended sequence alignment of the Prp2 C-terminal loop (C) and hook-loop (D) from Chaetomium thermophilum, Saccaromyces cerevisiae, Saccaromyces pombe, Neurospora crassa, Ustilago hordei, Armillaria ostoyae, Rhizopus microsporus, Homo sapiens, Mus musculus, Danio rerio, Xenopus laevis and Caenorhabditis elegans. 


\section{Supplementary information}

A

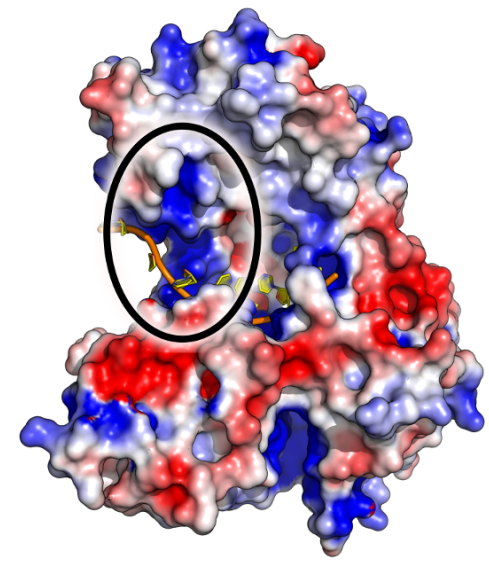

ctPrp2+ADP-BeF ${ }_{3}^{-}+\mathrm{RNA}^{-}$ (PDBid: $X X X X)$

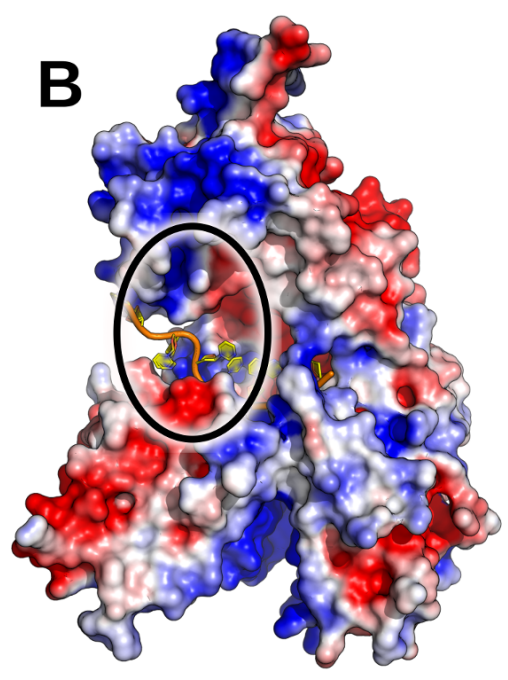

ctPrp43+ADP-BeF ${ }_{3}^{-}+\mathrm{RNA}^{-}$ (PDBid: 5lta)

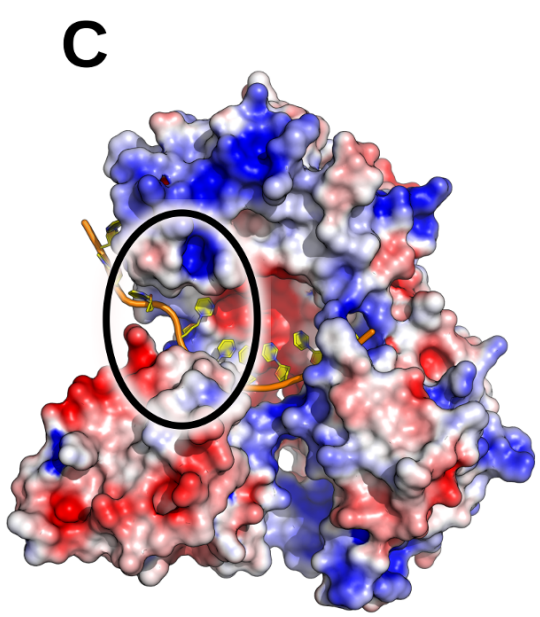

ctPrp22+RNA

(PDBid: 6i3p)

$$
+3.0 k_{B} T / e
$$

\section{$-3.0 k_{B} \mathrm{~T} / \mathrm{e}$}

Fig. S1: Electrostatic potential of RNA-bound Prp2, Prp43 and Prp22 structures. Prp2 (A), Prp43 (B) and Prp22 (C) are depicted as surface representation and colored based on their electrostatic potential. The RNA is displayed as a cartoon model. The region of interest close to the 5' RNA region kink is highlighted with a circle. The helix-bundle domain was omitted for clarity reasons. Prp2 exhibits the strongest positive charge of this region and likely influences the conformation of the 5' region. 
Chapter 4: Structural analysis of the ADP-BeF3-- and RNA-bound DEAH-box ATPase Prp2

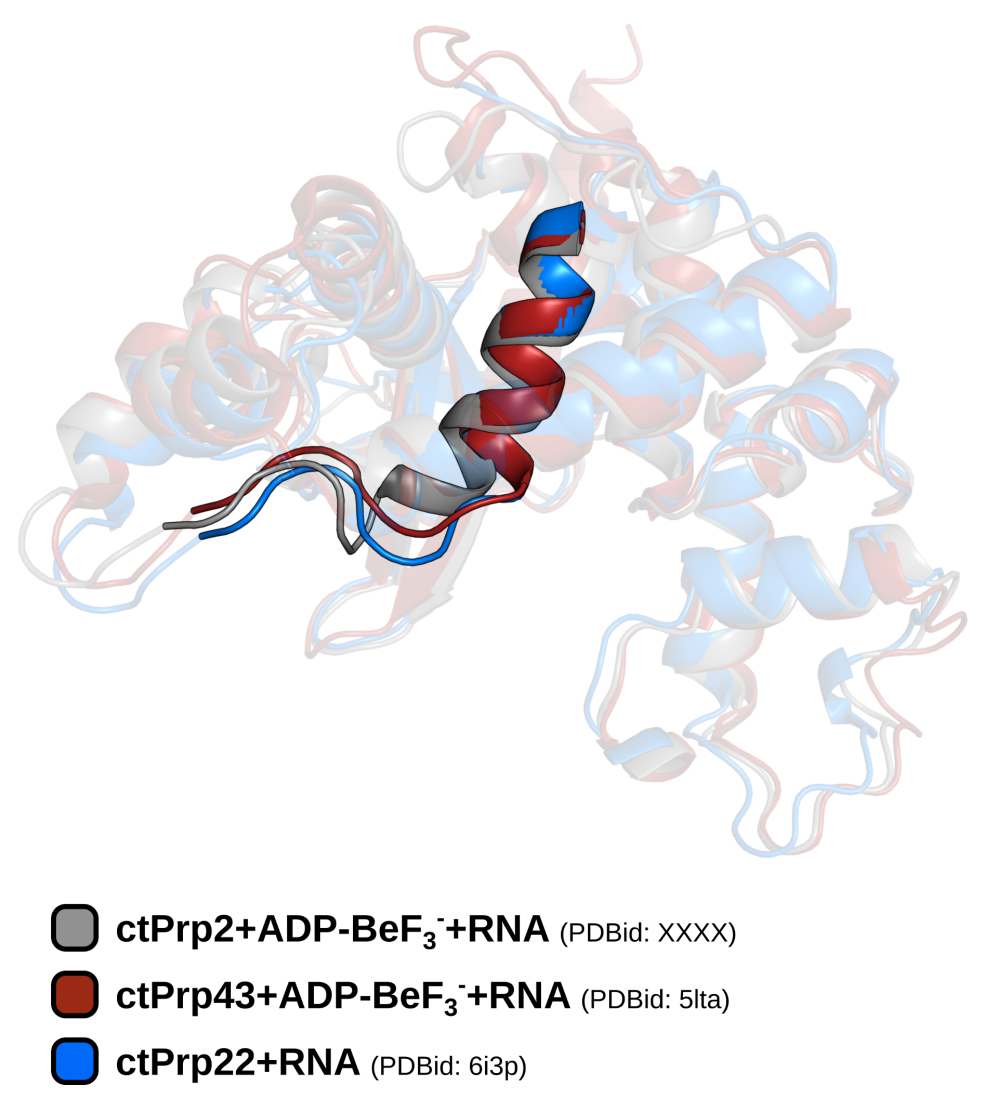

Fig S2: Comparison of the C-terminal loop of Prp2, Prp43 and Prp22. Due to the insertion in the Prp2 C-terminal loop, the helix proceeding this loop is significantly longer and the loop itself has a unique conformation. 


\title{
Chapter 5: Structural basis for RNA translocation by DEAH-box ATPases
}

This manuscript has originally been published in Nucleic Acids Research

\section{Structural basis for RNA translocation by DEAH-box}

\section{ATPases}

\author{
Florian Hamann ${ }^{1}$, Marieke Enders ${ }^{1}$, Ralf Ficner ${ }^{1 *}$
}

${ }^{1}$ Department of Molecular Structural Biology, Institute for Microbiology and Genetics, GZMB, GeorgAugust-University Göttingen, Justus-von-Liebig-Weg 11, 37077 Göttingen, Germany

*To whom correspondence should be addressed: rficner@gwdg.de

\section{Author contributions:}

F.H.: Experimental design, molecular cloning, protein expression and purification, crystallization, data collection and processing, structure determination, structure refinement and analysis, ATPase assays, RNAbinding assays, data analysis, preparation of figures/tables/movies, writing - original draft, writing - review and editing

M.E.: Molecular cloning, protein expression and purification, crystallization, data collection and processing, structure determination, structure refinement and analysis, circular dichroism spectroscopy

R.F.: Experimental design, crystal structure analysis, data analysis, writing - review and editing, supervision, project leader 


\title{
Structural basis for RNA translocation by DEAH-box ATPases
}

\author{
Florian Hamann, Marieke Enders and Ralf Ficner \\ Department of Molecular Structural Biology, Institute for Microbiology and Genetics, GZMB, Georg-August-University \\ Goettingen, Justus-von-Liebig-Weg 11, 37077 Goettingen, Germany
}

Received November 09, 2018; Revised January 28, 2019; Editorial Decision February 20, 2019; Accepted February 22, 2019

\begin{abstract}
DEAH-box adenosine triphosphatases (ATPases) play a crucial role in the spliceosome-mediated excision of pre-mRNA introns. Recent spliceosomal cryo-EM structures suggest that these proteins utilize translocation to apply forces on ssRNAs rather than direct RNA duplex unwinding to ensure global rearrangements. By solving the crystal structure of Prp22 in different adenosine nucleotide-free states, we identified two missing conformational snapshots of genuine DEAH-box ATPases that help to unravel the molecular mechanism of translocation for this protein family. The intrinsic mobility of the RecA2 domain in the absence of adenosine di- or triphosphate (ADP/ATP) and RNA enables DEAH-box ATPases to adopt different open conformations of the helicase core. The presence of RNA suppresses this mobility and stabilizes one defined open conformation when no adenosine nucleotide is bound. A comparison of this novel conformation with the ATP-bound state of Prp43 reveals that these ATPases cycle between closed and open conformations of the helicase core, which accommodate either a four- or five-nucleotide stack in the RNA-binding tunnel, respectively. The continuous repetition of these states enables these proteins to translocate in $3^{\prime}-5^{\prime}$ direction along an ssRNA with a step-size of one RNA nucleotide per hydrolyzed ATP. This ATP-driven motor function is maintained by a serine in the conserved motif $V$ that senses the catalytic state and accordingly positions the RecA2 domain.
\end{abstract}

\section{INTRODUCTION}

In eukaryotes, the removal of non-coding intron sequences in pre-mRNAs is performed by the spliceosome, a multimegadalton molecular machine composed of numerous RNA and protein components (1-3). The spliceosome is a highly dynamic complex in terms of conformation and com- position. All components are sequentially assembled in a stepwise manner and only a few of them remain constantly part of the complex. In order to remove one intron, the complex has first to be assembled mainly by the recruitment of five small nuclear ribonucleoprotein particles, named U1, $\mathrm{U} 2$, U4/U6 and $\mathrm{U} 5$, until reaching a preformed but catalytically inactive complex. After catalytic activation, two transesterification reactions ensure the excision of the intron and the ligation of the exons. Once this task is completed, the complex is completely disassembled and each component becomes available for another round of splicing.

The key triggering components of the spliceosome driving the transition from state to state are a set of so-called RNA helicases (4-6). The first assembly steps are governed by the DEAD-box helicases and the activated spliceosome is achieved by the action of the Ski2-like helicase Brr2. The catalytic activation, the catalysis itself as well as the final disassembly steps are driven by so-called DEAH-box helicases. They all belong to the helicase superfamily 2 (SF2) and share at least eight conserved sequence motifs (motifs I, Ia, Ib, II, III, IV, V and VI) spread over two RecA-like domains that together form the helicase core (7). These motifs are vital for the function of these proteins as they facilitate ATP and RNA binding, ATP hydrolysis as well as coupling ATP hydrolysis to RNA unwinding/translocation (811). Ski2-like and DEAH-box ATPases additionally possess auxiliary domains positioned C-terminally to the helicase core. Together they form a tunnel for single-stranded nucleic acid binding (12-14).

The release of the mature messenger RNA (mRNA) after intron cleavage is performed by the DEAH-box ATPase Prp22 $(15,16)$. It has multiple additional functions such as proof-reading, suppression of splicing of aberrant intermediates with suboptimal $3^{\prime}$ splice site and it participates in the second splicing reaction (17-19). Functionally Prp22 shares features common for other DEAH-box ATPases. They have the common ability to hydrolyze any kind of nucleoside triphosphates (NTPs) and the ATPase activity is stimulated by the presence of single-stranded RNA (ssRNA) $(11,20)$. They have no requirements for a specific RNA nucleotide sequence and generally unwind RNA duplexes with a $3^{\prime}$ overhang and with a $3^{\prime}-5^{\prime}$ polarity $(20,21)$.

\footnotetext{
${ }^{*}$ To whom correspondence should be addressed. Tel: +49 055139 14072; Fax: +49 055139 14082; Email: rficner@gwdg.de
} 


\section{Chapter 5: Structural basis for RNA translocation by DEAH-box ATPases}

DEAH-box ATPases have long been assigned as the key players responsible for enabling the sequential transition of the last spliceosomal stages. The primary function they have been attributed in the spliceosome is to induce conformational and compositional rearrangements of the different complexes by direct unwinding of RNA duplexes and have been termed helicases (16,22-24). While most of them indeed show in vitro helicase activity, none of the recent cryo-EM structures containing one of these so-called helicases shows an RNA duplex that could serve as a direct target (25-28). Instead, single-stranded RNA segments are usually spotted close by. The interaction of these proteins with spliceosomal RNA components is vital for their function and as direct duplex unwinding seems unlikely, the ATPases have to fulfill their tasks by other means. Disruption of duplexes in most SF2 members is tightly linked to processivity and while the spliceosomal context might not require DEAH-box members to be actual unwindases, translocation along a single-stranded RNA might still be needed. Due to their attachment to other protein factors at the spliceosomal peripheries, they are likely not mobile and do not translocate on long loose RNA stretches. Thus, translocation might enable the ATPases to apply forces on the RNA, a mechanism recently described as winching (19). This could not only ensure rearrangements in the vicinity of the ATPase, but these mechanical forces might as well be transmitted to more interior parts of the spliceosome potentially leading to indirect duplex disruptions. Thus, the sole translocation could be the primary function of DEAH-box ATPases in the spliceosome and a better understanding of the molecular mechanism of translocation might help to understand larger dynamic events of the spliceosome.

Although biochemical and genetic studies over the past decades and more recent structural cryo-EM studies have helped to locate and characterize DEAH-box ATPases in the context of the spliceosome, detailed mechanistic insights of how they function as a molecular machine are stil scarce $(11,21,25-30)$. Crystallographic approaches, mainly on Prp43, describing different catalytic states have started to deepen the understanding of how this protein family might work $(13,14,23,31,32)$. A key feature of these ATPases seems to be their conformational rearrangements that are strongly linked to their catalytic state. Thus, having structural snapshots of all possible catalytic states will help to fully explain their underlying molecular mechanism. Here, we present the first structures of the functional core of the spliceosomal DEAH-box ATPase Prp22 from Chaetomium thermophilum in two catalytic states previously undescribed for genuine DEAH-box ATPases that contribute to the completion of the catalytic state repertory. A Prp22 Apo structure highlights the mobility of the RecA2 domain in the absence of any adenosine nucleotide. A complex structure of $\operatorname{Prp} 22$ with a poly- $U_{9}$ ssRNA in the $a b-$ sence of ADP/ATP suggests that the RecA2 domain is stabilized by the interaction with the sugar-phosphate backbone of the RNA via a conserved and adenosine nucleotideindependent interaction pattern. The absence of ATP/ADP allows the helicase core to adopt an open conformation accommodating a one nucleotide longer RNA stretch in the binding tunnel. We identified a serine in motif $\mathrm{V}$ playing a crucial role in sensing the catalytic state and thereby reg- ulating the conformational dynamics of the helicase core. Comparing these new catalytic snapshots with known ones from Prp43, we propose a molecular model of the translocation mechanism of genuine DEAH-box ATPases based on a step-size of one RNA nucleotide per hydrolyzed ATP.

\section{MATERIALS AND METHODS}

\section{Protein production}

Prp22 from C. thermophilum (ctPrp22) was identified as an homolog of Saccharomyces cerevisiae Prp22 (scPrp22) using the NCBI BLAST search tool (GenBank: EGS21698.1, 33). An N-terminally truncated version missing the first 545 residues was amplified by polymerase chain reaction from a total DNA preparation. The amplified DNA product was cloned into pGEX-6p1 vector using the EcoRI and NotI restriction sites. The forward primer contained the sequence for the Tobacco Etch Virus (TEV) cleavage site directly prior to the etPrp22 construct. The GST fusion protein was recombinantly expressed in Rosetta 2 (DE3) cells using an autoinduction protocol (34). Harvested cells were disrupted using a microfluidizer (Microfluidics) in $50 \mathrm{mM}$ Tris/ $\mathrm{HCl}(\mathrm{pH} 7.5), 500 \mathrm{mM} \mathrm{NaCl}, 5 \%(\mathrm{v} / \mathrm{v})$ glycerol and $10 \mathrm{mM}$ ethylenediaminetetraacetic acid (EDTA). The lysate was clarified via ultracentrifugation at $30000 \mathrm{~g}$ and $4^{\circ} \mathrm{C}$ for $30 \mathrm{~min}$. The clarified lysate was loaded on a Glutathione Sepharose column (GE Healthcare) at $20^{\circ} \mathrm{C}$. Potentially bound nucleic acids were removed using a wash step with lysate buffer supplemented with $2 \mathrm{M} \mathrm{LiCl}$ and the protein was subsequently eluted with $30 \mathrm{mM}$ reduced glutathione. The GST-tag was proteolytically cleaved with 1:50 (w/w) TEV protease overnight at $4{ }^{\circ} \mathrm{C}$. Remaining impurities and the tag were removed using a Superdex 75 gel-filtration column coupled to a Glutathione Sepharose column in $20 \mathrm{mM}$ Tris/HCl (pH 7.5), $200 \mathrm{mM} \mathrm{NaCl}, 5 \%$ glycerol and $2 \mathrm{mM}$ $\mathrm{MgCl}_{2}$. The protein was concentrated to $7 \mathrm{mg} \mathrm{ml}^{-1}$ with an Amicon Ultra centrifugal concentrator (Merck). About 200 $\mu \mathrm{l}$ protein samples were flash frozen in liquid nitrogen and stored at $-80^{\circ} \mathrm{C}$. ctPrp43, ctPrp43-S387A, ctPrp43-S387G, ctPrp22-S837A, ctPrp22-S837G and ctPrp22-S837P were expressed and purified using the same protocol as described for ctPrp22.

\section{Crystallization}

ctPrp22 and ctPrp22-S837A were diluted to $2.5 \mathrm{mg} \mathrm{ml}^{-1}$ $(32.76 \mu \mathrm{M})$ and incubated with a 10 -fold molar excess of ADP, a 20-fold molar excess of $\mathrm{BeSO}_{4}$, a 60 -fold molar excess of $\mathrm{NaF}$ and a 2.5 -fold molar excess of $\mathrm{U}_{12}$-RNA (AXOlabs, Germany) for at least $30 \mathrm{~min}$ at $4^{\circ} \mathrm{C}$. Crystallization was performed via the sitting-drop vapor-diffusion technique by mixing $1 \mu 1$ complex solution with $1 \mu 1$ crystallization buffer. ctPrp22 Apo crystals were obtained in 100 $\mathrm{mM}$ Bis-Tris propane ( $\mathrm{pH}$ 6.5), $400 \mathrm{mM}$ K-Thiocyanate and 20\% (w/v) PEG3350 and ctPrp22+RNA and ctPrp22$\mathrm{S} 837 \mathrm{~A}+\mathrm{RNA}$ crystals in $100 \mathrm{mM}$ Bis-Tris propane $(\mathrm{pH}$ 8.5), $250 \mathrm{mM} \mathrm{NaF}$ and $22 \%$ (w/v) PEG3350. All crystals were obtained after $2-3$ days at $20^{\circ} \mathrm{C}$. ctPrp43-S837A and ctPrp43-S387G crystals were obtained as described in Tauchert et al. (14). 


\section{Chapter 5: Structural basis for RNA translocation by DEAH-box ATPases}

\section{Data collection and processing}

Crystals of ctPrp22 and ctPrp22-S837A were cryoprotected in the respective reservoir solution complemented with $10 \%$ (w/v) PEG400 and 5\% (w/v) glycerol and flash-cooled in liquid nitrogen prior to data collection. Crystals of ctPrp43S387A and ctPrp43-S387G were not additionally cryoprotected prior to flash-cooling. X-ray diffraction data were collected at $100 \mathrm{~K}$ on beamline P13 (ctPrp22) and beamline P14 (ctPrp22-S837A and ctPrp43-S387A), PETRA III, DESY (Hamburg, Germany), as well as on Rigaku MicroMax-003 equipped with a Dectris Pilatus $2 \mathrm{~K}$ detector (ctPrp43-S387G). The XDS package was used for data processing. The highest resolution limit was estimated using a minimum $\mathrm{I} / \sigma(\mathrm{I})$ of 1.5 and a minimum $\mathrm{CC}_{1 / 2}$ of $60 \%$ as cutting criteria (35).

\section{Structure solution, refinement and analysis}

The ctPrp22+RNA complex structure was solved by molecular replacement using Phaser with the structure of ctPrp2 (PDB ID: 6fa5) used as a search model $(36,37)$. Prior to molecular replacement, the amino acid sequence of the ctPrp2 model was modified to match the sequence of ctPrp22 using the chainsaw utility in the CCP4 suite (38). Due to the different conformations of ctPrp22 in the presented structures, no molecular replacement solution could be obtained with this preliminary model. After splitting it into three domains (RecA1, RecA2 and C-terminal) and using them as subsequent search ensembles for molecular replacement, a solution was found. The structure of ctPrp22-Apo was solved using the same individual domains as search ensembles but from the final model of the ctPrp22+RNA complex. ctPrp22 Apo contains two molecules and ctPrp22+RNA four molecules per asymmetric unit. The models were manually built with Coot and refinements were performed with Refmac5 $(39,40)$. Both structures were refined using the RecAl, RecA2 and Cterminal domains as translation, libration and screw-axis (TLS) groups and with the inclusion of automatically generated local non-crystallographic symmetry (NCS) restraints (41). The ctPrp22-Apo structure was additionally refined with external restraints using the ctPrp22 model from the RNA complex as a reference structure (42). Serine mutant structures of ctPrp22 and ctPrp43 were solved using the corresponding wild-type structure (ctPrp22-S837A: 6i3p; ctPrp43-S387A and ctPrp43-S387G: 51tj) for molecular replacement. The quality of the final model was assessed using MolProbity (43). Structure superpositions were calculated with $L S Q M A N$ (44). Figures were prepared with $P y M O L$ (v.1.8, Schrödinger) and Chimera (45)

\section{ATPase activity assay}

The ATPase activity measurements of ctPrp22 and ctPrp43 were conducted with a nicotinamide adenine dinucleotide (NADH) dependent coupled enzymatic assay (46). The decrease of the NADH absorption at $340 \mathrm{~nm}$ as a direct effect of the ATP consumption was recorded over time with a VICTOR Nivo Multimode Microplate Reader (PerkinElmer). All reactions were performed in triplicates of $150 \mu \mathrm{l}$ each at $25^{\circ} \mathrm{C}$ in $25 \mathrm{mM}$ Bis-Tris propane ( $\mathrm{pH} 7.5$ ),
$150 \mathrm{mM} \mathrm{KCl}$ and $3 \mathrm{mM} \mathrm{MgCl} 2$ supplemented with $250 \mathrm{nM}$ NADH, $500 \mathrm{nM}$ phosphoenolpyruvate, $6-8.3 \mathrm{U} \mathrm{ml}^{-1}$ pyruvate kinase and 9-14 $\mathrm{U} \mathrm{ml}^{-1}$ lactic dehydrogenase. The ATP concentration was varied from 0 to $2 \mathrm{mM}$. Measurements in the presence of RNA were conducted with a 20 -fold molar excess over ctPrp22 or ctPrp43 of a $\mathrm{A}_{20}$-ssR NA (AXOlabs). ctPrp22 was used at a concentration of $0.4 \mu \mathrm{M}$ and ctPrp43 at $5 \mu \mathrm{M} . K_{\mathrm{m}}$ and $k_{\mathrm{cat}}$ were calculated by fitting the experimental data according to the Michaelis and Menten equation using QtiPlot (v.0.9.8.9)

\section{RNA binding assay}

The RNA binding in dependence of different adenosine nucleotides was performed via fluorescence polarization measurements using a VICTOR Nivo Multimode Microplate Reader (PerkinElmer). The binding of $6 \mathrm{nM} 3^{\prime}$ 6carboxyfluorescein-labeled $\mathrm{A}_{20}$-RNA to up to $70 \mu \mathrm{M}$ ctPrp22 and $120 \mu \mathrm{M}$ ctPrp43 was monitored in $20 \mathrm{mM}$ Tris/ $\mathrm{HCl}$ (pH 7.5), $200 \mathrm{mM} \mathrm{NaCl}, 5 \%$ glycerol and $3 \mathrm{mM}$ $\mathrm{MgCl}_{2}$. Measurements in presence of ADP/adenylyl imidodiphosphate (AMPPNP) were performed with a 50-fold molar excess of the nucleotide over ctPrp22 or ctPrp43, except for ADP-dependent measurements of ctPrp22 where a constant concentration of $3.5 \mathrm{mM}$ of the nucleotide was used throughout all measurements. All reactions were performed in $50 \mu \mathrm{l}$ and measured as a set of triplicates. For all measurements an excitation wavelength of $480 \mathrm{~nm}$ was set and the emission was detected at $530 \mathrm{~nm}$ for $500 \mathrm{~ms}$. The measured fluorescence polarization was normalized and fitted according to Rossi and Taylor (47) using QtiPlot (v.0.9.8.9).

\section{Circular dichroism spectroscopy}

In order to verify the structural integrity of ctPrp22-S837P, far-UV spectra $(185-260 \mathrm{~nm})$ of this mutant protein and the wild-type protein were recorded using a Chirascan CD spectrometer (Applied Photophysics). Measurements were performed in $20 \mathrm{mM}$ Na-phosphate buffer at $20^{\circ} \mathrm{C}$ with a protein concentration of $0.1 \mathrm{mg} \mathrm{ml}^{-1}$. For each construct, 15 spectra of $1 \mathrm{~s}$ accumulation were averaged for the final spectrum. The CD data are presented as mean residue ellipticity $(\theta)$ and fitted using QtiPlot (v.0.9.8.9) (48).

\section{RESULTS AND DISCUSSION}

\section{Prp22 architecture and function}

The crystallographic structure determination of the spliceosomal DEAH-box ATPase Prp22 presented here was performed with the protein from the ascomycete $C$. thermophilum (ctPrp22). Previous studies on other closely related spliceosomal DEAH-box members, Prp2 and Prp43, have demonstrated that proteins from this organism are suited for crystallographic investigations as they might have an increased tendency to crystallize when compared with orthologs from $S$. cerevisiae or Homo sapiens $(14,32,37,49)$. ctPrp22 exhibits an amino acid sequence identity of 54.7 and $57.7 \%$ to yeast and human $\operatorname{Prp} 22$, respectively. As the $\mathrm{N}$-terminal region of Prp22 is predicted to be in part unfolded and recent structural studies on Prp2 and Prp43 have 


\section{Chapter 5: Structural basis for RNA translocation by DEAH-box ATPases}

proven that the helicase core and the C-terminal domains are the key domains involved in the function of this ATPase family, an N-terminally truncated version of ctPrp22 lacking the first 545 amino acids was used for structure determination (Supplementary Figure S1A). The construct lacking the N-terminus has a sequence identity of 65.2 and $73.5 \%$ to yeast and human Prp22, respectively. Two different crystal structures of ctPrp22 were obtained, one with a bound single-stranded RNA and another one with no interaction partner (Table 1).

As in the used construct the N-terminal region, which contains the S1 motif in Prp22, has been completely omitted, only five of the six DEAH-box specific domains are present (50). Two RecA-like domains, referred to as RecAl and RecA2, build up the helicase core (Figure 1A). These domains harbor the eight conserved sequence motifs known to play a crucial role in ATP and RNA binding as well as in coupling ATP hydrolysis to translocation/RNA unwind ing. Apart from these conserved sequence motifs, recent studies on Prp43 and the closely related DExH-box helicase maleless (MLE) have identified additional structural elements, as the hook-loop and hook-turn, which are crucial for the function of these helicases $(14,51)$. The RecA2 domain contains an anti-parallel $\beta$-hairpin present in all DExH-box helicases, which protrudes out of the RecA2 domain thereby contacting the $\mathrm{C}$-terminal domains. These domains are divided in three distinct domains, namely the winged-helix (WH), the helix-bundle (HB) and the oligonucleotide-binding $(\mathrm{OB})$ domain. These three domains are arranged on top of the helicase core thereby forming an RNA-binding tunnel between these domains. Upon binding of ATP, this tunnel is exposed and the WH domain serves as a hinge region for the opening of the tunnel (14). Interestingly, the C-terminal 47 amino acids were not traceable in the electron density map and thus are missing in the model of ctPrp22, suggesting a high flexibility of this C-termina tail. In the most recently published cryo-EM structure of the postcatalytic P complex, the major part of this region could be built, probably due to numerous interactions with other spliceosomal factors and its integration in more inner parts of the complex (25). The global architecture of Prp22 strongly resembles the one already known from Prp2 (PDB ID: 6fa5) and Prp43 (PDB ID: 5lta) (14,37).

The N-terminally truncated $\operatorname{Prp} 22$ construct is a competent ATPase and similar to other DEAH-box ATPases it is stimulated by the presence of RNA (Figure 1B) $(29,52,53)$. The degree of stimulation by a single-stranded $\mathrm{A}_{20}-\mathrm{RNA}$ is strongly dependent on the excess given to the ATPase reaction, exhibiting up to a 3.5 -fold stimulation of $k_{\text {cat }}$ and a linear increase of $k_{\mathrm{cat}}$ and $K_{\mathrm{m}}$ using up to 40 -fold molar excess of ssRNA over protein (Supplementary Figure S1b and c). In contrast to previous findings, reporting that the stimulation by RNA only influences the $k_{\text {cat }}$, our data shows an increase of both, $k_{\text {cat }}$ and $K_{\mathrm{m}}$ (Figure 1B) (21). This discrepancy might be due to the lack of the N-terminal region of the used construct or Prp22 from C. thermophilum functions in a different manner than yeast Prp22 in this regard. Prp22 binds ssRNA in absence as well as in presence of adenosine nucleotide (Figure 1C). However, in the presence of the nonhydrolysable ATP-analog AMPPNP, the binding is slightly more efficient than with no adenosine nucleotide present.
The binding of ssRNA is significantly decreased in the presence of ADP. Recent structural studies on ctPrp43 indicate that the ssRNA is loaded via an ATP-mediated opening of the RNA-binding tunnel and the increased affinity in presence of AMPPNP is in agreement with these findings (14). Nevertheless, DEAH-box ATPases seem to be able to bind ssRNA also in the absence of ATP, suggesting the opening of the tunnel is not strictly dependent on the binding of ATP but is less efficient without (Figure 1C).

Prp43 is by far the most biochemically and structurally studied spliceosomal DEAH-box ATPase and to ensure mechanistic conclusions derived from structural comparisons in upcoming chapters are legitimate, a comparably truncated version of ctPrp43 (61-764) was subjected to the same basic biochemical characterization. Although $k_{\text {cat }}, K_{\mathrm{m}}$ and $K_{\mathrm{d}}$ values deviate from the ones measured for Prp22, both ATPases are stimulated by the presence of RNA and the binding of ssRNA in dependence of the adenosine nucleotide exhibits the same tendencies (Figure 1B and C). Discrepancies in the magnitude of the measured values might arise from the fact that Prp43 depends on the interaction with so called G-patch proteins (Pfa1, Ntr1, Gnol and Cmg1), which generally have a stimulating effect on Prp43 $(22,54,55)$. Since $\operatorname{Prp} 22$ is not known to require a regulatory G-patch protein and has already elevated activity, all biochemical studies as well as structural comparisons were conducted without the consideration of G-patch proteins. Altogether, these findings suggest that these two ATPases share a common mechanism of interacting with ssRNAs, which can be likely applied to the rest of the DEAH-box members, and justifies following structural comparisons of catalytic states of these two ATPases.

\section{RNA binding of Prp22}

For the crystallization of the complex structure of ctPrp22 with a single-stranded RNA, a $\mathrm{U}_{12}$-RNA was used, of which nine nucleotides were traceable in the electron density map (Figure 2A). As already reported for other members of the DExH-box family, the ssRNA binds in an RNAbinding tunnel which is formed between the helicase core and the C-terminal domains $(14,51,56,57)$. In contrast to the closely related DExH-box helicases, DHX36 and MLE, which exhibit a preference toward G-rich and U-rich sequences, respectively, genuine DEAH-box ATPases interact with RNA in a sequence-unspecific manner, as has been shown for Prp43 (14). In the Prp22-RNA complex structure, the ssRNA is also predominantly bound via polar interactions with the sugar-phosphate backbone (Figure 2A). The only exceptions are $U_{9}$, as the base forms hydrogenbond interactions with the side chains of N919 and R660, and the bases of $\mathrm{U}_{1}-\mathrm{U}_{3}$ that interact via $\pi-\pi$-stacking with F1134 and cation $-\pi$ stacking with R1012 (Figure 2C). The latter interactions are feasible with any base and thus do not determine sequence-specificity.

Amino acids interacting with the sugar-phosphate backbone of RNA-nucleotides $\mathrm{U}_{5}-\mathrm{U}_{9}$ are highly conserved as they are part of the conserved eight sequence motifs of DEAH-box proteins or the recently proposed structural elements hook-loop and hook-turn (Supplementary Figure S2a). Residues R612 (motif Ia), R639 (hook-turn, HT), 


\section{Chapter 5: Structural basis for RNA translocation by DEAH-box ATPases}

Table 1. Data collection and refinement statistics

\begin{tabular}{|c|c|c|c|c|c|}
\hline Data collection & ctPrp22 Apo & $\begin{array}{l}\text { ctPrp22 } \\
+ \text { RNA }\end{array}$ & $\begin{array}{l}\text { ctPrp22-S837A } \\
+ \text { RNA }\end{array}$ & $\begin{array}{l}\text { ctPrp43-S387A } \\
+\mathrm{ADP}_{-} \mathrm{BeF}_{3}{ }^{-} \\
\end{array}$ & $\begin{array}{l}\text { ctPrp43-S387G } \\
+\mathrm{ADP}_{-} \mathrm{BeF}_{3}{ }^{-} \\
\end{array}$ \\
\hline Space group & $\mathrm{P} 2{ }_{1} 2_{1} 2_{1}$ & $\mathrm{P} 2{ }_{1} 2_{1} 2_{1}$ & $\mathrm{P} 22_{1} 2_{1} 2_{1}$ & $\mathrm{P} 2_{1} 2_{1} 2_{1}$ & $\mathrm{P} 22_{1}{ }_{1} 2_{1}$ \\
\hline $\mathrm{a}(\AA)$ & 67.5 & 139.8 & 139.6 & 88.8 & 88.6 \\
\hline $\mathrm{b}(\AA)$ & 93.6 & 140.5 & 140.0 & 105.5 & 103.0 \\
\hline $\mathrm{c}(\AA)$ & 218.4 & 159.6 & 164.7 & 119.2 & 118.9 \\
\hline $\mathrm{X}$-ray source & $\begin{array}{l}\text { P13, PETRA III, } \\
\text { DESY }\end{array}$ & $\begin{array}{l}\text { P13, PETRA III, } \\
\text { DESY }\end{array}$ & $\begin{array}{l}\text { P14, PETRA III, } \\
\text { DESY }\end{array}$ & $\begin{array}{l}\text { Rigaku, } \\
\text { MicroMax-003 }\end{array}$ & $\begin{array}{l}\text { P14, PETRA III, } \\
\text { DESY }\end{array}$ \\
\hline Resolution range $(\AA)$ & $\begin{array}{l}86.06-3.25 \\
(3.35-3.25)\end{array}$ & $\begin{array}{l}49.24-2.75 \\
(2.87-2.75)\end{array}$ & $\begin{array}{l}106.49-2.70 \\
(2.82-2.70)\end{array}$ & $\begin{array}{l}48.22-2.20 \\
(2.30-2.20)\end{array}$ & $\begin{array}{l}77.85-2.70 \\
(2.80-2.70)\end{array}$ \\
\hline No. of unique reflections & 22460 & 82130 & 88822 & 57004 & 30459 \\
\hline Completeness $(\%)$ & $99.5(96.3)$ & $99.9(100.0)$ & $99.7(99.8)$ & $99.1(93.0)$ & $99.8(99.8)$ \\
\hline$R_{\text {merge }}(\%)$ & $11.3(106.0)$ & $11.0(146.9)$ & $7.7(147.4)$ & $6.6(56.6)$ & $11.3(147.4)$ \\
\hline Average I/ $\sigma(\mathrm{I})$ & $12.04(1.52)$ & $14.28(1.51)$ & $19.56(1.69)$ & $20.81(2.51)$ & $14.78(1.64)$ \\
\hline Redundancy & $5.20(4.98)$ & $7.79(8.15)$ & $9.25(9.44)$ & $7.47(4.20)$ & $9.11(9.24)$ \\
\hline $\mathrm{CC}_{1 / 2}$ & $99.8(65.7)$ & $99.9(75.0)$ & $100.0(70.5)$ & $99.9(81.9)$ & $99.9(64.8)$ \\
\hline Wilson $\mathrm{B}\left(\AA^{2}\right)$ & 76.35 & 72.84 & 81.23 & 36.44 & 71.31 \\
\hline \multicolumn{6}{|l|}{ Refinement } \\
\hline Resolution $(\AA)$ & $\begin{array}{l}86.06-3.25 \\
(3.36-3.25)\end{array}$ & $\begin{array}{l}49.24-2.75 \\
(2.82-2.75)\end{array}$ & $\begin{array}{l}106.49-2.70 \\
(2.77-2.70)\end{array}$ & $\begin{array}{l}35.61-2.20 \\
(2.24-2.20)\end{array}$ & $\begin{array}{l}77.85-2.70 \\
(2.79-2.70)\end{array}$ \\
\hline No. of reflections & 21337 & 78023 & 84380 & 56985 & 30443 \\
\hline$R_{\text {work }}(\%)$ & $24.65(36.6)$ & $22.21(37.6)$ & $21.22(37.6)$ & $18.36(27.0)$ & $22.86(42.9)$ \\
\hline$R_{\text {free }}(\%)$ & $26.06(40.1)$ & $25.23(39.8)$ & $26.15(38.6)$ & $20.81(28.3)$ & $25.37(46.9)$ \\
\hline $\begin{array}{l}\text { Molecules per asymmetric } \\
\text { unit }\end{array}$ & 2 & 4 & 4 & 1 & 1 \\
\hline Total number of atoms & 9208 & 20540 & 20594 & 6472 & 5741 \\
\hline Protein residues & 1166 & 2509 & 2510 & 703 & 703 \\
\hline Water molecules & / & 1 & 1 & 704 & 39 \\
\hline $\begin{array}{l}\text { Nucleic acid molecules } \\
\text { r.m.s. deviations }\end{array}$ & / & 4 & 4 & / & / \\
\hline Bond length $(\AA)$ & 0.012 & 0.011 & 0.009 & 0.009 & 0.010 \\
\hline Bond angles $\left({ }^{\circ}\right)$ & 1.74 & 1.56 & 1.51 & 0.91 & 0.97 \\
\hline \multicolumn{6}{|l|}{ Mean B-factors $\left(\AA^{2}\right)$} \\
\hline Protein & 112.34 & 78.48 & 93.32 & 34.99 & 77.09 \\
\hline RNA & / & 81.23 & 101.30 & / & / \\
\hline \multicolumn{6}{|l|}{ Ramachandran statistics } \\
\hline Favored $(\%)$ & 95.01 & 97.03 & 96.55 & 97.72 & 98.00 \\
\hline Allowed $(\%)$ & 4.90 & 2.81 & 3.45 & 2.28 & 2.00 \\
\hline Outliers $(\%)$ & 0.09 & 0.16 & 0.00 & 0.00 & 0.00 \\
\hline PDB ID & $6 \mathrm{i} 30$ & $6 i 3 p$ & 6qic & 6qid & 6qie \\
\hline
\end{tabular}

T654 (motif Ib), T831 (motif V), S837 (motif V) and Q1073 (HB domain) are strictly conserved among DExH-box helicases Prp22, Prp43, MLE and DHX36. K853 ( $\beta$-hairpin) is replaced by an arginine in MLE still allowing the same type of interaction, S806 (hook-loop, HL) is substituted by a glycine in Prp43 that still interacts with the RNA via the mainchain and N832 is exchanged to an isoleucine in DHX36 from Drosophila melanogaster $(14,51,56,57)$ These conserved interactions lead in all structures to a stacked conformation of the RNA in the $3^{\prime}$ region, with the bases pointing toward the $\mathrm{C}$-terminal domains (Figures $2 \mathrm{~A} ; 4 \mathrm{~A}$ and $\mathrm{B})$.

In contrast to the highly conserved backbone interactions and the stacked RNA conformation at the $3^{\prime}$ end, the $5^{\prime}$ end of ssRNAs in complex with DExH-box ATPases exhibits more variability in terms of number of interactions and conformation (Supplementary Figure S2c). While the $5^{\prime}$ end of the RNA only loosely associates with ctPrp43 leading to alternative conformations, the same region of the RNA is highly stabilized in ctPrp 22 mainly by stacking interactions with residues of the $\mathrm{HB}$ and OB-fold domains (Figure 2B and C) (14). The thorough analysis of the Prp22-RNA complex structure reveals a novel RNAbinding motif present in the OB-fold domain, which in- volves H1128, P1129 and F1134 as a stacking triad. H1128 directly interacts via hydrogen-bonding with the phosphate of $\mathrm{U}_{4}$ and the ribose of $\mathrm{U}_{3}$, but also undergoes stacking with P1129, which itself stacks with F1134 (Figure 2C). This last residue of the stacking triad $\pi-\pi$-stacks in a paralleldisplaced manner with the $U_{1}$ base as well as in an edgeto-face fashion with the $\mathrm{U}_{2}$ base. Additionally, a further cation $-\pi$ interaction between the $\mathrm{U}_{3}$ base and $\mathrm{R} 1012$ of the HB domain and hydrogen-bonds between the sugarphosphate backbone with T1149 and K1151 of the OB-fold contribute to the stabilization of the $5^{\prime}$ end. All these interactions lock the $5^{\prime}$ end of the RNA into a conformation where the bases point into the opposite direction of the $\beta$ hairpin of the RecA2 domain (Figure 2B). This is particularly interesting as the $\beta$-hairpin has been suggested to be involved in double-strand separation based on the structural comparison with the Ski2-like helicase Hel308 $(12,23,31)$. By orienting the bases of the most $5^{\prime}$ RNA nucleotides away from the $\beta$-hairpin, Prp22 likely positions a potential double-strand on the opposite side of this structural feature. This finding is not in agreement with the proposed role of the $\beta$-hairpin as it does not seem to act as a strand separator in Prp22. 

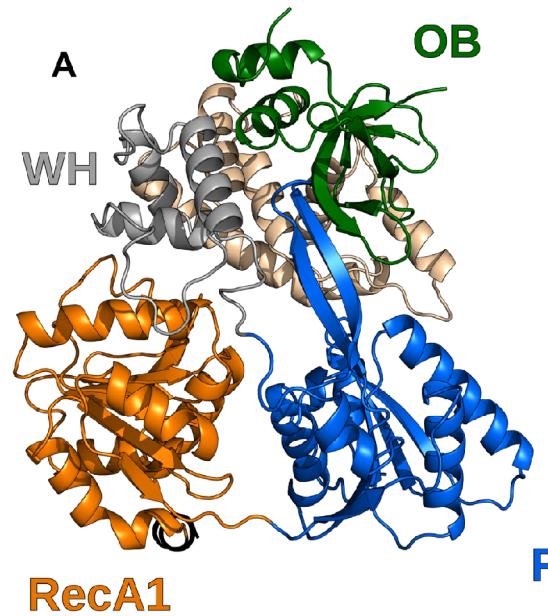

\section{RecA2}

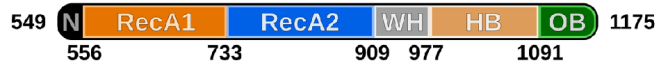

\section{N-Terminus}
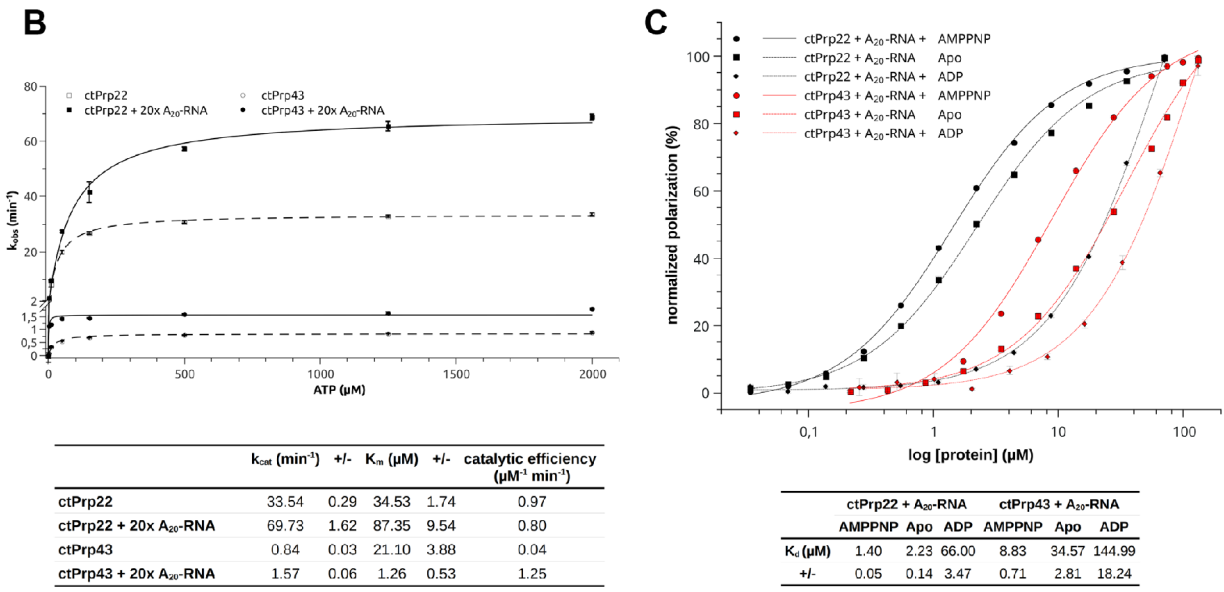

Figure 1. Structural overview of Prp22 from Chaetomium thermophilum and functional comparison of ctPrp22 and ctPrp43. (A) Structural overview of Prp22 from C. thermophilum. The model of ctPrp22 is displayed as a cartoon representation. Residues from the truncated N-terminus (549-556) are shown in black, the RecA1 domain (557-733) in orange, the RecA2 domain (734-909) in blue, the WH domain (WH; 910-977) in gray, the HB domain (HB; 978-1091) in wheat and the OB fold (OB; 1092-1175) in green. (B) ATPase assays show that ctPrp22 has an increased ATPase activity compared to ctPrp43, but both are stimulated by the presence of an $\mathrm{A}_{20}$-RNA. (C) ctPrp22 and ctPrp43 show the same binding mode toward an $\mathrm{A}_{20}$-RNA in dependence of an adenosine nucleotide. Both bind the ssR NA best in presence of AMPPNP and show less binding in absence of any adenosine nucleotide. The affinity toward ssRNA is drastically decreased when ADP is present. ATPase activity as well as RNA-binding experiments were determined in triplicates and error bars for each measured data point are depicted. The error of fit is indicated in the tables as $+/-$.

Spliceosomal cryo-EM structures containing DEAH-box ATPases show that the $\mathrm{C}$-terminal domain of these proteins are the major anchor for the interaction with the spliceosomal complex $(25,28,58-61)$. Especially the OB-fold domain is majorly involved in contacts with other spliceosomal factors and is always facing toward the core of the complexes. This orientation of the ATPases relative to the rest of the complex makes sense as the entrance of the RNA-binding tunnel is already properly positioned for the incorporation of the ssRNA. Here, the stacking triad could help to bridge the distance from the spliceosomal complex to the helicase core of the DEAH-box ATPase and efficiently guide the ssRNA into the binding tunnel. Additionally, during the process of winching, translocation of the DEAH-box proteins is thought to apply pulling forces on the ssRNA and in this case the stacking triad might act as a kind of safety-lock that prevents backlashing of the RNA.

Intriguingly, the histidine and the proline of the stacking triad are strictly conserved among all genuine DEAH-box ATPases, while the phenylalanine is less conserved (Sup- 


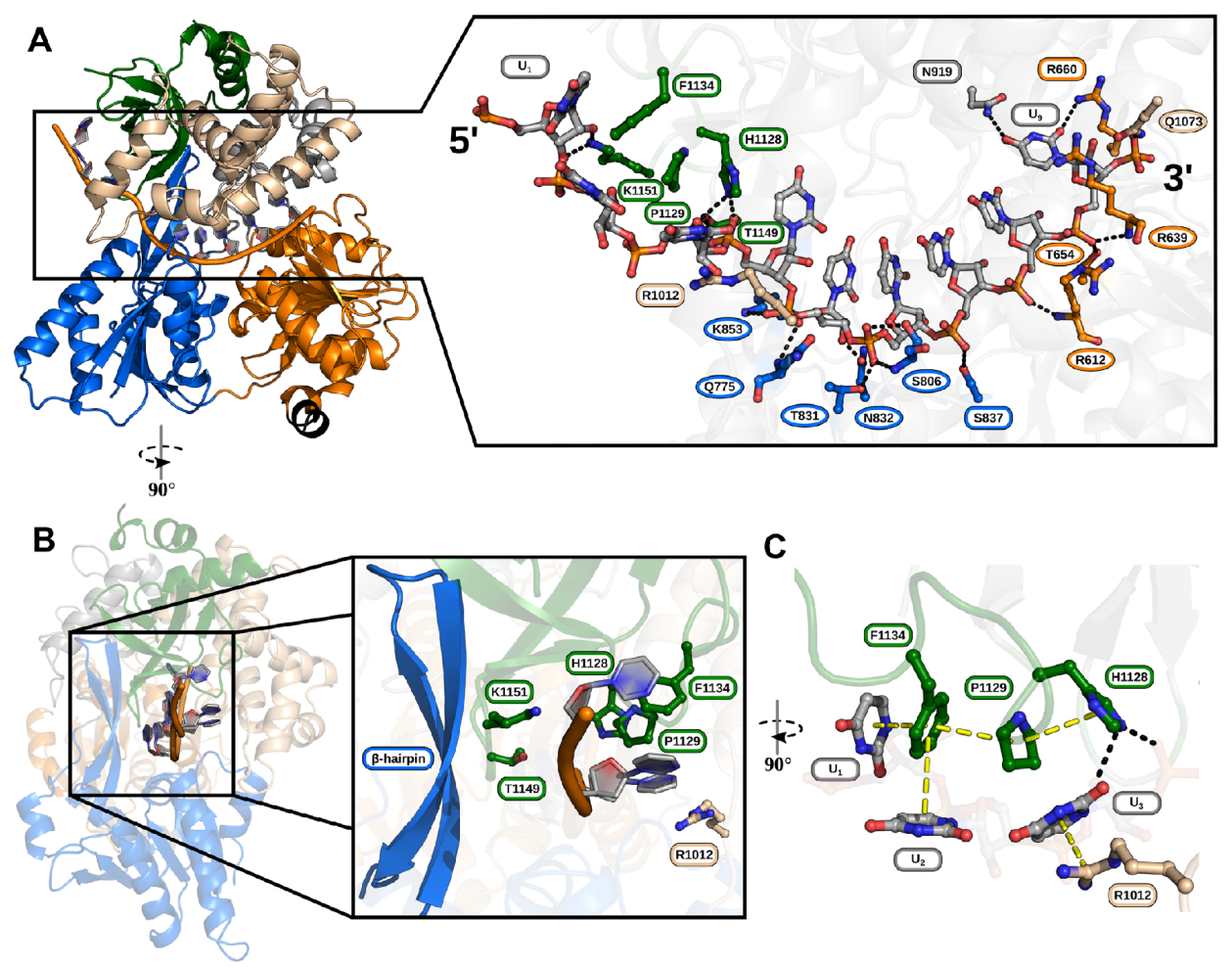

Figure 2. RNA interactions of ctPrp22. (A) The ssRNA binds in the RNA-binding tunnel between helicase core and C-terminal domains. The RNA and amino acids either hydrogen-bonding or stacking with nucleotides of the RNA are depicted as ball and stick models and colored according to the domain coloring of Figure 1. The numbering of residues exhibiting the conserved RNA-backbone interaction pattern with the $3^{\prime}$ region as seen as well in the ctPrp43+ADP-BeF $3^{-}+\mathrm{RNA}$ structure (PDB ID: 5lta) is highlighted as ovals, whereas residues displaying a unique interaction have a rectangular shape. (B) Overview of $5^{\prime}$ interactions leading to the unique conformation of this region. Bases of $\mathrm{U}_{1}-\mathrm{U}_{3}$ point in the opposite direction of the $\beta$-hairpin. Major
(B) contributors to this conformation are residues H1128, P1129 and F1134 of the OB-fold domain forming a stacking triad and R1012 from the HB domain (C).

plementary Figure S2b). In Prp22 from S. cerevisiae, it is replaced by a tyrosine and in Prp2 and Prp16 also a histidine or arginine can be found at this position, all of which could still support stacking. Prp43 is the only spliceosomal DEAH-box ATPase, which lacks a residue with comparable property at this position, explaining a discrepant RNA binding mechanism that results in a more flexible binding of the $5^{\prime}$ region (14). The lack of this interaction pattern in Prp43 is likely the reason for the observed decreased binding affinity toward ssRNA (Figure 1C). G-patch proteins interacting with Prp43 have been shown to contact the OBfold domain and thereby stimulate the basal as well as the RNA-dependent ATPase activity of Prp43 $(31,54)$. During this interaction, the G-patch proteins could potentially provide a residue that completes the stacking triad for the missing amino acid, which could lead to a stimulation of the RNA binding and RNA-dependent ATPase activity.

The differences in the binding of the $5^{\prime}$ end of the ssRNA might play a role in the regulation of the translocase/helicase activity of the different members of this family, whereas the vastly conserved interaction pattern of the $3^{\prime}$ end is required to maintain translocation along a single-stranded RNA as a general mechanism.

Intrinsic mobility of RecA2 domain enables open conformation of helicase core

An additional crystal structure of Prp22 in absence of RNA and adenosine nucleotide could be solved (Table 1). The two Prp22 molecules found in the asymmetric unit exhibit virtually identical organization of the RecAl and C-terminal domains, but strongly differ in the position of the RecA2 domain with respect to rest of the protein (Figure 3A). Although significantly elevated $B$-factors of the RecA2 domain did not allow to build a complete model of this domain (133 of 173 RecA2 residues for Apo1 and 141 of 173 RecA2 residues for Apo2), enough portions of the domain were traceable in the electron density to unambiguously place it with respect to the RecA1 and C-terminal domains (Supplementary Figures S3a and S4a). The two different positions of the RecA2 domain together with the increased $B$-factor values suggest that in absence of any interaction partner this domain has a high degree of freedom to move 


\section{Chapter 5: Structural basis for RNA translocation by DEAH-box ATPases}

as a rigid body. In contrast to previously solved adenosine nucleotide-bound structures of genuine DEAH-box ATPases, the RecA2 domains of both Apo structures display an increased distance toward the RecA1 domain (Figure 3B). While the RecA1-RecA2 center of mass distance ranges between 27.3-27.7 $\AA$ when ADP/ATP is bound, this distance is increased to up to $31 \AA$ in the unliganded state $(14,32,37)$. The intrinsic mobility of the RecA2 domain allows the helicase core to adopt more open conformations than were previously known from adenosine nucleotide-bound structures.

The presence of ADP/ATP seems to stabilize closed conformations of the helicase core regardless of the availability of RNA, while in the absence of adenosine nucleotides the bound RNA suppresses the mobility of the RecA2 domain and stabilizes the helicase core in an open conformation with a RecA1-RecA2 center of mass distance of 31.7 $\AA$ (Figure 3B). $B$-factor values of the RecA2 domain are not elevated anymore in the Prp22-RNA complex structure (Supplementary Figure S3b). As a consequence of the open conformation, the adenosine nucleotide-binding site, which is formed by both RecA-like domains does not exhibit a conformation compatible with high-affinity ATP binding.

Prp22 has been found to be in the open conformation in cryo-EM structures of the $\mathrm{C}^{*}$ and post-catalytic $\mathrm{P}$ spliceosome complexes and the Prp22-RNA complex crystal structure fits well into the cryo-EM maps of the $\mathrm{P}$ complexes published by Liu et al. and Wilkinson et al. (Supplementary Figure S5a) $(25,27,58,62,63)$. Spliceosomal DEAH-box ATPases Prp16 and Prp43 acting in the C and intron-lariat spliceosome complexes, respectively, were also modeled with an open conformation of the helicase core $(60,61,64)$. Prp2 represents the only exception, as it has been modeled in the ADP-bound state and thereby closed conformation in two $\mathrm{B}^{\text {act }}$ complex cryo-EM structures $(28,59)$. A fit of the Prp22-RNA complex into the Prp2 cryo-EM map within the $\mathrm{B}^{\text {act }}$ complex published by Yan et al. shows that an open conformation of Prp2 would be in better agreement with the data (Supplementary Figure S5b). A caveat of spliceosomal cryo-EM structures up to date is the significant drop in resolution toward peripheral regions of the complex (65). Since all DEAH-box ATPases are located at the outer rim of the spliceosomes, the resolution for these proteins is low and usually no active-site nucleotide can be identified to accurately assign the catalytic state of the ATPase. However, the information of the adenosine nucleotidespecific domain arrangements known from crystal structures can be used to improve the interpretation of DEAHbox ATPases in spliceosomal cryo-EM structures. The presented adenosine nucleotide-free/open ctPrp22 structures complement the previously known adenosine nucleotidebound/closed DEAH-box ATPase structures and complete the structural repertory of adenosine nucleotide-specific catalytic states.

DEAH-box ATPases translocate at a step-size of one RNA nucleotide per hydrolyzed ATP

Crystallographic studies on DExH-box ATPases over the last decade have revealed that this protein family is able to adopt a variety of conformations and that these structural rearrangements vary as a function of the ligand bound (ATP-bound state, ADP-bound state, adenosine nucleotide-free but RNA-bound state and Apo) $(13,14,23,31,32,37,51,56,57)$. Ligand-specific arrangements of the RecA-like domains are structurally conserved regardless of species and member of the DExH-box family. ADPand ATP-bound structures of Prp43 as well as adenosine nucleotide-free but RNA-bound structures of DHX36 exhibit virtually identical helicase core conformations in different species (Supplementary Figure S6a-c). These conformations are also conserved among different subfamily members since the helicase cores of Prp2, MLE and Prp22 align well with the corresponding structures of Prp43 and DHX36 (Supplementary Figure S6a-c). Combining these structurally conserved catalytic steps, we propose the following model for the single-stranded RNA translocation of DEAH-box ATPases:

In the closed conformation of the helicase core, the adenosine nucleotides are always sandwiched between both RecA-like domains, thereby providing an additional interaction network tightly connecting the two domains $(13,14,23,31,32,37)$. When ATP is bound, this closed conformation allows the conserved sequence motifs Ia, Ib, IV and $\mathrm{V}$ as well as the hook-loop, the hook-turn and the $\beta$ hairpin to interact with the $3^{\prime}$ region of the ssRNA (Supplementary Figure S7a). The transition from ATP to ADP after hydrolysis rearranges the ATP-mediated interaction network bridging RecA1 and RecA2, which induces a reorientation of the domains. The RecA2 domain is rotated by $19^{\circ}$ with a rotation axis close to the center of mass as it is only minimally displaced by $1.8 \AA$ (Supplementary Figure S8b and Supplementary Movie SM1). Up to date, only ADPbound structures in absence of RNA could be solved and no accurate model of an ADP- and RNA-bound DEAHbox ATPase is available. However, by modeling the ssRNA of the ATP-bound structure into the ADP-bound structure via a superposition of the RecA1 domains, the conserved RecA2 residues interacting with the RNA are displaced by $2.6-4.6 \AA$ (Supplementary Figure S7c and d). This displacement results in a loss of contact of the RecA2 domain with the RNA, which is in good agreement with the observed reduced affinity toward RNA in the presence of ADP (Figure 1C). After the release of the ADP, all nucleotide-mediated interactions between both RecAlike domains are lost. Together with the loss of contact of the RecA2 domain with the RNA backbone, this probably enables a complete detachment of the RecA2 domain and allows independent movement as implied by the Prp22 Apo structure (Figure 3A). This positional freedom is suppressed by the interactions of the conserved sequence motifs of the RecA2 domain with the RNA backbone, which shifts this domain by one RNA nucleotide toward the $5^{\prime}$ end (Supplementary Figure S7b). While the DEAH-box ATPase encloses a stack of four RNA nucleotides in the closed and ATP-bound state, this open and adenosine nucleotidefree conformation accommodates a five-nucleotide stack in the RNA-binding tunnel (Figure $4 \mathrm{~A}$ and $\mathrm{B}$ ). During the transition from the closed to the open conformation, the RecA2 $\beta$-hairpin is shifted enough to allow the incorporation of the fifth RNA nucleotide between the preformed four-nucleotide stack and this structural motif (Figure 4C 


\section{Chapter 5: Structural basis for RNA translocation by DEAH-box ATPases}

Nucleic Acids Research, 2019, Vol. 47, No. 84357
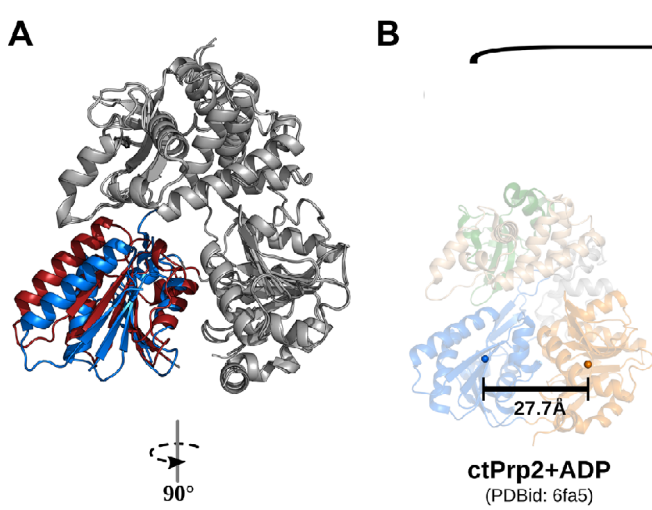

closed conformation

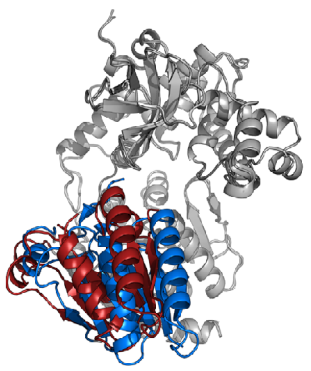

Apo1

Apo2

C

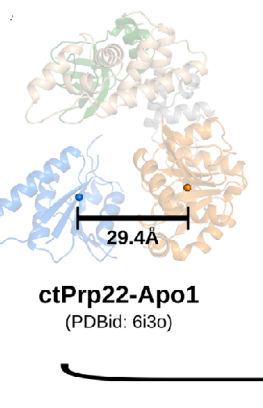

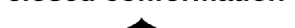

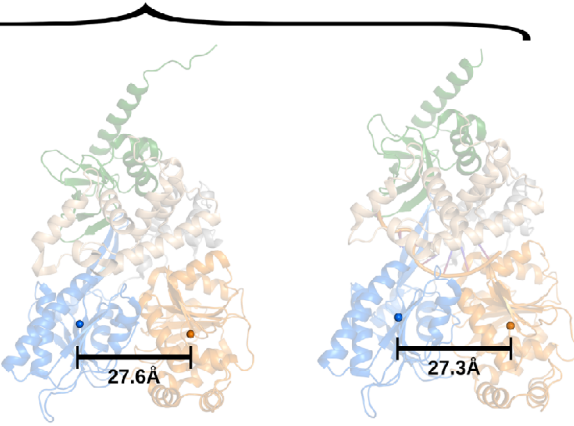

ctPrp43+ADP

(PDBid: 5d0u)

ctPrp43+ADP-BeF
(PDBid: 5 lta)
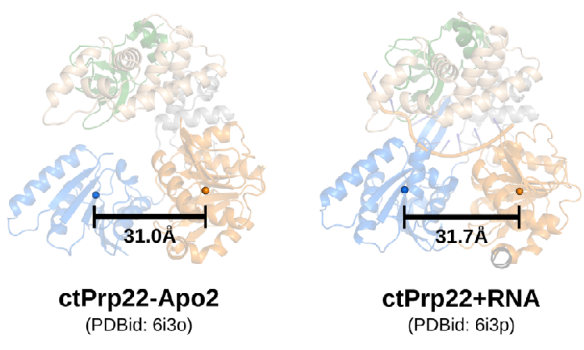

ctPrp22+RNA

(PDBid: 6i3p)

open conformation

Figure 3. Intrinsic mobility of RecA2 domain and comparison of open and closed conformations of the helicase core. (A) Both ctPrp22 molecules found in the asymmetric unit of the Apo structure are depicted as cartoon models. The RecA2 domains are colored in blue (Apo1) and red (Apo2), the RecA1 domains and C-terminal domains are depicted in gray. While the latter domains superpose virtually identically, the RecA2 domains show distinct positions. (B \& C) All structures are depicted as semi-transparent cartoon models and colored according to Figure 1. Center of mass of the RecA-like domains are displayed as spheres and accordingly colored. In order to calculate the centers of mass for the same sets of atoms, the RecA1 and RecA2 domains of the ctPrp22-RNA complex were superimposed with the corresponding domains of the individual catalytic states and centers of mass for these superposed domains were determined. Adenosine nucleotide-bound DEAH-box ATPase structures adopt a closed helicase core conformation (B), whereas ADP/ATPfree structures are found in an open conformation $(\mathbf{C})$.

and Supplementary Movie SM2). In both conformations, the $\beta$-hairpin serves as a physical barrier for the stack and redirects the RNA allowing the $5^{\prime}$ end to interact primarily with the C-terminal domains (Figure 2). Upon ATPbinding to the RNA complex, the RecA2 domain is shifted by $5.8 \AA$, bringing both RecA-like domains back into close proximity (Supplementary Figure S8b). The movement of the RecA2 domain toward the RecA1 domain pushes the ssRNA in $3^{\prime}$ direction through the binding tunnel, spanning a distance equivalent to one RNA nucleotide (Figure $4 C$ ). Due to the higher amount of interactions of the RecA2 domain with the RNA backbone (RecA2: 6 versus RecA1: 3 ) and the mechanical aid of the $\beta$-hairpin, the RNA stays stably attached to the RecA2 domain and does not backlash. Instead, the few RecA1 interactions need to be broken and subsequently replaced by the succeeding sugarphosphates pushed into that position. A cycling of the described events enables DEAH-box ATPases to translocate along an ssRNA in $3^{\prime}-5^{\prime}$ direction at a step-size of one RNA nucleotide per hydrolyzed ATP (Figure 4C; Supplementary Movies SM1 and SM3).

Helicase core rearrangements are driven by a serine in motif $\mathrm{V}$ that senses catalytic state

In order to ensure the right positioning of the RecA2 domain at any catalytic step needed for translocation, DExHbox members require a mechanism to sense the current ligand-bound state. The conserved sequence motif $\mathrm{V}$ has already been suggested to play an important role in the transition between catalytic states, but up to date no detailed molecular mechanism has been proposed for its function $(10,11,13)$. Motif $\mathrm{V}$ is a structural element within the RecA2 domain and it is located in the interface between the adenosine nucleotide-binding site and the RNA-binding tunnel (Figure 5A). At this position it is in close proximity to the bound ADP/ATP and ssRNA. In the Apo, ADPbound and RNA-bound structures this motif forms a short $\alpha$-helix, which is partially distorted when ATP is bound 


\section{Chapter 5: Structural basis for RNA translocation by DEAH-box ATPases}

A

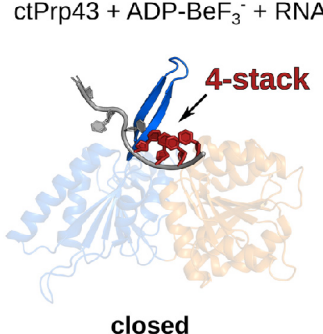

\author{
B
}

ctPrp22 + RNA

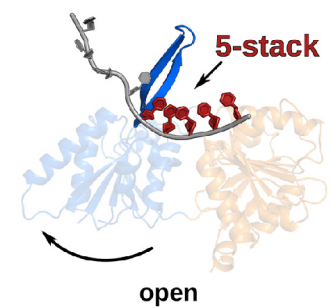

C

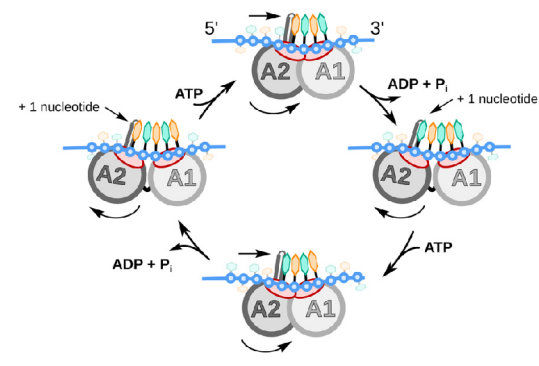

Figure 4. DEAH-box ATPases translocate at a step-size of one RNA nucleotide per hydrolyzed ATP. The helicase cores of ctPrp43 and ctPrp22 with bound ssRNAs are depicted as cartoon models with a domain coloring according to Figure 1. (A) ATP-bound ctPrp43 exhibits a closed conformation of the helicase core with a stack of four RNA nucleotides bound to the RecA-like domains. (B) In the absence of an adenosine nucleotide the helicase core of ctPrp22 adopts an open conformation, which allows the accommodation of an additional RNA nucleotide in the binding tunnel leading to a bound five-nucleotide stack. In both conformations, the stack is interrupted by the $\beta$-hairpin of the RecA2 domain. (C) A continuous cycling of these states 3 to $5^{\prime}$ direction along a single-stranded RNA. Upon helicase core opening, an additional RNA nucleotide can be incorporated between the $\beta$-hairpin and the first RNA nucleotide of the four-nucleotide stack. The helicase core closure induced by the binding of ATP pushes the RNA through the binding tunnel.

(Figure 5B-E; Supplementary Figure S9a and Supplementary Movie SM4). A structural analysis revealed that a serine in motif $\mathrm{V}$ is involved in polar interactions with one of the ligands in all structurally characterized catalytic states. In the adenosine nucleotide-free but RNA-bound state, the side chain of the serine contacts the $\mathrm{U}_{7}$ phosphate and thereby bridges the gap that is formed by the more distant conformation of the RecA-like domains (Figure 5C). In the ADP/ATP-bound structures, the mainchain of this residue interacts via hydrogen-bonds with conserved water molecules constituting the active site (Figure 5D and E). The side chain of the serine additionally interacts with another water molecule and two active site residues when ATP is present (Figure 5E). The interactions in the ATP-bound state force the serine mainchain to adopt an unfavored conformation, enabling the mainchain nitrogen to hydrogen bond with the catalytic water (Supplementary Figure S9d). As a consequence, the $\mathrm{C}$-terminus of the motif $\mathrm{V}$ helix is disrupted (Figure 5B). Upon ATP hydrolysis, the interactions at the active site are rearranged and the serine looses its hydrogen bond network allowing the serine mainchain and the entire motif $\mathrm{V}$ to relax into the energetically more favorable helical conformation. This contributes to a global repositioning of the RecA2 domain, since the helical motif V conformation would clash with motif Ia of the RecA1 domain in the ATP-bound helicase core arrangement (Figure 5F and Supplementary Figure S9e). This steric hindrance forces the RecA2 domain to interact with RNA nucleotides positioned one residue further to the $5^{\prime}$ end when no adenosine nucleotide is present and thereby achieving the one RNA nucleotide step-size. Such a mechanism shows parallels to the loaded-spring model of $\mathrm{G}$ domain proteins and ATP-dependent motor proteins like kinesin and myosin (66-68). Here, the so-called conserved switch regions act as $\gamma$-phosphate sensors that are released in a spring-like manner upon NTP hydrolysis, triggering conformational changes of other domains (69).

In order to verify the central role of this serine, we created alanine, glycine and proline mutants of this residue in Prp22 and characterized their ATPase activity (Figure 5G). The lack of the hydroxyl group in Prp22-S837A mainly leads to an increase of $k_{\text {cat }}$ regardless of the presence of RNA and only to a minor decrease of $K_{\mathrm{m}}$ when RNA is present. In both cases, the catalytic efficiency $\left(k_{\mathrm{cat}} / K_{\mathrm{m}}\right)$ is improved by a factor of $\sim 1.7$. Since mainly the mainchain of the motif $\mathrm{V}$ serine interacts via water-mediated hydrogen bonds with the adenosine nucleotides, we tested if a higher flexibility of the mainchain at this position has an effect on catalysis. The substitution by a glycine in Prp22-S837G has no significant effect on $k_{\text {cat }}$ compared to the alanine mutant, but significantly improves the $K_{\mathrm{m}}$, especially in the presence of RNA. The catalytic efficiency increases 2.3 -fold in the absence of RNA and 3.7-fold in the presence of RNA. The gain in flexibility at this position in motif $\mathrm{V}$ appears to improve the binding/sensing of ATP rather than stimulating the turnover rate. In contrast to an increase in flexibility of the mainchain at this position, we tested a decrease in flexibility by mutating the serine to a proline. Prp22-S837P did not show any significant ATPase activity (data not shown). In order to exclude misfolding as a reason for the lack in activity, we subjected Prp22 and Prp22-S837P to circular dichroism spectroscopy measurements and could verify the structural integrity of Prp22-S837P (Supplementary Figure S9f). We could furthermore structurally characterize Prp22S837A in the adenosine nucleotide-free but RNA-bound state, as well as Prp43-S387A and Prp43-S387G in the ATPbound state (Table 1). Since Prp22 could not be crystallized in the presence of an ATP-analog, an existing crystallization condition for ADP- $\mathrm{BeF}_{3}{ }^{-}$-bound $\operatorname{Prp} 43$ was used to structurally characterize motif $\mathrm{V}$ serine mutants of a DEAH-box ATPase in this state (14). The structures of these three mutants show that motif $\mathrm{V}$ is able to adopt the same nucleotidespecific conformations regardless of the side chain property (Supplementary Figure S9b and c).

Summarizing our data show that the serine side chain is dispensable for the function of motif $\mathrm{V}$ and instead has a regulatory role. The polar interactions of the hydroxyl group with the RNA phosphate and active site components seem to negatively regulate the turnover rate of DEAH-box ATPases. Instead, the mainchain is sufficient for sensing the adenosine nucleotide state at the active site and this sensing function is sensitive to the flexibility of the mainchain. 
A
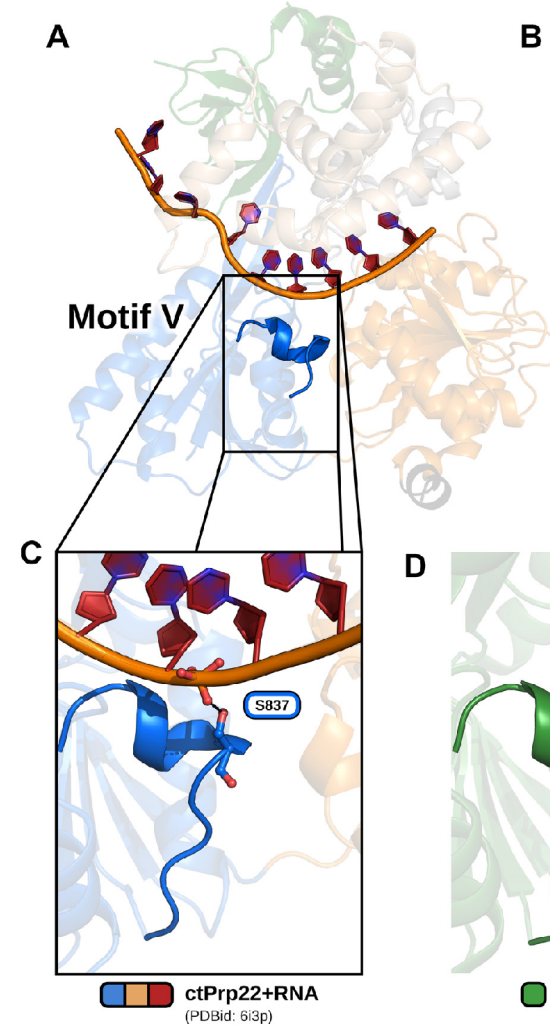

$\mathbf{F}$

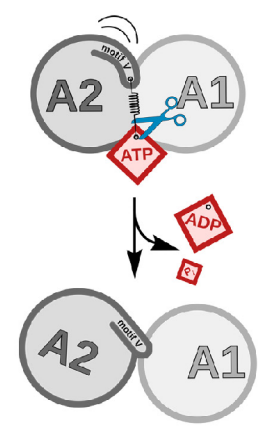

B

D

G
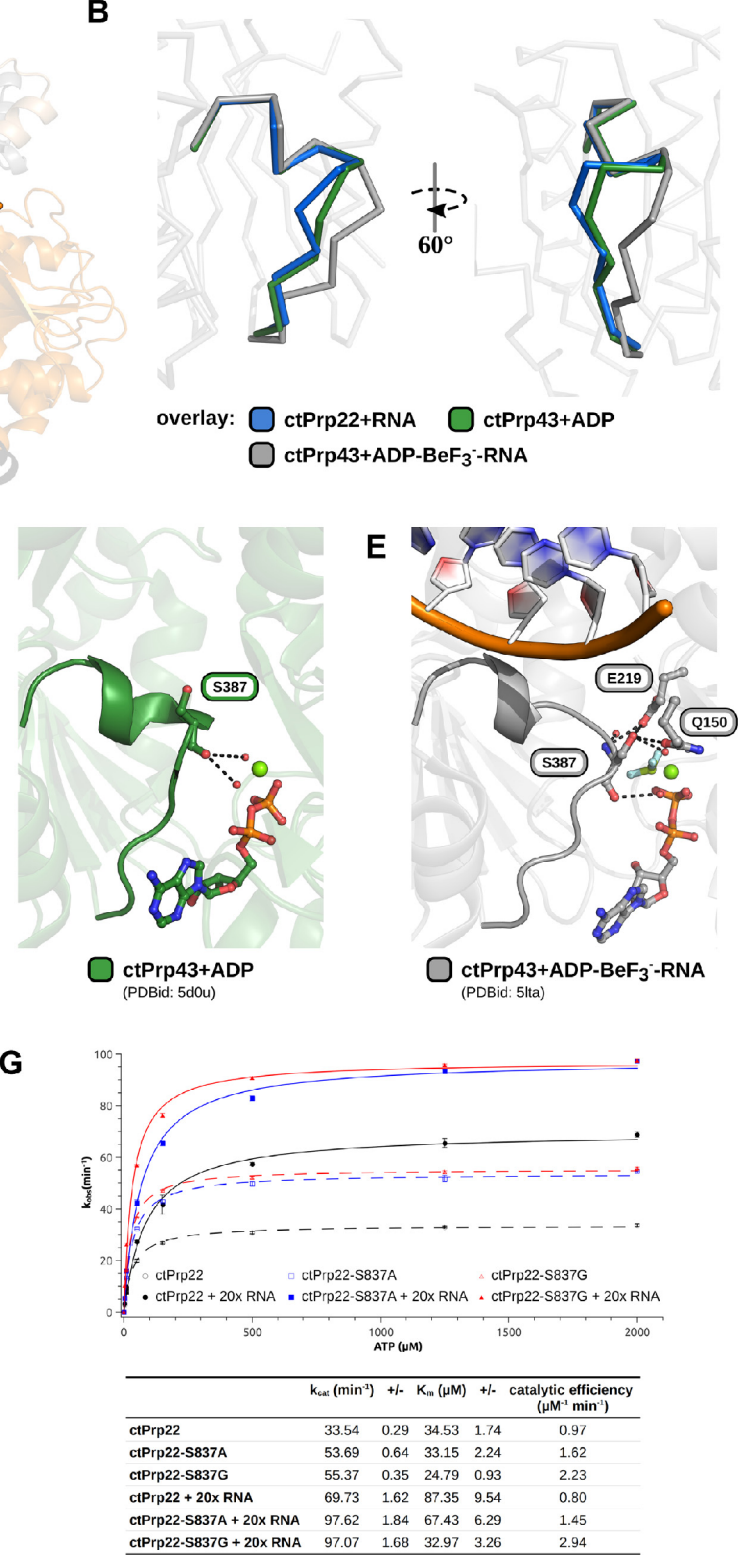

Figure 5. A serine in motif $\mathrm{V}$ senses the catalytic state. (A) Motif $\mathrm{V}$ is located in the RecA2 domain in close proximity to the RNA-binding tunnel and the active site. (B) Motif $\mathrm{V}$ exhibits a helical conformation in the absence of adenosine nucleotides, as well as when ADP is bound. In the presence of ATP, the helix is disrupted and the motif adopts an alternative conformation. A serine in this motif senses the current catalytic state via different polar interactions with the bound ligands (C-E). In the adenosine nucleotide-free but RNA-bound state, the serine side chain hydrogen bonds with an ssR NA phosphate $(C)$, and in the presence of ADP, the mainchain interacts with two water molecules coordinated by the active site magnesium (D). (E) When ATP is bound, the motif $\mathrm{V}$ helix is distorted by the interactions of the serine with numerous active site components. The mainchain interacts with the catalytic water molecule and a magnesium-coordinated water molecule. The side chain undergoes polar interactions with an active site glutamate and glutamine as well as with another water molecule coordinated by the magnesium. (F) The ATP-dependent interactions of the serine force motif V into an unfavored conformation. Upon ATP hydrolysis the motif is able to relax back into its helical conformation, which is translated into a repositioning of the RecA2 domain. (G) ATPase activity measurements of ctPrp22-S837A and ctPrp22-S837G mutants compared with wild-type ctPrp22 in absence and in presence of $\mathrm{A}_{20}$-ssRNA. The experiments were determined in triplicates and error bars for each measured data point are depicted. The error of fit is indicated in the table as $+/-$. 


\section{Chapter 5: Structural basis for RNA translocation by DEAH-box ATPases}

4360 Nucleic Acids Research, 2019, Vol. 47, No. 8

While the ATP binding is improved at maximum flexibility (glycine), the sensing function is completely abolished at minimum flexibility (proline).

\section{CONCLUSIONS}

In eukaryotes non-coding introns in precursor mRNAs are excised via the spliceosome. One key player during premRNA splicing is the DEAH-box ATPase Prp22, which is critical for the release of mature mRNAs. In this study, we determined the first crystal structures of the spliceosomal DEAH-box ATPase Prp22 in the adenosine nucleotide-free but RNA-bound state, as well as in absence of any ligand. Prp22 shares a conserved architecture of the catalytic unit with other spliceosomal DEAH-box ATPases and interacts with RNA in a comparable manner as Prp43. In the absence of RNA and adenosine nucleotide, the RecA2 domain has an increased ability to move independently as a rigid body and a serine in motif $\mathrm{V}$ ensures the proper positioning of this domain in dependence of the bound ligand state. A comparison with the ATP-bound state of Prp43 shows that these ATPases toggle between a closed conformation of the helicase core, which accommodates a stack of four nucleotides in the RNA-binding tunnel, and an open conformation with a bound five-nucleotide stack. A cycling of these events enables these proteins to translocate in $3^{\prime}-5^{\prime}$ direction along a single-stranded RNA with a step-size of one RNA nucleotide per hydrolyzed ATP (Supplementary Movie SM1 and SM3). The proposed model might also provide an explanation for the RNA-dependent ATPase stimulation as a common feature of DEAH-box ATPases. In the RNA bound but adenosine nucleotide-free structure, the RecA2 domain is fixed by the RNA in a conformation distant enough from the RecAl domain to provide good accessibility for the binding of ATP, but close enough to directly sense the bound ATP by the motif $\mathrm{V}$ serine and induce helicase core closure. In contrast, without bound RNA the RecA2 domain is not optimally prepositioned due to its intrinsic mobility, leading to a prolonged ATP sensing time.

Two different models for the translocation of DEAH-box ATPases have been proposed in the past $(13,14,51)$. The contraction model suggests a continuous transition between a three- and a four-nucleotide stack in the RNA-binding tunnel, caused by primarily smaller rotational movements of the helicase core (13). However, the open helicase core conformation of Prp22 with a stack of five RNA nucleotides strongly argues in favor of the expansion model (13). This model can most likely be extended to other genuine DEAHbox members as they are all highly conserved in sequence and structure. In fact, this translocation mechanism seems to be applicable to the entire DExH-box family, since the recently solved structure of nucleotide-free but nucleic acidbound DHX36 shows a similar open conformation as the ctPrp22+RNA complex (Supplementary Figures S6c and S8a) $(56,57)$. DExH-box members, DHX36 and MLE, even harbor an almost identical motif $\mathrm{V}$ to drive the opening and closing of the helicase core.

Even for more distant relatives of the SF2 helicase family this translocation mechanism seems to be conserved.
Using structural and biophysical approaches like smFRET a translocation step-size of as well one RNA nucleotide could be determined for NS3 helicases (70-72). These helicases also translocate along an ssRNA by repeatedly cycling between open and closed conformations of the helicase core upon continuous ATP hydrolysis (Supplementary Figure S10a and b). While the architecture of the helicase core seems to be highly conserved between DExH-box and NS3 members, the C-terminal domains differ significantly (Supplementary Figure S11). Due to the much smaller Cterminal domain, NS3 helicases possess a shorter RNAbinding tunnel that only accommodates the stacked RNA region (Supplementary Figure S10). NS3 helicases thus lack an extensive interaction network with the C-terminal domain as seen for the $5^{\prime}$ end of the ssRNA in complex with Prp22. This independence from a C-terminal domain completely decouples the translocation mechanism primarily driven by the RecA-like domains from any potential regulatory function this additional $5^{\prime}$-interaction might have in DEAH-box ATPases. It also leads to a different trajectory of the RNA through the tunnel accompanied by a divergent position of the RecA2 domain, especially in the ATPbound state (Supplementary Figure S10c and d). While the adenosine nucleotide-specific helicase core arrangement slightly differs among these two SF2 members, the mechanism to sense the nucleotide state and accordingly position the RecA2 domain is likely conserved. Despite the difference in sequence conservation, a structurally conserved motif $\mathrm{V}$ is also present in NS3 helicases (Supplementary Figure $\mathrm{S} 12 \mathrm{a}-\mathrm{c})$. The proposed motif $\mathrm{V}$ sensing mechanism also applies to NS3 helicases as this motif also switches from a helical toward a distorted conformation in an adenosine nucleotide-dependent manner (Supplementary Figure S12a and b). Interestingly, the sensor serine of DExH-box ATPases is replaced by a glycine in NS3 helicases and more distant relatives like the Ski2-like helicases and even in members of the SF1 family (Supplementary Figure S12c). The lack of a side chain at this residue position in motif $\mathrm{V}$ of other SF2 members is in agreement with the findings that it is dispensable for the function. The increased flexibility of a glycine at this position in other SF2 members might be required to ensure the sensing mechanism due to the different helicase core conformations. Apart from the described structural differences, NS3 helicases seem to show also some mechanistic differences. While in NS3 helicases the ATP-bound state is defined as the low-affinity state and the adenosine nucleotide-free state as the high-affinity state towards ssRNA, DEAH-box ATPases exhibit rather the opposite (Figure 1C). Summarizing, while the motif V driven helicase core rearrangements of DExH-box ATPases and NS3 helicases show parallels in agreement with the expansion model for translocation, differences in size and property of the C-terminal domains might have a regulatory impact causing structural and mechanistic variations among these SF2 members. It also remains unclear if the expansion mechanism shared by DExH-box and NS3 members is more universally conserved and could apply as well to other members of the SF2 family known to depend on translocation, like the Ski2-like helicases (12). 


\section{Chapter 5: Structural basis for RNA translocation by DEAH-box ATPases}

\section{DATA AVAILABILITY}

The coordinates and structure factors have been deposited in the PDB, with following accession numbers:

- $6 \mathrm{I} 3 \mathrm{O}$ - ctPrp22 Apo

- 6I3P - ctPrp22 + RNA

- 6QIC - ctPrp22-S837A + RNA

- 6QID - ctPrp43-S387A + ADP-BeF ${ }_{3}^{-}$

- 6QIE - ctPrp43-S387G + ADP-BeF 3 -

\section{SUPPLEMENTARY DATA}

Supplementary Data are available at NAR Online.

\section{ACKNOWLEDGEMENTS}

We acknowledge DESY (Hamburg, Germany), a member of the Helmholtz Association HGF, for the provision of experimental facilities. Parts of this research were carried out at PETRA III and we would like to thank Saravanan Panneerselvam and Johannna Hakanpää for assistance in using EMBL beamlines P13 and P14. Furthermore, we are thankful to Dr Piotr Neumann for critical reading of the manuscript and crystallographic advice. We are also grateful to Kelly Libnik for the outstanding assistance in protein production and to Dr Viktor Sautner (Department of Molecular Enzymology, University of Göttingen) for the support concerning the circular dichroism measurements. Author contributions: Experimental design (F.H., R.F.), molecular cloning (F.H., M.E.), protein expression and purification (F.H., M.E.), crystallization of protein complexes (F.H., M.E.), data collection and processing (F.H., M.E.), structure determination and refinement (F.H., M.E.), crystal structure analysis (F.H., R.F.), ATPase activity assays (F.H.), RNA-binding assays (F.H.), circular dichroism spectroscopy (F.H., M.E.), data analysis (F.H., R.F.), preparation of figures/tables/movies (F.H.), writing - original draft (F.H.), writing - review and editing (F.H., R.F.), supervision, project leader (R.F.).

\section{FUNDING}

Deutsche Forschungsgemeinschaft (DFG) [SFB 860 TPA02, INST186/1117]. We acknowledge support by the German Research Foundation and the Open Access Publication Funds of the Goettingen University. Conflict of interest statement. None declared.

\section{REFERENCES}

1. Will,C.L. and Lührmann,R. (2011) Spliceosome structure and function. Cold Spring Harb. Perspect. Biol., 3, a003707.

2. Hoskins,A.A. and Moore,M.J. (2012) The spliceosome: a flexible, reversible macromolecular machine. Trends Biochem. Sci., 37, $179-188$.

3. Matera,A.G. and Wang,Z. (2014) A day in the life of the spliceosome. Nat. Rev. Mol. Cell Biol., 15, 108-121.

4. Cordin,O. and Beggs,J.D. (2013) RNA helicases in splicing. RNA Biol., 10, 83-95.

5. Ding,S.C. and Pyle,A.M. (2012) Molecular mechanics of RNA translocases. Meth. Enzymol., 511, 131-147.

6. Ozgur,S., Buchwald,G., Falk,S., Chakrabarti,S., Prabu,J.R. and Conti,E. (2015) The conformational plasticity of eukaryotic RNA-dependent ATPases. FEBS J., 282, 850-863.
7. Fairman-Williams,M.E., Guenther,U.-P. and Jankowsky,E. (2010) SF1 and SF2 helicases: family matters. Curr. Opin. Struct. Biol., 20, 313-324

8. Schwer,B. and Meszaros,T. (2000) RNA helicase dynamics in pre-mRNA splicing. EMBO J., 19, 6582-6591.

9. Campodonico,E. and Schwer,B. (2002) ATP-dependent remodeling of the spliceosome: intragenic suppressors of release-defective mutants of Saccharomyces cerevisiae Prp22. Genetics, 160, 407-415.

10. Schneider,S., Campodonico,E. and Schwer,B. (2004) Motifs IV and $\mathrm{V}$ in the DEAH box splicing factor Prp22 are important for RNA unwinding, and helicase-defective Prp22 mutants are suppressed by Prp8. J. Biol. Chem., 279, 8617-8626.

11. Tanaka,N. and Schwer,B. (2006) Mutations in PRP43 that uncouple RNA-dependent NTPase activity and pre-mRNA splicing function. Biochemistry, 45, 6510-6521.

12. Büttner,K., Nehring,S. and Hopfner,K.-P. (2007) Structural basis for DNA duplex separation by a superfamily-2 helicase. Nat. Struct. Mol. Biol., 14, 647-652.

13. He,Y., Staley,J.P., Andersen,G.R. and Nielsen,K.H. (2017) Structure of the DEAH/RHA ATPase Prp43p bound to RNA implicates a pair of hairpins and motif $\mathrm{Va}$ in translocation along RNA. RNA, 23, $1110-1124$.

14. Tauchert,M.J., Fourmann,J.-B., Lührmann,R. and Ficner,R. (2017) Structural insights into the mechanism of the DEAH-box RNA helicase Prp43. eLife, 6, e21510.

15. Schwer,B. (2008) A conformational rearrangement in the spliceosome sets the stage for Prp22-dependent mRNA release. Mol. Cell, 30, 743-754.

16. Company,M., Arenas,J. and Abelson,J. (1991) Requirement of the RNA helicase-like protein PRP22 for release of messenger RNA from spliceosomes. Nature, 349, 487-493.

17. Fabrizio,P., Dannenberg,J., Dube,P., Kastner,B., Stark,H., Urlaub,H. and Lührmann,R. (2009) The evolutionarily conserved core design of the catalytic activation step of the yeast spliceosome. Mol. Cell, 36, 593-608.

18. Mayas,R.M., Maita,H. and Staley,J.P. (2006) Exon ligation is proofread by the DExD/H-box ATPase Prp22p. Nat. Struct. Mol. Biol., 13, 482-490.

19. Semlow,D.R., Blanco,M.R., Walter,N.G. and Staley,J.P. (2016) Spliceosomal DEAH-Box ATPases remodel Pre-mRNA to activate alternative splice sites. Cell, 164, 985-998.

20. Tanaka,N. and Schwer,B. (2005) Characterization of the NTPase, RNA-binding, and RNA helicase activities of the DEAH-Box splicing factor Prp22. Biochemistry, 44, 9795-9803.

21. Wagner,J.D.O., Jankowsky,E., Company,M., Pyle,A.M. and Abelson,J.N. (1998) The DEAH-box protein PRP22 is an ATPase that mediates ATP-dependent mRNA release from the spliceosome and unwinds RNA duplexes. EMBO J., 17, 2926-2937.

22. Tanaka,N., Aronova,A. and Schwer,B. (2007) Ntr1 activates the Prp43 helicase to trigger release of lariat-intron from the spliceosome. Genes Dev., 21, 2312-2325.

23. He,Y., Andersen,G.R. and Nielsen,K.H. (2010) Structural basis for the function of DEAH helicases. EMBO Rep., 11, 180-186.

24. Wang,Y and Guthrie,C. (1998) PRP16, a DEAH-box RNA helicase, is recruited to the spliceosome primarily via its nonconserved $\mathrm{N}$-terminal domain. $R N A, \mathbf{4}, 1216-1229$

25. Liu,S., Li,X., Zhang,L., Jiang,J., Hill,R.C., Cui,Y., Hansen,K.C., Zhou,Z.H. and Zhao,R. (2017) Structure of the yeast spliceosomal postcatalytic P complex. Science, 358, 1278-1283.

26. Wan,R., Yan,C., Bai,R., Lei,J. and Shi,Y. (2017) Structure of an intron lariat spliceosome from Saccharomyces cerevisiae. Cell, 171, $120-132$.

27. Fica,S.M., Oubridge,C., Galej,W.P., Wilkinson,M.E., Bai,X.-C., Newman,A.J. and Nagai,K. (2017) Structure of a spliceosome remodelled for exon ligation. Nature, 542, 377-380.

28. Yan,C., Wan,R., Bai,R., Huang,G. and Shi,Y. (2016) Structure of a yeast activated spliceosome at $3.5 \AA$ resolution. Science, 353, 904-911.

29. Schwer,B. and Guthrie,C. (1991) PRP16 is an RNA-dependent ATPase that interacts transiently with the spliceosome. Nature, $\mathbf{3 4 9}$ 494499.

30. Schwer,B. and Gross,C.H. (1998) Prp22, a DExH-box RNA helicase, plays two distinct roles in yeast pre-mRNA splicing. EMBO J., 17, 2086-2094. 


\section{Chapter 5: Structural basis for RNA translocation by DEAH-box ATPases}

31. Walbott,H., Mouffok,S., Capeyrou,R., Lebaron,S., Humbert,O., van Tilbeurgh,H., Henry, Y, and Leulliot,N. (2010) Prp43p contains a processive helicase structural architecture with a specific regulatory domain $E M B O J, 29,2194-2204$

32. Tauchert,M.J., Fourmann,J.-B., Christian,H., Lührmann,R. and Ficner,R. (2016) Structural and functional analysis of the RNA helicase Prp43 from the thermophilic eukaryote Chaetomium thermophilum. Acta Crystallogr. F Struct. Biol. Commun., 72 $112-120$.

33. Altschul,S.F., Gish,W., Miller,W., Myers,E.W. and Lipman,D.J. (1990) Basic local alignment search tool. J. Mol. Biol., 215, 403-410.

34. Studier,F.W. (2014) Stable expression clones and auto-induction for protein production in E. coli. Methods Mol. Biol., 1091, 17-32.

35. Kabsch,W. (2010) XDS. Acta Crystallogr. D Biol. Crystallogr., 66, $125-132$

36. McCoy,A.J., Grosse-Kunstleve,R.W., Adams,P.D., Winn,M.D., Storoni,L.C. and Read,R.J. (2007) Phaser crystallographic software. J. Appl. Crystallogr., 40, 658-674.

37. Schmitt,A., Hamann,F., Neumann,P. and Ficner,R. (2018) Crystal structure of the spliceosomal DEAH-box ATPase Prp2. Acta Crystallogr. D Struct. Biol., 74, 643-654.

38. Stein,N. (2008) CHAINSAW: a program for mutating pdb files used as templates in molecular replacement. J. Appl. Cryst., 41, 641-643.

39. Emsley,P., Lohkamp, B., Scott,W.G. and Cowtan, K. (2010) Features and development of Coot. Acta Crystallogr. D Biol. Crystallogr., 66, 486-501.

40. Murshudov, G.N., Skubák,P., Lebedev,A.A., Pannu,N.S., Steiner,R.A., Nicholls,R.A., Winn,M.D., Long,F. and Vagin,A.A. (2011) REFMAC5 for the refinement of macromolecular crystal structures. Acta Crystallogr. D Biol. Crystallogr., 67, 355-367.

41. Winn,M.D., Isupov,M.N. and Murshudov, G.N. (2001) Use of TLS parameters to model anisotropic displacements in macromolecular refinement. Acta Crystallogr. D Biol. Crystallogr., 57, 122-133.

42. Nicholls, R.A., Long,F. and Murshudov,G.N. (2012) Low-resolution refinement tools in REFMAC5. Acta Crystallogr. D Biol. Crystallogr., 68, 404-417.

43. Chen,V.B., Arendall,W.B., Headd,J.J., Keedy,D.A., Immormino,R.M., Kapral,G.J., Murray,L.W., Richardson,J.S. and Richardson,D.C. (2010) MolProbity: all-atom structure validation for macromolecular crystallography. Acta Crystallogr. D Biol. Crystallogr, 66, 12-21.

44. Kleywegt,G.J. (1996) Use of non-crystallographic symmetry in protein structure refinement. Acta Crystallogr D Biol Crystallogr., 52, 842-857

45. Pettersen,E.F., Goddard,T.D., Huang,C.C., Couch,G.S., Greenblatt,D.M., Meng,E.C. and Ferrin,T.E. (2004) UCSF Chimera-a visualization system for exploratory research and analysis. J. Comput. Chem., 25, 1605-1612.

46. Agarwal,K.C., Miech,R.P. and Parks,R.E. (1978) Guanylate kinases from human erythrocytes, hog brain, and rat liver. Meth. Enzymol., 51, 483-490.

47. Rossi,A.M. and Taylor,C.W. (2011) Analysis of protein-ligand interactions by fluorescence polarization. Nat. Protoc., 6, 365-387.

48. Kelly,S.M., Jess,T.J. and Price,N.C. (2005) How to study proteins by circular dichroism. Biochim. Biophys. Acta, 1751, 119-139.

49. Amlacher,S., Sarges,P., Flemming,D., van Noort,V., Kunze, R. Devos,D.P., Arumugam,M., Bork,P. and Hurt,E. (2011) Insight into structure and assembly of the nuclear pore complex by utilizing the genome of a eukaryotic thermophile. Cell, 146, 277-289.

50. Schneider,S. and Schwer,B. (2001) Functional domains of the yeast splicing factor Prp22p. J. Biol. Chem., 276, 21184-21191.

51. Prabu,J.R., Müller,M., Thomae,A.W., Schüssler,S., Bonneau,F., Becker,P.B. and Conti,E. (2015) Structure of the RNA helicase MLE reveals the molecular mechanisms for uridine specificity and RNA-ATP coupling. Mol. Cell, 60, 487-499.

52. Kim,S.H., Smith,J., Claude,A. and Lin,R.J. (1992) The purified yeast pre-mRNA splicing factor PRP2 is an RNA-dependent NTPase. EMBO J., 11, 2319-2326.
53. Warkocki,Z., Schneider,C., Mozaffari-Jovin,S., Schmitzová,J., Höbartner, C., Fabrizio,P. and Lührmann, R. (2015) The G-patch protein Spp2 couples the spliceosome-stimulated ATPase activity of the DEAH-box protein Prp2 to catalytic activation of the spliceosome. Genes Dev., 29, 94-107.

54. Christian,H., Hofele,R.V., Urlaub,H. and Ficner,R. (2014) Insights into the activation of the helicase Prp43 by biochemical studies and structural mass spectrometry. Nucleic Acids Res. 42, 1162-1179.

55. Heininger,A.U., Hackert,P. Andreou,A.Z., Boon,K.-L., Memet,I. Prior,M., Clancy,A., Schmidt,B., Urlaub,H., Schleiff,E. et al. (2016) Protein cofactor competition regulates the action of a multifunctional RNA helicase in different pathways. RNA Biol., 13, 320-330.

56. Chen,M.C., Tippana,R., Demeshkina,N.A., Murat,P., Balasubramanian,S., Myong,S. and Ferré-D’Amaré,A.R. (2018) Structural basis of G-quadruplex unfolding by the DEAH/RHA helicase DHX36. Nature, 558, 465-469.

57. Chen,W.-F., Rety,S., Guo,H.-L., Dai,Y.-X., Wu,W.-Q., Liu,N.-N., Auguin,D., Liu,Q.-W., Hou,X.-M., Dou,S.-X. et al. (2018) Molecular mechanistic insights into Drosophila DHX36-mediated G-Quadruplex unfolding: a structure-based model. Structure, 26 , $403-415$.

58. Wilkinson,M.E., Fica,S.M., Galej,W.P., Norman,C.M., Newman,A.J. and Nagai,K. (2017) Postcatalytic spliceosome structure reveals mechanism of $3^{\prime}$-splice site selection. Science, 358, 1283-1288.

59. Rauhut,R., Fabrizio,P., Dybkov,O., Hartmuth,K., Pena,V., Chari,A., Kumar,V., Lee,C.-T., Urlaub,H., Kastner,B. et al. (2016) Molecular architecture of the Saccharomyces cerevisiae activated spliceosome. Science, 353, 1399-1405.

60. Wan,R., Yan,C., Bai,R., Lei,J. and Shi,Y. (2017) Structure of an intron lariat spliceosome from Saccharomyces cerevisiae. Cell, 171, $120-132$.

61. Galej,W.P., Wilkinson,M.E., Fica,S.M., Oubridge,C., Newman,A.J. and Nagai,K. (2016) Cryo-EM structure of the spliceosome immediately after branching. Nature, 537, 197-201.

62. Yan,C., Wan, R., Bai,R., Huang,G. and Shi,Y. (2017) Structure of a yeast step II catalytically activated spliceosome. Science, $\mathbf{3 5 5}$, 149-155.

63. Bai,R., Yan,C., Wan,R., Lei,J. and Shi,Y. (2017) Structure of the post-catalytic spliceosome from Saccharomyces cerevisiae. Cell, 171, 1589-1598.

64. Wan,R., Yan,C., Bai,R., Huang,G. and Shi,Y. (2016) Structure of a yeast catalytic step I spliceosome at $3.4 \AA$ resolution. Science, $\mathbf{3 5 3}$, 895-904.

65. Ficner,R., Dickmanns,A. and Neumann,P. (2017) Studying structure and function of spliceosomal helicases. Methods, 125, 63-69.

66. Vetter,I.R. and Wittinghofer,A. (2001) The guanine nucleotide-binding switch in three dimensions. Science, 294 1299-1304.

67. Milburn,M.V., Tong,L., deVos,A.M., Brünger,A., Yamaizumi,Z., Nishimura,S. and Kim,S.H. (1990) Molecular switch for signal transduction: structural differences between active and inactive forms of protooncogenic ras proteins. Science, 247, 939-945.

68. Kull,F.J. and Endow,S.A. (2002) Kinesin: switch I \& II and the motor mechanism. J. Cell. Sci., 115, 15-23.

69. Roll-Mecak,A., Cao,C., Dever,T.E. and Burley,S.K. (2000) X-Ray structures of the universal translation initiation factor IF2/eIF5B conformational changes on GDP and GTP binding. Cell, 103, 781-792.

70. Myong,S., Bruno,M.M., Pyle,A.M. and Ha,T. (2007) Spring-loaded mechanism of DNA unwinding by hepatitis C virus NS3 helicase. Science, 317, 513-516.

71. Gu,M. and Rice,C.M. (2010) Three conformational snapshots of the hepatitis $C$ virus NS3 helicase reveal a ratchet translocation mechanism. Proc. Natl. Acad. Sci. U.S.A., 107, 521-528.

72. Appleby,T.C., Anderson,R., Fedorova,O., Pyle,A.M., Wang,R., Liu,X., Brendza,K.M. and Somoza,J.R. (2011) Visualizing ATP-dependent RNA translocation by the NS3 helicase from $\mathrm{HCV}$. J. Mol. Biol., 405, 1139-1153. 
Chapter 5: Structural basis for RNA translocation by DEAH-box ATPases

\section{Supplementary data}

a

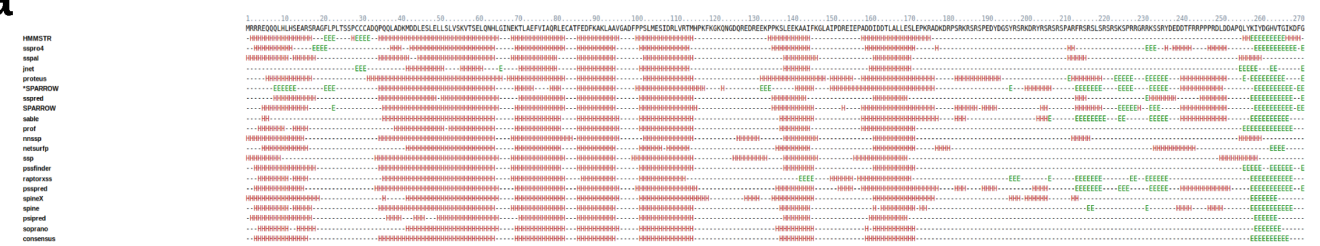

b

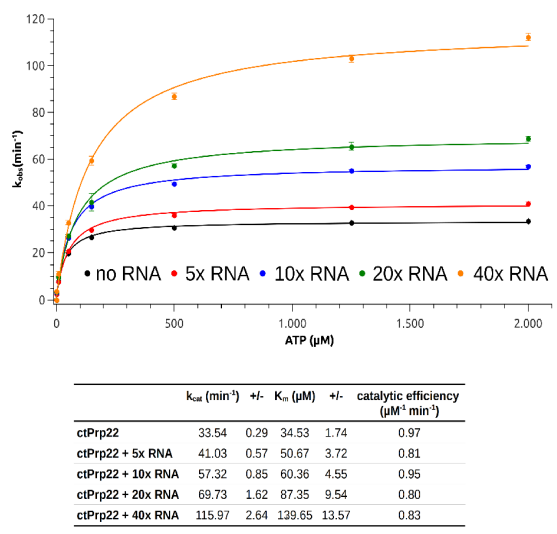

C

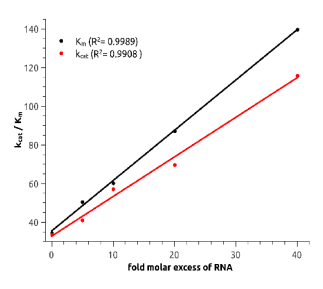

Supplemental figure S1

Secondary structure prediction of the N-terminus of Chaetomium thermophilum Prp22 and analysis of RNA-dependent stimulation of ATPase activity of ctPrp22. (a) The GeneSilico metaserver was used to predict the secondary structure of the N-terminal extension of ctPrp22 (Kurowski and Bujnicki 2003 ). Individual prediction server are stated first, followed by the corresponding prediction to the right. Residues predicted as part of $\alpha$-helices are highlighted by red " $\mathrm{H}$ " and residues predicted as part of $\beta$-strands are displayed as green "E". (b) To examine the RNA-dependent stimulation of ctPrp22, the ATPase activity was measured at different RNA concentrations up to 40 -fold molar excess. $k_{\text {cat }}$ and $K_{\mathrm{m}}$ are stimulated in a linear fashion by increasing concentrations of RNA. At 40 -fold molar excess ctPrp22 exhibits a 3.5-fold stimulation compared to the ATPase activity in absence of RNA. 


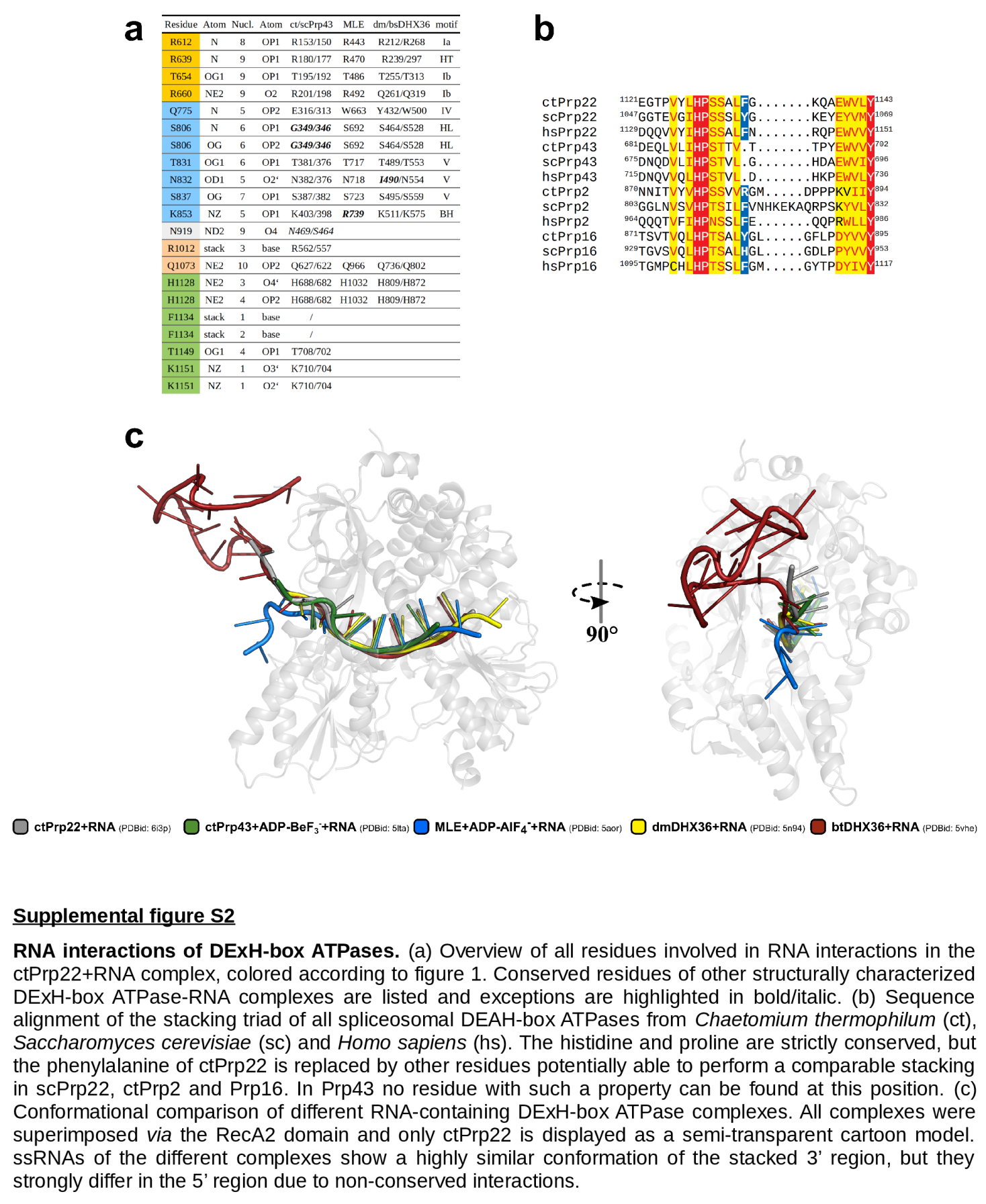



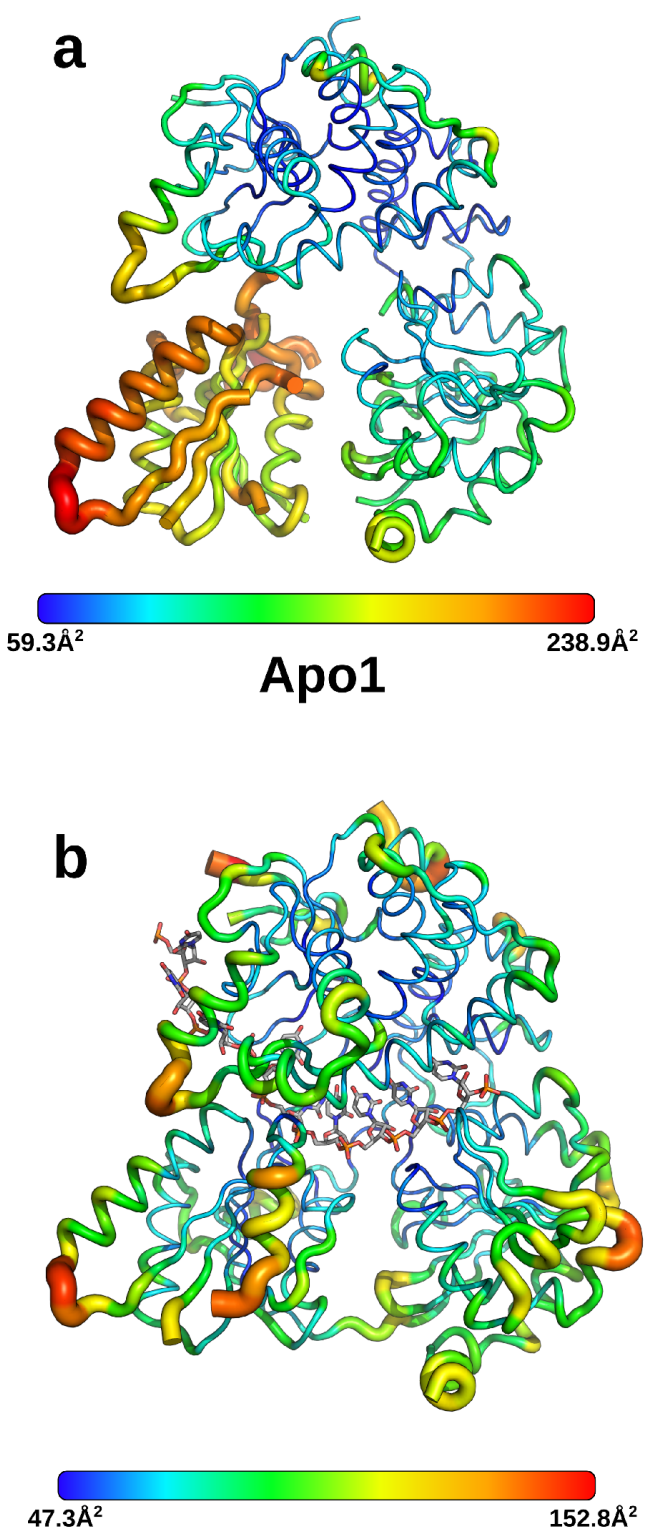

d

\begin{tabular}{lcc}
\hline & ctPrp22 Apo & $\begin{array}{l}\text { ctPrp22 + } \\
\text { RNA }\end{array}$ \\
\hline A - B & $2.63^{\star}\left(570^{\star \star}\right)$ & $0.51(621)$ \\
A - C & $/$ & $0.41(625)$ \\
A - D & $/$ & $0.39(626)$ \\
B - C & $/$ & $0.42(623)$ \\
B - D & $/$ & $0.50(623)$ \\
C - D & $/$ & $0.42(629)$ \\
RecA1 + CTD & $0.46(442)$ & $/$ \\
RecA2 & $0.91(127)$ & $/$ \\
\hline * rm
\end{tabular}

* r.m.s.d. in $\AA$

** $\quad \mathrm{C}_{\alpha}$-atoms used for superposition
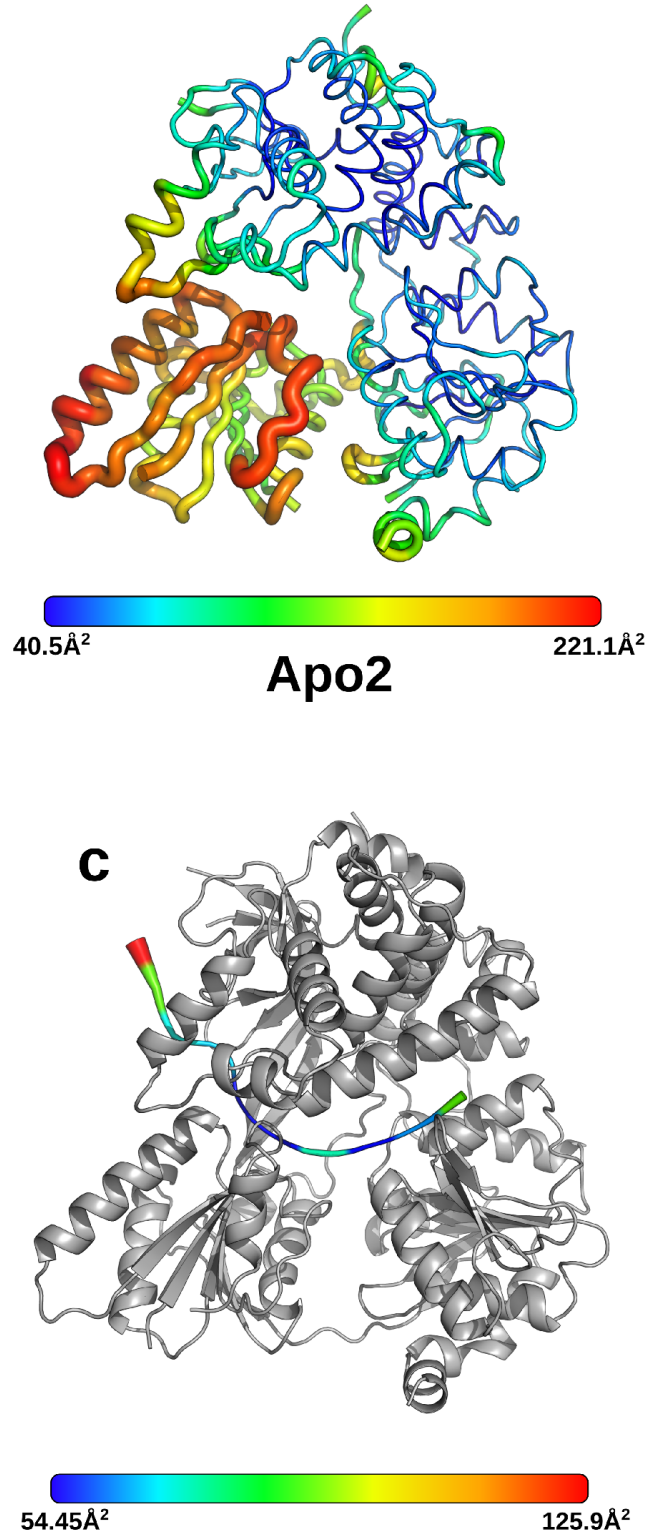

\section{Supplemental figure S3}

$B$-factor distribution of the ctPrp22 structures and r.m.s.d. values of all chains of ctPrp22 Apo and ctPrp22+RNA. (a) The overview representation of the $B$-factors highlights the mobility of the RecA2 domain. (b) All domains of ctPrp22 show an equal distribution of $B$-factors when ssRNA is bound. (c) The ssRNA exhibits only elevated $B$-factor values for the most 5 ' phosphate but consistently low values for the RNA-nucleotides integrated in the binding tunnel. (d) List of r.m.s.d. values of pairwise alignment of all chains in ctPrp22 Apo and ctPrp22+RNA crystal structures. 
a
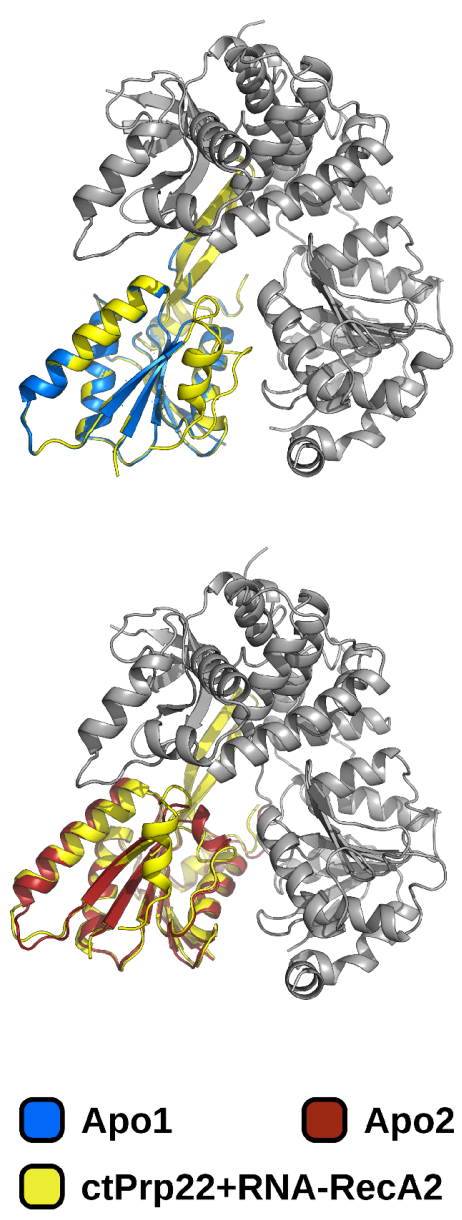
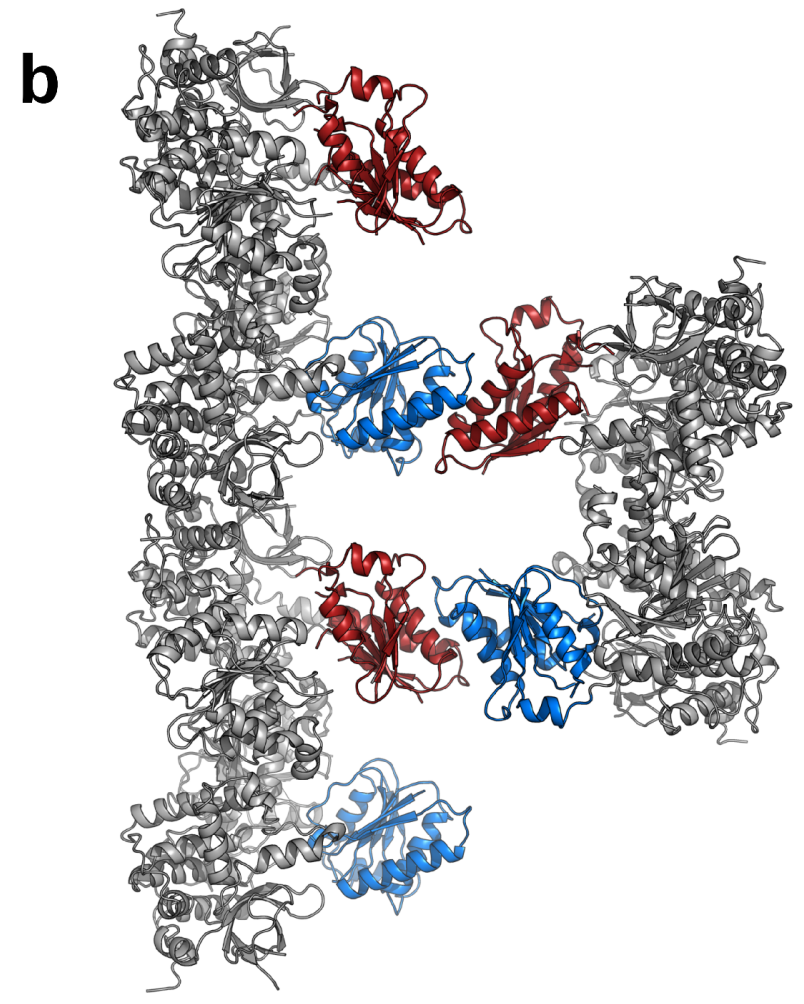

Apo1

Apo2

\section{Supplemental figure S4}

Mobility of RecA2 domain. ctPrp22 models are depicted as in figure 3a. Due to the property of the RecA2 domain to move flexibly as a rigid body, $B$-factors were elevated to a degree that did not allow to build $a$ complete model of this domain. (a) shows a superposition of the complete RecA2 domain of the ctPrp22+RNA complex (yellow) on the RecA2 domains of both Apo molecules. (b) The crystal packing of the ctPrp22-Apo structure shows that the RecA2 domain undergoes the majority of its crystal contacts with the RecA2 domain of the symmetry related molecule. This does not lead to an effective stabilization of the domain further explaining the observed elevated $B$-factor values. 
a
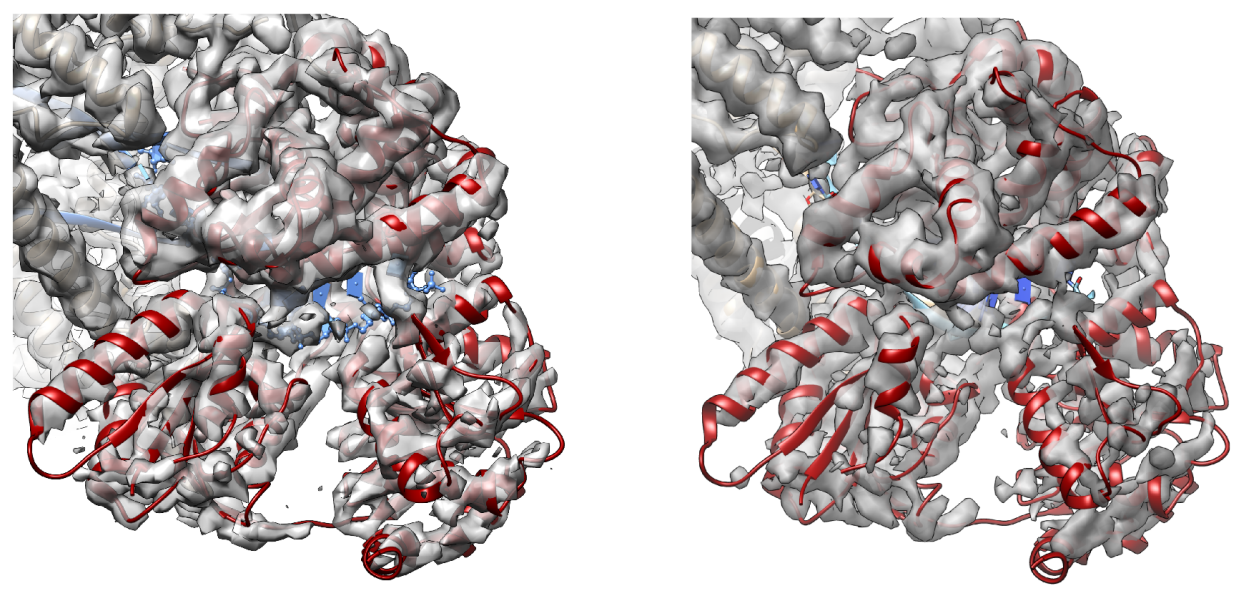

1 ctPrp22+RNA

(EMDBid: 7109)
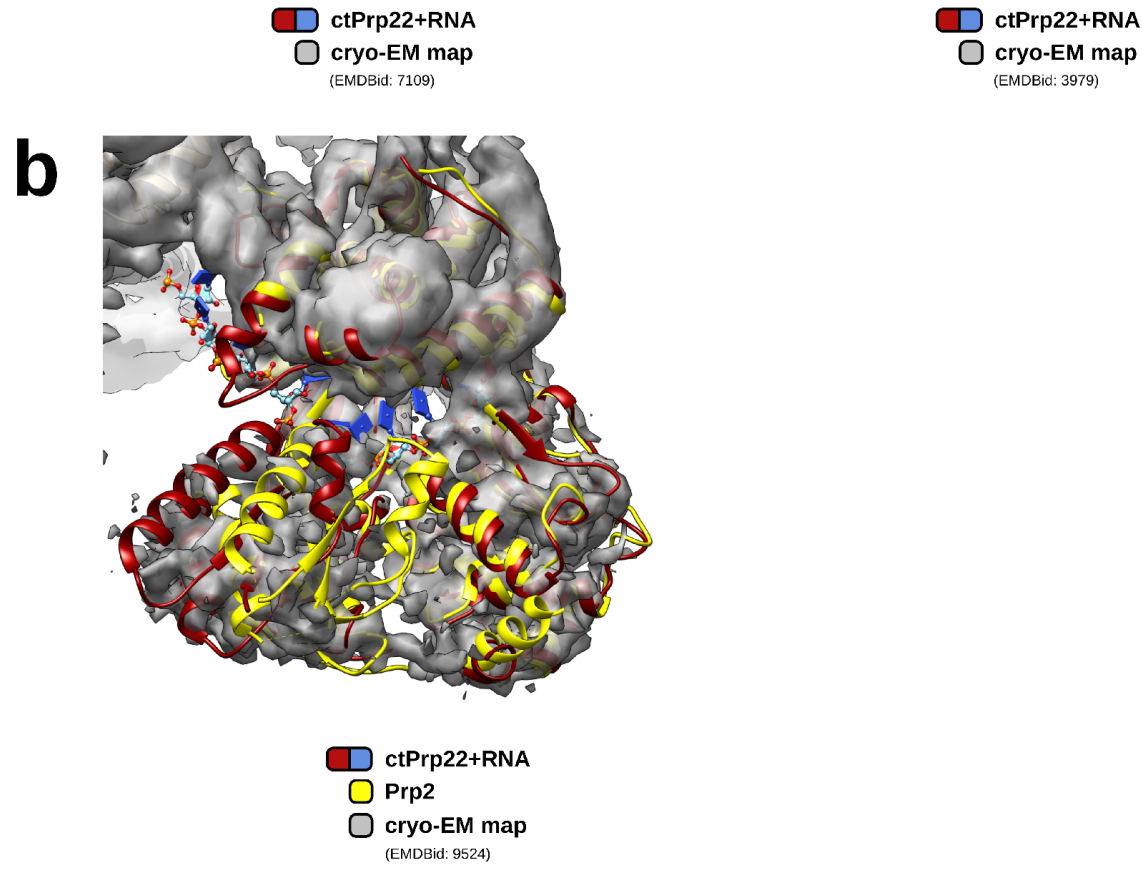

Supplemental figure $\mathrm{S} 5$

Fit of ctPrp22+RNA structure into cryo-EM maps of the yeast spliceosomal postcatalytic $P$ and $B{ }^{\text {act }}$ complexes. The open conformation of the RNA-bound (blue) ctPrp22 (red) complex fits well the data of the yeast spliceosomal postcatalytic $P$ complexes (a) (Liu et al., 2017; Wilkinson et al., 2017) and $B^{\text {act }}$ (b) (Wan et al., 2017) complex helping to assign a specific catalytic state of the ATPase for these cryo-EM structures. 
ADP-bound state

$\mathbf{a}$

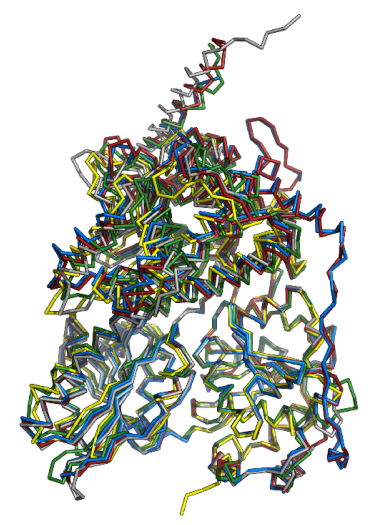

ctPrp43+ADP (PDBid: 5d0u)

scPrp43+ADP (PDBid: 3kx2)

scPrp43+CDP (PDBid: 5jpt)

hsPrp43+ADP (PDBid: 5xdr)

$\bigcirc$ ctPrp2+ADP (PDBid: 6fa5)

\section{RNA-bound state}

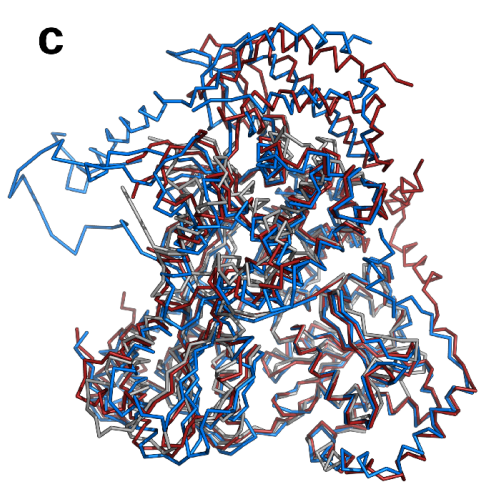

ctPrp22+RNA (PDBid: 6i3p)

dmDHX36+RNA (PDBid: 5n94)

bsDHX36+G-quadruplex (PDBid: 5vhe)

\section{ATP-bound state}
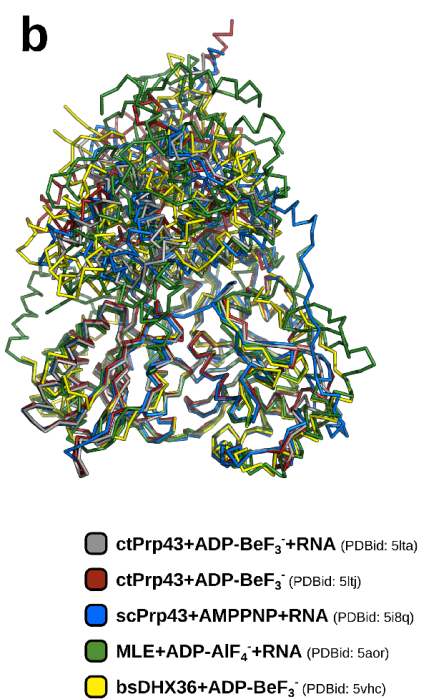

nucleotide-free state

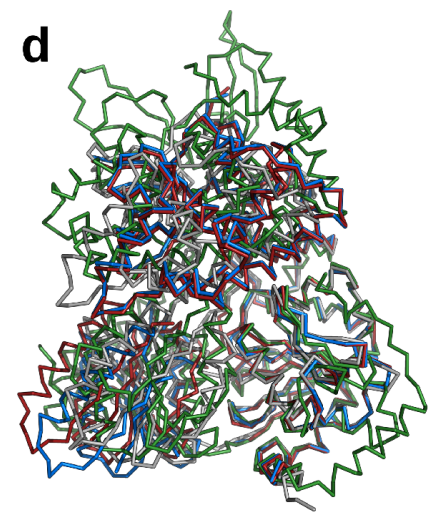

$$
\begin{aligned}
& \text { ctPrp2 (PDBid: 6ta9) } \\
& \text { ctPrp22-Apo2 (PDBid: 6i3p) } \\
& \text { ctPrp22-Apo1 (PDBid: 6i3p) } \\
& \text { bsDHX36 (PDBid: 5vha) }
\end{aligned}
$$

\section{Supplemental figure 56}

Structural comparison of different DExH-box ATPase-specific catalytic states. All structures are superimposed via the RecA1 domain. Structures with bound NDP (a), ATP-analog (b) and only RNA (c) exhibit a highly conserved helicase core conformation that is specific for each catalytic state. In contrast, adenosine nucleotide- and RNA-free structures (d) show divergent positions of the RecA2 domain. 


$$
\text { ctPrp43 + ATP + RNA }
$$

a

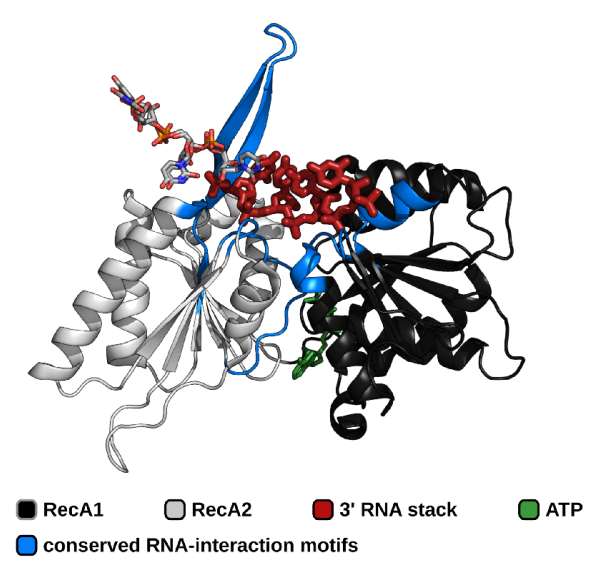

ctPrp22 + RNA

b

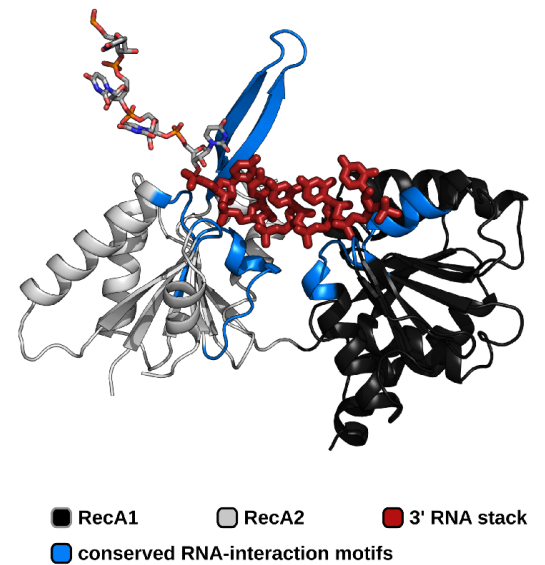

C
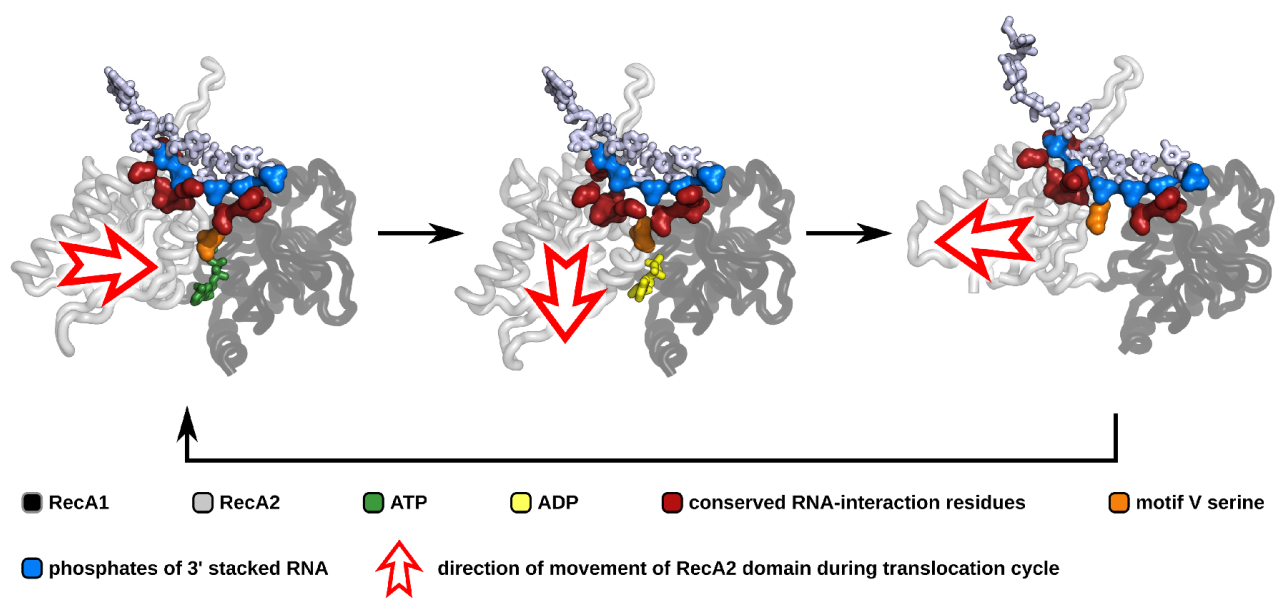

Supplemental figure $\mathbf{5 7}$

d

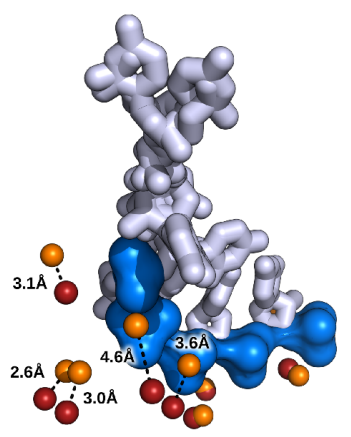

phosphates of $3^{\prime}$ stacked RNA

$\mathrm{C}_{\mathrm{\alpha}}$-atoms conserved RNA-interaction residues ADP-state

$O \mathrm{C}_{\alpha}$-atoms conserved RNA-interaction residues ATP-state
Structural dynamics of the helicase core during a translocation cycle. (a) The bound four-nucleotide stack (red) is maintained by interactions with conserved sequence motifs [motif la, Ib, IV and V] and structural elements [hook-turn, hookloop and $\beta$-hairpin] (blue) in the closed ATP-bound state. The same interactions bind a five-nucleotide stack in the open adenosine nucleotide-free state (b). (c) The translocation dynamics depend on the movement of the RecA2 domain. ATP (green) hydrolysis induces a rotation of the RecA2 domain that interrupts the interactions of the RecA2 domain (red) with the phosphates (blue) of the four-nucleotide stack. Upon ADP (yellow) release the RecA2 domain gains an increased mobility which allows it to bind again to the RNA shifted by one nucleotide towards the 5 ' end. Closure of this open helicase core conformation is induced by the binding of ATP and the RNA is pushed through the tunnel towards 3' direction. (d) The rotation of the RecA2 domain induced by the transition from ATP to ADP, displaces the RNA-interaction residues of this domain by $2.6-4.6 \AA$. This interrupts the interaction of the RecA2 domain with the RNA-backbone when ADP is bound. 


\section{Chapter 5: Structural basis for RNA translocation by DEAH-box ATPases}

a

\section{DExH-box ATPases}

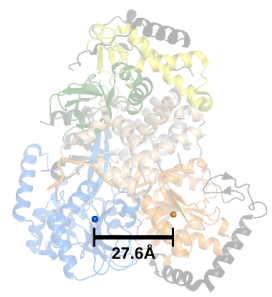

MLE+ADP-AIF-TRA (PDBid: 5aor)

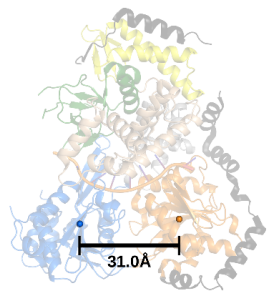

dmDHX36+RNA (PDBid: 5n94)

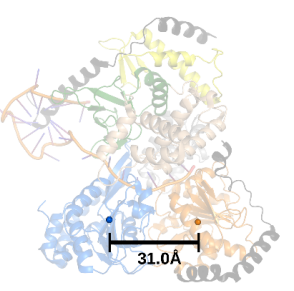

bsDHX36+G-quadruplex (PDBid: 5vhe)

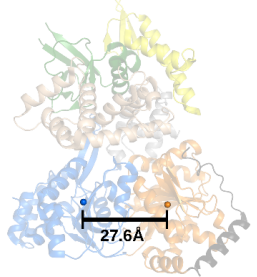

bsDHX36+ADP-BeF (PDBid: 5vhc)

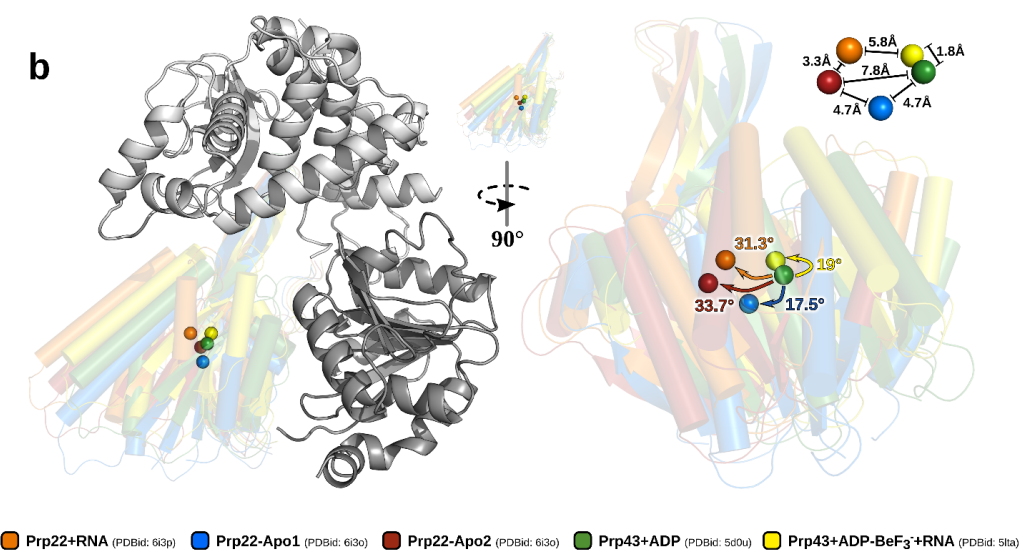

\section{Supplemental figure $\mathbf{S 8}$}

Overview of RecA1-RecA2 domain distances of DExH-box ATPases and RecA2 dynamics. (a) All structures are depicted as semi-transparent cartoon models and colored according to figure 1 . Additional Cterminal domains of DExH-box ATPases are colored in yellow. Center of mass of the RecA-like domains are displayed as spheres and accordingly colored. In order to calculate the centers of mass for the same sets of atoms, the RecA1 and RecA2 domains of the ctPrp22-RNA complex were aligned with the corresponding domains of the individual catalytic states and center of mass for this superposed domains were determined. (b) RecA1 and C-terminal domains of ctPrp22 are depicted as cartoon models in different shades of gray. The RecA2 domains of different DEAH-box specific catalytic states are displayed in different colors (orange: RNA-bound, blue and red: Apo, green: ADP-bound, yellow: ATP- and RNA-bound). The position of the RecA2 domain relative to the RecA1 domain was achieved by superimposing the RecA1 domains of the individual catalytic states. The center of mass of each RecA2 domain is shown as an accordingly colored sphere. In order to calculate the center of mass for the same set of atoms, the RecA2 domain of the ctPrp22RNA complex was aligned with the RecA2 domains of the individual catalytic states and center of mass for this superposed domain was determined. The angles of rotation as well as the translational shifts the RecA2 domain has to undergo are given. 
a

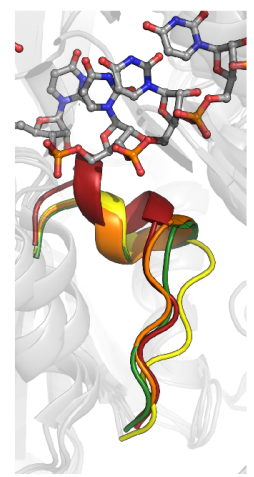

$\bigcirc$ ctPrp22+RNA (PDBid: : 6isp)

ctPrp22+Apo ctPrp43+ADP (PDBid: 5dOU) $\bigcirc$ ctPrp43+ADP-BeF ${ }_{3}+$ RNA b

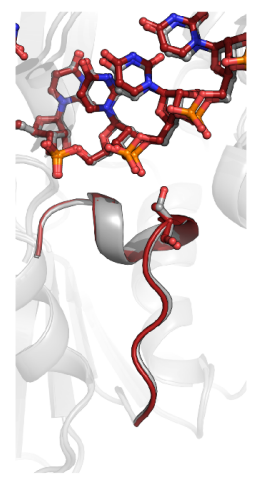

$\bigcirc$ ctPrp22+RNA

ctPrp22-S837A+RN
C

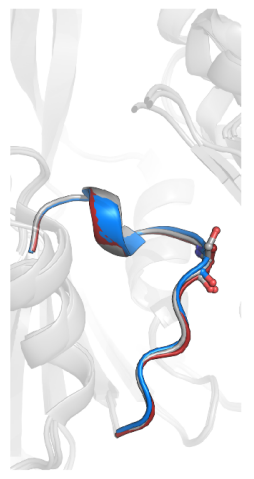

CtPrp43+ADP-BeF

(PDBid: 5tta)

ctPrp43-S387A+ADP-BeF

(PDBid: 6qid) d

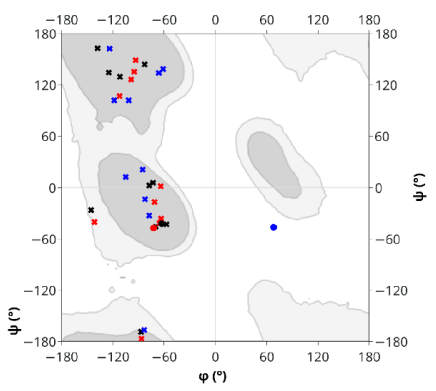

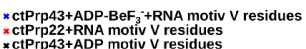

$\times$ ctPrP43+ADP motiv $V$ residues

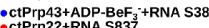

RecA2 overlay
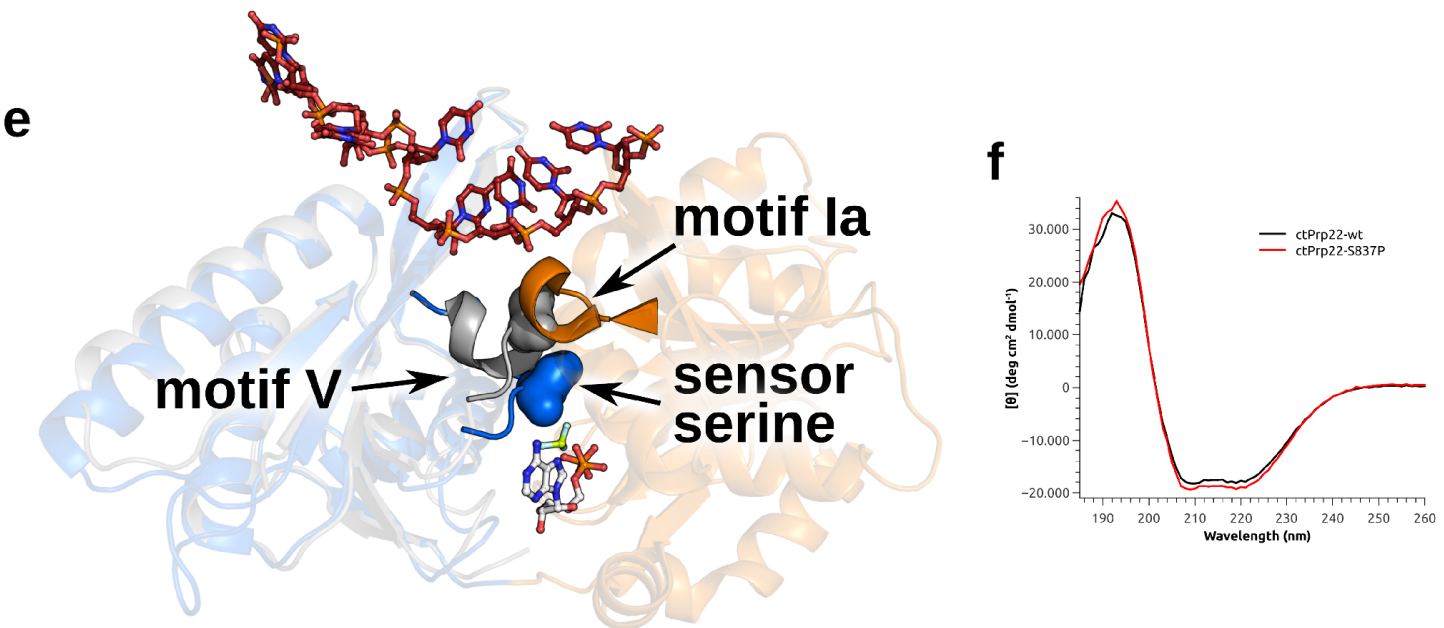

ctPrp43+ADP-BeF ${ }_{3}^{-+R N A}$ (PDBid: 5lta)

ctPrp22+RNA - RecA2 (PDBid: 6i3p)

\section{Supplemental figure $\mathbf{S 9}$}

Motif V conformations. (a) The alignment of different catalytic states of genuine DEAH-box ATPases via the RecA2 domain highlights that motif $V$ always adopts a mostly helical conformation with the exception of the ATP-bound state. There the $\alpha$-helix is heavily distorted mainly due to an extensive interaction network of a serine with numerous components of the active site. Motifs $V$ of ctPrp22-S837A (b), ctPrp43-S387A (c) and ctPrp43-S387G (c) adopt the same adenosine nucleotide-dependent conformations as the corresponding wildtype. (d) Ramachandran plot of motif $\mathrm{V}$ residues (crosses) of the different catalytic states. The motif $\mathrm{V}$ serine (dots) exhibits the most prominent difference as its interactions in the ATP-bound state forces its mainchain into an unfavored conformation. (e) An overlay of the ctPrp22 RecA2 domain of the RNA complex structure (grey) with the RecA2 domain of ctPrp43 in the ATP-bound state (blue) shows that the helical motif $V$ would clash with motif la of the RecA1 domain (orange) in the ATP-bound state. The sensor serine is highlighted as a surface representation. (f) Circular dichroism spectra of ctPrp22 and ctPrp22-S837P are almost identical and confirm the structural integrity of the mutated protein. 

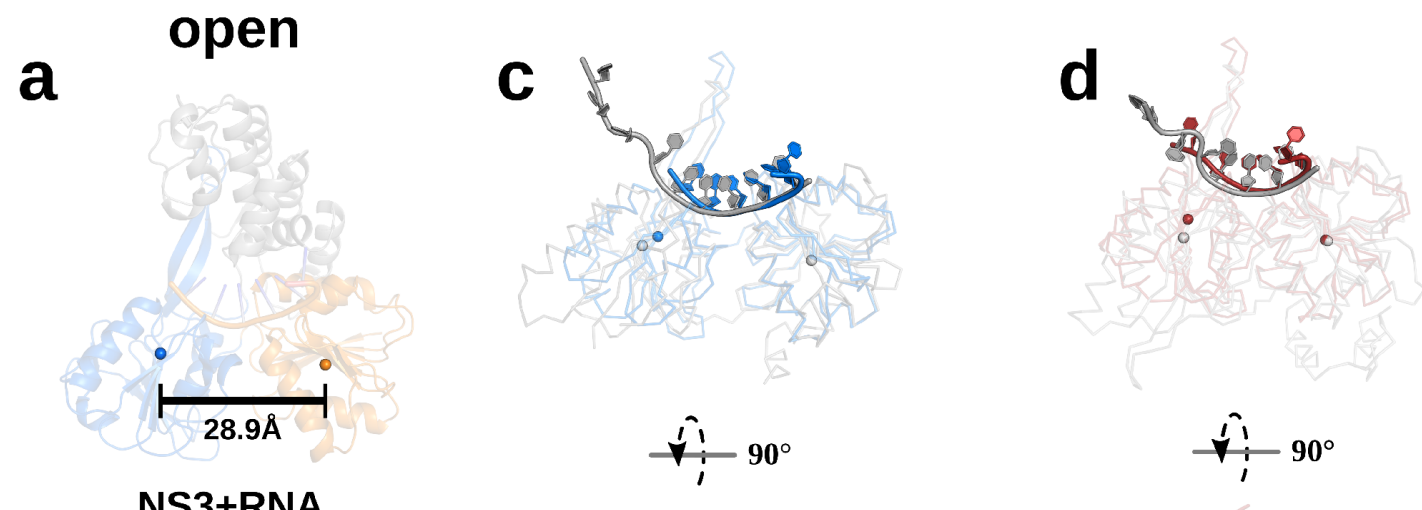

\section{NS3+RNA}

(PDBid: 308c)
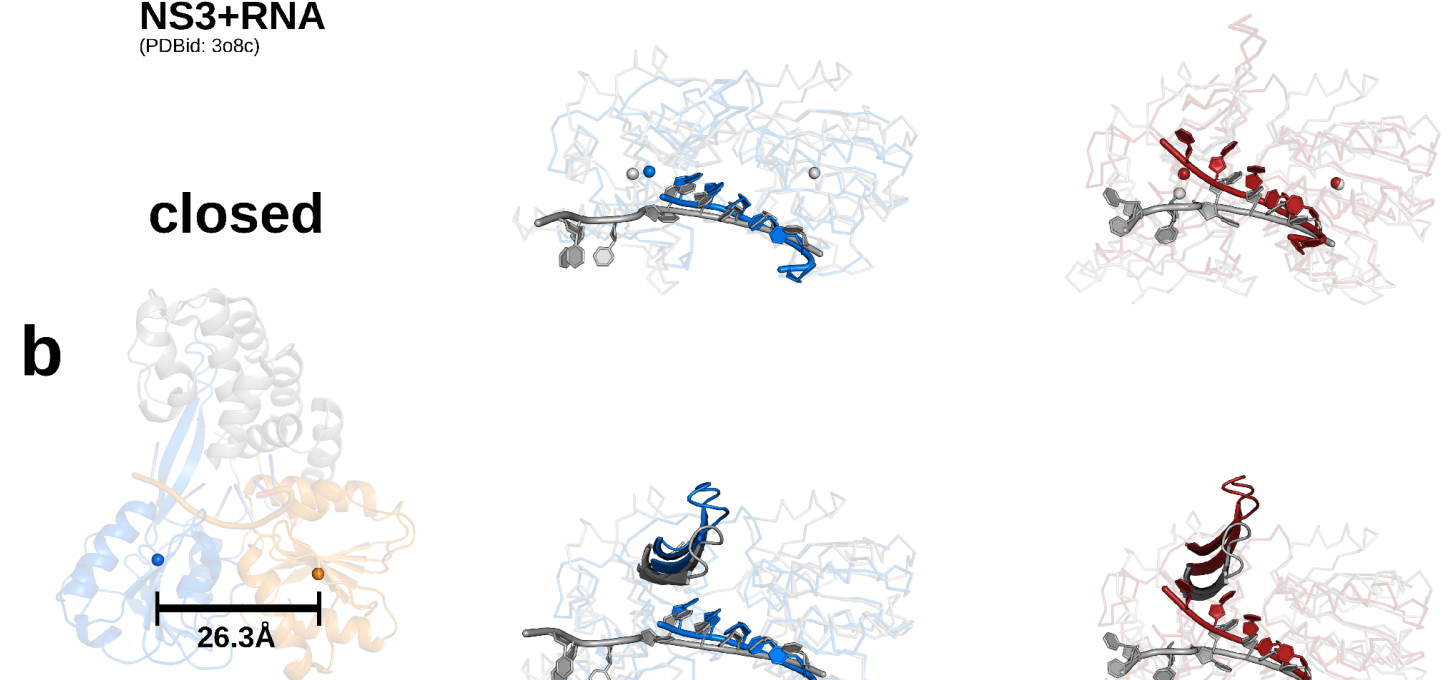

$\underset{\text { (PDBid: 308r) }}{\text { NS3TP+RNA }}$
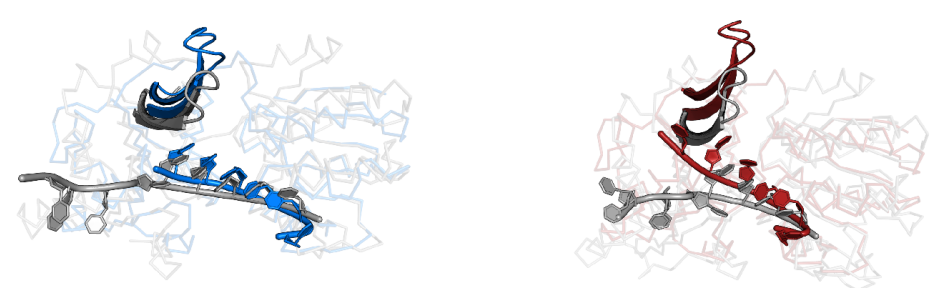

NS3+RNA (PDBid: 308c)

NS3+ATP+RNA (PDBid: 308r)

Prp22+RNA (PDBid: 6i3p)

Prp43+ATP+RNA (PDBid: 5lta)

\section{Supplemental figure $\mathbf{S 1 0}$}

Helicase core conformation in NS3. (a) and (b) show RecA1-RecA2 center of mass distances in the RNAbound and ATP- and RNA-bound states. HCV NS3 depiction and center of mass determination were performed as described in Fig. 3. The helicase core distance is slightly reduced in both states compared to the distances of DEXH-box ATPases. A comparison of the RecA2 domain upon RecA1 domain superposition (c \& d) reveals slightly different RecA2 positioning despite conserved open and close state conformations in DEAH-box ATPases and NS3. This divergent positioning of the RecA2 domain and different interactions with C-terminal domains leads to a different trajectory of the ssRNA through the binding tunnel. 


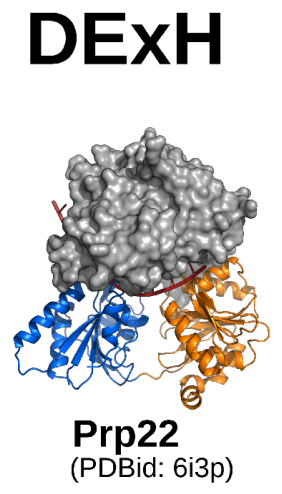

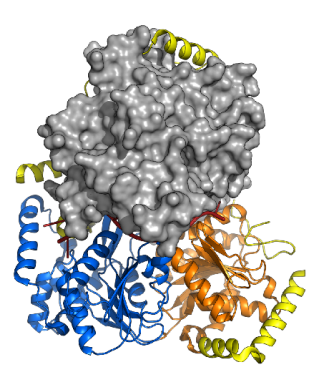

MLE

(PDBid: 5aor)

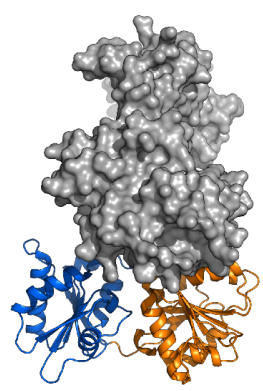

HrpB

(PDBid: 6eud)

\section{NPHII / NS3}

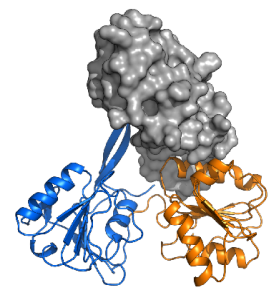

NPHII

(PDBid: 1hei)

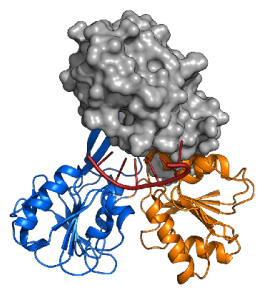

HCV NS3

(PDBid: 308c)

\section{Ski2-like}

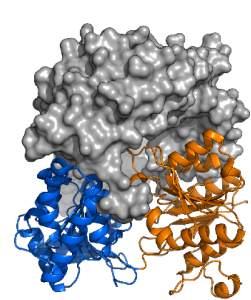

Ski2

(PDBid: 4a4z)

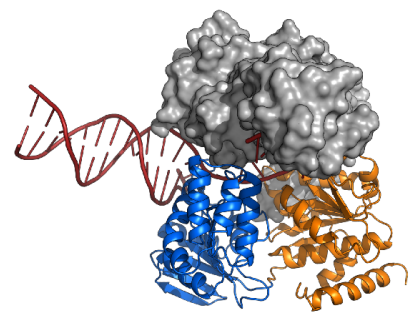

Hel308

(PDBid: 2p6r)

Supplemental figure S11

Overview of C-terminal domains of SF2 helicases. Although the helicase core is highly conserved in SF2 helicases, the C-terminal domains differ significantly in shape, size and composition of subdomains. 


\section{a}
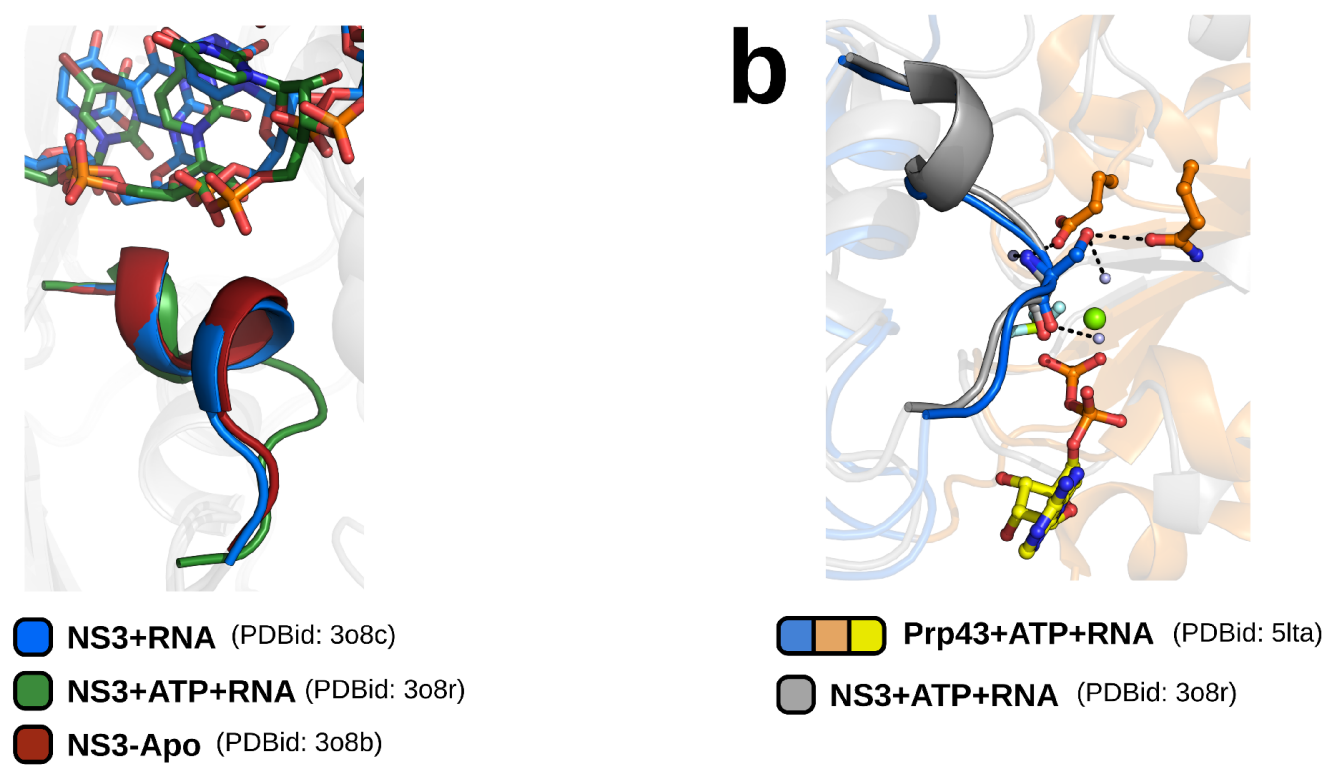

ctPrp22(PDBid:6i3p) ctPrp43(PDBid:5lta) ctPrp2(PDBid:6fa5) bsDHX36(PDBid:5vha) MLE (PDBid:5aor) $\operatorname{HrpB}$ (PDBid: 6eud) NPH - I I (PDBid:1hei) HCV NS3(PDBid:308b) Dengue NS3(PDBid:5xc6) Zika NS3(PDBid:5mfx) Ski2 (PDBid:4a4z) Mt r 4(PDBid:2xgj) Brr2(PDBid:4f91) Hel308 (PDBid:2p6r) Upf1 (PDBid: 2xzl)

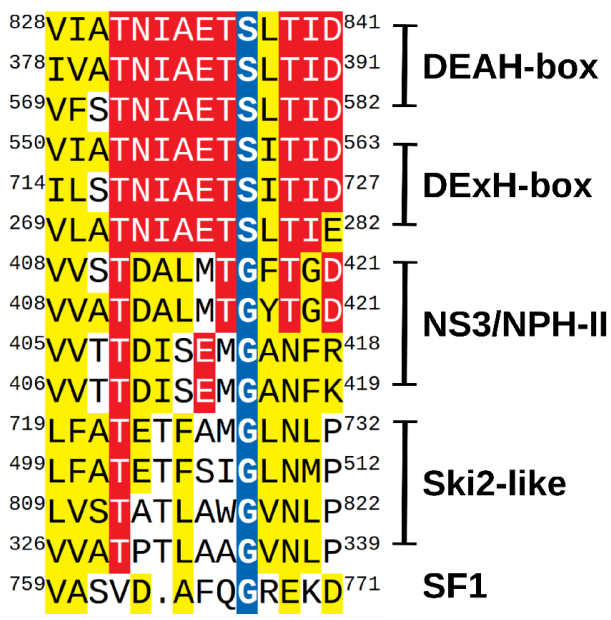

\section{Supplemental figure S12}

Motif V conservation. (a) NS3 motif $V$ also adopts a relaxed helical conformation in adenosine nucleotidefree states and is distorted with bound ATP. (b) Although the motif $V$ serine of DExH-box ATPases is not conserved in NS3 and replaced by a glycine, the overall conformation of the motif in the ATP-bound state is virtually identical. (c) Sequence alignment of motif $V$ in different structurally known SF2 members. The motif $\checkmark$ serine is strictly conserved in DExH-box ATPases but replaced by a conserved glycine in all other SF2 subfamilies. 


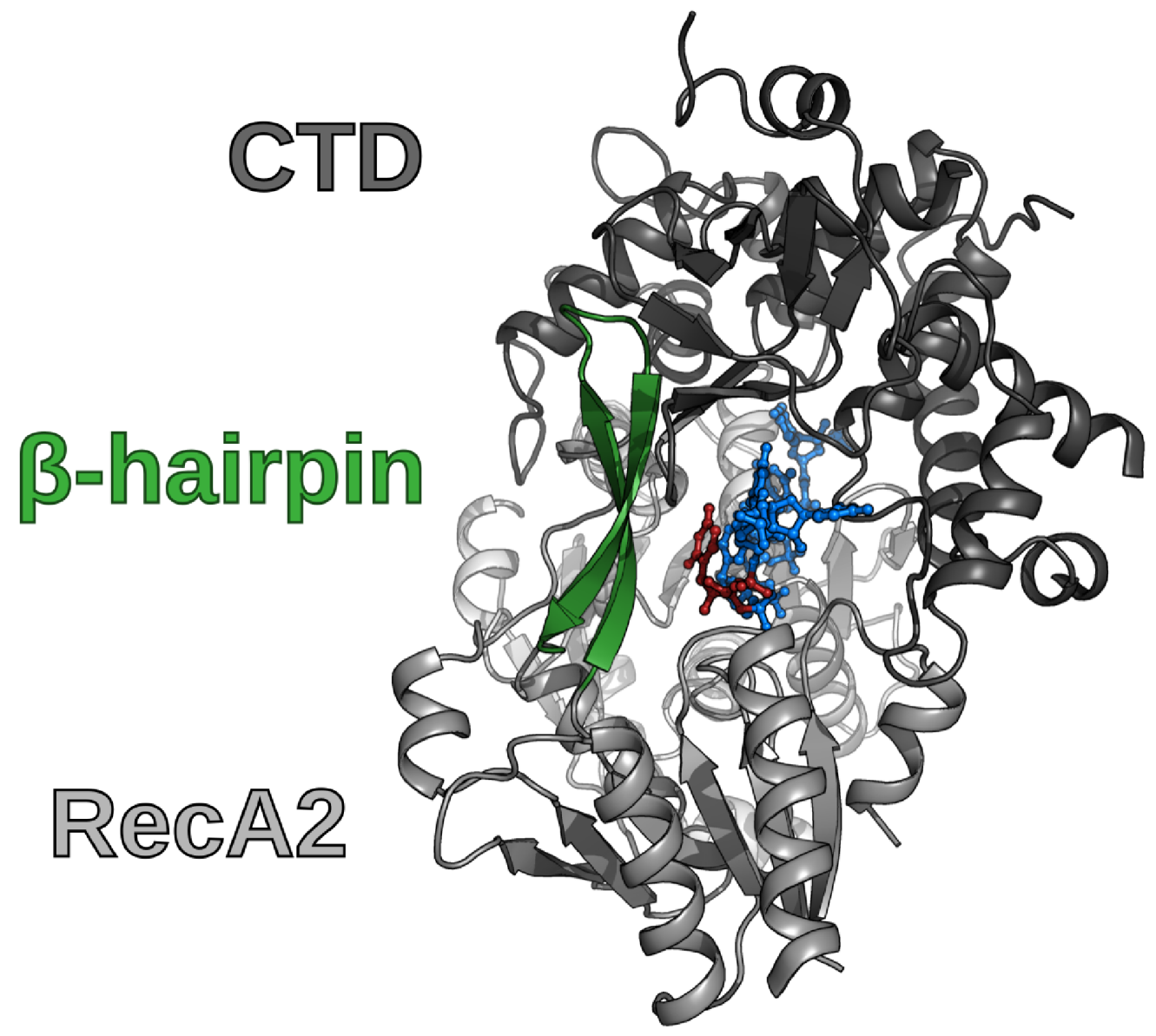

\section{Supplemental movie SM1}

Movement of RecA2 domain incorporates additional RNA-nucleotide in binding tunnel. The morphing of the transition from the ATP-bound ctPrp43 structure towards the adenosine nucleotide-free ctPrp22 structure highlights the movements of the RecA2 domain and its implications on the bound RNA. Due to the opening of the helicase core by the movement of the RecA2 domain, the $\beta$-hairpin (green) is also shifted and creates enough space for the incorporation the next 5' RNA-nucleotide (red) between the hairpin and the four-nucleotide stack of the 3' RNA region. This leads to the stack of five RNA-nucleotides in the adenosine nucleotide-free state. 


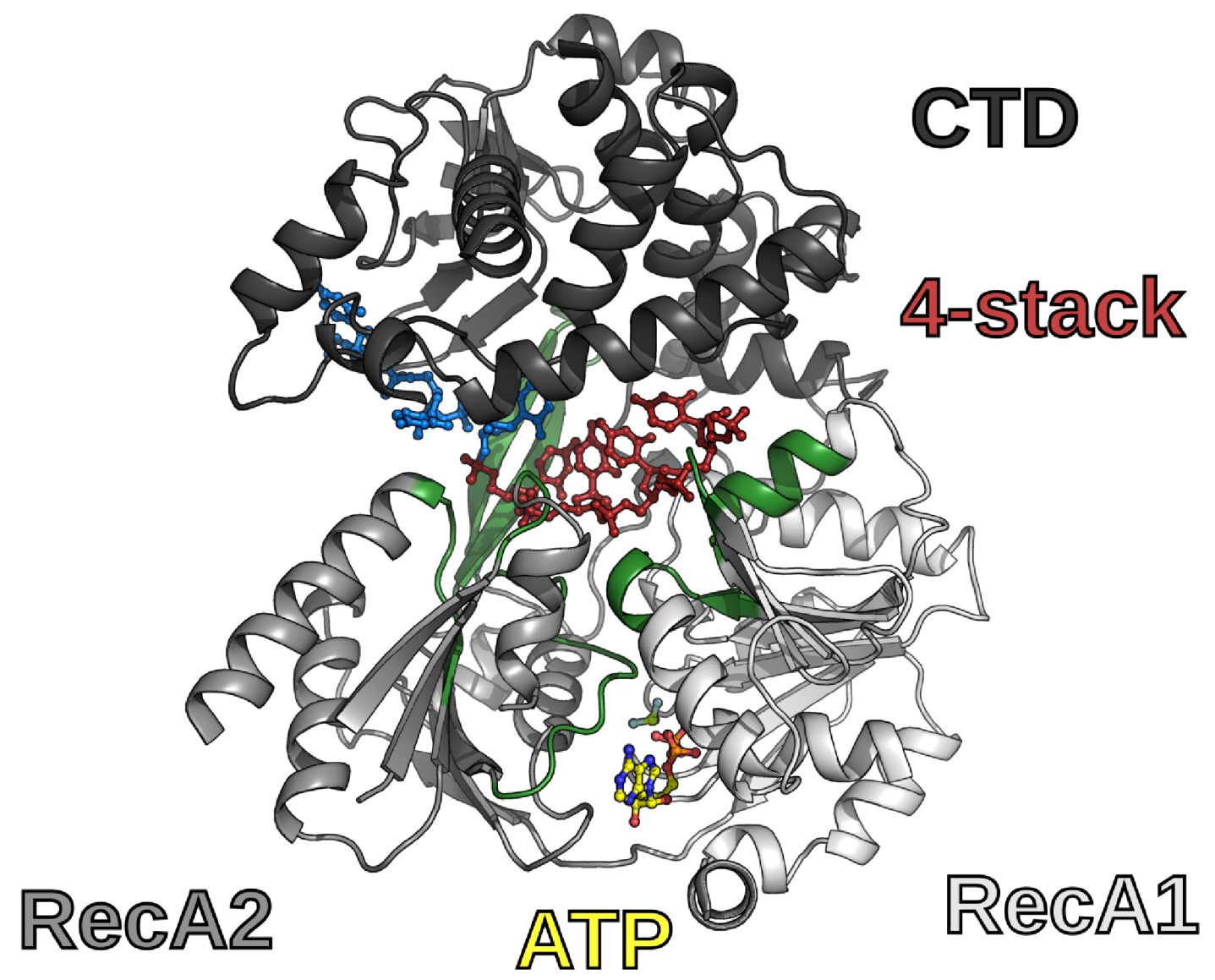

\section{Supplemental movie SM2}

RecA2 dynamics during one step of translocation. A morphing between the ATP-bound (ctPrp43; PDBid: 5lta), ADP-bound (ctPrp43; modeled using PDBid: 5dOu and PDBid: 5 lta) and adenosine nucleotide-free (ctPrp22; PDBid: 6i3p) states illustrates the movements of the RecA2 domain needed for one step of translocation. In the ATP-bound state the conserved RNA-binding sequence and structural motifs (green) bind a stack of four RNA-nucleotides (red) in the binding tunnel. Upon hydrolysis of ATP, the RecA2 domain rotates by $19^{\circ}$ and in the ADP-bound state the RNA-binding motifs of this domain loose the contact to the RNA. After the release of the ADP, the RecA2 domain shifts $6.5 \AA$ towards the 5 ' end of the RNA and binds the RNA shifted by one nucleotide position. This allows the incorporation of one additional RNA-nucleotide in the binding tunnel leading to a bound five nucleotide stack (red). ATP-binding triggers closure of the helicase core and the RecA2 domain is shifted by $5.8 \AA$ towards the RecA1 domain, pushing the RNA through the tunnel. Consecutive cycling of these events allows the DEAH-box ATPases to translocate along an ssRNA in 3'-5' direction. 


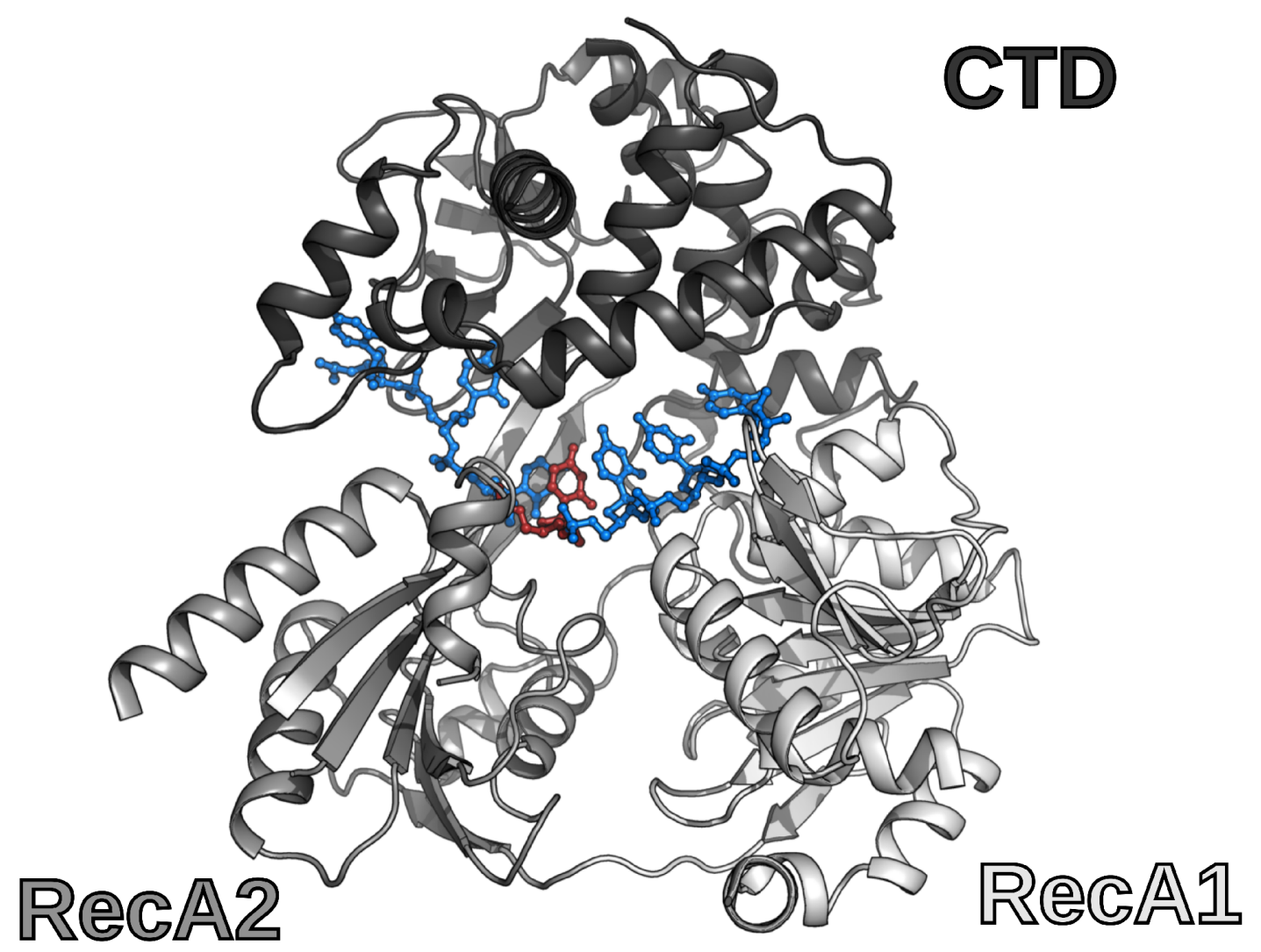

\section{Supplemental movie SM3}

DEAH-box ATPases translocate in 3'-5' direction at a step-size of one RNA-nucleotide per hydrolyzed ATP. Morphing of the transition between the closed ATP-bound conformation of ctPrp43 and the open adenosine nucleotide-free conformation of ctPrp22 in a continuous loop. The highlighted RNA-nucleotide (red) moves through the binding tunnel at a pace of one RNA-nucleotide per closed/open cycle, which represent one ATP hydrolysis cycle. This morphing envisions how DEAH-box ATPases translocate along an ssRNA by moving in 5' direction (3'-5' processivity) at a step-size of one RNA-nucleotide per hydrolyzed ATP. 


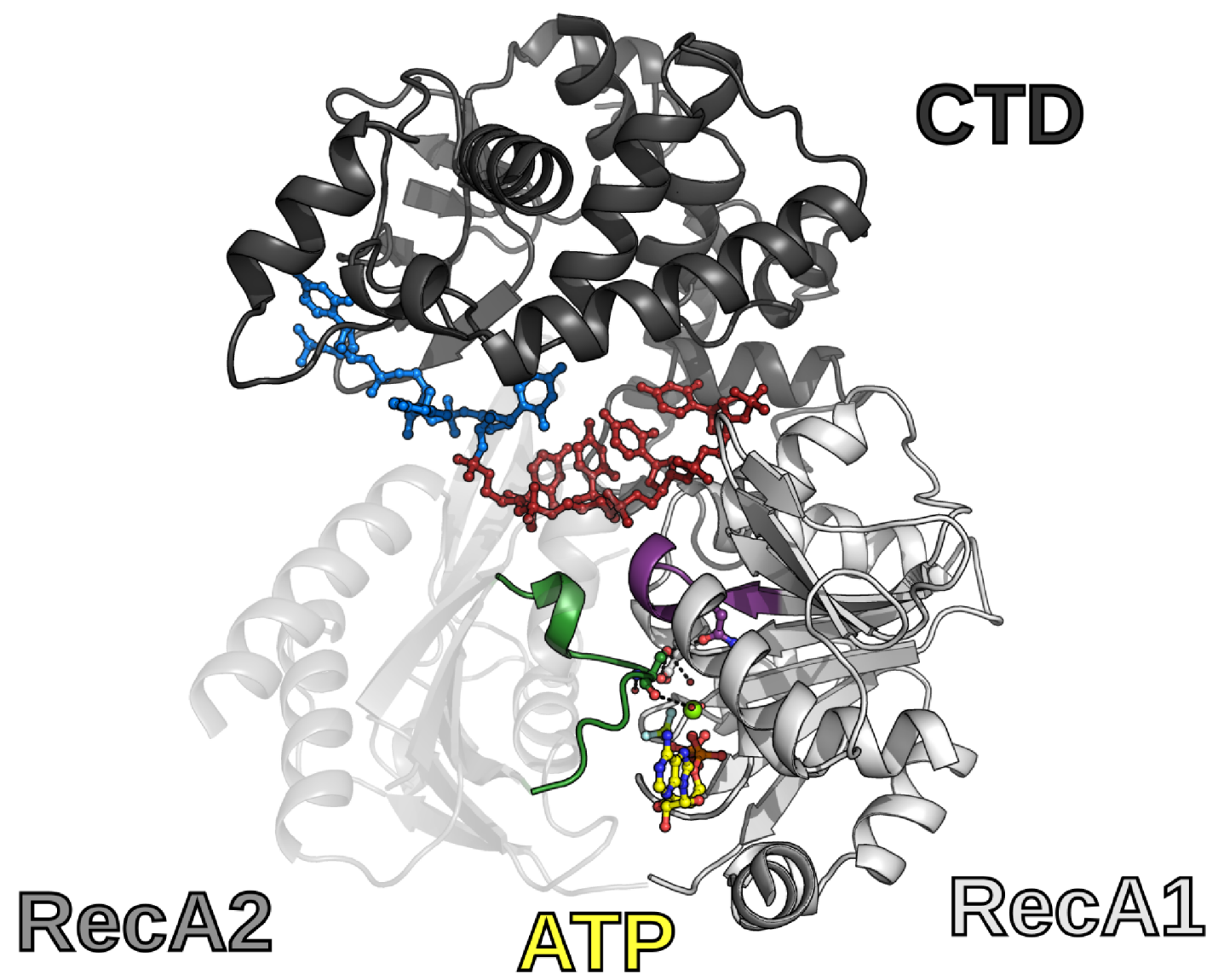

\section{Supplemental movie SM4}

Motif $\mathrm{V}$ senses catalytic state and switches between helical and distorted conformation. Motif $\mathrm{V}$ of DEAH-box ATPases (green) is located in the RecA2 domain and a serine in this motif (ball and stick model) senses the catalytic state by interaction with either the adenosine nucleotides or the ssRNA. Motif $V$ adopts a helical conformation with bound ADP and in the absence of an adenosine nucleotide, which is distorted by the interactions of the serine with conserved active site components in the ATP-bound state. A helical conformation of motif $V$ in the ATP-bound state would clash with motif la (purple) of the RecA1 domain. 


\section{Chapter 6: Discussion and Outlook}

The main goal of the presented work was to unravel mechanistic details about processivity in the DEAH-box ATPase subfamily. Adenosine nucleotide-free structures of Prp22 in absence and in presence of RNA unveiled previously undescribed domain movements that allowed the postulation of a novel model of translocation for this protein family. While the interaction of the ATPases with the 3' stacked RNA region is highly conserved, the 5' RNA region is differently bound in all RNA-containing structures of DEAH-box ATPases. A structure of the ADP- $\mathrm{BeF}_{3}^{-}$- and RNA-bound Prp2 suggests that a protruding loop in the Cterminal domain dictates the conformation of the 5' RNA region and thereby might play a regulatory role that in the case of Prp2 impedes the ATPase from being an unwindase. Catalytic functions of Prp2 are additionally regulated by the interaction with the G-patch protein Spp2. By solving multiple crystal structures of the Prp2-Spp2 G-patch complex the dynamic binding mode could be observed, revealing how the G-patch motif is able to remain constantly bound during the domain movements required for RNA translocation.

While many aspects concerning these results were already discussed in detail in chapters 2-5, the insights gained by the different projects will be put into a broader perspective in this discussion chapter. Here, the conservation of the translocation mechanism as well as the sensing of the catalytic state among SF2 and SF1 members will be in focus. Additionally, more recent structures of DEAH-box ATPases released after the preparation of the publications/manuscripts will be analyzed to provide a revised view of the domain movements as well as of the RNA loading and binding by this protein family. Finally, the importance of translocation by DEAH-box ATPases in the spliceosome will be more throroughly discussed and the newly gained structural insights of the G-patch will be analyzed in the context of the spliceosome.

\subsection{Conservation of translocation in SF1 and SF2 members}

The molecular basis for directional motion by helicases has always been an area of intense interest. During this thesis a set of new structures were determined that shed light onto the RNA translocation mechanism of DEAH-box ATPases (chapter 5). Taking advantage of the variations in conformation as a function of the ligand-bound state, the following translocation model for DEAH-box ATPases could be postulated: In the ATP-bound state the RecA-like domains are in close proximity and accommodate a stack of four RNA nucleotides in the RNA-binding tunnel (He et al., 2017; Prabu et al., 2015; Tauchert et al., 2017). Upon ATP hydrolysis, the RecA2 domain rotates and as a consequence the contacts of this domain with the RNA 
backbone are lost (Schmitt et al., 2018; Tauchert et al., 2016). The release of ADP triggers the opening of the helicase core and the RecA2 domain binds again the RNA backbone shifted by one RNA nucleotide position towards the 5' end leading to a stack of five nucleotide in the RNA-binding tunnel (chapter 5). The binding of ATP ensures the closure of the helicase core and thereby the RNA is pushed through the tunnel in 3' direction by a distance equivalent to one RNA nucleotide. Briefly, the continuous transition between adenosine nucleotide-dependent open and closed states allows DEAH-box ATPases to translocate along an ssRNA at a pace of one RNA nucleotide per hydrolyzed ATP. While it has been discussed in chapter 5 that this model is highly similar to the translocation mechanism proposed for NS3/NPH-II helicases, the closest

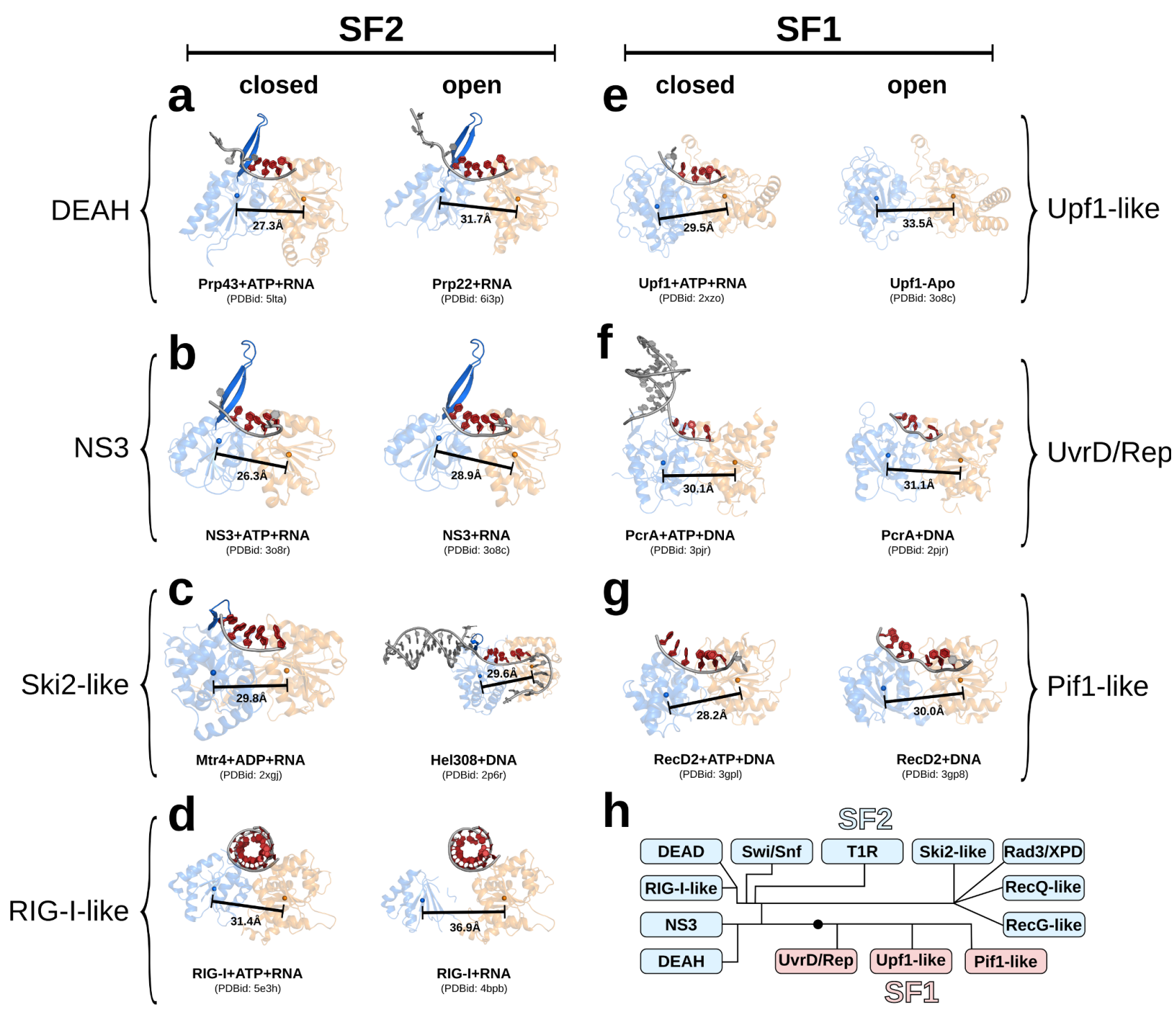

Figure 8: Structural analysis of helicase core dynamics in translocating SF1 and SF2 members. The helicase cores (orange: RecA1; blue: RecA2) of SF1 (a-d) and SF2 (e-g) members known to translocate are shown in ATP-bound and nucleotide-free states, if available. The distance between the domains centers are depicted to highlight the nucleotidedependent movements of the helicase core. Bound nucleic acids are displayed as red and gray cartoon models. Helicase core-induced stacked RNA/DNA regions are shown in red. (h) Cladogram of SF1 and SF2 helicases based on sequence alignments of the helicase core from helicases from E. coli, S. cerevisiae, H. sapiens and several viruses. This figure was adopted from Fairman-Williams et al. (2010). 


\section{Chapter 6: Discussion and Outlook}

of the SF2 relatives, it is of interest if this model can also be applied to more distantly related SF2 or even SF1 members.

Since the key features to ensure the described translocation model are the adenosine nucleotide-dependent opening and closing of the helicase core and the transition between a four and a five RNA nucleotide bound stack, structures of other SF2 and SF1 members proposed to rely on translocation have been analyzed in order to prove similar dynamics (Fig. 8a-g). Since for the DEAH-box ATPase subfamily the most prominent domain movements are observed when comparing ATP-bound and adenosine nucleotide-free states, structures of these two states from a representative of each of the other subfamilies were considered, if available. Among the SF2 members, NS3, Ski2-like and RIG-I-like helicases are known to rely on processivity for their functions (Appleby et al., 2011; Büttner et al., 2007; Gu and Rice, 2010). NS3 helicases are the closest SF2 relatives of the DEAH-box family and the high similarity concerning the translocation mechanism has been thoroughly discussed in chapter 5 (Fig. 8b). Unfortunately, there are no structures of one Ski2-like representative in both catalytic states with bound nucleic acids and therefore two different members of this family in the same state were chosen for a comparison with the DEAH-box and NS3 members (Fig. 8c) (Büttner et al., 2007; Weir et al., 2010). Although no domain movement can be described, these structures show similarities with the nucleotide-free state. Both exhibit a rather open conformation with a comparable RecA1-RecA2 distance to the DEAH/NS3 in the open state along with a bound stack of five nucleotides. Additionally, in the Ski2-like family the 5' end of the stack is delimited by a $\beta$-hairpin, which is also the case for DEAH/NS3. These similarities suggest, that the translocation mechanism proposed for DEAH/NS3 might also be conserved in Ski2-like helicases. In contrast to the DEAH-box, NS3 and Ski2-like members, the RIG-I-like subfamiliy does not translocate along singlestranded RNA, but on double-stranded helical RNA constructs (Devarkar et al., 2018; Jiang et al., 2011; Luo et al., 2011). Despite the completely different target, the fundamental principle of directional motion might be comparable, since a nucleotide-dependent transition between an open and a closed conformation can as well be observed for RIG-I (Fig. 8d).

Most SF1 helicases have as well been proposed to use translocation to fulfill their functions (FairmanWilliams et al., 2010). Due to their similar architecture of the helicase core, which is also composed of two RecA-like domains, a similar translocation mechanism as for DEAH-box helicases seems to be feasible. By comparing the same catalytic states in the three SF1 subfamilies (Upf1-like, UvrD/Rep, Pif1-like), a nucleotide-dependent movement of the RecA-like domains, which is similar to the one observed for SF2 members, can as well be determined (Fig. 8e-g). Interestingly, ATP-bound states of Upf1 and PcrA also exhibit a stack of four nucleotides and the nucleotide-free state of PcrA a stack of five, which suggests a high compatibility with the SF2 mechanism. Although RecD2 appears to be able to accommodate more stacked nucleotides than all other SF1 and SF2 representatives, with a stack of six DNA nucleotides in the ATP- 


\section{Chapter 6: Discussion and Outlook}

bound state and seven in the adenosine nucleotide-free state, it still exhibits the feature of being able to accommodate an additional nucleotide in the nucleotide-free state (Fig. 8g).

Summarizing, a translocation mechanism based on a toggling between open and closed conformational states of the helicase core seems to be a conserved feature of both, SF1 and SF2. On average, the helicase core opens and closes by about $3 \AA$ during each ATP hydrolysis cycle. Additionally, most examples exhibit a binding of a further DNA/RNA nucleotide in the ATP-free state, suggesting that they all translocate with a step-size of one RNA/DNA nucleotide per hydrolyzed ATP. In fact, for many of these helicases an inchworm-typ of translocation mechanism has been described (Appleby et al., 2011; Büttner et al., 2007; Gu and Rice, 2010; Hamann et al., 2019; He et al., 2017; Saikrishnan et al., 2009; Velankar et al., 1999)

These movements of the helicase core, however, need to be tightly regulated for controlled translocation to happen. In chapter 5 a serine in conserved sequence motif $\mathrm{V}$ was proposed to play a central role in sensing the catalytic state and positioning the RecA2 domain. In the following section the conservation of such a sensing mechanism will be in focus.

\subsection{Conservation of catalytic state sensing and RecA2 positioning}

DEAH-box ATPases sense the catalytic state with the help of a serine in the conserved sequence motif V, which interacts via the mainchain with structurally conserved water molecules of the active site. These water molecules are unique to each catalytic state and due to this, DEAH-box ATPases are able to discriminate between a bound ATP or ADP or no adenosine nucleotide at all (chapter 5). This ultimately results in the defined positioning of the RecA2 domain according to the current catalytic state. In this process, the motif $\mathrm{V}$ switches between helical (ADP, no adenosine nucleotide) and distorted (ATP) conformations. This sensing of the active site water molecules shows a striking similarity to the $\gamma$-phosphate sensing mechanism of switch regions in $\mathrm{G}$ domains of guanine nucleotide-binding proteins (Vetter and Wittinghofer, 2001; Wittinghofer and Vetter, 2011). Here, the hydrolysis of GTP triggers a spring-like conformational change of these switch regions, which is transduced to associated domains leading to large molecular movements like observed for motor proteins as kinesin and myosin (Fig. 9i) (Kull and Endow, 2002; Milburn et al., 1990). Since motif V is highly conserved among SF1 and SF2 helicases, a similar sensing mechanism as for DEAH-box ATPases could be expected for all other members. In order to get more insights into the conservation of this sensing mechanism, structures of SF1 and SF2 members in different catalytic state are compared focusing especially on conformational changes of motif V.

To reveal conformational changes of motif $\mathrm{V}$, the RecA2 domains of the individual catalytic states were superimposed and only the motif V is highlighted (Fig. 9a-h). Strikingly, all ATP-bound structures of SF2 


\section{Chapter 6: Discussion and Outlook}

members exhibit a distorted motif V conformation, whereas the other states show this motif in the helical conformation (Fig. 9d-h). Although DEAD-box helicases do not share the ability of translocation with the other analyzed SF2 helicases, they are known to undergo massive conformational changes of the helicase core during the course of the stationary duplex unwinding process (Pyle, 2008). These domain movements seem also to be controlled by the sensing mechanism of motif $\mathrm{V}$, as it is also distorted in the ATP-bound state (Fig. 9f). Interestingly, the DEAH-box subfamily is the only one to exhibit a serine in the prime sensing position of motif $\mathrm{V}$, but since the sensing is performed by the mainchain a glycine at this position is as well

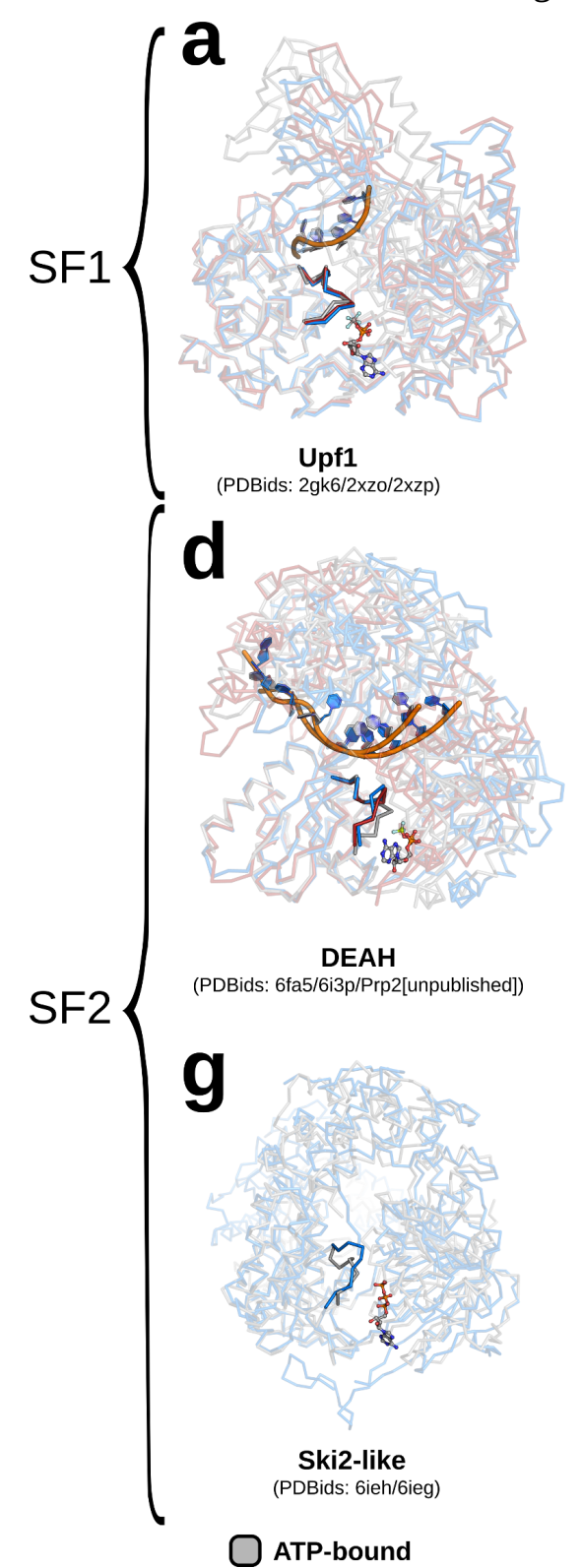

b
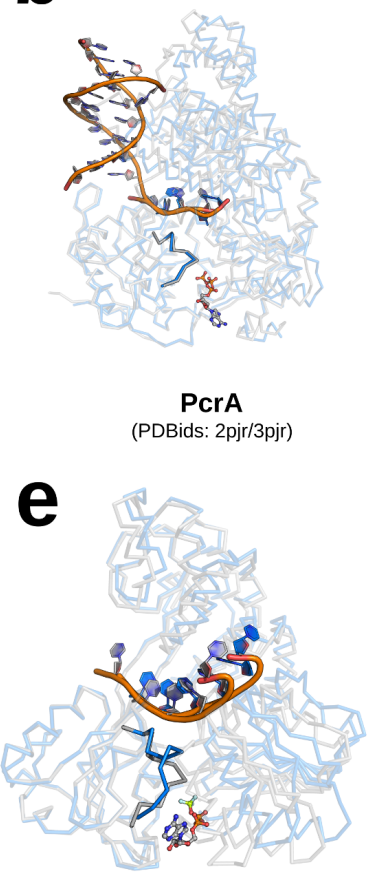

NS3

(PDBids: 308c/308r)

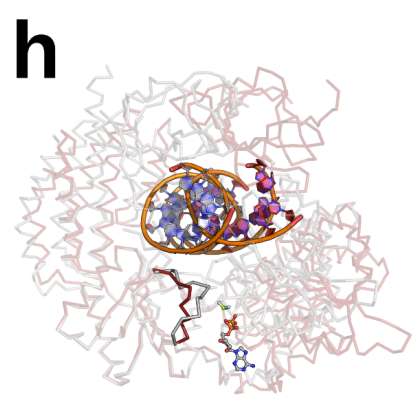

RIG-I

(PDBids: $3 z d 7 / 5 \mathrm{e} 3 \mathrm{~h}$ )

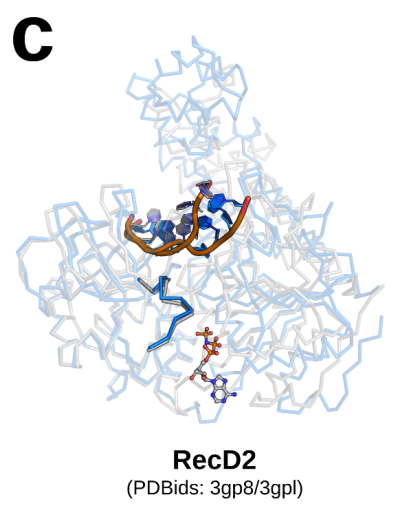

f
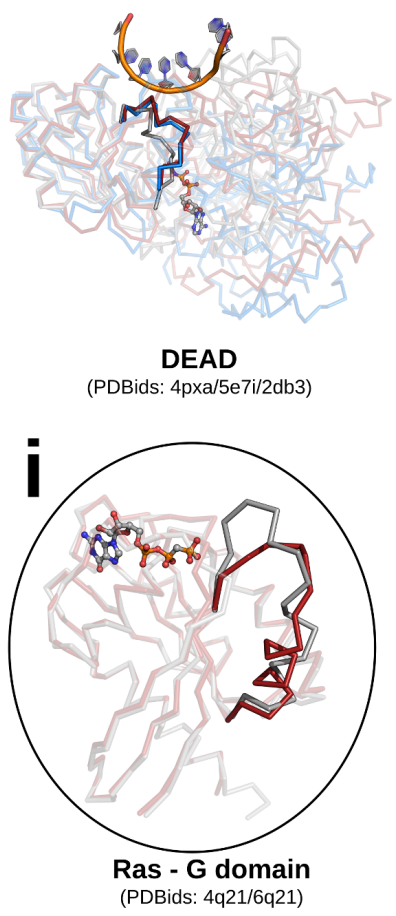

Apo

Figure 9: Structural comparison of the adenosine nucleotide-dependent conformational changes of motif $V$ in $S F 1$ and SF2 families. SF1 (a-c) and SF2 (d-h) helicases are shown as semi-transparent ribbon models in different adenosine nucleotide-bound states. Motif $\mathrm{V}$ is the only region highlighted as non-transparent ribbons. Bound nucleic acids are depicted as cartoon models and adenosine/guanine nucleotides are displayed as ball-and-stick models. (i) The $\mathrm{G}$ domain of Ras is shown as a semi-transparent ribbon model with the switch region highlighted in full opacity. All NTP-bound structures are colored gray, NDP-bound structures red and adenosine/guanine-free structures blue. 


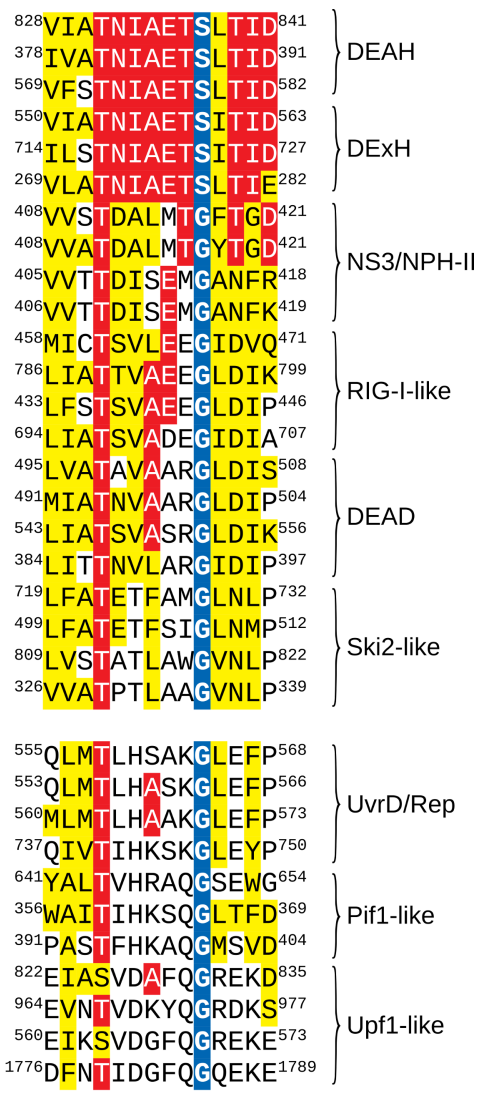

Figure 10: Sequence conservation of motif $V$ in $S F 1$ and SF2 families. The sequence conservation of motif $\mathrm{V}$ is analyzed with respect to the sequence of DEAH/DExH-box ATPases. Residues strictly conserved are highlighted in red and residues sharing similar properties are colored in yellow. The sensor serine/glycine position is depicted in blue.

\footnotetext{
SF1 $\left\{\begin{array}{l}\text { UVrD(PDBid:2is1) } \\ \text { Rep(PDBid:1uaa) } \\ \text { PCrA(PDBid:3pjr) } \\ \text { RecB(PDBid:1w36) } \\ \text { RecD2(PDBid:3gpl) } \\ \text { Pif1(PDBid:5fhf) } \\ \text { Dda(PDBid:3upu) } \\ \text { Upf1(PDBid:2xzo) } \\ \text { Dna2(PDBid:5eax) } \\ \text { Ighmbp2(PDBid:4b3g) } \\ \text { Sen1(PDBid:5mzn) }\end{array}\right.$
}

capable of assuming this function, which is conserved in all other SF1 and SF2 members (Fig. 10). While the motif V seems to be structurally highly conserved in the SF2 family, the sequence conservation is less stringent. Only a threonine at the beginning of the motif and the sensing G/S position are strictly conserved. The variations in sequence of this motif might be necessary to meet the requirements of slightly different domain positions in the other subfamilies or to cope with restrictions from additional auxiliary domains. This might be specially the case for the Ski2-like subfamily, which shows the lowest sequence conservation, as for these helicases the C-terminal ratchet domain has been proposed to play a central role in processivity, while the C-terminal domains in DEAH-box ATPases seem to have a rather regulatory role (chapter 4) (Büttner et al., 2007).

In contrast, SF1 members only show one motif V conformation independently from the catalytic state (Fig. 9a-c). Interestingly, in all structures it is found in the distorted conformation as seen for the ATP-bound state of the SF2 family. The constant identical conformation of the motif indicates that it likely has no sensing function in the SF1 family although the sensor glycine is still conserved (Fig. 10). Here, the primary function of the motif is probably the interaction with the nucleic acids, which agrees with the conservation of a threonine at the beginning of the motif also known to be involved in RNA/DNA backbone interactions in SF2 representatives. In general, the complete motif exhibits a strongly decreased degree of conservation compared with SF2 members. While many hydrophobic residues remain conserved in the SF2 family, these 
are frequently substituted by polar or charged residues in SF1 members. This might give motif V different functional properties in SF1 representatives. However, domain movements for translocation still need to be coordinated and in the SF1 family additional N- or C-terminal domains might adopt the role of sensing and positioning.

In summary, it seems likely that the sensor function of motif $\mathrm{V}$ as proposed for DEAH-box ATPases is conserved across all SF2 members, but not conserved in the SF1 family. Despite the importance of the domain movements of the helicase core for translocation, the dynamics of the auxiliary domains are also of importance. For example movements of the C-terminal domains of DEAH-box ATPases play a role in the loading of the single-stranded RNA. Although many aspects of DEAH-box ATPase domain dynamics have been discussed in chapter 5 , in the next section this topic will be revisited in the light of new structural publications being released after the preparation of the presented publications/manuscripts.

\subsection{DEAH-box ATPase domain dynamics - revisited}

Two recent structural studies about the DEAH-box ATPase Dhr1/DHX37 (S. cerevisiae/M. musculus) have been published since the release of the chapter 5 publication (Boneberg et al., 2019; Roychowdhury et al., 2019). This protein is involved in the eukaryotic ribosome biogenesis, where it catalyzes structural remodeling of RNAs or RNP complexes. Since the Dhr1 structure represents the ligand-free state and the DHX37 structure the RNA-bound but adenosine nucleotide-free state, these structures will be used in this

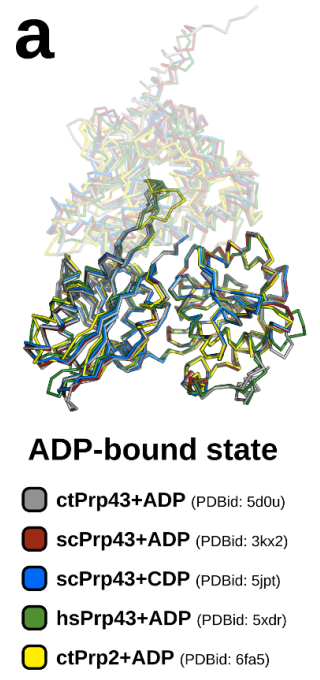

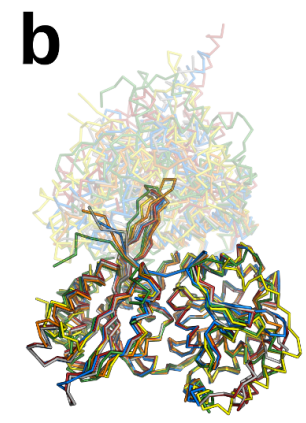

ATP-bound state

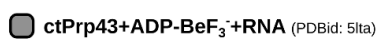

ctPrp43+ADP-BeF ${ }^{-}$(PDBid: 5lit)

scPrp43+AMPPNP+RNA (PDBid: 5i8q)

MLE+ADP-AIF ${ }_{4}^{-}+$RNA (PDBid: 5aor)

bsDHX36+ADP-BeF ${ }_{3}^{-}$(PDBid: 5vhc)

ctPrp2+ADP-BeF ${ }_{3}+$ RNA (unpublished)

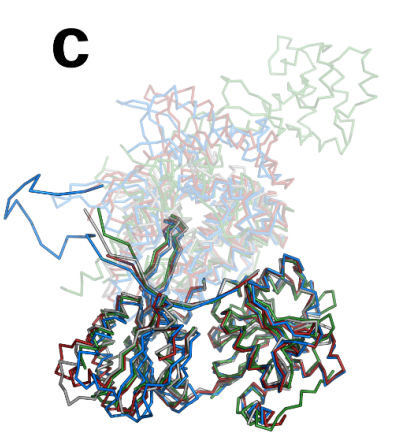

RNA-bound state

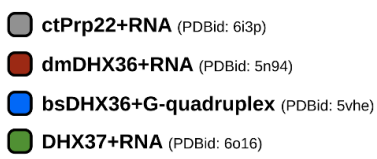

DHX37+RNA (PDBid: 6016)

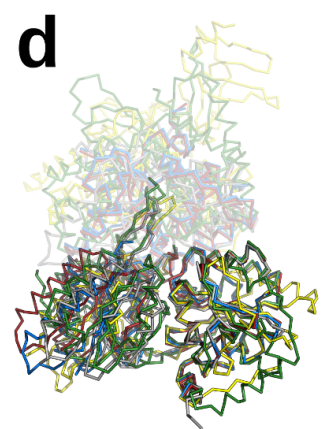

ligand-free state

$\bigcirc$ ctPrp2 (PDBid: 6fa9)

ctPrp22-Apo2 (PDBid: 6i3p)

ctPrp22-Apo1 (PDBid: 6i3p)

bsDHX36 (PDBid: 5vha)

Dhr1 (PDBid: 6h57)

Figure 11: Catalytic state-dependent conformational conservation of the helicase core in DEAH/DExH-box ATPases. DEAH- and DExH-box ATPases are shown as ribbon models with the helicase core highlighted in full opacity. All structure are superimposed via the RecA1 domain. NTP/NDP-bound (a \& b) and RNA-bound (c) structures show highly conserved helicase conformations dependent on the bound ligand. (d) Ligand-free structures exhibit a high variability in the positioning of the RecA2 domain. 
section to reevaluate the helicase core dynamics described in chapter 5 and the C-terminal domain movements needed for RNA loading and binding.

Translocation requires the accurate conformational orchestration of the helicase core, which is induced by the different bound ligands (ATP, ADP and RNA) during this process. Here, the adenosine nucleotide plays the most important role as it is able to dictate the conformation of the helicase core independently of the presence or absence of RNA (Fig. 11b) (Tauchert et al., 2017). The conformation induced by the ATP is highly conserved among genuine DEAH-box ATPases as well as in the closely related DExH-box ATPases, leading to an almost identical superposition of the helicase cores of all available ATP-bound structures. Such a structural conservation can also be observed for the ADP/CDP-bound state (Fig. 11a). While the RNA only plays a subordinate role when an adenosine nucleotide is present, it is responsible for the positioning of the RecA2 domain in absence of any adenosine nucleotide. This leads to a conserved open conformation of the helicase core (Fig. 11c). In chapter 5 this catalytic state of the DEAH-box ATPase Prp22 could only be compared with similar structures of the related DExH-box family, as it was the first structure of a genuine DEAH-box ATPase in this conformation. In the meantime, the structure of the DEAH-box ATPase DHX37

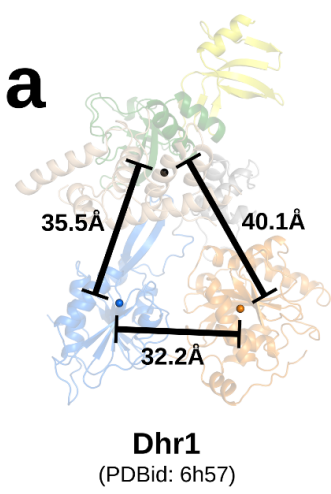

e

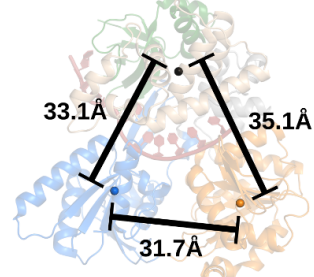

ctPrp22+RNA
(PDBid: 6i3p)

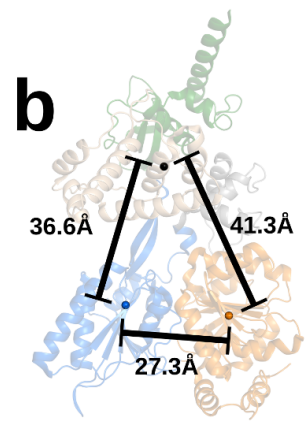

ctPrp43+ADP-BeF ${ }_{3}^{-}$ (PDBid: 5ltj)

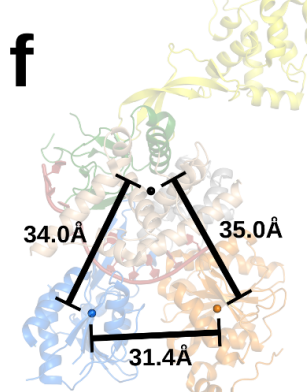

DHX37+RNA

(PDBid: 6016)

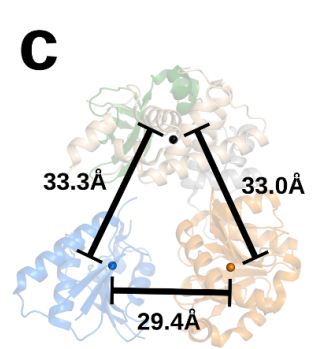

ctPrp22-Apo1 (PDBid: 6i3o)

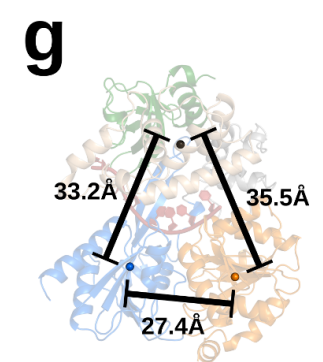

ctPrp2+ADP-BeF ${ }_{3}^{-}+\mathrm{RNA}^{-}$ (unpublished)

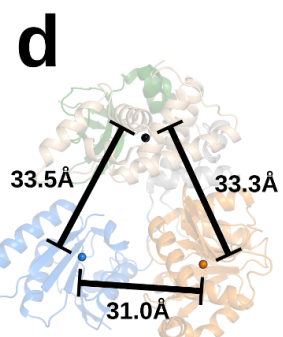

ctPrp22-Apo2 (PDBid: 6i3o)

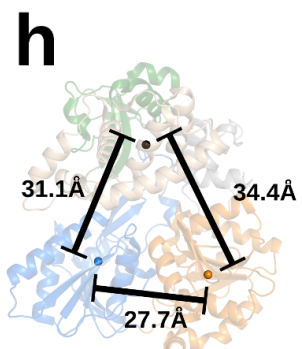

ctPrp2+ADP (PDBid: 6fa5)

Figure 12: Overview of domain distances of different DEAH-box ATPase catalytic states. DEAH-box ATPases are depicted as semi-transparent cartoon models. Center of mass of RecA1 (orange), RecA2 (blue) and the C-terminal domains (black) are shown as spheres. The distances between the centers of mass of these domains are given. The different ligand-bound catalytic states dictate the position of the individual domains, which is represented by varying interdomain distances. 


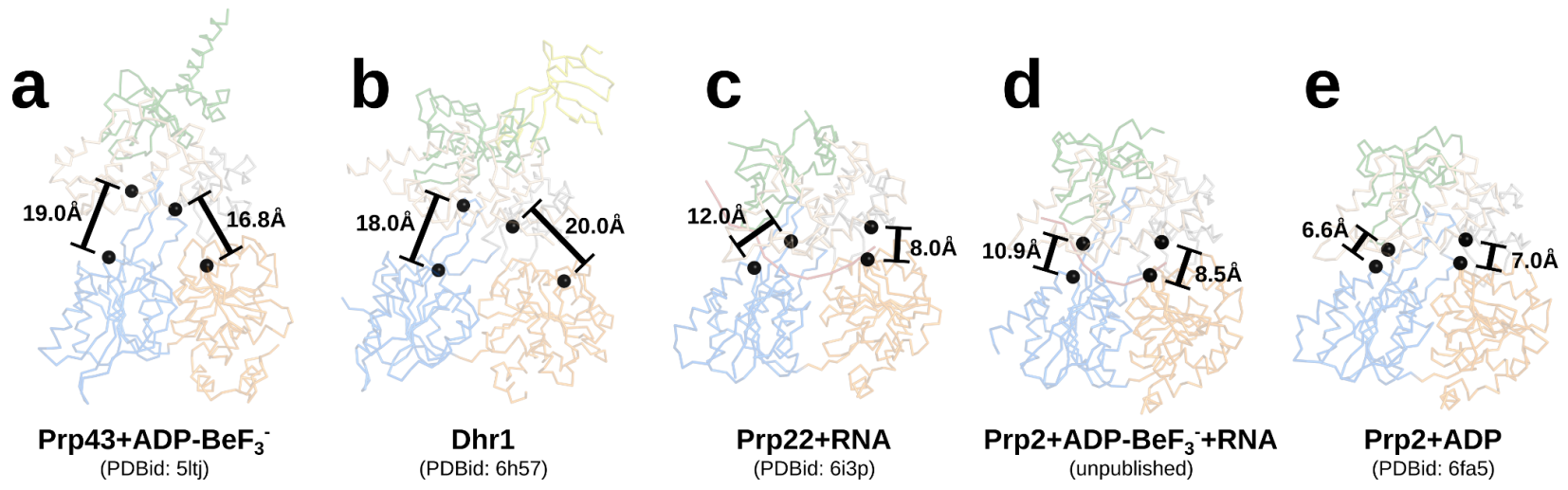

Figure 13: Distance of C-terminal domains to the helicase core in different catalytic states. DEAH-box ATPases in different catalytic states are shown as semi-transparent ribbon models. The distance of the C-terminal domains to the RecA1 and RecA2 domain is given. The black spheres represent the closest residues between these domains, which were found in the ADP-bound Prp2 structure (e). The same residues were used for the analysis of the interdomain distances in all other structures (a-d).

was also solved in the same catalytic state and exhibits a virtually identical open helicase core conformation, further reinforcing a conservation of this RNA-induced conformation (Fig. 12e, f) (Boneberg et al., 2019).

However, if there is no ligand present, the helicase core exhibits an increased conformational freedom. This was postulated in chapter 5 based on a ligand-free structure of Prp22, in which the RecA2 domain adopts two different conformations in the two molecules present in the asymmetric unit. Although other adenosine nucleotide-free structures of Prp2 and DHX36 have been solved at the timepoint of the chapter 5 publication, these structures resemble adenosine nucleotide-bound structures and should be considered with caution as they might be biased by crystal packing. In fact, crystallization conditions and crystal cell constants are virtually identical to their adenosine nucleotide-bound counterparts (Chen et al., 2018b; Schmitt et al., 2018). This in turn argues in favor of the intrinsic RecA2 mobility, as in this case it can be assumed that the RecA2 domain can be forced into the adenosine nucleotide-mimicking conformation by the crystal packing. A recent structure of the DEAH-box ATPase Dhr1 was also solved in the absence of an adenosine nucleotide and RNA (Roychowdhury et al., 2019). Here, the helicase core also exhibits a unique and open conformation. This observation can be used to confirm the intrinsic mobility of the RecA2 domain, which in the ligand-free state is able to adopt a variety of open and closed conformations. Dhr1 even shows a slightly more open helicase core conformation (32.2 $\AA$ ) than reported for the ligand-free Prp22 (29.4 $\AA / 31.0 \AA$ ) (Fig. 12a, c, d). In total, due to the mobility of the RecA2 domain RecA1-RecA2 distances between $27.4 \AA$ and $32.2 \AA$ can be achieved (Fig. 12).

Interestingly, the Dhr1 not only shows an increased RecA1-RecA2 distance, but it also exhibits an increased distance of the C-terminal domains to the helicase core (Fig. 12a; Fig. 13b) (Roychowdhury et al., 2019). $\mathrm{ADP}-\mathrm{BeF}_{3}{ }^{-}$-bound crystal structures of Prp43 show an open conformation of the C-terminal domains exposing the RNA-binding tunnel and it has been suggested that the binding of ATP triggers this opening and 


\section{Chapter 6: Discussion and Outlook}

thereby facilitates the loading of the single-stranded RNA (Tauchert et al., 2017). However, while Prp43 and Prp22 show indeed a slightly increased affinity towards ssRNA in the presence of the ATP-analog AMPPNP, both ATPases are as well capable of stably bind ssRNAs in absence of an adenosine nucleotide (chapter 5). This suggests that ATP is not strictly necessary for the opening of the C-terminal domains, which is in line with the similarly open conformation of the ligand-free Dhr1 (Fig. 13b). In contrast of stabilizing an open conformation, the ATP might rather increase the mobility of this domain. This could favor the transient formation of open conformations, which were trapped in this state by the crystal packing in the two Prp43 structures. It is also worth mentioning, that an ATP-bound structure of the DExH-box ATPase DHX36 is trapped in a closed C-terminal conformation (Chen et al., 2018a). This argues against the stabilization of an open conformation and supports the idea of an increase in mobility when ATP is present. ATP- and RNAbound structures as well as solely RNA-bound structures of DEAH-box ATPases show unanimously a closed C-terminal conformation with the ssRNA enclosed in the RNA-binding tunnel (Fig. 13c, d) (chapter 4 and 5) (Boneberg et al., 2019; Chen et al., 2018a, 2018b; He et al., 2017; Prabu et al., 2015; Tauchert et al., 2017). Once the RNA is bound, its interactions with the C-terminal domains might ensure the maintenance of the closed conformation.

In summary, the new Dhr1 and DHX37 structures confirm on the one hand that the open RNA-bound catalytic state observed for Prp22 is conformationally conserved and on the other hand they provide more proofs for the intrinsic mobility of the RecA2 domain. Additionally, Dhr1 provides the structural evidence that RNA loading is also possible in the absence of an adenosine nucleotide. The C-terminal domains are able to flexibly move as a rigid body independently of the adenosine nucleotide state, but this mobility might be increased in the presence of ATP. In the spliceosome, DEAH-box ATPase are loaded on single-stranded RNA stretches at the peripheries of the complex and in the next section the mechanisms of RNA loading and translocation will be discussed in the context of the spliceosome.

\subsection{Translocation of DEAH-box ATPases in the spliceosome}

DEAH-box ATPases join their respective spliceosomal complexes very transiently as they are usually displaced as a consequence of their remodeling. Since their involvement during splicing is so short-lived, they can only be recruited to peripheral regions to fulfill their tasks. Although they usually ensure rearrangements by the displacement of nearby splicing factors, their intervention remodels also more buried parts of the spliceosome. Due to the inaccessibility of these regions to the DEAH-box ATPases, their targets need to somehow connect these interior parts with the outer binding sites of the ATPases. Prp2, Prp16 and Prp22 bind the pre-mRNA, 3'exon-intron and mRNA, respectively, on the peripheries, which are all linked 

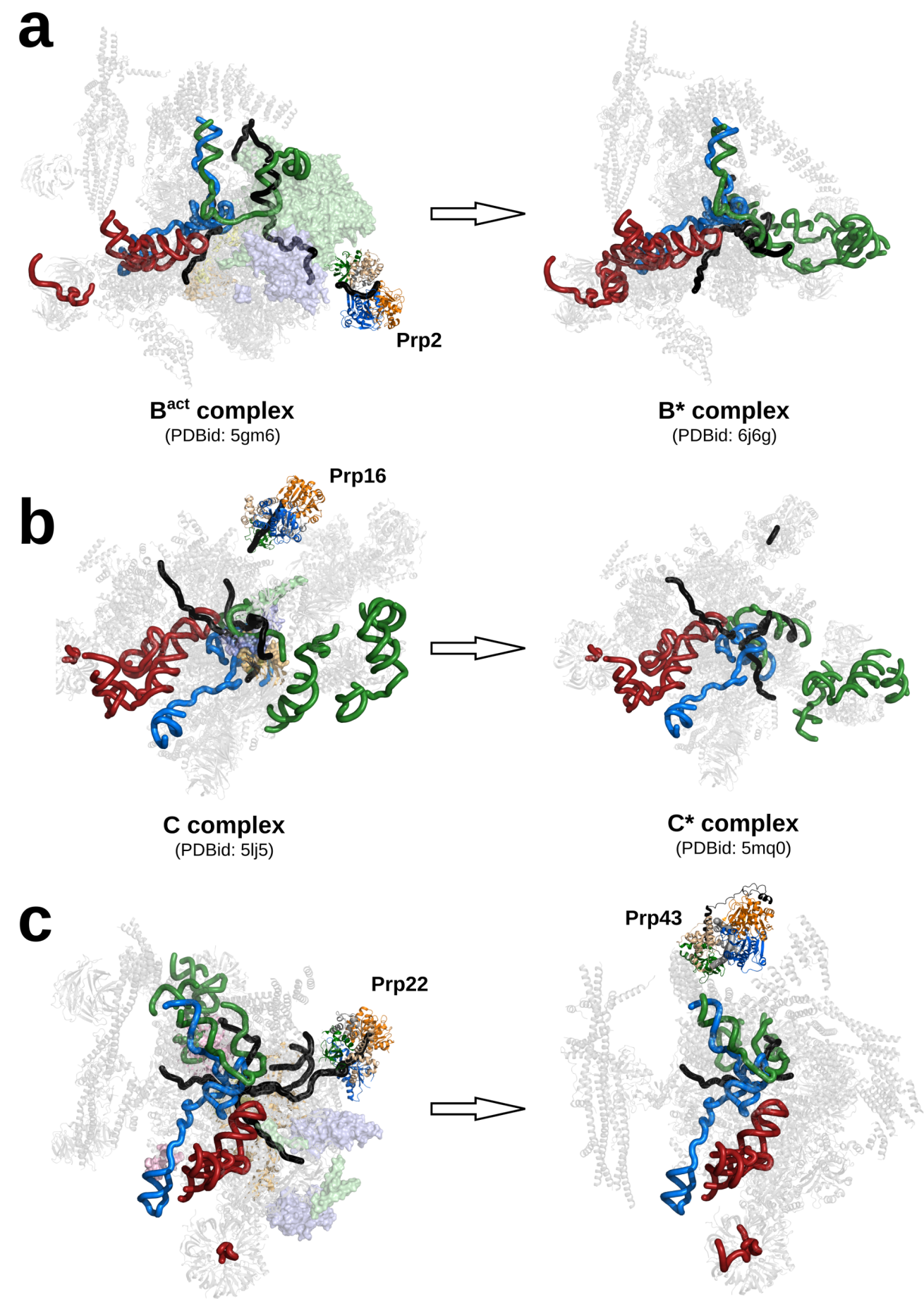

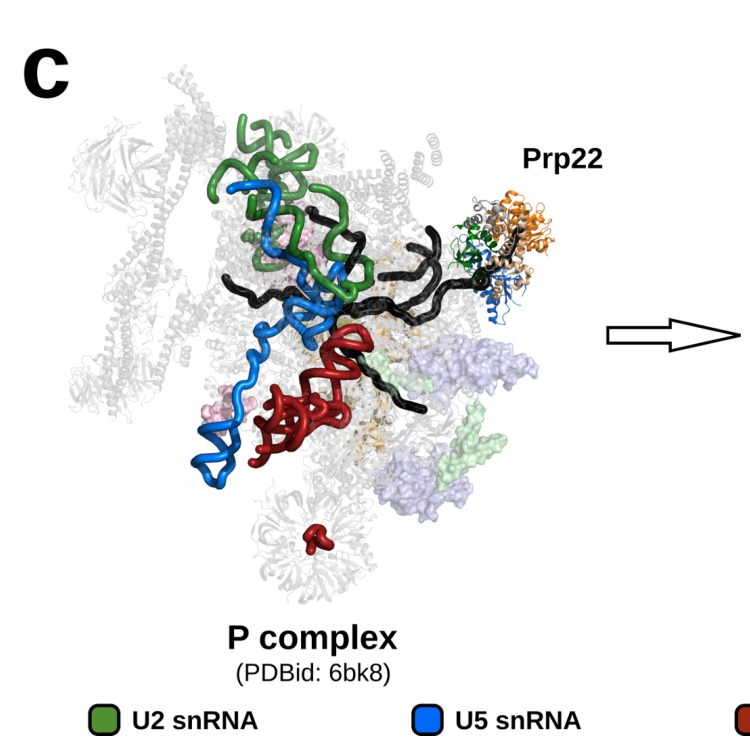

C complex
(PDBid: 5lj5)

U2 snRNA
U5 snRNA
ILS

(PDBid: 5y88)

Figure 14: Effects of DEAH-box ATPase translocation on spliceosomal rearrangements. Protein components of spliceosomal complexes are shown as semi-transparent cartoon models, with the exception of the DEAH-box ATPases, which are shown in full opacity. RNA components are shown as tube models. Displaced splicing factors by the involvement of Prp2, Prp16 and Prp22 are depicted as semi-transparent surface models. DEAH-box ATPases are always located at the periphery of the spliceosome and are facing with the 5' RNA entrance of the RNA-binding tunnel towards the core of the complex. Prp2, Prp16 and Prp22 target single-stranded RNA sequences that are used for translocation. Prp43 is the only ATPase with a duplex nearby. 


\section{Chapter 6: Discussion and Outlook}

to the active site (Fig. 14). By using translocation on these single-stranded RNAs they are able to apply pulling forces on the RNA that have an impact on associated protein factors and on the RNA network.

But before they can fulfill their functions, the proper recruitment of the DEAH-box ATPases is of great importance. Not only do they need to bind the right site at the spliceosome, but need also to be correctly oriented. Since they are 3'-5' translocases, all DEAH-box ATPases are found bound in the spliceosomal cryo-EM structures with the 5' RNA entrance facing towards the core of the complex, from which the target RNA comes from (Fig. 14). In most of these structures the ATPases contact the spliceosome via the Cterminal domains. Such an attachment to the complex provides the spatial requirements for the RecA-like domains to be able to move as needed for translocation. Due to the fact that the DEAH-box ATPases bind to large RNA segments, the loading needs to be performed by the opening of the C-terminal domains, as described in the previous section. Once the RNA is loaded, the translocation of these proteins ensures spliceosomal remodeling.

Prp2 performs translocation on the pre-mRNA and thereby ensures one of the most prominent rearrangements during the splicing cycle. Its involvement displaces the SF3a/b and RES complexes as well as splicing factors Cwc24 and Cwc27 (Wan et al., 2019). In the B ${ }^{\text {act }}$ complex, these factors are arranged close to the pre-mRNA (Fig. 14a) (Rauhut et al., 2016; Yan et al., 2016). The influence of the Prp2 translocation on the pre-mRNA likely influences the interaction of these factors with the pre-mRNA, ultimately leading to their dissociation. But also the RNA network is remodeled. The U2/pre-mRNA duplex is significantly shifted towards the active site in the $\mathrm{B}^{*}$ complex compared to its more outer position in the $\mathrm{B}^{\text {act }}$ complex. Whether this relocation is directly caused by the Prp2 translocation or by the displacement of the splicing factors cannot be assigned with certainty, but it is likely a combined effect. Interestingly, Prp16 has a lower impact on compositional changes in the transition from the C to the C* complex (Fica et al., 2017; Galej et al., 2016). Due to its involvement only Cwc25, Yju2 and Isy1 are displaced (Fig. 14b). These factors are close to the active site and its displacement highlights how DEAH-box ATPases are able to remodel the spliceosome composition from a distance. The pulling on the 3'exon-intron RNA by the Prp16 translocation additionally causes a dramatic $75^{\circ}$ rotation of the active site branch helix, a duplex between $\mathrm{U} 2$ and the intron. In the P complex, Prp22 releases the ligated mRNA from the complex possibly by pulling out the RNA via translocation, which also displaces several other splicing factors nearby (Fig. 14c) (Liu et al., 2017). The active site however remains vastly intact (Wan et al., 2017). Prp43 is the only DEAH-box ATPase in the spliceosome with a duplex in close proximity. It is yet unclear if it targets the U2 or U5 snRNA and it is even feasible, that it is the only spliceosomal DEAH-box ATPase to couple translocation to direct duplex unwinding. The involvement of Prp43 leads to the complete disassembly of the spliceosome. 


\section{Chapter 6: Discussion and Outlook}

Summarizing, the cryo-EM structures reinforce the importance of translocation by DEAH-box ATPases. Due to their transient involvement in the spliceosome they can only be recruited to the peripheries of the complex, but need to ensure the remodeling of distant spliceosomal regions. This is achieved by targeting RNAs at the complex surface that are also part of the active site. The pulling effect of translocation then has an impact on splicing factors associated with this RNA and RNA networks at the active site. While the catalytic functions of Prp22 and Prp16 are not known to be regulated by other spliceosomal factors, Prp2 and Prp43 require the help of G-patch proteins to fulfill their tasks. The interaction of Prp2 with its co-factor Spp2 has been discussed in detail in chapter 3 and in the next section these gained insights will be used to analyze the Prp43 interaction with the G-patch protein Ntr1 in the context of the spliceosome.

\subsection{Insights into the Prp43-Ntr1 interaction in the spliceosome}

The G-patch proteins Spp2 and Ntr1 play a vital role for the DEAH-box ATPases Prp2 and Prp43, respectively (Fourmann et al., 2016; Roy et al., 1995; Silverman et al., 2004; Tsai et al., 2007; Warkocki et al., 2015). By the stimulation of the ATPase activity of both and the helicase activity of Prp43 their interaction with the respective ATPase is essential for the spliceosomal function (Christian et al., 2014; Tauchert et al., 2017; Warkocki et al., 2015). Chapter 3 reveals the first structural insights into the interaction of a G-patch motif with a spliceosomal DEAH-box ATPase based on the complex crystal structure of Prp2 and the Spp2 G-patch. Due to the limited resolution at the peripheries of spliceosomal cryo-EM structures, the structural elucidation of the at least partially disordered G-patch proteins has remained an unsolved challenge. In chapter 3 the crystal structure of the Prp2-Spp2 G-patch complex together with available crosslinking data of the $\mathrm{B}^{\text {act }}$ complex was used to locate the G-patch within the spliceosome and predict the attachment to the spliceosome of flanking Spp2 regions. Using the information gained from chapter 3, the cryo-EM-based information of the interaction of Prp43 with the G-patch protein Ntr1 in the ILS will be revisited in this section (Wan et al., 2017).

Although there is no structural information about the Prp43-Ntr1 G-patch interaction, it has been suggested in chapter 3 that the interaction might be highly similar to the one of Prp2 and Spp2. This was based on the facts, that the G-patch motif and the interaction sites on the DEAH-box ATPases are highly conserved and that available cross-link data support a comparable binding (Christian et al., 2014). Based on this, the Gpatch of Ntr1 can be modeled onto the Prp43 structure in the spliceosome (Fig. 15a). In contrast to Spp2, which is intrinsically disordered in its entirety, Ntr1 exhibits only disorder in its G-patch-containing Nterminal region. The C-terminal part adopts a large superhelical conformation, which is attached to Snu114 at its most C-terminal end (Wan et al., 2017). The model of the Ntr1 G-patch is oriented in a way that it faces 


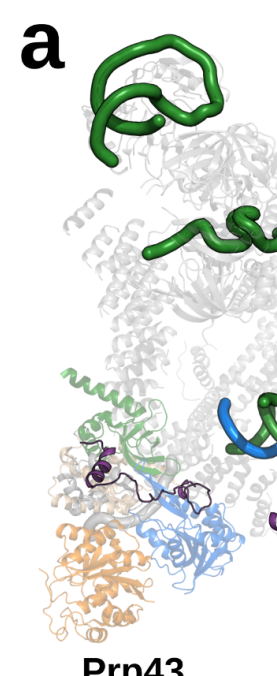

Prp43

$\sin \frac{1}{\infty}$

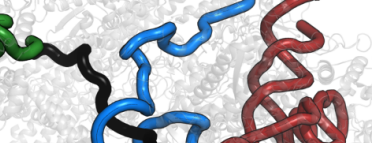
(1)

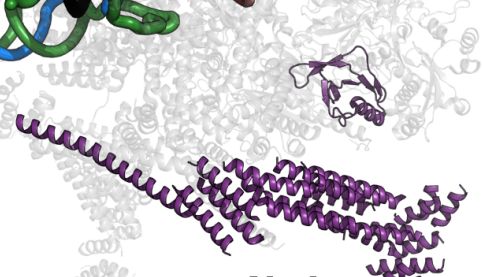

Ntril b

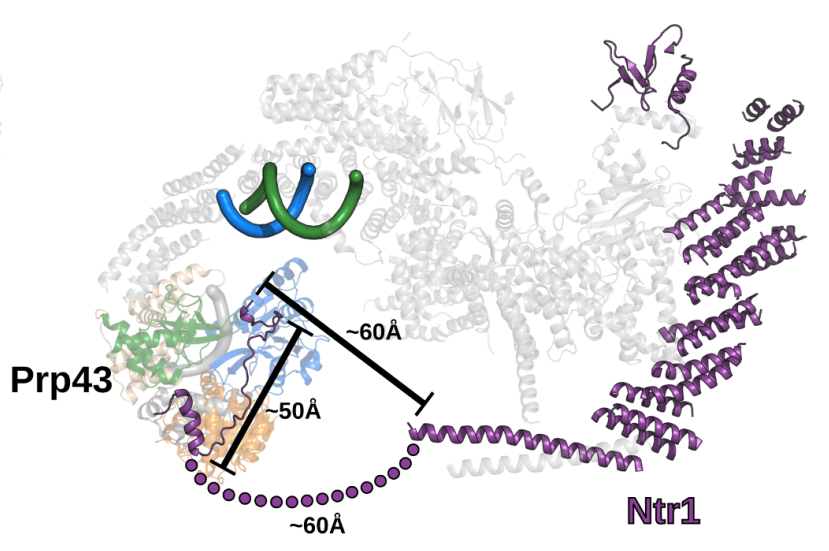

ILS

(PDBid: 5y88)

U2 SnRNA

U5 SnRNA

U6 SnRNA

pre-mRNA

Figure 15: Model of Ntr1 G-patch in the splicesosome. Protein components of the ILS (intron lariat spliceosome) are shown as cartoon models. snRNAs are depicted as tube models. The Ntr1 G-patch is modeled onto Prp43 based on a superposition of the Prp2-Spp2 G-patch complex structure on Prp43. (a) The bound G-patch is oriented towards the superhelical region of Ntr1. (b) The distances are given that need to be bridged between the cryo-EM Ntr1 model and the superposition-based G-patch model.

towards the superhelical region of Ntr1 (Fig. 15a). Interestingly, the modeled superhelical region of Ntr1 already contains a portion of the C-terminal section of the G-patch, which in the modeled G-patch based on the Prp2-Spp2 crystal structure is attached to the RecA2 domain. This leads to a discrepancy in location of about $60 \AA$ (Fig. 15b). Since the distance from the N-terminal helix of the G-patch to either the C-terminal end bound to RecA2 domain or to the superhelical region is about 50-60 $\AA$, it could be feasible that only the N-terminal helix binds to Prp43 in the spliceosomal context. On the other hand, due to the high dynamics of the spliceosomal peripheries, it could also be possible that the C-terminal G-patch region binds only transiently. Such a transient binding of this stretch would be in agreement with its alternative conformations found in the Prp2-Spp2 G-patch crystal structures (chapter 3).

Although the structural work on the Prp2-Spp2 G-patch complex could provide insights into how G-patch motifs are able to conformationally adapt to the DEAH-box ATPase domain movements during translocation, the structures only provide limited evidence on how stimulation is achieved (chapter 3). Since the G-patch does not directly contact adenosine nucleotide- or RNA-binding sites, it has been proposed that instead it has an impact on the dynamics of the ATPases. The C-terminal part of the G-patch binds to the RecA2 domain and could thereby influence its mobility that has been described to be vital for translocation (chapter 5). The $\mathrm{N}$-terminal $\alpha$-helix binds to the winged-helix domain, which is the hinge domain for the opening of the C- 


\section{Chapter 6: Discussion and Outlook}

terminal domains and due to this could have an impact on RNA loading (Tauchert et al., 2017). In fact, in the presence of Ntr1 Prp43 binds ssRNA with an about 100-fold higher affinity (Marieke Enders, personal communication). An additional factor could be that, once the RNA is loaded, Ntr1 locks the hinge function of the winged-helix domain and thereby hinders the release of the ssRNA out of the tunnel. This could explain the increased affinity towards ssRNA and the stimulation of translocation/unwinding. Furthermore, it could be shown that the release of bound ADP is favored in the presence of Ntr1 (Marieke Enders, personal communication). Such a function as nucleotide-exchange factor is in line with the stimulation of the ATPase

activity of Prp43 (Christian et al., 2014; Tauchert et al., 2017). These findings only start to deepen the knowledge about the interplay of DEAH-box ATPases with their G-patch proteins and extensive biophysical studies are still needed to fully understand the structural data obtained during this thesis. 


\section{Chapter 7: Abstract}

DEAH-box ATPases play a vital role in the activation, catalytic and disassembly steps of the spliceosome. They are key for the orchestration of conformational and compositional rearrangements during the splicing cycle. It has long been proposed that they do this by targeting double-stranded RNA networks and unwinding duplexes. However, recent structural as well as biochemical studies suggest that they rather act on singlestranded RNAs at the spliceosomal peripheries and thereby remotely remodel the spliceosome. While mainly structural approaches have tried to unravel the translocation mechanism of spliceosomal DEAH-box ATPases, only initial insights into the conformational dynamics of this family could be gained. It has been proven that the analysis of different ligand-bound states has been key to unveil domain movements and adenosine nucleotide-free states of spliceosomal DEAH-box ATPases still remained elusive at the beginning of this thesis. The determination of the apo structure of the Chaetomium thermophilum Prp22 revealed an intrinsic mobility of the RecA2 domain that allows open conformations of the helicase core previously undescribed. A bound RNA to the adenosine nucleotide-free Prp22 stabilizes the RecA2 domain into a defined open conformation of the helicase core. Compared to the RNA- and ATP-bound state of Prp43 with a closed helicase conformation, the adenosine nucleotide-free state is able to accommodate an additional RNA nucleotide in the binding tunnel. A continuous toggling between closed and open conformations of the helicase core, enables DEAH-box ATPases to translocate along an ssRNA with a step-size of one RNA nucleotide per hydrolyzed ATP. In order to synchronize these domain movements, a serine in the conserved sequence motif $\mathrm{V}$ is able to sense the catalytic state and accordingly position the RecA2 domain.

A further goal of this thesis was to study the discrepant way of functioning of Prp2. Prp2 plays a special role among spliceosomal DEAH-box ATPases as it is the only one not showing any in vitro unwinding capability. Comparing the $\mathrm{ADP}-\mathrm{BeF}_{3}-$ and RNA-bound structure of Chaetomium thermophilum Prp2 with a Prp43 structure in the same catalytic state, a loop in the C-terminal domains could be identified that ensures a divergent way of interaction with the RNA in Prp2. An insertion in this loop in Prp2 threads the RNA differently through the binding tunnel and might play a role in impeding Prp2 from being an unwindase. Additionally, complex structures of a Prp2-Spp2 G-patch complex could be solved and provide the first glimpse of a G-patch domain bound to its target protein. The structures show that the N-terminal part of the G-patch stably binds the winged-helix domain of Prp2, while the C-terminal end binds the RecA2 domain with two alternative conformations. Together with a flexible linker connecting these two parts, the G-patch exhibits an increased conformational flexibility that enables it to adapt to the movements of the RecA2 domain during translocation. 


\section{References}

Abelson, J., Trotta, C.R., and Li, H. (1998). tRNA Splicing. J. Biol. Chem. 273, 12685-12688.

Abovich, N., and Rosbash, M. (1997). Cross-Intron Bridging Interactions in the Yeast Commitment Complex Are Conserved in Mammals. Cell 89, 403-412.

Adams, P.D., Afonine, P.V., Bunkoczi, G., Chen, V.B., Davis, I.W., Echols, N., Headd, J.J., Hung, L.-W., Kapral, G.J., Grosse-Kunstleve, R.W., et al. (2010). PHENIX: a comprehensive Python-based system for macromolecular structure solution. Acta Crystallogr. Sect. D-Biol. Crystallogr. 66, 213-221.

Agafonov, D.E., Kastner, B., Dybkov, O., Hofele, R.V., Liu, W.-T., Urlaub, H., Lührmann, R., and Stark, H. (2016). Molecular architecture of the human U4/U6.U5 tri-snRNP. Science 351, 1416-1420.

Andersen, C.B.F., Ballut, L., Johansen, J.S., Chamieh, H., Nielsen, K.H., Oliveira, C.L.P., Pedersen, J.S., Séraphin, B., Le Hir, H., and Andersen, G.R. (2006). Structure of the exon junction core complex with a trapped DEAD-box ATPase bound to RNA. Science 313, 1968-1972.

Appleby, T.C., Anderson, R., Fedorova, O., Pyle, A.M., Wang, R., Liu, X., Brendza, K.M., and Somoza, J.R. (2011). Visualizing ATP-dependent RNA translocation by the NS3 helicase from HCV. J. Mol. Biol. 405, 1139-1153.

Aravind, L., and Koonin, E.V. (1999). G-patch: a new conserved domain in eukaryotic RNA-processing proteins and type D retroviral polyproteins. Trends Biochem. Sci. 24, 342-344.

Arenas, J.E., and Abelson, J.N. (1997). Prp43: An RNA helicase-like factor involved in spliceosome disassembly. Proc. Natl. Acad. Sci. U.S.A. 94, 11798-11802.

Aronova, A., Bacíková, D., Crotti, L.B., Horowitz, D.S., and Schwer, B. (2007). Functional interactions between Prp8, Prp18, Slu7, and U5 snRNA during the second step of pre-mRNA splicing. RNA 13, 14371444.

Bai, R., Yan, C., Wan, R., Lei, J., and Shi, Y. (2017). Structure of the Post-catalytic Spliceosome from Saccharomyces cerevisiae. Cell 171, 1589-1598.e8.

Bai, R., Wan, R., Yan, C., Lei, J., and Shi, Y. (2018). Structures of the fully assembled Saccharomyces cerevisiae spliceosome before activation. Science 360, 1423-1429.

Bao, P., Höbartner, C., Hartmuth, K., and Lührmann, R. (2017). Yeast Prp2 liberates the 5' splice site and the branch site adenosine for catalysis of pre-mRNA splicing. RNA 23, 1770-1779.

Berglund, J.A., Chua, K., Abovich, N., Reed, R., and Rosbash, M. (1997). The Splicing Factor BBP Interacts Specifically with the Pre-mRNA Branchpoint Sequence UACUAAC. Cell 89, 781-787.

Bertram, K., Agafonov, D.E., Liu, W.-T., Dybkov, O., Will, C.L., Hartmuth, K., Urlaub, H., Kastner, B., Stark, H., and Lührmann, R. (2017a). Cryo-EM structure of a human spliceosome activated for step 2 of splicing. Nature 542, 318-323. 


\section{References}

Bertram, K., Agafonov, D.E., Dybkov, O., Haselbach, D., Leelaram, M.N., Will, C.L., Urlaub, H., Kastner, B., Lührmann, R., and Stark, H. (2017b). Cryo-EM Structure of a Pre-catalytic Human Spliceosome Primed for Activation. Cell 170, 701-713.e11.

Bizebard, T., Ferlenghi, I., Iost, I., and Dreyfus, M. (2004). Studies on three E. coli DEAD-box helicases point to an unwinding mechanism different from that of model DNA helicases. Biochemistry 43, 7857-7866.

Bleichert, F., and Baserga, S.J. (2007). The long unwinding road of RNA helicases. Mol. Cell 27, 339-352.

Boesler, C., Rigo, N., Anokhina, M.M., Tauchert, M.J., Agafonov, D.E., Kastner, B., Urlaub, H., Ficner, R., Will, C.L., and Lührmann, R. (2016). A spliceosome intermediate with loosely associated tri-snRNP accumulates in the absence of Prp28 ATPase activity. Nature Communications 7, 11997.

Boneberg, F., Brandmann, T., Kobel, L., van den Heuvel, J., Bargsten, K., Bammert, L., Kutay, U., and Jinek, M. (2019). Molecular mechanism of the RNA helicase DHX37 and its activation by UTP14 in ribosome biogenesis. RNA.

Burgess, S.M., and Guthrie, C. (1993). A mechanism to enhance mRNA splicing fidelity: the RNAdependent ATPase Prp16 governs usage of a discard pathway for aberrant lariat intermediates. Cell 73, 13771391.

Büttner, K., Nehring, S., and Hopfner, K.-P. (2007). Structural basis for DNA duplex separation by a superfamily-2 helicase. Nature Structural \& Molecular Biology 14, 647-652.

Campodonico, E., and Schwer, B. (2002). ATP-Dependent Remodeling of the Spliceosome: Intragenic Suppressors of Release-Defective Mutants of Saccharomyces cerevisiae Prp22. Genetics 160, 407-415.

Caruthers, J.M., Johnson, E.R., and McKay, D.B. (2000). Crystal structure of yeast initiation factor 4A, a DEAD-box RNA helicase. Proc. Natl. Acad. Sci. U.S.A. 97, 13080-13085.

Cech, T.R. (1990). Self-splicing of group I introns. Annu. Rev. Biochem. 59, 543-568.

Charenton, C., Wilkinson, M.E., and Nagai, K. (2019). Mechanism of 5' splice site transfer for human spliceosome activation. Science 364, 362-367.

Chen, J.Y., Stands, L., Staley, J.P., Jackups, R.R., Latus, L.J., and Chang, T.H. (2001). Specific alterations of U1-C protein or U1 small nuclear RNA can eliminate the requirement of Prp28p, an essential DEAD box splicing factor. Mol. Cell 7, 227-232.

Chen, M.C., Tippana, R., Demeshkina, N.A., Murat, P., Balasubramanian, S., Myong, S., and FerréD'Amaré, A.R. (2018a). Structural basis of G-quadruplex unfolding by the DEAH/RHA helicase DHX36. Nature 558, 465.

Chen, V.B., Arendall, W.B., Headd, J.J., Keedy, D.A., Immormino, R.M., Kapral, G.J., Murray, L.W., Richardson, J.S., and Richardson, D.C. (2010). MolProbity: all-atom structure validation for macromolecular crystallography. Acta Crystallogr. D Biol. Crystallogr. 66, 12-21.

Chen, W.-F., Rety, S., Guo, H.-L., Dai, Y.-X., Wu, W.-Q., Liu, N.-N., Auguin, D., Liu, Q.-W., Hou, X.-M., Dou, S.-X., et al. (2018b). Molecular Mechanistic Insights into Drosophila DHX36-Mediated G-Quadruplex Unfolding: A Structure-Based Model. Structure 26, 403-415.e4. 


\section{References}

Cheng, Z., Coller, J., Parker, R., and Song, H. (2005). Crystal structure and functional analysis of DEAD-box protein Dhh1p. RNA 11, 1258-1270.

Chiu, Y.-F., Liu, Y.-C., Chiang, T.-W., Yeh, T.-C., Tseng, C.-K., Wu, N.-Y., and Cheng, S.-C. (2009). Cwc25 Is a Novel Splicing Factor Required after Prp2 and Yju2 To Facilitate the First Catalytic Reaction. Molecular and Cellular Biology 29, 5671-5678.

Christian, H., Hofele, R.V., Urlaub, H., and Ficner, R. (2014). Insights into the activation of the helicase Prp43 by biochemical studies and structural mass spectrometry. Nucleic Acids Res. 42, 1162-1179.

Company, M., Arenas, J., and Abelson, J. (1991). Requirement of the RNA helicase-like protein PRP22 for release of messenger RNA from spliceosomes. Nature 349, 487-493.

Cordin, O., and Beggs, J.D. (2013). RNA helicases in splicing. RNA Biol 10, 83-95.

Cordin, O., Hahn, D., and Beggs, J.D. (2012). Structure, function and regulation of spliceosomal RNA helicases. Curr. Opin. Cell Biol. 24, 431-438.

Crick, F. (1970). Central dogma of molecular biology. Nature 227, 561-563.

Darzacq, X., Jády, B.E., Verheggen, C., Kiss, A.M., Bertrand, E., and Kiss, T. (2002). Cajal body-specific small nuclear RNAs: a novel class of 2'-O-methylation and pseudouridylation guide RNAs. EMBO J. 21, 2746-2756.

Del Campo, M., and Lambowitz, A.M. (2009). Structure of the Yeast DEAD box protein Mss116p reveals two wedges that crimp RNA. Mol. Cell 35, 598-609.

Devarkar, S.C., Schweibenz, B., Wang, C., Marcotrigiano, J., and Patel, S.S. (2018). RIG-I Uses an ATPasePowered Translocation-Throttling Mechanism for Kinetic Proofreading of RNAs and Oligomerization. Mol. Cell 72, 355-368.e4.

Ding, S.C., and Pyle, A.M. (2012). Molecular mechanics of RNA translocases. Meth. Enzymol. 511, 131147.

Dittrich, M., and Schulten, K. (2005). Zooming in on ATP hydrolysis in F1. J. Bioenerg. Biomembr. 37, 441444.

Drummond, D.R., Armstrong, J., and Colman, A. (1985). The effect of capping and polyadenylation on the stability, movement and translation of synthetic messenger RNAs in Xenopus oocytes. Nucleic Acids Res. 13, 7375-7394.

Fabrizio, P., Dannenberg, J., Dube, P., Kastner, B., Stark, H., Urlaub, H., and Lührmann, R. (2009). The evolutionarily conserved core design of the catalytic activation step of the yeast spliceosome. Mol. Cell 36, 593-608.

Fairman-Williams, M.E., Guenther, U.-P., and Jankowsky, E. (2010). SF1 and SF2 helicases: family matters. Curr. Opin. Struct. Biol. 20, 313-324.

Fica, S.M., Tuttle, N., Novak, T., Li, N.-S., Lu, J., Koodathingal, P., Dai, Q., Staley, J.P., and Piccirilli, J.A. (2013). RNA catalyses nuclear pre-mRNA splicing. Nature 503, 229-234. 


\section{References}

Fica, S.M., Oubridge, C., Galej, W.P., Wilkinson, M.E., Bai, X.-C., Newman, A.J., and Nagai, K. (2017). Structure of a spliceosome remodelled for exon ligation. Nature 542, 377-380.

Fica, S.M., Oubridge, C., Wilkinson, M.E., Newman, A.J., and Nagai, K. (2019). A human postcatalytic spliceosome structure reveals essential roles of metazoan factors for exon ligation. Science 363, 710-714.

Fouraux, M.A., Kolkman, M.J.M., Van der Heijden, A., De Jong, A.S., Van Venrooij, W.J., and Pruijn, G.J.M. (2002). The human La (SS-B) autoantigen interacts with DDX15/hPrp43, a putative DEAH-box RNA helicase. RNA 8, 1428-1443.

Fourmann, J.-B., Dybkov, O., Agafonov, D.E., Tauchert, M.J., Urlaub, H., Ficner, R., Fabrizio, P., and Lührmann, R. (2016). The target of the DEAH-box NTP triphosphatase Prp43 in Saccharomyces cerevisiae spliceosomes is the U2 snRNP-intron interaction. ELife 5, e15564.

Galej, W.P. (2018). Structural studies of the spliceosome: past, present and future perspectives. Biochem. Soc. Trans.

Galej, W.P., Wilkinson, M.E., Fica, S.M., Oubridge, C., Newman, A.J., and Nagai, K. (2016). Cryo-EM structure of the spliceosome immediately after branching. Nature 537, 197-201.

Goldfeder, M.B., and Oliveira, C.C. (2008). Cwc24p, a novel Saccharomyces cerevisiae nuclear ring finger protein, affects pre-snoRNA U3 splicing. J. Biol. Chem. 283, 2644-2653.

Gozani, O., Potashkin, J., and Reed, R. (1998). A potential role for U2AF-SAP 155 interactions in recruiting U2 snRNP to the branch site. Mol. Cell. Biol. 18, 4752-4760.

Gross, C.H., and Shuman, S. (1998). The nucleoside triphosphatase and helicase activities of vaccinia virus NPH-II are essential for virus replication. J. Virol. 72, 4729-4736.

Gu, M., and Rice, C.M. (2010). Three conformational snapshots of the hepatitis C virus NS3 helicase reveal a ratchet translocation mechanism. Proc. Natl. Acad. Sci. U.S.A. 107, 521-528.

Guglielmi, B., and Werner, M. (2002). The yeast homolog of human PinX1 is involved in rRNA and small nucleolar RNA maturation, not in telomere elongation inhibition. J. Biol. Chem. 277, 35712-35719.

Hamann, F., Enders, M., and Ficner, R. (2019). Structural basis for RNA translocation by DEAH-box ATPases. Nucleic Acids Res.

Hang, J., Wan, R., Yan, C., and Shi, Y. (2015). Structural basis of pre-mRNA splicing. Science 349, 11911198.

Haselbach, D., Komarov, I., Agafonov, D.E., Hartmuth, K., Graf, B., Dybkov, O., Urlaub, H., Kastner, B., Lührmann, R., and Stark, H. (2018). Structure and Conformational Dynamics of the Human Spliceosomal Bact Complex. Cell 172, 454-464.e11.

He, Y., Andersen, G.R., and Nielsen, K.H. (2010). Structural basis for the function of DEAH helicases. EMBO Rep 11, 180-186.

He, Y., Staley, J.P., Andersen, G.R., and Nielsen, K.H. (2017). Structure of the DEAH/RHA ATPase Prp43p bound to RNA implicates a pair of hairpins and motif Va in translocation along RNA. RNA 23, 1110-1124. 


\section{References}

Heilek, G.M., and Peterson, M.G. (1997). A point mutation abolishes the helicase but not the nucleoside triphosphatase activity of hepatitis C virus NS3 protein. J. Virol. 71, 6264-6266.

Heininger, A.U., Hackert, P., Andreou, A.Z., Boon, K.-L., Memet, I., Prior, M., Clancy, A., Schmidt, B., Urlaub, H., Schleiff, E., et al. (2016). Protein cofactor competition regulates the action of a multifunctional RNA helicase in different pathways. RNA Biol 13, 320-330.

Hu, W.-S., and Hughes, S.H. (2012). HIV-1 reverse transcription. Cold Spring Harb Perspect Med 2.

Iyer, L.M., Leipe, D.D., Koonin, E.V., and Aravind, L. (2004). Evolutionary history and higher order classification of AAA+ ATPases. J. Struct. Biol. 146, 11-31.

Jacquier, A. (1990). Self-splicing group II and nuclear pre-mRNA introns: how similar are they? Trends Biochem. Sci. 15, 351-354.

Jády, B.E., Darzacq, X., Tucker, K.E., Matera, A.G., Bertrand, E., and Kiss, T. (2003). Modification of Sm small nuclear RNAs occurs in the nucleoplasmic Cajal body following import from the cytoplasm. EMBO J. 22, 1878-1888.

James, S.-A., Turner, W., and Schwer, B. (2002). How Slu7 and Prp18 cooperate in the second step of yeast pre-mRNA splicing. RNA 8, 1068-1077.

Jiang, F., Ramanathan, A., Miller, M.T., Tang, G.-Q., Gale, M., Patel, S.S., and Marcotrigiano, J. (2011). Structural basis of RNA recognition and activation by innate immune receptor RIG-I. Nature 479, 423-427.

Kabsch, W. (2010). XDS. Acta Crystallogr. D Biol. Crystallogr. 66, 125-132.

Kim, S.H., and Lin, R.J. (1996). Spliceosome activation by PRP2 ATPase prior to the first transesterification reaction of pre-mRNA splicing. Mol. Cell. Biol. 16, 6810-6819.

Kim, S.H., Smith, J., Claude, A., and Lin, R.J. (1992). The purified yeast pre-mRNA splicing factor PRP2 is an RNA-dependent NTPase. EMBO J. 11, 2319-2326.

King, D.S., and Beggs, J.D. (1990). Interactions of PRP2 protein with pre-mRNA splicing complexes in Saccharomyces cerevisiae. Nucleic Acids Res. 18, 6559-6564.

Kistler, A.L., and Guthrie, C. (2001). Deletion of MUD2, the yeast homolog of U2AF65, can bypass the requirement for Sub2, an essential spliceosomal ATPase. Genes Dev. 15, 42-49.

Kleywegt, G.J. (1996). Use of Non-crystallographic Symmetry in Protein Structure Refinement. Acta Cryst D 52, 842-857.

Konarska, M.M., Vilardell, J., and Query, C.C. (2006). Repositioning of the Reaction Intermediate within the Catalytic Center of the Spliceosome. Molecular Cell 21, 543-553.

Krishnan, R., Blanco, M.R., Kahlscheuer, M.L., Abelson, J., Guthrie, C., and Walter, N.G. (2013). Biased Brownian ratcheting leads to pre-mRNA remodeling and capture prior to first-step splicing. Nature Structural \& Molecular Biology 20, 1450-1457.

Kruger, K., Grabowski, P.J., Zaug, A.J., Sands, J., Gottschling, D.E., and Cech, T.R. (1982). Self-splicing RNA: autoexcision and autocyclization of the ribosomal RNA intervening sequence of Tetrahymena. Cell 31, 147-157. 


\section{References}

Kull, F.J., and Endow, S.A. (2002). Kinesin: switch I \& II and the motor mechanism. J. Cell. Sci. 115, 15-23.

Laggerbauer, B., Achsel, T., and Lührmann, R. (1998). The human U5-200kD DEXH-box protein unwinds U4/U6 RNA duplices in vitro. PNAS 95, 4188-4192.

Lange, T.S., and Gerbi, S.A. (2000). Transient nucleolar localization Of U6 small nuclear RNA in Xenopus Laevis oocytes. Mol. Biol. Cell 11, 2419-2428.

Lardelli, R.M., Thompson, J.X., Yates, J.R., and Stevens, S.W. (2010). Release of SF3 from the intron branchpoint activates the first step of pre-mRNA splicing. RNA 16, 516-528.

Lebaron, S., Papin, C., Capeyrou, R., Chen, Y.-L., Froment, C., Monsarrat, B., Caizergues-Ferrer, M., Grigoriev, M., and Henry, Y. (2009). The ATPase and helicase activities of Prp43p are stimulated by the Gpatch protein Pfa1p during yeast ribosome biogenesis. EMBO J. 28, 3808-3819.

Leitão, A.L., Costa, M.C., and Enguita, F.J. (2015). Unzippers, resolvers and sensors: a structural and functional biochemistry tale of RNA helicases. Int J Mol Sci 16, 2269-2293.

Li, X., Liu, S., Jiang, J., Zhang, L., Espinosa, S., Hill, R.C., Hansen, K.C., Zhou, Z.H., and Zhao, R. (2017). CryoEM structure of Saccharomyces cerevisiae U1 snRNP offers insight into alternative splicing. Nat Commun 8, 1035.

Liu, H.-L., and Cheng, S.-C. (2012). The interaction of Prp2 with a defined region of the intron is required for the first splicing reaction. Mol. Cell. Biol. 32, 5056-5066.

Liu, S., Li, X., Zhang, L., Jiang, J., Hill, R.C., Cui, Y., Hansen, K.C., Zhou, Z.H., and Zhao, R. (2017). Structure of the yeast spliceosomal postcatalytic P complex. Science 358, 1278-1283.

Liu, Y.-C., Chen, H.-C., Wu, N.-Y., and Cheng, S.-C. (2007). A novel splicing factor, Yju2, is associated with NTC and acts after Prp2 in promoting the first catalytic reaction of pre-mRNA splicing. Mol. Cell. Biol. 27, 5403-5413.

Lohmann, V. (2013). Hepatitis C virus RNA replication. Curr. Top. Microbiol. Immunol. 369, 167-198.

Luo, D., Ding, S.C., Vela, A., Kohlway, A., Lindenbach, B.D., and Pyle, A.M. (2011). Structural insights into RNA recognition by RIG-I. Cell 147, 409-422.

Madhani, H.D., and Guthrie, C. (1992). A novel base-pairing interaction between U2 and U6 snRNAs suggests a mechanism for the catalytic activation of the spliceosome. Cell 71, 803-817.

Martin, A., Schneider, S., and Schwer, B. (2002). Prp43 is an essential RNA-dependent ATPase required for release of lariat-intron from the spliceosome. J. Biol. Chem. 277, 17743-17750.

Matera, A.G., and Wang, Z. (2014). A day in the life of the spliceosome. Nat. Rev. Mol. Cell Biol. 15, 108121.

Mattaj, I.W. (1986). Cap trimethylation of U snRNA is cytoplasmic and dependent on U snRNP protein binding. Cell 46, 905-911.

Mayas, R.M., Maita, H., and Staley, J.P. (2006). Exon ligation is proofread by the DExD/H-box ATPase Prp22p. Nat. Struct. Mol. Biol. 13, 482-490. 


\section{References}

McCoy, A.J., Grosse-Kunstleve, R.W., Adams, P.D., Winn, M.D., Storoni, L.C., and Read, R.J. (2007). Phaser crystallographic software. J Appl Crystallogr 40, 658-674.

Milburn, M.V., Tong, L., deVos, A.M., Brünger, A., Yamaizumi, Z., Nishimura, S., and Kim, S.H. (1990). Molecular switch for signal transduction: structural differences between active and inactive forms of protooncogenic ras proteins. Science 247, 939-945.

Myong, S., Bruno, M.M., Pyle, A.M., and Ha, T. (2007). Spring-loaded mechanism of DNA unwinding by hepatitis C virus NS3 helicase. Science 317, 513-516.

Narayanan, U., Achsel, T., Lührmann, R., and Matera, A.G. (2004). Coupled in vitro import of U snRNPs and SMN, the spinal muscular atrophy protein. Mol. Cell 16, 223-234.

Nguyen, T.H.D., Galej, W.P., Bai, X., Savva, C.G., Newman, A.J., Scheres, S.H.W., and Nagai, K. (2015). The architecture of the spliceosomal U4/U6.U5 tri-snRNP. Nature 523, 47-52.

Nguyen, T.H.D., Galej, W.P., Bai, X.-C., Oubridge, C., Newman, A.J., Scheres, S.H.W., and Nagai, K. (2016). Cryo-EM structure of the yeast U4/U6.U5 tri-snRNP at $3.7 \AA$ resolution. Nature 530, 298-302.

Nilsen, T.W., and Graveley, B.R. (2010). Expansion of the eukaryotic proteome by alternative splicing. Nature 463, 457-463.

van Nues, R.W., and Beggs, J.D. (2001). Functional contacts with a range of splicing proteins suggest a central role for Brr2p in the dynamic control of the order of events in spliceosomes of Saccharomyces cerevisiae. Genetics 157, 1451-1467.

Ohrt, T., Prior, M., Dannenberg, J., Odenwälder, P., Dybkov, O., Rasche, N., Schmitzová, J., Gregor, I., Fabrizio, P., Enderlein, J., et al. (2012). Prp2-mediated protein rearrangements at the catalytic core of the spliceosome as revealed by dcFCCS. RNA 18, 1244-1256.

Ohrt, T., Odenwälder, P., Dannenberg, J., Prior, M., Warkocki, Z., Schmitzová, J., Karaduman, R., Gregor, I., Enderlein, J., Fabrizio, P., et al. (2013). Molecular dissection of step 2 catalysis of yeast pre-mRNA splicing investigated in a purified system. RNA 19, 902-915.

Ozgur, S., Buchwald, G., Falk, S., Chakrabarti, S., Prabu, J.R., and Conti, E. (2015). The conformational plasticity of eukaryotic RNA-dependent ATPases. FEBS J. 282, 850-863.

Paule, M.R., and White, R.J. (2000). Survey and summary: transcription by RNA polymerases I and III. Nucleic Acids Res. 28, 1283-1298.

Pause, A., and Sonenberg, N. (1992). Mutational analysis of a DEAD box RNA helicase: the mammalian translation initiation factor eIF-4A. EMBO J. 11, 2643-2654.

Peck, M.L., and Herschlag, D. (1999). Effects of oligonucleotide length and atomic composition on stimulation of the ATPase activity of translation initiation factor elF4A. RNA 5, 1210-1221.

Peebles, C.L., Perlman, P.S., Mecklenburg, K.L., Petrillo, M.L., Tabor, J.H., Jarrell, K.A., and Cheng, H.L. (1986). A self-splicing RNA excises an intron lariat. Cell 44, 213-223.

Plaschka, C., Lin, P.-C., and Nagai, K. (2017). Structure of a pre-catalytic spliceosome. Nature 546, 617621. 


\section{References}

Plaschka, C., Lin, P.-C., Charenton, C., and Nagai, K. (2018). Prespliceosome structure provides insights into spliceosome assembly and regulation. Nature 559, 419-422.

Plessel, G., Lührmann, R., and Kastner, B. (1997). Electron microscopy of assembly intermediates of the snRNP core: morphological similarities between the RNA-free (E.F.G) protein heteromer and the intact snRNP core. J. Mol. Biol. 265, 87-94.

Prabu, J.R., Müller, M., Thomae, A.W., Schüssler, S., Bonneau, F., Becker, P.B., and Conti, E. (2015). Structure of the RNA Helicase MLE Reveals the Molecular Mechanisms for Uridine Specificity and RNAATP Coupling. Molecular Cell 60, 487-499.

Pyle, A.M. (2008). Translocation and unwinding mechanisms of RNA and DNA helicases. Annu Rev Biophys 37, 317-336.

Raghunathan, P.L., and Guthrie, C. (1998a). RNA unwinding in U4/U6 snRNPs requires ATP hydrolysis and the DEIH-box splicing factor Brr2. Curr. Biol. 8, 847-855.

Raghunathan, P.L., and Guthrie, C. (1998b). A spliceosomal recycling factor that reanneals U4 and U6 small nuclear ribonucleoprotein particles. Science 279, 857-860.

Rauhut, R., Fabrizio, P., Dybkov, O., Hartmuth, K., Pena, V., Chari, A., Kumar, V., Lee, C.-T., Urlaub, H., Kastner, B., et al. (2016). Molecular architecture of the Saccharomyces cerevisiae activated spliceosome. Science 353, 1399-1405.

Robart, A.R., Chan, R.T., Peters, J.K., Rajashankar, K.R., and Toor, N. (2014). Crystal structure of a eukaryotic group II intron lariat. Nature 514, 193-197.

Robert-Paganin, J., Réty, S., and Leulliot, N. (2015). Regulation of DEAH/RHA helicases by G-patch proteins. Biomed Res Int 2015, 931857.

Rogers, G.W., Lima, W.F., and Merrick, W.C. (2001). Further characterization of the helicase activity of eIF4A. Substrate specificity. J. Biol. Chem. 276, 12598-12608.

Roy, J., Kim, K., Maddock, J.R., Anthony, J.G., and Woolford, J.L. (1995). The final stages of spliceosome maturation require Spp2p that can interact with the DEAH box protein Prp2p and promote step 1 of splicing. RNA 1, 375-390.

Roychowdhury, A., Joret, C., Bourgeois, G., Heurgué-Hamard, V., Lafontaine, D.L.J., and Graille, M. (2019). The DEAH-box RNA helicase Dhr1 contains a remarkable carboxyl terminal domain essential for small ribosomal subunit biogenesis. Nucleic Acids Res.

Saikrishnan, K., Powell, B., Cook, N.J., Webb, M.R., and Wigley, D.B. (2009). Mechanistic basis of 5'-3’ translocation in SF1B helicases. Cell 137, 849-859.

Santos, K.F., Jovin, S.M., Weber, G., Pena, V., Lührmann, R., and Wahl, M.C. (2012). Structural basis for functional cooperation between tandem helicase cassettes in Brr2-mediated remodeling of the spliceosome. Proc. Natl. Acad. Sci. U.S.A. 109, 17418-17423.

Schmitt, A., Hamann, F., Neumann, P., and Ficner, R. (2018). Crystal structure of the spliceosomal DEAHbox ATPase Prp2. Acta Crystallogr D Struct Biol 74, 643-654. 


\section{References}

Schneider, S., and Schwer, B. (2001). Functional domains of the yeast splicing factor Prp22p. J. Biol. Chem. 276, 21184-21191.

Schneider, S., Campodonico, E., and Schwer, B. (2004). Motifs IV and V in the DEAH box splicing factor Prp22 are important for RNA unwinding, and helicase-defective Prp22 mutants are suppressed by Prp8. J. Biol. Chem. 279, 8617-8626.

Schwer, B. (2001). A new twist on RNA helicases: DExH/D box proteins as RNPases. Nat. Struct. Biol. 8, 113-116.

Schwer, B. (2008). A conformational rearrangement in the spliceosome sets the stage for Prp22-dependent mRNA release. Mol. Cell 30, 743-754.

Schwer, B., and Gross, C.H. (1998). Prp22, a DExH-box RNA helicase, plays two distinct roles in yeast premRNA splicing. EMBO J 17, 2086-2094.

Schwer, B., and Guthrie, C. (1991). PRP16 is an RNA-dependent ATPase that interacts transiently with the spliceosome. Nature 349, 494-499.

Schwer, B., and Guthrie, C. (1992). A conformational rearrangement in the spliceosome is dependent on PRP16 and ATP hydrolysis. The EMBO Journal 11, 5033-5039.

Schwer, B., and Meszaros, T. (2000). RNA helicase dynamics in pre-mRNA splicing. EMBO J. 19, 65826591.

Semlow, D.R., Blanco, M.R., Walter, N.G., and Staley, J.P. (2016). Spliceosomal DEAH-Box ATPases Remodel Pre-mRNA to Activate Alternative Splice Sites. Cell 164, 985-998.

Sengoku, T., Nureki, O., Nakamura, A., Kobayashi, S., and Yokoyama, S. (2006). Structural basis for RNA unwinding by the DEAD-box protein Drosophila Vasa. Cell 125, 287-300.

Séraphin, B., Kretzner, L., and Rosbash, M. (1988). A U1 snRNA:pre-mRNA base pairing interaction is required early in yeast spliceosome assembly but does not uniquely define the $5^{\prime}$ cleavage site. The EMBO Journal 7, 2533-2538.

Shaw, D.J., Eggleton, P., and Young, P.J. (2008). Joining the dots: production, processing and targeting of U snRNP to nuclear bodies. Biochim. Biophys. Acta 1783, 2137-2144.

Siliciano, P.G., and Guthrie, C. (1988). 5' splice site selection in yeast: genetic alterations in base-pairing with U1 reveal additional requirements. Genes Dev. 2, 1258-1267.

Silverman, E.J., Maeda, A., Wei, J., Smith, P., Beggs, J.D., and Lin, R.-J. (2004). Interaction between a Gpatch protein and a spliceosomal DEXD/H-box ATPase that is critical for splicing. Mol. Cell. Biol. 24, 10101-10110.

Singh, R., and Reddy, R. (1989). Gamma-monomethyl phosphate: a cap structure in spliceosomal U6 small nuclear RNA. Proc. Natl. Acad. Sci. U.S.A. 86, 8280-8283.

Smith, D.J., Query, C.C., and Konarska, M.M. (2008). “Nought May Endure but Mutability”: Spliceosome Dynamics and the Regulation of Splicing. Molecular Cell 30, 657-666. 


\section{References}

Staley, J.P., and Guthrie, C. (1999). An RNA Switch at the 5' Splice Site Requires ATP and the DEAD Box Protein Prp28p. Molecular Cell 3, 55-64.

Steitz, T.A., and Steitz, J.A. (1993). A general two-metal-ion mechanism for catalytic RNA. Proc. Natl. Acad. Sci. U.S.A. 90, 6498-6502.

Tanaka, N., and Schwer, B. (2005). Characterization of the NTPase, RNA-Binding, and RNA Helicase Activities of the DEAH-Box Splicing Factor Prp22. Biochemistry 44, 9795-9803.

Tanaka, N., and Schwer, B. (2006). Mutations in PRP43 that uncouple RNA-dependent NTPase activity and pre-mRNA splicing function. Biochemistry 45, 6510-6521.

Tanaka, N., Aronova, A., and Schwer, B. (2007). Ntr1 activates the Prp43 helicase to trigger release of lariatintron from the spliceosome. Genes Dev. 21, 2312-2325.

Tanner, N.K., Cordin, O., Banroques, J., Doère, M., and Linder, P. (2003). The Q motif: a newly identified motif in DEAD box helicases may regulate ATP binding and hydrolysis. Mol. Cell 11, 127-138.

Tauchert, M.J., Fourmann, J.-B., Christian, H., Lührmann, R., and Ficner, R. (2016). Structural and functional analysis of the RNA helicase Prp43 from the thermophilic eukaryote Chaetomium thermophilum. Acta Crystallogr F Struct Biol Commun 72, 112-120.

Tauchert, M.J., Fourmann, J.-B., Lührmann, R., and Ficner, R. (2017). Structural insights into the mechanism of the DEAH-box RNA helicase Prp43. ELife 6, e21510.

Teigelkamp, S., McGarvey, M., Plumpton, M., and Beggs, J.D. (1994). The splicing factor PRP2, a putative RNA helicase, interacts directly with pre-mRNA. EMBO J. 13, 888-897.

Tsai, R.-T., Fu, R.-H., Yeh, F.-L., Tseng, C.-K., Lin, Y.-C., Huang, Y.-H., and Cheng, S.-C. (2005).

Spliceosome disassembly catalyzed by Prp43 and its associated components Ntr1 and Ntr2. Genes Dev. 19, 2991-3003.

Tsai, R.-T., Tseng, C.-K., Lee, P.-J., Chen, H.-C., Fu, R.-H., Chang, K., Yeh, F.-L., and Cheng, S.-C. (2007). Dynamic interactions of Ntr1-Ntr2 with Prp43 and with U5 govern the recruitment of Prp43 to mediate spliceosome disassembly. Mol. Cell. Biol. 27, 8027-8037.

Tseng, C.-K., Liu, H.-L., and Cheng, S.-C. (2011). DEAH-box ATPase Prp16 has dual roles in remodeling of the spliceosome in catalytic steps. RNA 17, 145-154.

Velankar, S.S., Soultanas, P., Dillingham, M.S., Subramanya, H.S., and Wigley, D.B. (1999). Crystal structures of complexes of PcrA DNA helicase with a DNA substrate indicate an inchworm mechanism. Cell $97,75-84$.

Vetter, I.R., and Wittinghofer, A. (2001). The guanine nucleotide-binding switch in three dimensions. Science 294, 1299-1304.

Wagner, J.D.O., Jankowsky, E., Company, M., Pyle, A.M., and Abelson, J.N. (1998). The DEAH-box protein PRP22 is an ATPase that mediates ATP-dependent mRNA release from the spliceosome and unwinds RNA duplexes. The EMBO Journal 17, 2926-2937.

Wahl, M.C., Will, C.L., and Lührmann, R. (2009). The spliceosome: design principles of a dynamic RNP machine. Cell 136, 701-718. 


\section{References}

Walbott, H., Mouffok, S., Capeyrou, R., Lebaron, S., Humbert, O., van Tilbeurgh, H., Henry, Y., and Leulliot, N. (2010). Prp43p contains a processive helicase structural architecture with a specific regulatory domain. EMBO J. 29, 2194-2204.

Walker, J.E., Saraste, M., Runswick, M.J., and Gay, N.J. (1982). Distantly related sequences in the alphaand beta-subunits of ATP synthase, myosin, kinases and other ATP-requiring enzymes and a common nucleotide binding fold. EMBO J. 1, 945-951.

Wan, R., Yan, C., Bai, R., Wang, L., Huang, M., Wong, C.C.L., and Shi, Y. (2016a). The 3.8 A structure of the U4/U6.U5 tri-snRNP: Insights into spliceosome assembly and catalysis. Science 351, 466-475.

Wan, R., Yan, C., Bai, R., Huang, G., and Shi, Y. (2016b). Structure of a yeast catalytic step I spliceosome at 3.4 Å resolution. Science 353, 895-904.

Wan, R., Yan, C., Bai, R., Lei, J., and Shi, Y. (2017). Structure of an Intron Lariat Spliceosome from Saccharomyces cerevisiae. Cell 171, 120-132.e12.

Wan, R., Bai, R., Yan, C., Lei, J., and Shi, Y. (2019). Structures of the Catalytically Activated Yeast Spliceosome Reveal the Mechanism of Branching. Cell 177, 339-351.e13.

Wang, Y., and Guthrie, C. (1998). PRP16, a DEAH-box RNA helicase, is recruited to the spliceosome primarily via its nonconserved N-terminal domain. RNA 4, 1216-1229.

Wang, Y., Wagner, J.D., and Guthrie, C. (1998). The DEAH-box splicing factor Prp16 unwinds RNA duplexes in vitro. Curr. Biol. 8, 441-451.

Warkocki, Z., Odenwälder, P., Schmitzová, J., Platzmann, F., Stark, H., Urlaub, H., Ficner, R., Fabrizio, P., and Lührmann, R. (2009). Reconstitution of both steps of Saccharomyces cerevisiae splicing with purified spliceosomal components. Nat. Struct. Mol. Biol. 16, 1237-1243.

Warkocki, Z., Schneider, C., Mozaffari-Jovin, S., Schmitzová, J., Höbartner, C., Fabrizio, P., and Lührmann, R. (2015). The G-patch protein Spp2 couples the spliceosome-stimulated ATPase activity of the DEAH-box protein Prp2 to catalytic activation of the spliceosome. Genes Dev. 29, 94-107.

Weir, J.R., Bonneau, F., Hentschel, J., and Conti, E. (2010). Structural analysis reveals the characteristic features of Mtr4, a DExH helicase involved in nuclear RNA processing and surveillance. Proc. Natl. Acad. Sci. U.S.A. 107, 12139-12144.

Wilkinson, M.E., Fica, S.M., Galej, W.P., Norman, C.M., Newman, A.J., and Nagai, K. (2017). Postcatalytic spliceosome structure reveals mechanism of 3'-splice site selection. Science 358, 1283-1288.

Will, C.L., and Lührmann, R. (2011). Spliceosome structure and function. Cold Spring Harb Perspect Biol 3.

Wittinghofer, A., and Vetter, I.R. (2011). Structure-function relationships of the G domain, a canonical switch motif. Annu. Rev. Biochem. 80, 943-971.

Wong, E.V., Cao, W., Vörös, J., Merchant, M., Modis, Y., Hackney, D.D., Montpetit, B., and De La Cruz, E.M. (2016). P(I) Release Limits the Intrinsic and RNA-Stimulated ATPase Cycles of DEAD-Box Protein 5 (Dbp5). J. Mol. Biol. 428, 492-508.

Xu, Y.-Z., and Query, C.C. (2007). Competition between the ATPase Prp5 and Branch Region-U2 snRNA Pairing Modulates the Fidelity of Spliceosome Assembly. Molecular Cell 28, 838-849. 


\section{References}

Yan, C., Hang, J., Wan, R., Huang, M., Wong, C.C.L., and Shi, Y. (2015). Structure of a yeast spliceosome at 3.6-angstrom resolution. Science 349, 1182-1191.

Yan, C., Wan, R., Bai, R., Huang, G., and Shi, Y. (2016). Structure of a yeast activated spliceosome at $3.5 \AA$ resolution. Science 353, 904-911.

Yan, C., Wan, R., Bai, R., Huang, G., and Shi, Y. (2017). Structure of a yeast step II catalytically activated spliceosome. Science 355, 149-155.

Yang, Q., and Jankowsky, E. (2006). The DEAD-box protein Ded1 unwinds RNA duplexes by a mode distinct from translocating helicases. Nat. Struct. Mol. Biol. 13, 981-986.

Yean, S.L., and Lin, R.J. (1991). U4 small nuclear RNA dissociates from a yeast spliceosome and does not participate in the subsequent splicing reaction. Mol. Cell. Biol. 11, 5571-5577.

Zhan, X., Yan, C., Zhang, X., Lei, J., and Shi, Y. (2018a). Structures of the human pre-catalytic spliceosome and its precursor spliceosome. Cell Res. 28, 1129-1140.

Zhan, X., Yan, C., Zhang, X., Lei, J., and Shi, Y. (2018b). Structure of a human catalytic step I spliceosome. Science 359, 537-545.

Zhang, X., Yan, C., Hang, J., Finci, L.I., Lei, J., and Shi, Y. (2017). An Atomic Structure of the Human Spliceosome. Cell 169, 918-929.e14.

Zhang, X., Yan, C., Zhan, X., Li, L., Lei, J., and Shi, Y. (2018). Structure of the human activated spliceosome in three conformational states. Cell Res. 28, 307-322.

Zhang, X., Zhan, X., Yan, C., Zhang, W., Liu, D., Lei, J., and Shi, Y. (2019). Structures of the human spliceosomes before and after release of the ligated exon. Cell Res.

Zhang, Z.-M., Yang, F., Zhang, J., Tang, Q., Li, J., Gu, J., Zhou, J., and Xu, Y.-Z. (2013). Crystal structure of Prp5p reveals interdomain interactions that impact spliceosome assembly. Cell Rep 5, 1269-1278.

Zhuang, Y., and Weiner, A.M. (1986). A compensatory base change in U1 snRNA suppresses a 5' splice site mutation. Cell 46, 827-835. 
Abbreviations

\section{Abbreviations}

\begin{tabular}{|c|c|}
\hline${ }^{\circ} \mathrm{C}$ & Degree Celsius \\
\hline $2 ’ \mathrm{OH}$ & 2‘ hyroxy \\
\hline 3’sS & 3’ splice site \\
\hline 3’OH & 3‘ hydroxy \\
\hline 5'sS & 5’ splice site \\
\hline 6-FAM & 6-Carboxyfluorescein \\
\hline A & Adenine \\
\hline$\AA$ & Angström \\
\hline $\mathrm{AAA}^{+}$ & ATPase associated with diverse cellular activities \\
\hline ADP & Adenosine diphosphate \\
\hline AMPPNP & Adenosine-5'-[( $\beta, \gamma)$-imido]triphosphate \\
\hline ATP & Adenosine triphosphate \\
\hline $\mathrm{B}^{\text {act }}$ & Activated complex B \\
\hline Brr2 & Bad response to refrigeration 2 \\
\hline $\mathrm{CD}$ & Circular dichroism \\
\hline CDP & Cytidine diphosphate \\
\hline Cmg1 & Cytoplasmic and mitochondrial G-patch protein 1 \\
\hline Cryo-EM & Cryo-electron microscopy \\
\hline $\mathrm{ct}$ & Chaetomium thermophilum \\
\hline C. thermophilum & Chaetomium thermophilum \\
\hline C-terminal & Carboxy-terminal \\
\hline Cus1 & Cold sensitive U2 snRNA Suppressor 1 \\
\hline Cwc & Complexed with CEF1 \\
\hline
\end{tabular}




\section{Abbreviations}

Da

Dbp5

dcFCCS

DEAD

DEAH

Desy

DFG

DNA

DHX

Dib1

E. coli

eIF4A

ESRF

Fn

Gno1

G-patch

GTP

HB

$\mathrm{HCV}$

Hel308

HEPES

hs

H. sapiens

Hsh155

ILS

ISL
Dalton

DEAD box protein 5

Dual-color fluorescence cross-correlation spectroscopy

aspartate-glutamate-alanine-aspartate

aspartate-glutamate-alanine-histidine

Deutsches Elektronen-Synchrotron

Deutsche Forschungsgemeinschaft

deoxyribonucleic acid

DEAH-box helicase

DIm1+ in Budding yeast 1

Escherichia coli

Eukaryotic initiation factor 4A

European Synchrotron Radiation Facility

Fibronectin-like

G-patch nucleolar protein 1

Glycine-rich patch

Guanosine triphosphate

helix-bundle

Hepatitis C virus

ATP-dependent DNA helicase Hel308

4-(2-hydroxyethyl)-1-piperazineethanesulfonic acid

Homo sapiens

Homo sapiens

Human Sap Homolog 155

Intron-lariat spliceosome

Internal stem-loop 


\section{Abbreviations}

Isy1

ITC

K

$K_{\mathrm{cat}}$

$K_{\mathrm{M}}$

l

LSm

$\mathrm{m}_{3} \mathrm{G}$

$m^{7} G$

$\min$

MPD

mRNA

NADH

MLE

Msl5

Mtr4

Mud2

NDP

NMR

NS3

NTC

N-terminal

NTP

NTR

Ntr1
Interactor of SYf1p 1

Isothermal titration calorimetry

Kelvin

Turnover numbered

Michaelis constant

Liter

U6 snRNA-associated Sm-like protein

2,2,7-trimethyl-guanosine

7-methylguanosine

Minute

2-Methyl-2,4-pentanediol

messenger RNA

Nicotinamide adenine dinucleotide

maleless

Mud Synthetic-Lethal 5

mRNA transport regulator MTR4

Mutant U1 Die 2

Nucleoside diphosphate

Nuclear magnetic resonance

Non-structural protein 3

NineTeen complex

Amino-terminal

Nucleoside triphosphate

NineTeen-Related

Nineteen complex-related protein 1 
Abbreviations

\begin{tabular}{|c|c|}
\hline $\mathrm{OB}$ & Oligonucleotide-binding \\
\hline OD & Optical density \\
\hline $\mathrm{OH}$ & hydroxy group \\
\hline PAGE & Polyacrylamide gel electrophoresis \\
\hline PcrA & plasmid copy reduced A \\
\hline PDB & Protein Data Bank \\
\hline PEG & Polyethylene glycol \\
\hline PEP & 2-phosphoenolpyruvate \\
\hline PETRA III & Positron-Elektron-Tandem-Ring-Anlage III \\
\hline Pfa1 & Prp forty-three associated 1 \\
\hline Poly-A & Poly-adenine \\
\hline Poly-U & Poly-uridine \\
\hline Pif1 & Petite Integration Frequency 1 \\
\hline pre-mRNA & precursor mRNA \\
\hline Prp & pre-mRNA-processing factor \\
\hline Rad3 & RADiation sensitive 3 \\
\hline RES & pre-mRNA retention and splicing complex \\
\hline RHA & RNA helicase A \\
\hline RIG-I-like & Retinoic acid inducible gene I \\
\hline r.m.s.d. & Root-mean-square deviation \\
\hline RNA & Ribonucleic acid \\
\hline RNP & Ribonucleoprotein \\
\hline rpm & Revolutions per minute \\
\hline sc & Saccharomyces cerevisiae \\
\hline S. cerevisiae & Saccharomyces cerevisiae \\
\hline
\end{tabular}




\section{Abbreviations}

S. pombe

SF

SF

Ski2

Slu

Sm

Snu

snRNP

snRNA

$\mathrm{S}_{\mathrm{N}}$

Spp

Ss

Sub2

Tris

tRNA

$\mathrm{U}$

Upf1

U-snRNP

WH

Yju2
Schizosaccharomyces pombe

superfamily

splicing factor

Superkiller protein 2

Synthetic lethal with U2 snRNA protein

Small nuclear ribonucleoprotein

small nuclear ribonucleic associated

small nuclear RNP

small nuclear RNA

Nucleophilic substitution mechanism

Suppressor of PRP protien

single-stranded

Suppressor of BRR1 protein 2

2-Amino-2-(hydroxymethyl)propane-1,3-diol

transfer RNA

Uracil

Up-frameshift suppressor 1

uridine-rich small nuclear ribonucleic particle

winged-helix

Protein CWC16 


\section{Acknowledgments}

First and foremost, I would like to express my gratitude to Prof. Dr. Ralf Ficner for giving me the opportunity to work on this exciting project. His competent and kind supervision over the past years has always given me great guidance and I appreciate his flexibility in giving me the chance to develop and execute own ideas.

I am also grateful to Prof. Dr. Reinhard Lührmann and Prof. Dr. Holger Stark for their participation in my Thesis Advisory Committee and providing useful input during the thesis committee meetings.

Furthermore, I would like to thank Dr. Sarah Adio, Prof. Dr. Kai Tittmann and Prof. Dr. Markus Bohnsack for willingly accept to be part of my extended examination board.

I am additionally very grateful to all present and former members of the Department of Molecular Structural Biology for making every working day an enjoyable day. Due to the great working atmosphere in the department even the most frustrating days turned out to be manageable. In particular, I want to thank Dr. Achim Dickmanns for his constant support, which started already early on during my bachelor thesis. He does an outstanding job in the organization of the lab and is always willing to give any kind of advice. A very special thanks goes to Dr. Piotr Neumann, who very patiently guided me through all crystallographic concerns. His knowledge and ideas were key in finding solutions for many problems and it had a great impact on the quality of my work. But I did not only learn a lot from him, I also deeply enjoyed all the nonscientific discussions we had and the hours I have spend in his office talking about all sorts of things. I am also grateful to Dr. Thomas Monecke for sharing his opinion with me on many topics and being a very joyful office mate. I thank Marita Kalck and Susanne van Beckum for their patience answering thousands of bureaucratic questions I had. I am grateful for the valuable contribution of my bachelor student Melina Fonfara and the outstanding assistance in the lab of Kelly Libnik. A very big thank you goes to all other fellow colleagues: Sven, Johannes, Marcel, Kathi, Jana, Marieke, Alaa and Tim. You all did not only make the lab a fun place to work, but I also really enjoyed the countless evenings together which were mostly accompanied by some beers and funny conversations.

Last but not least, I am extremely grateful to my parents and deceased grandparents for their constant and unconditional support. Sarah and all of my friends have been of great importance to me during the past four years. Thank you for being there for me! 